\title{
Novel causes, mechanisms and therapeutic strategies in mitochondrial disease
}

Citation for published version (APA):

Theunissen, T. (2018). Novel causes, mechanisms and therapeutic strategies in mitochondrial disease. [Doctoral Thesis, Maastricht University]. Gildeprint Drukkerijen. https://doi.org/10.26481/dis.20180418tt

Document status and date:

Published: 01/01/2018

DOI:

10.26481/dis.20180418tt

Document Version:

Publisher's PDF, also known as Version of record

\section{Please check the document version of this publication:}

- A submitted manuscript is the version of the article upon submission and before peer-review. There can be important differences between the submitted version and the official published version of record.

People interested in the research are advised to contact the author for the final version of the publication, or visit the DOI to the publisher's website.

- The final author version and the galley proof are versions of the publication after peer review.

- The final published version features the final layout of the paper including the volume, issue and page numbers.

Link to publication

\footnotetext{
General rights rights.

- You may freely distribute the URL identifying the publication in the public portal. please follow below link for the End User Agreement:

www.umlib.nl/taverne-license

Take down policy

If you believe that this document breaches copyright please contact us at:

repository@maastrichtuniversity.nl

providing details and we will investigate your claim.
}

Copyright and moral rights for the publications made accessible in the public portal are retained by the authors and/or other copyright owners and it is a condition of accessing publications that users recognise and abide by the legal requirements associated with these

- Users may download and print one copy of any publication from the public portal for the purpose of private study or research.

- You may not further distribute the material or use it for any profit-making activity or commercial gain

If the publication is distributed under the terms of Article $25 \mathrm{fa}$ of the Dutch Copyright Act, indicated by the "Taverne" license above, 
Novel causes, mechanisms and therapeutic strategies in mitochondrial disease 
(C) Tom E. J. Theunissen, Maastricht 2018

\section{ISBN:}

978-94-6233-923-1

Cover Design:

Ruby Zelissen

Layout and Printed by: Gildeprint

Published by:

Tom Theunissen 


\section{Novel causes, mechanisms and therapeutic strategies in mitochondrial disease}

\section{Proefschrift}

ter verkrijging van de graad van doctor aan de Universiteit Maastricht, op gezag van de Rector Magnificus, Prof. dr. Rianne M. Letschert volgens het besluit van het College van

Decanen in het openbaar te verdedigen op woensdag 18 april 2018 om 14:00 uur.

door

Tom Elisabeth Joseph Theunissen

geboren te Bilzen op 31 mei 1989 


\section{Promotor:}

Prof. dr. H.J.M. Smeets

\section{Co-promotores:}

Dr. I.F.M. de Coo (Erasmus MC)

Dr. R. Szklarczyk

\section{Beoordelingscommissie:}

Prof. dr. P.M. Steijlen (voorzitter)

Prof. dr. J.C.S. Kleinjans

Dr. R.J.T. Rodenburg (Radboud UMC)

Dr. R.H. Houtkooper (AMC)

The research described in this thesis was funded by the Prinses Beatrix Spierfonds and stichting Metakids, which is gratefully acknowledged by the author.

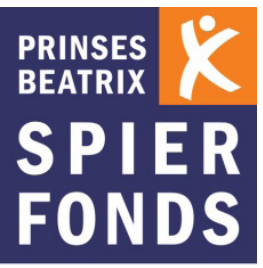

KRACHTIG TEGEN SPIERZIEKTEN

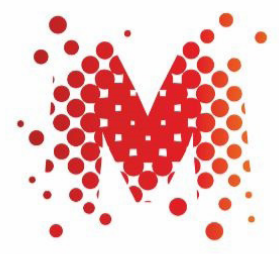

METAKIDS 


\section{Contents}

$\begin{array}{lll}\text { Chapter } 1 & \text { General Introduction and Thesis Outline } & 7\end{array}$

Chapter 2 Whole exome sequencing is the preferred, first strategy to identify known and novel causes of mitochondrial disease

Chapter 3 Rapid resolution of blended or composite multigenic disease in infants by whole exome sequencing

Chapter $4 \quad$ MRI abnormalities reveal severe Perrault syndrome due to CLPP defects

Chapter 5 Selection and characterization of palmitic acid responsive patients with an OXPHOS complex I defect

Chapter 6 An ERBB2 mutation as a potential, novel cause of Hirschprung's disease

Chapter 7 Differences in strength and timing of the mtDNA bottleneck between zebrafish germline and non-germline cells

Chapter 8 General Discussion

Summary

Samenvatting 



\section{CHAPTER 1}

General Introduction and Thesis Outline 
Chapter 1 


\section{Introduction}

Mitochondrial diseases are the most common inheritable metabolic diseases, affecting approximately 1 in 5000 individuals. They result from an insufficient capacity to produce adenosine triphosphate (ATP), caused by abnormalities in the oxidative phosphorylation (OXPHOS) system (1). As a result, a broad variety of, especially, high energy demanding organs systems can be affected, often leading to multi-systemic disease with brain, muscle and nerve symptoms involved. As illustrated by MELAS (Mitochondrial Encephalomyopathy, Lactic Acidosis, and Stroke-like episodes) and MIDD syndrome (Maternally inherited diabetes and deafness), which can both arise from the same m.3243A>G mutation in the mtDNA, mitochondrial genetic defects can be pleiotropic in their clinical manifestation (2). Besides the clinical heterogeneity, mitochondrial diseases are also highly heterogeneous in their genetic origin, where similar disease phenotypes can result from different gene defects. For example, Leigh syndrome can be caused by defects in more than 60 different genes. As the OXPHOS system is under a dual genetic control, mitochondrial diseases can be caused by both, mitochondrial DNA (mtDNA) and/or nuclear gene defects (3). This clinical and genetic heterogeneity complicates the identification of the genetic basis of mitochondrial disease, where a substantial part of the nuclear genes and defects still remain to be discovered. As, among others, illustrated in "A neurological perspective on mitochondrial disease" by Robert McFarland (4), the genetic diagnosis of mitochondrial disorders traditionally relies on numerous clinical and biochemical investigations, where extensive evaluation of clinical symptoms, mitochondrial biochemistry, histology/histochemistry and mtDNA quality and quantity are performed to select candidate genes for targeted sequencing analysis. Such candidate genes are especially based on previously characterized gene-phenotype associations, where one has attempted to relate single, or groups of gene defects to specific combinations of clinical and biochemical features. However, due to the tremendous clinical and biochemical heterogeneity in mitochondrial diseases, with significant phenotypic overlap involved, unique gene-phenotype relations most often do not exist, complicating the use of targeted sequencing approaches. As a result, many patients with mitochondrial disease remain undiagnosed. The development of next generation sequencing (NGS) has the potential to revolutionize the identification of the genetic causes in mitochondrial disease, as it allows identification of all genetic variations in a patient, including those in novel genes, and it can be applied as a single method to evaluate both the mtDNA and exome or genome. Considering all genetic variants is crucial, not only to establish a correct genetic diagnosis in the patient and prevent disease transmission towards offspring, by applying prenatal diagnosis (PND) or preimplantation genetic diagnosis (PGD), but also to improve our understanding of the pathophysiological basis of mitochondrial disease, with the ultimate aim to develop effective treatment options. Currently no curative treatment 
options are available for mitochondrial diseases as a whole, although for some specific mitochondrial defects, effective, targeted, therapies exist.

\section{The oxidative phosphorylation system (OXPHOS) and OXPHOS deficiencies}

The mitochondrial oxidative phosphorylation system, present in almost all cells of the body, produces ATP in a highly efficient manner. It depends on an electron transport chain (ETC) that consists of four protein complexes and an ATP synthase (complex V), together making up the OXPHOS system. The ETC substrates NADH and FADH ${ }_{2}$, which are generated during glycolysis, the citric acid cycle and beta-oxidation of fatty acids, donate their electrons directly to complex I and complex II of the ETC, respectively. Subsequently, electrons flow through the four ETC complexes and the electron carriers ubiquinone (Coenzyme Q10) and Cytochrome C, where complex I, II, III and IV are proton pumps, responsible for establishing a proton gradient $\left(\mathrm{H}^{+}\right)$across the mitochondrial inner-membrane (Figure 1 ). As a consequence, proton reflux occurs through complex $\mathrm{V}$, an ATP synthase that converts $\mathrm{ADP}+\mathrm{Pi}$ tot ATP. During this process oxygen $\left(\mathrm{O}_{2}\right)$ is reduced by complex IV, leading to the production of $2 \mathrm{H}_{2} \mathrm{O}$ molecules $(5,6)$. In addition, there is a continuous generation of reactive oxygen species (ROS) during the OXPHOS due to leakage of electrons from the complexes, with complex I and III being the main source of electron leakage. Other important proteins involved in electron leakage include P66shc, MAO, and the UCPs (7). Although, ROS have an important role in cell signaling, increased ROS can have highly damaging and mutagenic effects when levels become too high. ROS affects mitochondrial function in vicious circular way, as increased ROS levels are not only a consequence of defects in the OXPHOS system, but can also be a cause of OXPHOS dysfunction by introducing mutations within the mtDNA (8). Isolated or combined OXPHOS deficiencies, which are typically involved in primary mitochondrial disease, can be due to genetic defects in the core-subunits that directly make up the OXPHOS system, and in the electron carriers and cofactors needed for proper ETC functioning. The genes encoding the subunits of the OXPHOS protein-complexes are located in both the mtDNA and nDNA, except for complex II that is fully nDNA encoded. Alternatively, OXPHOS deficiencies and mitochondrial disease can be caused by any of the hundred nDNA encoded proteins that function in mitochondrial OXPHOS complex assembly (assembly factors), mtDNA replication and maintenance, mtDNA transcription and translation, and other proteins that are more indirectly related to OXPHOS function. 


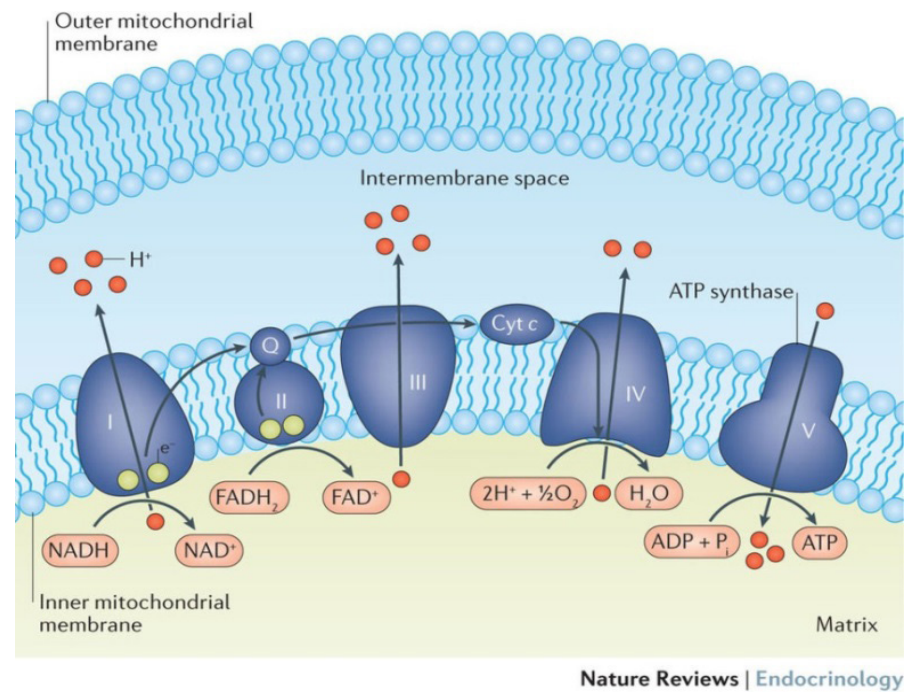

Figure 1: The OXPHOS system. $\mathrm{NADH}$ and $\mathrm{FADH} \mathrm{H}_{2}$ donate electrons to respectively complex I and complex II of the electron transport chain (ETC), resulting in an electron flow through the five mitochondrial ETC complexes and two electron carriers (ubiquinone and cytochrome C). Consequentially, complex I, III and IV are able to transport $\mathrm{H}^{+}$protons across the mitochondrial inner-membrane from the matrix to the inter-membrane space, resulting in a proton gradient. The protons flow back to the matrix through complex $V$, which is the ATP-synthase, capable to generate ATP. Adapted from Jasmine Chow et al., Nature Reviews, 2016.

\section{mtDNA, mtDNA disease and mtDNA inheritance}

Mitochondria contain their own DNA, which consists of a 16,569 base pair circular double stranded molecule, containing 37 genes (Figure 2). 22 genes encode tRNAs required for the translation of mitochondrial encoded proteins, 2 encode the $12 \mathrm{~S}$ and $16 \mathrm{~S}$ rRNAs, which form part of the mitochondrial ribosomes, and 13 encode core subunits of the mitochondrial OXPHOS complexes I, III, IV, and V. In contrast to the nuclear genome, the mtDNA contains no introns and harbors a displacement loop (D-loop) with the transcriptional promoters and origin of replication. As a result of this specific genetic makeup, the mitochondria have their own system for replication, transcription and translation of the mtDNA. With exception of the above mentioned tRNAs and rRNAs, all other components required for these processes are encoded by the nDNA (9). The mtDNA is a multi-copy genome that is strictly maternally inherited, as the paternal mtDNA is degraded when it enters the oocyte during fertilization (10). The mtDNA copy-number highly differs among cell-types and tissues, ranging from only a few copies to more than 100.000 copies in the oocyte. The mtDNA copy number and number of mitochondria is mainly dependent on the energy demands of the cell or tissue involved. As each mitochondrion, and thus each individual cell contains many copies of mtDNA, in case of an mtDNA mutation, a mixture of wild-type and mutant mtDNA molecules 
can coexist in different ratio's, which is known as heteroplasmy (11). Heteroplasmy levels can differ between cells, tissues and significantly change over time. A state where all mtDNA molecules are identical is referred to as homoplasmy.

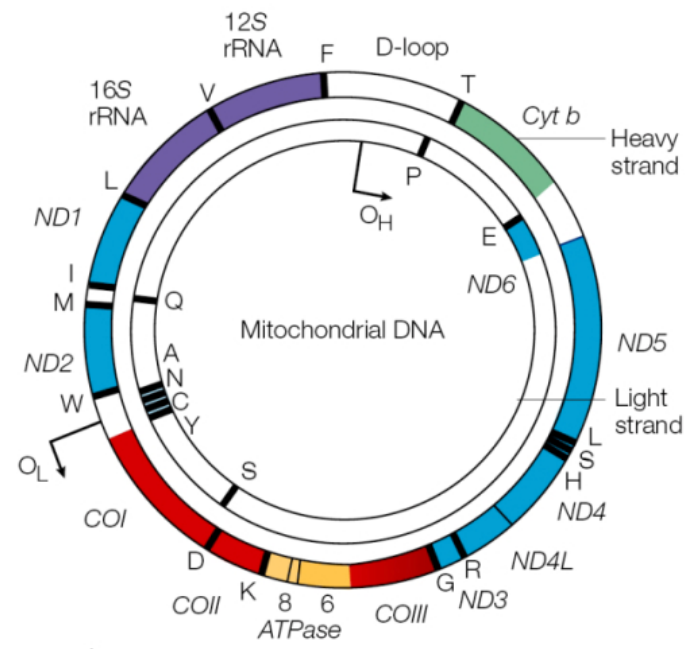

Figure 2: The mtDNA. The human circular mitochondrial DNA consists of $16.569 \mathrm{bp}$, encoding 22 tRNA's, 2 rRNA's, and 13 core-subunits of the OXPHOS complexes. Complex I genes are Blue; complex III genes are green; complex IV genes are red; complex V genes are orange; rRNA genes are purple; tRNA genes are black. Adapted from Heidi Chial et al., Nature Education, 2008.

Genetic diagnosis of mtDNA-diseases requires screening of all $37 \mathrm{mtDNA}$ genes for large scale rearrangements and point mutations, determining heteroplasmy levels and characterizing whether these occur familial or de novo. Also, multiple mtDNA deletions or a reduction in mtDNA copy number can be found, which are either caused by a defect in a nuclear gene, involved in mtDNA replication, or by environmental factors (medication, aging) (12). The mutated mtDNA copy-number level needs to exceed a threshold before a biochemical OXPHOS defect will occur, leading to mitochondrial disease symptoms. This threshold is dependent on the mutation, cell-type and energy demands, where disease manifestations can occur at low heteroplasmy levels (10\%-30\% in MIDD due to the m.3243A>G mutation) (2) to relatively high heteroplasmy levels (>70-90\% in NARP/Leigh syndrome due to m.8993T>C/G mutation) (13). mtDNA defects can be clinically heterogeneous, as, the same mtDNA mutation can lead to different symptom manifestations at varying heteroplasmy levels, as illustrated by the m.8993T>G mutation in the ATP6 gene causing the NARP phenotype in individuals with moderate levels ( 70\%-90\%) of the pathogenic variant, and Leigh-syndrome in patients with more than $90 \%$ mutated mtDNA $(13,14)$. Patients with lower than $70-75 \%$ heteroplasmy remain asymptomatic. For the m.3243A>G mutation, even more extensive 
heterogeneity exists in the tissues being affected (brain, muscle, heart, kidney) and the severity of the clinical manifestations, which can only partly be explained by the mutation load. Only, Leber Hereditary Optic Neuropathy (LHON) has a strong genotype-phenotype correlation with $>95 \%$ of the cases explained by 3 generally homoplasmic mtDNA mutations. Table 1 shows some of the frequent mtDNA mutations and the related clinical phenotypes (15). Although highly dependent on the mutation heteroplasmy levels and tissues involved, defects in the mtDNA encoded tRNAs often cause mitochondrial syndromes such as MIDD (maternally inherited diabetes and deafness), MELAS (mitochondrial encephalomyopathy, lactic acidosis, and stroke-like episodes), MERRF (myoclonic epilepsy with ragged red fibers) and, as part of large scale deletions, PEO (progressive external ophthalmoplegia) and KSS (Kearns-Sayre syndrome) (16). With respect to the mt-rRNAs, there is only evidence for a role in mtDNA disease for the m.1555A>G mutation in the 12S ribosomal RNA (RNR1) (17).

Rapid changes in mtDNA heteroplasmy, causing variation in mutation load between generations, have been explained by a phenomenon that is known as the mtDNA bottleneck. The mtDNA bottleneck occurs during early embryogenesis in both germ cells and in somatic cells, where a decrease in the number of mitochondrial genomes repopulating the offspring of the next generation causes a sampling effect during transmission. Cell division without mtDNA replication and random segregation of the mtDNA molecules yield a small founder population in the primordial germ cells, which is being amplified to large numbers during oogenesis. In addition to genetic drift, evidence for a role for active selection mechanisms in the transmission of mtDNA has been identified as well. This unpredictable transmission of the mtDNA mutation load makes it difficult to estimate mtDNA disease recurrence risks in individual cases. Also, for familial mutations, prenatal diagnosis is not reliable, because of the difficulty to accurately predict the phenotype from the mutation load in chorionic villus or amniotic fluid cells (18). Pre-implantation genetic diagnosis (PGD) to select healthy embryos is a better alternative (19). We calculated for all mutations that a 95\% or higher chance of being unaffected correlated with a muscle mutation level of $18 \%$ or less for all mutations (20). This threshold is being used in clinical practice to prevent transmission of mtDNA diseases via selection of, most likely, unaffected embryos (blastomeres) with mutation loads below this "safe" threshold. 


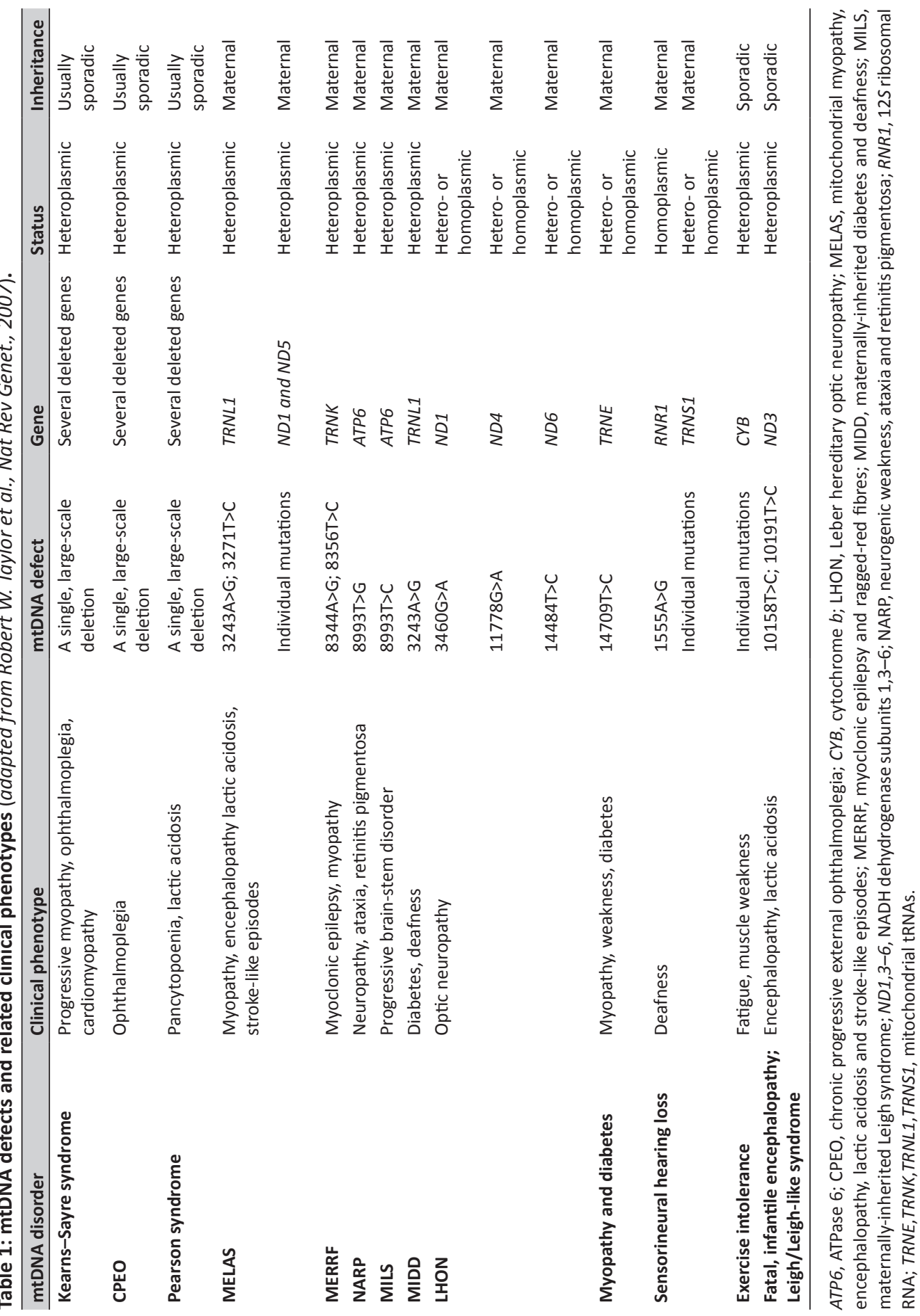


The low mtDNA copy-number at the bottom of the bottleneck also increases the risk for de novo mutations to reach significant heteroplasmy levels with functional consequences (2123). It has been proposed that the relative high mtDNA mutation rates result from mtDNA replication errors, the localization of the mIDNA near the OXPHOS system, which is the main site of ROS production, and its lack of protective histones, but conflicting data exists (16). Also, environmental, toxicological, factors may cause de novo mutations and/or influence mtDNA inheritance $(16,24)$. De novo mtDNA mutations were reported to occur in $\sim 25 \%$ of the patients with mtDNA mutations. The recurrence risk, for both large scale deletions and point-mutations, is very low, and prenatal diagnosis can be offered for reassurance (25).

\section{nDNA defects}

Mitochondrial diseases caused by nDNA defects may inherit in an autosomal or X-linked recessive fashion, covering homozygous, compound heterozygous and hemizygous mutations, or in an autosomal dominant fashion. In addition, germ-line de novo or mosaic mutations may be involved. The estimated number of nuclear genes with a mitochondrial function is around $1500(26,27)$, of which $>250$ genes have been shown to be involved in mitochondrial disease (28). Yet, many of the nDNA encoded defects that underlie OXPHOS deficiency remain to be discovered. Combined or isolated OXPHOS deficiencies may arise from defects in the genes that encode the core-subunits, assembly factors, electron carriers and cofactors that are directly needed for proper OXPHOS function (Figure 3). The clinical consequences of defects in these OXPHOS genes are highly heterogeneous with significant overlap involved, complicating the establishment of a genetic diagnosis based on predefined panels of candidate genes. The latter is illustrated by Leigh syndrome, which can, besides from having a mtDNA origin, result from defects in many of the different complex I subunits and assembly factors, but also complex II subunits and assembly factors, complex III subunits, complex IV subunits and assembly factors, biosynthesis of CoQ10, and other nDNA genes that are more indirectly related to OXPHOS function (29-32). Only in combination with an isolated complex IV deficiency, the genetic origin of Leigh syndrome is in most cases found in the SURF-1 gene (33). As indicated in Figure 3, most genetic defects have been identified in the subunits and assembly factors of complex I, which is also the largest component of the respiratory chain, in which OXPHOS deficiencies are, with $>30 \%$, the most prevalent (34-37). 


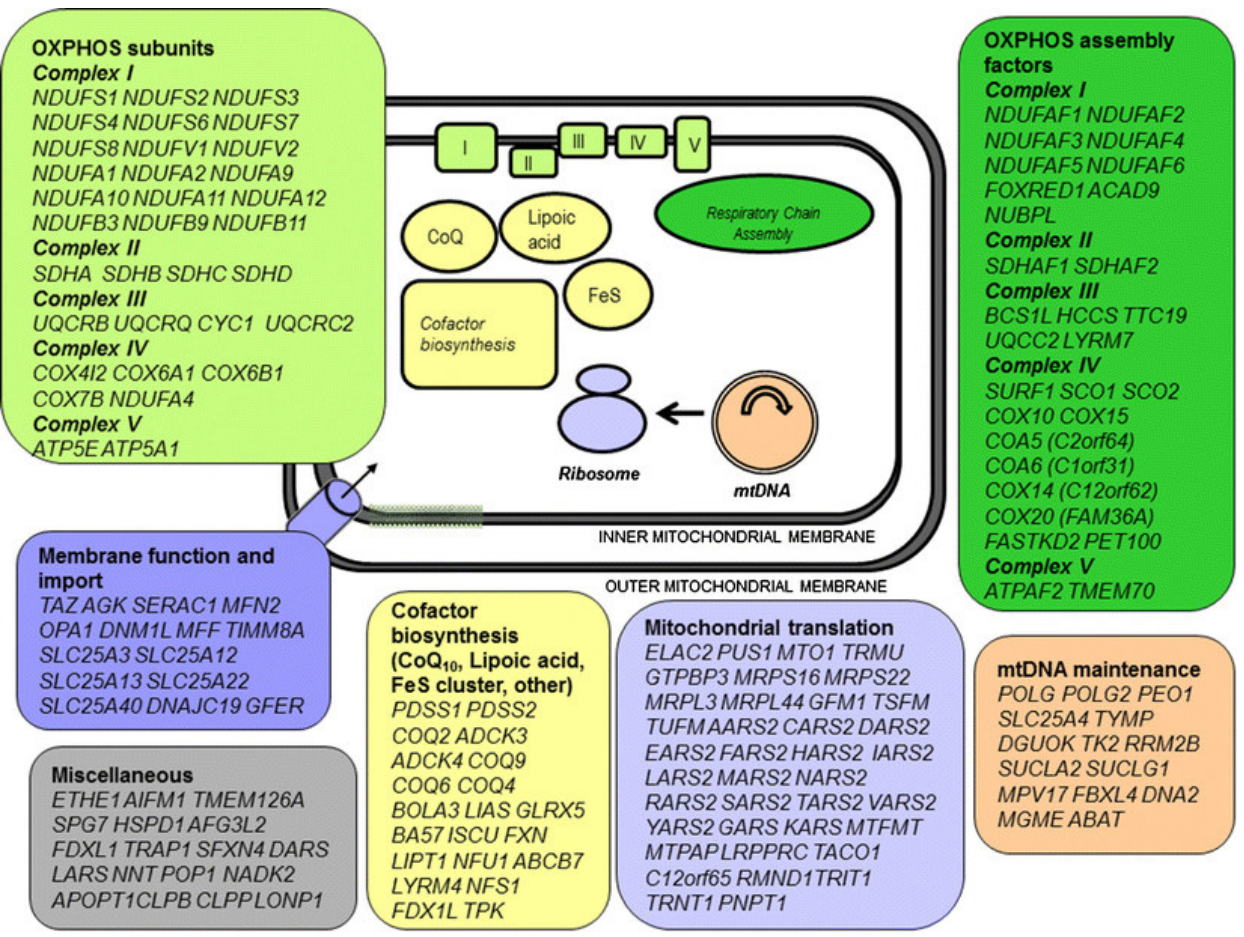

Figure 3: Nuclear genes related to mitochondrial disease, grouped according to their function in mitochondrial metabolism. Defects identified in the OXPHOS complex subunits (light green) and OXPHOS assembly factors (dark green), where complex I defects, and resulting complex I deficiencies, are the most common respiratory chain defect. Also defects in the OXPHOS co-factors, co-factor biosynthesis pathways (yellow), mtDNA replication/maintenance processes (peach), transcription, and translation (light blue) can cause isolated or combined OXPHOS deficiency. More indirectly, defect in proteins that function in membrane integrity and import of substrates (dark blue) or other processes (grey) such as mitochondrial protein metabolism, fusion and fission may cause OXPHOS deficiency. Adapted from Rahman S, J Inherit Metab Dis, 2015.

Defects in the enzymes that function in mtDNA replication and maintenance (Figure $3)$, which are all nDNA encoded and imported into the mitochondria, can also result in OXPHOS deficiencies by causing a decrease in mtDNA copy number (depletion) and/or an accumulation of point-mutations and deletions in the mtDNA (38). Crucial factors in the mitochondrial replisome are the mtDNA polymerase- $\gamma$ (POLG, POLG2), the mtDNA helicase Twinkle (c10orf2), and the single stranded DNA binding protein mtSSB (39) (Figure 4). Mutations in polymerase- $\gamma$ typically cause mtDNA depletion, large scale mtDNA deletions, or accumulation of point-mutations. Polymerase- $\nu$ defects are heterogeneous in their clinical manifestation and age of onset, where almost any organ system can be involved, and broad and overlapping phenotypes can be distinct clinical entities or consist of a spectrum of overlapping phenotypes. Examples of POLG related syndromes are the 
autosomal recessive or dominant progressive external ophthalmoplegia (ar/adPEO), AlpersHuttenlocher syndrome (AHS), childhood myocerebrohepatopathy spectrum (MCHS), myoclonic epilepsy myopathy sensory ataxia (MEMSA), and the ataxia neuropathy spectrum (ANS). The latter covering mitochondrial recessive ataxia syndrome (MIRAS) and sensory ataxia neuropathy dysarthria and ophthalmoplegia (SANDO) $(40,41)$. Dominant POLG mutations can also lead to premature ovarian failure (POF). Another cause of large scale mtDNA deletions and/or depletion syndromes are defects in the mtDNA helicase Twinkle (Table 2), which can, among others, cause adPEO and encephalopathy $(41,42)$. Not directly interacting with the replisome, but crucial for mtDNA maintenance, are the enzymes related to the mitochondrial salvage pathway, responsible for the availability of the nucleosides and nucleotides for mtDNA replication and transcription. This process also covers enzymes involved in cytoplasmic nucleotide metabolism and mitochondrial membrane transport, as these influence the substrate availability for the mitochondrial salvage pathway (Figure 4). Defects in genes related to mitochondrial nucleotide metabolism, such as TYMP (TP), TK2, DGUOK, RRM2B, SUCLA1, SUCLA2, ANT1 and MPV17 can cause instability of the mtDNA (Table 2) and consequentially cause a broad variety of mtDNA depletion disorders, often classified as myopathic, encephalomyopathic, hepatocerebral, neurogastrointestinal, or mtDNA deletion disorders, such as progressive external ophthalmoplegia (PEO) or mitochondrial neurogastrointestinal encephalomyopathy (MNGIE) $(38,43)$. Yet, mtDNA deletions may also arise from defects in genes that are related to mitochondrial network dynamics, such as OPA1, or external environmental factors.

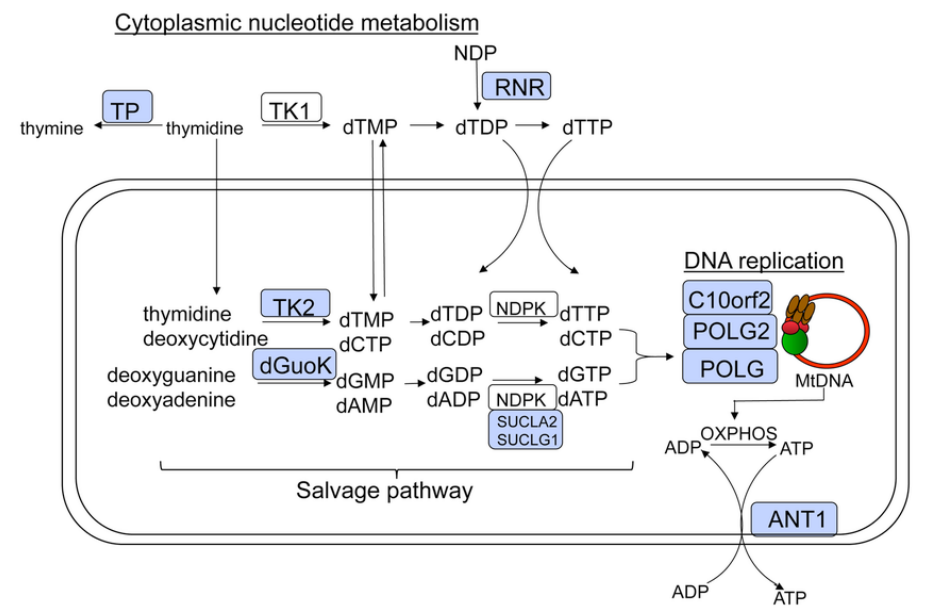

Figure 4: genes involved in mtDNA replication. Indicated the enzymes directly interacting in the replisome, such as POLG, POLG2, Twinkle (c10orf2) and enzymes related to the mitochondrial salvage pathway, such as TP, TK2, DGUOK, SUCLA2, ANT1, required for maintaining the mitochondrial nucleotide pool. Gene functions are described in Table 2. Adapted from Copeland WC, Crit Rev Biochem Mol Biol, 2012. 
Table 2: mtDNA replication genes in which defects have a consequence on mtDNA.

\begin{tabular}{lll}
\hline gene & mtDNA consequence & function \\
\hline POLG1, POLG2 & depletion, deletions & Polymerase, Polymerase accessory subunit \\
Twinkle (c10orf2) & depletion, deletions & mtDNA helicase \\
TP (TYMP) & depletion, deletions & thymidine phosphorylase \\
DGUOK & depletion & mitochondrial deoxyguanosine kinase \\
TK2 & depletion & mitochondrial thymidine kinase \\
RRM2B & depletion & subunit of ribonucleotide reductase \\
ANT1 (SLC25A4) & deletions & adenine nucleotide translocator \\
MPV17 & depletion & mitochondrial inner membrane protein \\
SUCLA1 & depletion & subunit succinate-coA ligase \\
SUCLA2 & depletion & subunit succinate-coA ligase \\
SUCLG1 & depletion & subunit succinate-coA ligase \\
\hline
\end{tabular}

Except for the mtDNA encoded tRNAs and rRNAs, the machinery required for mitochondrial protein synthesis and homeostasis, including the transcriptional and translational regulators, is encoded in the nDNA (44). OXPHOS deficiency resulting from mtDNA or nDNA defects that affect the processes of mitochondrial transcription, protein translation and degradation, often lead to combined complex deficiencies (45). Whereas the transcriptional machinery covers a limited number of components, including the transcriptional activator TFAM, RNA polymerase POLRMT, transcription factor TFB2M, transcript termination factor MTERF and also the above described genes involved in nucleotide metabolism, the components of the translational machinery are much more extensive (Figure 3)(46). Besides the mtDNA encoded tRNAs and rRNAs, the translational machinery consists of many nDNA encoded proteins that are needed for ribosome assembly and function, covering amino-acyl tRNA synthetases, tRNA modifying enzymes, translation initiation factors, elongation factors, termination factors and release factors (9). nDNA encoded defects that interfere with mitochondrial translation have been described to cause a broad variety of symptoms, including encephalopathy, cardiomyopathy, Leigh Syndrome and liver failure (47-52).

Other, more indirect, causes of OXPHOS deficiency can be found in the processes of fusion and fission, phospholipid/membrane function, and substrate transport. The mitochondrial network is highly dynamic and regulated by many factors that function in the process of fusion and fission, where, in healthy condition, a balance between both processes exist to maintain mitochondrial network integrity and OXPHOS capacity. Fusion of the outer mitochondrial membranes is especially performed by the mitofusin family, with a key role for mitofusin 1 (MFN1) and mitofusin 2 (MFN2), whereas inner-membrane fusion is mediated by OPA1. Fission is mediated by FIS1, which is anchored to the outer-membrane and is a component of the mitochondrial ARCosome complex, and the dynamin related protein DRP1 $(53,54)$. Defects in MFN2 are the most common cause of Charcot-Marie-Tooth disease and have been related to motor and sensory neuropathies. Defects in OPA1 have 
especially been associated with autosomal dominant optic atrophy (ADOA) and chronic progressive external ophthalmoplegia (CPEO). Yet, a variety of phenotypes has been related to fusion and fission defects (55). Finally, mitochondrial dysfunction can also be a secondary defect in genetic syndromes or neuromuscular disease (56). As a result, a broad variety of clinical entities, with or without OXPHOS dysfunction involved, may be underlain by a mitochondrial gene defect. Obviously, this complicates the identification of the underlying gene defect using targeted sequencing approaches.

\section{Next-generation sequencing (NGS)}

Next-generation sequencing (NGS), also called Massive Parallel Sequencing, is a recently developed technique to screen a large number of sequences in parallel, allowing the analysis of all genes at the same time. This enables whole-genome sequencing or wholeexome sequencing of an individual patient and can therefore contribute considerably to the characterization of known and novel nDNA defects that underlie mitochondrial disease. In massive parallel sequencing, using Illumina technology platforms, DNA bridgeamplification, which is needed to obtain sufficient signal for acquisition, occurs when the DNA library fragments are hybridized to a flow-cell, creating individual PCR clusters that derive from an single biological DNA fragment (57). Clusters are sequenced (paired-end) in parallel using universal adaptors and primers, fluorescent reversible terminator nucleotides, and a polymerase. Each sequenced (read)-fragment can be traced back to its originating biological template fragment. NGS applications have a high coverage and allow deepsequencing in for example tumor-tissue, but also have the capacity to detect rare clonal types, microbes, and mtDNA heteroplasmy levels, comprising as little as $1 \%$ of the original sample (58). The ability to calculate wild-type versus mutant molecule ratios is crucial in many quantitative applications, for example for accurately defining mtDNA heteroplasmy levels, homo- or heterozygosity of a nDNA variant, DNA copy-number variations, but also for characterizing somatic mutations or mosaicisms. Besides, more recent applications in the field of transcriptomics (RNA-seq) rely on this quantitative application (59-61). NGS is faster and has a higher sequencing capacity than Sanger sequencing, as it allows the pooling of different samples on a single flow-cell lane using index-sequence labels. Yet, compared to Sanger sequencing ( $800 \mathrm{bp}$ ), read-lengths can be relative short, which can become critical during the mapping process, especially when genomic regions consist of highly repetitive or polymorphic regions. Read lengths highly differ among the different NGS vendors, where Illumina technology (MiSeq, NextSeq, Hiseq platforms) allows read-lengths of only 36$300 \mathrm{bp}$, the PacBio system can generate read-lengths of more than $10 \mathrm{~kb}$, and complimentary use of both systems is advocated to get a complete picture. Anyhow, the main advantage of WES and WGS is the fact that all genetic variants in respectively the protein coding regions 
(exons) or complete genome, can be identified and evaluated for being disease causing. Massive parallel sequencing offers many advantages above traditional Sanger sequencing based analysis of targeted gene panels, which only cover a limited number of gene defects that are associated with the disease-phenotype. NGS is unbiased and identifies all variants at the same time and is much faster than the sequential analysis of candidate genes in Sanger sequencing. It is also cheaper and has the potential to identify novel genes. However, a drawback of the NGS data is the interpretation of the huge number of variants that are being identified, including many variants of unknown significance, which need functional validation based on in vitro or in vivo models to prove a role in disease.

\section{Aims and outline of the thesis}

Mitochondrial disorders result from a reduced energy producing capacity in the mitochondria due to deficiencies of the oxidative phosphorylation (OXPHOS) system. They are characterized by highly heterogeneous and tissue-specific clinical manifestations, typically involving tissues with high energy requirements. Symptoms that are frequently observed concern among others, muscle weakness, lactic acidosis, epilepsy, mitochondrial encephalopathy, ataxia and cardiomyopathy. Mitochondrial diseases affect approximately one in 5,000 births. As the OXPHOS system is under a dual genetic control, mutations in both the mitochondrial (mtDNA) and nuclear DNA can lead to mitochondrial disease. Mainly as a consequence of the large genetic heterogeneity (possibly $>1500$ genes involved) and clinical variability, for more than $75 \%$ of the patients with an OXPHOS defect, but without an mtDNA mutation, a nuclear genetic cause was unknown at the start of this project in 2011. Our central hypothesis was that for this group of patients, whole exome sequencing would enable us to identify the underlying genetic cause, characterize the pathophysiological mechanisms and unravel possible targets for therapeutic intervention. This test this hypothesis, we defined 3 specific aims:

1) To develop and implement whole exome sequencing and novel bioinformatics approaches for the identification of gene defects in patients with a likely or possible mitochondrial disease. Characterization of the genetic defects is not only important for diagnosis and prognosis, but, especially in case of novel genes, will improve our insight into the diverse pathophysiological mechanisms and clinical manifestations in mitochondrial disease.

2) To characterize the functional effect of mutations in known and novel genes in patient cell lines and zebrafish. We tested if zebrafish could be used as a model to study the transmission of the mtDNA and characterize the mtDNA bottleneck, which plays a profound role in the unpredictable transmission of mtDNA disease. 
3) To identify, based on the genes and pathways involved, novel therapeutic targets. So far, the identification of genetic defects in patients with mitochondrial disease has only provided in limited cases a direct clue for therapeutic intervention.

In chapter $\mathbf{2}$ we describe the results of whole exome sequencing in a patient cohort of 117 patients, in which a genetic cause was highly likely. Most of the cases were pediatric patients ( $<18$ years old), with either a high suspicion of a mitochondrial disorder or with a disease phenotype in which mitochondrial defects are part of the differential diagnosis. A mitochondrial cause was highly likely in part of the patients based on the presence of mitochondria-related disease symptoms (Nijmegen Clinical Criteria for Mitochondrial Disease) and/or involvement of OXPHOS complex deficiencies, whereas in other, differential diagnostic cases only a minority would be expected to be caused by a mitochondrial defect. The cohort was tested first for the presence of mtDNA mutations by next-generation sequencing, followed by screening for POLG mutations by Sanger sequencing. Unsolved patients were analysed by whole exome sequencing (WES). Variants were filtered by bioinformatics approaches to select genes with rare, potentially harmful variants, fitting the presumed genetic model (autosomal or X-linked recessive, autosomal dominant or de novo). This approach was combined with SNP-array data of patients to identify large chromosomal copy number variants and to reduce the number of candidate genes by homozygosity mapping (regions of homozygosity) in consanguineous families. With this strategy, we expected to identify the genetic cause in the majority of the patients, involving novel genes in part of these patients. To improve identification and characterization of novel candidate genes, we implemented new bioinformatics tools for prioritizing candidate genes based on co-expression data, and we performed functional and complementation assays. We provide an overview of the genetic causes in the entire cohort, covering both mtDNA and nuclear gene mutations, and propose an efficient diagnostic strategy, directly starting with whole exome sequencing. As heterogeneous clinical phenotypes may not always be caused by a single gene defect, especially in consanguineous family, we showed in chapter $\mathbf{3}$ the importance of following-up all variants identified by whole exome sequencing that could in anyway be related to the clinical symptoms. We described 2 cases, in which whole exome sequencing revealed multiple genetic causes with overlapping (blended) or separate (composite) clinical symptoms, illustrating the importance of an unbiased, comprehensive approach. In chapter 4 we identify the genetic defect in a patient with particular MRIabnormalities. These MRI patterns turned out to be specific for the underlying gene, allowing the identification of additional patients with a similar gene defect, based on MRI characteristics only. Defects identified in novel genes in single patients or single families require in vitro and/or in vivo models to prove that these are causal for the clinical phenotype observed and to characterize their pathophysiological role. In chapter $\mathbf{5}$ we describe a novel 
gene defect identified in the TMEM126B gene, an assembly factor of OXPHOS complex I, in a patient with a complex I deficiency in muscle and fibroblasts. Lentiviral transduction of fibroblasts of the patient demonstrated that the TMEM126B defect caused the complex I deficiency (complementation assay). As the patient was reported to improve following a high-fat diet, we tested this responsiveness in vitro, and based on these results we developed an assay to test the OXPHOS responsiveness of patient cells to specific compounds, and the capability to restore OXPHOS capacity. Finally, in chapter 6, we describe a homozygous $E R B B 2$ variant, identified in a patient with Hirschsprung disease. Although, ERBB2 is a strong candidate gene and the variant seems to have damaging properties, definite proof requires functional validation. In case no deficiency is measurable in fibroblasts of the patient, it is an alternative to test the genetic defect in a whole organism, like the zebrafish. The application of the zebrafish model for nuclear gene mutations has been well-established and this work is currently ongoing for the ERBB2 gene. To demonstrate that the zebrafish would also be a suitable model for the mtDNA and mtDNA-associated abnormalities, we tested the basic mechanism of mtDNA transmission and the mtDNA bottleneck (chapter 7 ) during oogenesis and embryonic development. If comparable with humans this would extend the use of the zebrafish model to also this aspect of mitochondrial and mtDNA disease. In chapter $\mathbf{8}$, we give an overview of the different functional models that can be used to study mitochondrial defects. We discuss the advantages and disadvantages of different in silico, in vitro and in vivo models to test variant pathogenicity or study mitochondrial disease genes, where the choice for a particular model is ultimately dependent on the research question, but should be as simple as possible. Furthermore, we give an overview of the treatments that are currently clinically applied or, as for most of the treatment strategies, are being investigated in clinical trials and preclinical studies. Although mitochondrial treatment options are still limited, and often only supportive, we show the variety of tailored and generic treatment approaches that are under development and discuss their potential in treating mitochondrial disease, eventually going towards personalized treatment strategies. 


\section{References}

1. Gorman GS, Schaefer AM, Ng Y, Gomez N, Blakely EL, Alston CL, et al. Prevalence of nuclear and mitochondrial DNA mutations related to adult mitochondrial disease. Ann Neurol. 2015;77(5):753-9.

2. de Laat P, Janssen MC, Alston CL, Taylor RW, Rodenburg RJ, Smeitink JA. Three families with 'de novo' m.3243A > G mutation. BBA clinical. 2016;6:19-24.

3. Chinnery PF. Mitochondrial Disorders Overview. In: Adam MP, Ardinger HH, Pagon RA, Wallace SE, Bean LJH, Mefford HC, et al., editors. GeneReviews(R). Seattle (WA): University of Washington, SeattleUniversity of Washington, Seattle. GeneReviews is a registered trademark of the University of Washington, Seattle. All rights reserved.; 1993.

4. McFarland R, Taylor RW, Turnbull DM. A neurological perspective on mitochondrial disease. Lancet Neurol. 2010;9(8):829-40.

5. van den Heuvel L, Smeitink J. The oxidative phosphorylation (OXPHOS) system: nuclear genes and human genetic diseases. BioEssays : news and reviews in molecular, cellular and developmental biology. 2001;23(6):518-25.

6. Chow J, Rahman J, Achermann JC, Dattani MT, Rahman S. Mitochondrial disease and endocrine dysfunction. Nature reviews Endocrinology. 2017;13(2):92-104.

7. Lenaz G, Genova ML. Structure and organization of mitochondrial respiratory complexes: a new understanding of an old subject. Antioxidants \& redox signaling. 2010;12(8):961-1008.

8. Shokolenko I, Venediktova N, Bochkareva A, Wilson GL, Alexeyev MF. Oxidative stress induces degradation of mitochondrial DNA. Nucleic Acids Res. 2009;37(8):2539-48.

9. Taanman JW. The mitochondrial genome: structure, transcription, translation and replication. Biochim Biophys Acta. 1999;1410(2):103-23.

10. Sutovsky P, Moreno RD, Ramalho-Santos J, Dominko T, Simerly C, Schatten G. Ubiquitin tag for sperm mitochondria. Nature. 1999;402(6760):371-2.

11. Wai T, Ao A, Zhang X, Cyr D, Dufort D, Shoubridge EA. The role of mitochondrial DNA copy number in mammalian fertility. Biology of reproduction. 2010;83(1):52-62.

12. Wong LJ. Diagnostic challenges of mitochondrial DNA disorders. Mitochondrion. 2007;7(12):45-52.

13. Thorburn DR, Rahman J, Rahman S. Mitochondrial DNA-Associated Leigh Syndrome and NARP. In: Adam MP, Ardinger HH, Pagon RA, Wallace SE, Bean LJH, Mefford HC, et al., editors. GeneReviews(R). Seattle (WA): University of Washington, Seattle University of Washington, Seattle. GeneReviews is a registered trademark of the University of Washington, Seattle. All rights reserved.; 1993.

14. Claeys KG, Abicht A, Hausler M, Kleinle S, Wiesmann M, Schulz JB, et al. Novel genetic and neuropathological insights in neurogenic muscle weakness, ataxia, and retinitis pigmentosa (NARP). Muscle Nerve. 2016;54(2):328-33.

15. Taylor RW, Turnbull DM. Mitochondrial DNA mutations in human disease. Nature reviews Genetics. 2005;6(5):389-402.

16. Tuppen HA, Blakely EL, Turnbull DM, Taylor RW. Mitochondrial DNA mutations and human disease. Biochim Biophys Acta. 2010;1797(2):113-28.

17. Bindu LH, Reddy PP. Genetics of aminoglycoside-induced and prelingual non-syndromic mitochondrial hearing impairment: a review. International journal of audiology. 2008;47(11):7027.

18. Mitalipov S, Amato P, Parry S, Falk MJ. Limitations of preimplantation genetic diagnosis for mitochondrial DNA diseases. Cell Rep. 2014;7(4):935-7. 
19. Sallevelt SC, Dreesen JC, Drusedau M, Spierts S, Coonen E, van Tienen FH, et al. Preimplantation genetic diagnosis in mitochondrial DNA disorders: challenge and success. J Med Genet. 2013;50(2):125-32.

20. Hellebrekers DM, Wolfe R, Hendrickx AT, de Coo IF, de Die CE, Geraedts JP, et al. PGD and heteroplasmic mitochondrial DNA point mutations: a systematic review estimating the chance of healthy offspring. Hum Reprod Update. 2012;18(4):341-9.

21. Cree LM, Samuels DC, Chinnery PF. The inheritance of pathogenic mitochondrial DNA mutations. Biochim Biophys Acta. 2009;1792(12):1097-102.

22. Cao L, Shitara H, Sugimoto M, Hayashi J, Abe K, Yonekawa H. New evidence confirms that the mitochondrial bottleneck is generated without reduction of mitochondrial DNA content in early primordial germ cells of mice. PLoS genetics. 2009;5(12):e1000756.

23. Wai T, Teoli D, Shoubridge EA. The mitochondrial DNA genetic bottleneck results from replication of a subpopulation of genomes. Nat Genet. 2008;40(12):1484-8.

24. Hsu YY, Chou JY. Environmental Factors Can Influence Mitochondrial Inheritance in the Saccharomyces Yeast Hybrids. PLoS One. 2017;12(1):e0169953.

25. Sallevelt SC, de Die-Smulders CE, Hendrickx AT, Hellebrekers DM, de Coo IF, Alston CL, et al. De novo mtDNA point mutations are common and have a low recurrence risk. J Med Genet. 2017;54(2):73-83.

26. Calvo SE, Mootha VK. The mitochondrial proteome and human disease. Annu Rev Genomics Hum Genet. 2010;11:25-44.

27. Prokisch $\mathrm{H}$, Ahting U. MitoP2, an integrated database for mitochondrial proteins. Methods Mol Biol. 2007;372:573-86.

28. Alston CL, Rocha MC, Lax NZ, Turnbull DM, Taylor RW. The genetics and pathology of mitochondrial disease. J Pathol. 2017;241(2):236-50.

29. Ruhoy IS, Saneto RP. The genetics of Leigh syndrome and its implications for clinical practice and risk management. The application of clinical genetics. 2014;7:221-34.

30. Lopez LC, Schuelke M, Quinzii CM, Kanki T, Rodenburg RJ, Naini A, et al. Leigh syndrome with nephropathy and CoQ10 deficiency due to decaprenyl diphosphate synthase subunit 2 (PDSS2) mutations. Am J Hum Genet. 2006;79(6):1125-9.

31. Shoubridge EA. Nuclear genetic defects of oxidative phosphorylation. Hum Mol Genet. 2001;10(20):2277-84.

32. van den Bosch BJ, Gerards M, Sluiter W, Stegmann AP, Jongen EL, Hellebrekers DM, et al. Defective NDUFA9 as a novel cause of neonatally fatal complex I disease. J Med Genet. 2012;49(1):10-5.

33. Pequignot MO, Dey R, Zeviani M, Tiranti V, Godinot C, Poyau A, et al. Mutations in the SURF1 gene associated with Leigh syndrome and cytochrome $C$ oxidase deficiency. Hum Mutat. 2001;17(5):374-81.

34. Rotig A. Genetic bases of mitochondrial respiratory chain disorders. Diabetes \& metabolism. 2010;36(2):97-107.

35. McFarland R, Kirby DM, Fowler KJ, Ohtake A, Ryan MT, Amor DJ, et al. De novo mutations in the mitochondrial ND3 gene as a cause of infantile mitochondrial encephalopathy and complex I deficiency. Ann Neurol. 2004;55(1):58-64.

36. Stroud DA, Surgenor EE, Formosa LE, Reljic B, Frazier AE, Dibley MG, et al. Accessory subunits are integral for assembly and function of human mitochondrial complex I. Nature. 2016;538(7623):123-6.

37. Andrews B, Carroll J, Ding S, Fearnley IM, Walker JE. Assembly factors for the membrane arm of human complex I. Proc Natl Acad Sci U S A. 2013;110(47):18934-9.

38. Copeland WC. Defects in mitochondrial DNA replication and human disease. Critical reviews in biochemistry and molecular biology. 2012;47(1):64-74. 
39. Korhonen JA, Pham XH, Pellegrini M, Falkenberg M. Reconstitution of a minimal mtDNA replisome in vitro. Embo j. 2004;23(12):2423-9.

40. Angelini C, Bello L, Spinazzi M, Ferrati C. Mitochondrial disorders of the nuclear genome. Acta myologica : myopathies and cardiomyopathies : official journal of the Mediterranean Society of Myology. 2009;28(1):16-23.

41. Cohen BH, Chinnery PF, Copeland WC. POLG-Related Disorders. In: Adam MP, Ardinger HH, Pagon RA, Wallace SE, Bean LJH, Mefford HC, et al., editors. GeneReviews((R)). Seattle (WA): University of Washington, Seattle University of Washington, Seattle. GeneReviews is a registered trademark of the University of Washington, Seattle. All rights reserved.; 1993.

42. Wanrooij S, Luoma P, van Goethem G, van Broeckhoven C, Suomalainen A, Spelbrink JN. Twinkle and POLG defects enhance age-dependent accumulation of mutations in the control region of mtDNA. Nucleic Acids Res. 2004;32(10):3053-64.

43. Copeland WC. Inherited mitochondrial diseases of DNA replication. Annual review of medicine. 2008;59:131-46.

44. Chinnery PF, Hudson G. Mitochondrial genetics. British medical bulletin. 2013;106:135-59.

45. Boczonadi V, Horvath R. Mitochondria: impaired mitochondrial translation in human disease. The international journal of biochemistry \& cell biology. 2014;48:77-84.

46. Falkenberg M, Larsson NG, Gustafsson CM. DNA replication and transcription in mammalian mitochondria. Annual review of biochemistry. 2007;76:679-99.

47. Rotig A. Human diseases with impaired mitochondrial protein synthesis. Biochim Biophys Acta. 2011;1807(9):1198-205.

48. Galmiche L, Serre V, Beinat M, Assouline Z, Lebre AS, Chretien D, et al. Exome sequencing identifies MRPL3 mutation in mitochondrial cardiomyopathy. Hum Mutat. 2011;32(11):122531.

49. Gotz A, Tyynismaa H, Euro L, Ellonen P, Hyotylainen T, Ojala T, et al. Exome sequencing identifies mitochondrial alanyl-tRNA synthetase mutations in infantile mitochondrial cardiomyopathy. Am J Hum Genet. 2011;88(5):635-42.

50. Tucker EJ, Hershman SG, Kohrer C, Belcher-Timme CA, Patel J, Goldberger OA, et al. Mutations in MTFMT underlie a human disorder of formylation causing impaired mitochondrial translation. Cell Metab. 2011;14(3):428-34.

51. Szpisjak L, Zsindely N, Engelhardt JI, Vecsei L, Kovacs GG, Klivenyi P. Novel AARS2 gene mutation producing leukodystrophy: a case report. J Hum Genet. 2017;62(2):329-33.

52. Ghezzi D, Baruffini E, Haack TB, Invernizzi F, Melchionda L, Dallabona C, et al. Mutations of the mitochondrial-tRNA modifier MTO1 cause hypertrophic cardiomyopathy and lactic acidosis. Am J Hum Genet. 2012;90(6):1079-87.

53. Scott I, Youle RJ. Mitochondrial fission and fusion. Essays in biochemistry. 2010;47:85-98.

54. Youle RJ, van der Bliek AM. Mitochondrial fission, fusion, and stress. Science (New York, NY). 2012;337(6098):1062-5.

55. Babbar M, Sheikh MS. Metabolic Stress and Disorders Related to Alterations in Mitochondrial Fission or Fusion. Molecular and cellular pharmacology. 2013;5(3):109-33.

56. Hui J, Kirby DM, Thorburn DR, Boneh A. Decreased activities of mitochondrial respiratory chain complexes in non-mitochondrial respiratory chain diseases. Dev Med Child Neurol. 2006;48(2):132-6.

57. Tucker T, Marra M, Friedman JM. Massively parallel sequencing: the next big thing in genetic medicine. Am J Hum Genet. 2009;85(2):142-54.

58. Goldman D, Domschke K. Making sense of deep sequencing. The international journal of neuropsychopharmacology. 2014;17(10):1717-25. 
59. Morozova O, Marra MA. Applications of next-generation sequencing technologies in functional genomics. Genomics. 2008;92(5):255-64.

60. Wang Z, Gerstein M, Snyder M. RNA-Seq: a revolutionary tool for transcriptomics. Nature reviews Genetics. 2009;10(1):57-63.

61. Ellingford JM, Campbell C, Barton S, Bhaskar S, Gupta S, Taylor RL, et al. Validation of copy number variation analysis for next-generation sequencing diagnostics. European journal of human genetics : EJHG. 2017;25(6):719-24. 


\section{CHAPTER 2}

Whole exome sequencing is the preferred, first strategy to identify known and novel causes of mitochondrial disease

Tom E.J. Theunissen, Minh Nguyen, Rick Kamps, Alexandra T. Hendrickx, Suzanne C.E.H. Sallevelt, Ralph W.H. Gottschalk, Chantal M. Calis, Alphons P.M. Stassen, Bart de Koning, Elvira N. M. Mulder-Den Hartog, Kees Schoonderwoerd, Sabine A. Fuchs, Yvonne HilhorstHofstee, Marianne de Visser, Jo Vanoevelen, Radek Szklarczyk, Mike Gerards, Irenaeus F.M. de Coo*, Debby M.E.I. Hellebrekers*, Hubert J.M. Smeets*.

*=joint senior authors

\section{Submitted}




\begin{abstract}
Mitochondrial diseases are clinically and genetically heterogeneous and can be caused by both mitochondrial DNA (mtDNA) and nuclear genetic defects. We have performed a two-step next-generation sequencing-based approach in a cohort of 117 patients, mostly children, with either a high suspicion of a mitochondrial disorder or with a disease phenotype in which mitochondrial defects are part of the differential diagnosis. Patients were screened for mtDNA defects and, if excluded, analysed by whole exome sequencing (WES). We filtered for autosomal recessive and $X$-linked recessive mutations in families with multiple affected siblings and/or consanguineous parents. Non-consanguineous families with a single patient were additionally screened for autosomal and X-linked dominant mutations in a predefined gene set. We detected and quantified mtDNA point-mutations and indels in $21 \%$ of the patient-cohort, and identified disease-causing exonic mutations in $49 \%$ of the patients, implying an overall diagnostic yield of $69 \%$. We show that $30 \%$ of the disease-causing genes in mitochondrial WES-patients were not in the MitoCarta database at the time of discovery, and therefore would have been missed with targeted sequencing approaches. WES is a comprehensive and unbiased approach to establish a genetic diagnosis in mitochondrial disease and able to fully resolve heterogeneous and multi-genic disease manifestations.
\end{abstract}




\section{Introduction}

Mitochondrial disorders are most often characterized by deficiencies in oxidative phosphorylation (OXPHOS) and ATP production, and manifest with a broad spectrum of clinical symptoms. A single gene defect can demonstrate a broad and complex variety of symptoms (clinical heterogeneity) and many different gene defects can result in a similar phenotype (genetic heterogeneity). Characterization of the genetic basis of mitochondrial disease is further complicated due to the involvement of both the nuclear and mitochondrial genomes \{Rotig, $2003 \# 561\}(1,2)$. For pathogenic mutations in the multi-copy mtDNA, which contains 37 genes and is exclusively maternally inherited, the percentage of socalled heteroplasmic mutations also affects the clinical presentation $(3,4)$. The estimated number of nuclear genes involved in mitochondrial function is around $1500(5,6)$, of which only $>250$ genes have been shown to be involved in mitochondrial disease (7). In primary mitochondrial disorders (MD) the OXPHOS deficiencies are often due to genetic defects in the OXPHOS complexes (subunits and assembly factors), or, more indirectly, in processes such as mitochondrial protein translation and degradation, mtDNA maintenance, fusion and fission, substrate transport or phospholipid metabolism. Still, mitochondrial dysfunction can also be a more secondary defect in genetic syndromes or neuromuscular disease (8). Finally, for a broad variety of clinical entities, a mitochondrial gene defect can explain a minority of cases. These were analyzed as well by WES and referred to as differential diagnosis (DD).

Genetic diagnosis of mtDNA-disorders requires screening of all 37 mtDNA genes, determining heteroplasmy levels of variants and characterizing large mtDNA deletions and mtDNA copy number. mtDNA analysis in diagnostic setting commonly started with screening for a few relatively common point mutations using mutation-specific restriction enzymes or qPCR based methods $(9,10)$. In case common mutations were not detected, the entire mitochondrial genome was analysed by Sanger sequencing or chip-based methods (11, 12). However, these methods are non-quantitative, requiring a second molecular test to determine the mutation load. Besides, chip-based methods have difficulties in detecting small indels (13-15). For the large mtDNA deletions, it was time-consuming to determine the exact breakpoints, which could be important for prognosis $(16,17)$. The application of next-generation sequencing has greatly increased the possibilities for detecting and quantifying point mutations and indels across the complete mitochondrial genome and for characterizing large mtDNA deletions with one single technology (18-20). Although, accurate determination of the heteroplasmy levels of large mtDNA deletions and mtDNA copy number still require quantitative PCR analysis. If no mtDNA mutations are present, then moving into the analysis of nuclear genes traditionally relied on (stepwise) Sanger sequencing of nuclear candidate genes which were selected based on clinical and biochemical features 
or linkage/homozygosity mapping. Development of next-generation sequencing methods, such as WES, enable the detection of the majority of the genetic variations in the coding part of the genome (21). An unbiased and complete genetic analysis is important, especially in heterogeneous mitochondrial disease, where genotype-phenotype relations can be indistinct and novel genes involved.

We have performed a complete next-generation sequencing strategy, analysing the mtDNA and exome in a cohort of 84 patients with primary mitochondrial disease and 33 patients where a mitochondrial cause is part of the differential diagnosis. The results were obtained over a 4-year period from 2012 to 2016. Patients were tested for mtDNA mutations by nextgeneration sequencing and, if negative, further analysed by WES. WES-variants were filtered according to the presumed genetic model of disease inheritance, allele frequencies and the effect and conservation of the variant. With this approach, we identified in about $70 \%$ of our patients a causative mutation in new and known disease genes, which were either inherited or de novo.

\section{Material and Methods}

\section{Patients}

117 patients, from consanguineous and non-consanguineous families, in this study were under treatment at Erasmus MC, Maastricht UMC+, Leiden UMC, UMC Utrecht, AMC Amsterdam (The Netherlands), and informed consent was obtained for WES analysis. Research was prospectively reviewed and approved by the local ethical committee of the Maastricht University Medical Centre. Patients were classified as having a "mitochondrial disorder" (MD) if their profile met clinical criteria and/or biochemical/genetic criteria. Patients not meeting these criteria were part of the differential diagnostic (DD) procedure. MD is based on:

- Clinical criteria: Mitochondrial syndromes (LHON, CPEO/KSS, MERRF, MIDD/MELAS, Leigh syndrome, Miller- and Barth-syndrome). Patients were considered mitochondrial, if symptom profiles were scored as "probably" (5-7 points) or "definite" (8-12 points) mitochondrial disease, according to the "Nijmegen Clinical Criteria for Mitochondrial Disease".

- Biochemical criteria: A decreased mitochondrial respiratory capacity in muscle tissue or fibroblasts, measured as significantly decreased OXPHOS complex activity (isolated or combined OXPHOS deficiencies), reduced oxygen consumption, and/or decreased ATP production.

- Genetic criteria: Presence of mtDNA depletion or multiple mtDNA deletions 


\section{mtDNA analysis}

Sequencing of the mtDNA was performed using the Illumina MiSeq platform. Enrichment of the entire mtDNA was performed by a single long-range PCR using Phusion Hot Start DNA polymerase II kit (Thermoscientific) and $100 \mathrm{ng}$ of total genomic DNA, according to the manufacturer's instructions. Library preparation was performed by the Illumina Nextera XT kit according to the manufacturer's instructions. Twelve indexed DNA libraries were equimolarly pooled and sequenced in a single lane of 1 MiSeq flow-cell with a 2x300bp paired-end chemistry. Data demultiplexing was performed with Illumina CASAVA software (v.1.8.4.) and reads were aligned against the revised Cambridge mitochondrial reference sequence (but without the gap at position 3107) by BWA software (v.0.5.9.) (22). For both variant and small indel identification, Python 2.6.6., Python package pysam 0.7.8. and SAMTools 0.1.19 software were used (23). Large deletions were identified by alignment with the Smith and Waterman algorithm and the EMBOSS water program (v.6.5.7.). Annotation and filtering of mtDNA variants and indels were performed using in-house build Perl tools and a MySQL annotation database. Calculation of the heteroplasmy level at any nucleotide position was performed by the read depth of the mutant versus reference nucleotide. We have validated the detection and quantification accuracy of our NGS strategy by analysing DNA samples with varying heteroplasmy levels of different substitution mutations, small indels and large deletions, as previously determined by MitoChip, RFLP and PCR/Southern blotting (data available on request). The entire mtDNA, excluding the highly polymorphic D-loop, was analysed using a $2 \%$ heteroplasmy cut-off for known pathogenic point mutations and a $5 \%$ cut-off for the remaining positions and small indels. Heteroplasmy levels of large mtDNA deletions were determined by qPCR, using SensiMix SYBR (Bioline). Also to identify mtDNA depletion in muscle biopsies (if available), qPCR quantification was performed, where mtDNA copy-number (based on MT-ND1) was normalized to the nDNA copy-number (based on B2M).

\section{Homozygosity Mapping and WES}

Homozygosity mapping and CNV analysis was performed by HumanMapping 250K array (Affymetrix, Santa Clara, California) and Genotyping console 4.0 (Affymetrix). Homozygosity or hemizygosity regions were defined by the "Homozygosity" mapper (24), with a cutoff of $5 \mathrm{MB}$. Exons were captured with SureSelect version 5 exome enrichment kit (Agilent Technologies, Santa Clara, California), including untranslated regions. Sequencing was performed on a HiSeq2000 platform (Illumina, San Diego, California), using a $2 \times 100 \mathrm{bp}$ paired-end setting. Bcl2fastq 1.8.4 (Illumina) allowed Basecalling and demultiplexing, and Burrows-Wheeler Aligner 0.5.9 (Broad Institute, Cambridge, Massachusetts) was used for 
read alignment against human reference genome hg19. Duplicate reads were removed by Picard software suite 1.77 (Broad Institute, Cambridge, Massachusetts) and variant calling was performed with GATK 2.1-8 (Broad Institute).

Exome data of consanguineous families or families consisting of $>1$ patient was filtered for recessive homozygous, compound heterozygous and X-linked (XLR) mutations. Variants with allele frequencies $<1 \%$ (dbSNP137, Exome Aggregation Consortium) were evaluated, covering missense mutations, indels, nonsense mutations, and splice variants. Nonannotated variants were maintained, unless allele frequencies exceeded $5 \%$ prevalence in our in-house patient database. Pathogenicity of nonsynonymous missense mutation was estimated by Polymorphism Phenotyping-2 (PolyPhen-2; Harvard, Boston, Massachusetts), Sorting Intolerant From Tolerant (J. Craig Venter Institute, Rockville, Maryland), Protein Variation Effect Analyzer (PROVEAN; J. Craig Venter Institute), and MutationTaster (NeuroCure Cluster of Excellence/Berlin Institute of Health, Berlin, Germany). Nonsense, frameshift, and splice-site variations were maintained. WES data of single patients from nonconsanguineous families were additionally filtered for heterozygous mutations in known (OMIM (Online Mendelian Inheritance in Man) disease genes with known dominant and/or de novo mutations. All identified pathogenic mutations were checked for their inclusion in the MitoCarta database for mitochondrial localized proteins.

\section{Results}

\section{Diagnostic yield}

A cohort of $117 \mathrm{MD}$ and DD index-patients from unaffected parents, was subject to a twostep next-generation sequencing approach (Figure 1). The age of disease onset in the cohort varied from birth to approximately 50 years old, 78\% (91/117) were paediatric patients (<18 years of age). We solved $21 \%$ of the cohort with a disease-causing mtDNA defect, involving 24 MD-patients. Subsequent WES analysis solved an additional 49\% (57 patients) of the cohort, consisting of $37 \mathrm{MD}$-patients and 20 DD-patients. With this strategy, we achieved an overall diagnostic yield of $69 \%$, with comparable yield in the group of inherited disease cases (70\%) compared to the single patients from non-consanguineous families (68\%). 


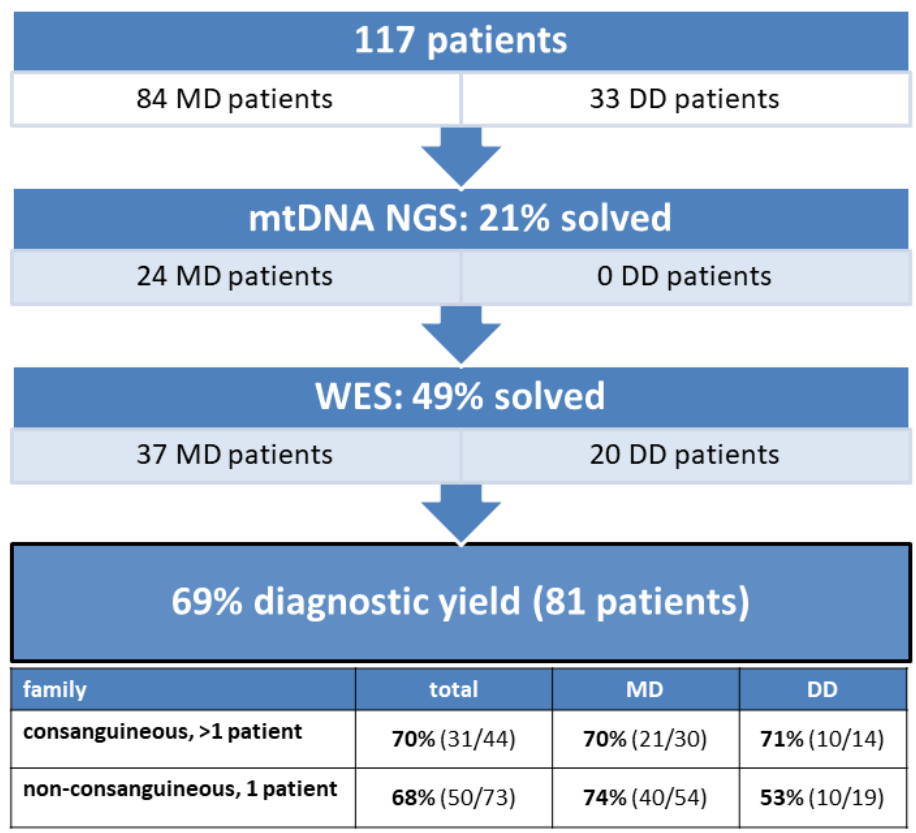

Figure 1: The diagnostic yield of mtDNA and whole exome sequencing in a patient cohort consisting of 117 MD and DD patients. 21\% of the patients were solved with a mtDNA defect and $49 \%$ with a nuclear DNA defect, implying an overall diagnostic yield of $69 \%$.

\section{mtDNA next-generation sequencing}

mtDNA sequencing resulted in the identification of a disease-causing mutation in $21 \%$ $(24 / 117)$ of the patient cohort (Figure 1$)$, solving $5 \%(2 / 44)$ of the inherited disease cases (consanguineous, $>1$ patient) and $30 \%$ (22/73) of the single patients from non-consanguineous families. As shown in Table 1, all cases with a mtDNA defect were MD-patients and all 24 identified mtDNA defects involved known mtDNA mutations that have been reported in LHON (Leber's hereditary optic neuropathy), MELAS (mitochondrial encephalomyopathy, lactic acidosis, and stroke-like episodes)/MIDD (maternally inherited diabetes mellitus and deafness), MERRF (myoclonic epilepsy with ragged red fibers), Leigh syndrome and CPEO (chronic progressive external ophthalmoplegia) /Kearns-Sayre syndrome. 38\% (9/24) of the mtDNA mutations were primary, mostly (8 out of 9 ) homoplasmic, LHON mutations (m.11778G $>$ A and m.14484T>C), clinically manifesting with optic atrophy. Only one LHON patient carried $80 \%$ heteroplasmy. The m.3243A>G mutation covered $29 \%(7 / 24)$ of the mtDNA defects, and explained both MELAS ( 2 patients) and MIDD (5 patients) phenotypes. 
Table 1: Disease-causing mutations identified by mtDNA sequencing. mtDNA point-mutations and deletions were detected and quantified by mtDNA next-generation sequencing in a cohort of 117 patients. All patients with a mtDNA defect were classified as MD patients.

\begin{tabular}{|c|c|c|c|c|c|c|c|}
\hline & & family & patients & symptoms & mutation & $\begin{array}{l}\text { heteroplasmy } \\
\text { (in blood) }\end{array}$ & gene \\
\hline \multirow{2}{*}{ 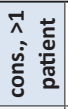 } & \multirow{2}{*}{$\stackrel{0}{\Sigma}$} & non-consanguineous & 2 & LHON & m.11778G >A & homoplasmic & MT-ND4 \\
\hline & & non-consanguineous & 2 & LHON & m.11778G $>A$ & homoplasmic & MT-ND4 \\
\hline \multirow{22}{*}{ 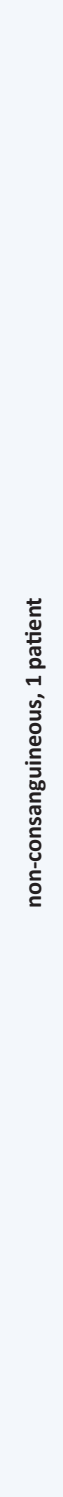 } & \multirow{22}{*}{$\stackrel{0}{\Sigma}$} & non-consanguineous & 1 & MERRF & $\mathrm{m} .8344 \mathrm{~A}>\mathrm{G}$ & $10 \%$ & MT-TK \\
\hline & & non-consanguineous & 1 & MELAS & $\mathrm{m} .3243 \mathrm{~A}>\mathrm{G}$ & $24 \%$ & MT-TL1 \\
\hline & & non-consanguineous & 1 & MELAS & $\mathrm{m} .3243 \mathrm{~A}>\mathrm{G}$ & $41 \%$ & MT-TL1 \\
\hline & & non-consanguineous & 1 & $\begin{array}{l}\text { MIDD, ptosis, macular } \\
\text { degeneration }\end{array}$ & $\mathrm{m} .3243 \mathrm{~A}>\mathrm{G}$ & $25 \%$ & MT-TL1 \\
\hline & & non-consanguineous & 1 & MIDD & m. $3243 A>G$ & $26 \%$ & MT-TL1 \\
\hline & & non-consanguineous & 1 & MIDD & $\mathrm{m} .3243 \mathrm{~A}>\mathrm{G}$ & $10 \%$ & MT-TL1 \\
\hline & & non-consanguineous & 1 & $\begin{array}{l}\text { MIDD, macular } \\
\text { degeneration }\end{array}$ & m. $3243 A>G$ & $7 \%$ & MT-TL1 \\
\hline & & non-consanguineous & 1 & MIDD, cardiomyopathy & m. $3243 A>G$ & $40 \%$ & MT-TL1 \\
\hline & & non-consanguineous & 1 & Leigh syndrome & m.13513G >A & $72 \%$ & MT-ND5 \\
\hline & & non-consanguineous & 1 & LHON & m.11778G $>A$ & $80 \%$ & MT-ND4 \\
\hline & & non-consanguineous & 1 & LHON & m.11778G >A & homoplasmic & MT-ND4 \\
\hline & & non-consanguineous & 1 & LHON & m.11778G $>A$ & homoplasmic & MT-ND4 \\
\hline & & non-consanguineous & 1 & LHON & m.11778G>A & homoplasmic & MT-ND4 \\
\hline & & non-consanguineous & 1 & LHON & m.11778G >A & homoplasmic & MT-ND4 \\
\hline & & non-consanguineous & 1 & LHON & m.14484T $>C$ & homoplasmic & MT-ND6 \\
\hline & & non-consanguineous & 1 & LHON & m.14484T >C & homoplasmic & MT-ND6 \\
\hline & & non-consanguineous & 1 & CPEO & $\begin{array}{l}\text { de novo: single } \\
8284 \text { bp deletion } \\
\text { (7462:15747) }\end{array}$ & n.d. & covers 22 mtDNA genes \\
\hline & & non-consanguineous & 1 & CPEO & $\begin{array}{c}\text { de novo: single } \\
6277 \text { bp deletion } \\
\text { (9514-15792) }\end{array}$ & n.d. & $\begin{array}{c}\text { MT-CO3, MT-ND3, MT-ND4L, } \\
\text { MT-ND4, MT-ND5, MT-ND6, } \\
\text { MT-CYB, tRNA's (Leu, Ser, } \\
\text { His, Gly, Arg, Glu) }\end{array}$ \\
\hline & & non-consanguineous & 1 & CPEO & $\begin{array}{l}\text { de novo: single } \\
4977 \text { bp deletion } \\
\text { (breakpoints } \\
8482: 13460 \text { ) }\end{array}$ & n.d. & $\begin{array}{c}\text { MT-ATP8, MT-ATP6, MT-CO3, } \\
\text { MT-ND3, MT-ND4L, MT-ND4, } \\
\text { MT-ND5, tRNA's (Leu, Ser, } \\
\text { His, Gly, Arg) }\end{array}$ \\
\hline & & non-consanguineous & 1 & CPEO & $\begin{array}{l}\text { de novo: single } \\
4977 \text { bp deletion } \\
\text { (breakpoints } \\
8482: 13460 \text { ) }\end{array}$ & n.d. & $\begin{array}{c}\text { MT-ATP8, MT-ATP6, MT-CO3, } \\
\text { MT-ND3, MT-ND4L, MT-ND4, } \\
\text { MT-ND5, tRNA's (Leu, Ser, } \\
\text { His, Gly, Arg) }\end{array}$ \\
\hline & & non-consanguineous & 1 & CPEO & $\begin{array}{l}\text { de novo: single } \\
4977 \text { bp deletion } \\
\text { (breakpoints } \\
8482: 13460 \text { ) }\end{array}$ & n.d. & $\begin{array}{c}\text { MT-ATP8, MT-ATP6, MT-CO3, } \\
\text { MT-ND3, MT-ND4L, MT-ND4, } \\
\text { MT-ND5, tRNA's (Leu, Ser, } \\
\text { His, Gly, Arg) }\end{array}$ \\
\hline & & non-consanguineous & 1 & Kearns-Sayre syndrome & $\begin{array}{l}\text { de novo: single } \\
4977 \text { bp deletion } \\
\text { (breakpoints } \\
8482: 13460 \text { ) }\end{array}$ & n.d. & $\begin{array}{c}\text { MT-ATP8, MT-ATP6, MT-CO3, } \\
\text { MT-ND3, MT-ND4L, MT-ND4, } \\
\text { MT-ND5, tRNA's (Leu, Ser, } \\
\text { His, Gly, Arg) }\end{array}$ \\
\hline
\end{tabular}


Other mutations were identified in the ND5 gene (m.13513G $>A)$ and the tRNA-Lysine gene (m.8344A>G), as a cause of Leigh syndrome and MERRF, respectively. 25\% (6/24) of the mtDNA defects were large single deletions, of which the $4977 \mathrm{bp}$ deletion (breakpoints $8482: 13460$ ) was detected in 4 patients. These patients had CPEO, sometimes in combination with additional symptoms of Kearns-Sayre syndrome (pigmentary retinopathy, cardiac conduction abnormalities). Patients with multiple mtDNA deletions (not included in Table 1) were further analysed by WES, as being suggestive of a underlying nuclear DNA defect in the mtDNA maintanance or replication genes.

\section{Whole Exome Sequencing}

In the remaining $93 \mathrm{MD}$ and DD patients, the presence of pathogenic mtDNA mutations was excluded. Prior to whole exome analysis, these patients were subject to SNP-array analysis, using the Affymetrix GeneChip Human Mapping 250K, to detect copy number variations (CNV) and homozygosity regions. No CNVs were detected. In patients from consanguineous families, mapping of the homozygous regions provided an additional filtering criterion to select the most promising genetic variants from the exome data. When multiple affected siblings were present, the entire family, if available, was analysed by means of SNP-arrays. This approach significantly increased the efficiency of interpreting WES results. WES identified a disease-causing gene defect in $49 \%$ (57/117) of the complete patient cohort (Figure 1). In an additional $7 \%$ of the cohort, WES analysis identified a genetic variant which could explain the patient's phenotype, but for which definite evidence is currently lacking. Further studies should reveal which of these mutations could be classified as disease-causing.

WES-data filtering was based on the presumed genetic mode of inheritance. We applied a variant selection strategy for autosomal recessive (AR) and recessive $X$-linked (XLR) disorders to the inherited disease cases (consanguinity, $>1$ patient). As shown in Table 2 , this group consisted of 42 families, where WES identified of a genetic defect in 69\% (29/42) of the cases, covering homozygous, compound heterozygous and X-linked mutations (Table 3). In MD patients, 68\% (19/28) of the cases were solved, and in the DD group a disease causing gene defect was found in $71 \%$ (10/14). A broad spectrum of genes with relatively few primary mitochondrial genes $(10 \%(1 / 10))$ was identified among the DD patients compared to the proportion of primary mitochondrial genes in MD patients (71\% (15/21)) (Table 3). In 51 non-consanguineous families with a single patient, we applied a variant selection strategy for autosomal recessive (AR), X-linked (XLR and XLD) and single heterozygous mutations, expected to be dominant de novo mutations. In order to limit the huge amount of heterozygous variants for dominant disease-causing mutations, variant interpretation was restricted to genes that have previously been reported in dominant 
disease based on the OMIM database. With this approach, we unravelled the genetic cause in $55 \%(28 / 51)$ of these families (Table 2 ), $16 \%$ of which were single dominant mutations. Follow-up investigations in the parents revealed that the mutations either occurred de novo in the patients or were present in one of the parent, who displayed subclinical symptoms upon further investigation. The diagnostic yield was $56 \%(18 / 32)$ in the MD patient group and $53 \%(10 / 19)$ in the DD patient group, where respectively, $89 \%(17 / 19)$ and $18 \%(2 / 11)$ of the defects were located in a gene with reported mitochondrial function (Table 3 ).

Table 2: Whole-exome sequencing analysis. 93 patients were subject to WES analysis. WES-data was filtered according to the presumed pattern of disease inheritance, where consanguinity or involvement of $>1$ patient were indicative of a recessively inherited disorder and non-consanguineous families with a single patient were likely to cover both, recessively inherited and dominant de novo mutations.

\begin{tabular}{|c|c|c|c|}
\hline WES filtering strategy & $\begin{array}{c}\text { Total } \\
\% \text { solved }\end{array}$ & $\begin{array}{c}\text { MD } \\
\% \text { solved }\end{array}$ & $\begin{array}{c}\text { DD } \\
\text { \% solved }\end{array}$ \\
\hline $\begin{array}{cc}\text { consanguineous and/or }>\mathbf{1} \text { patient } \\
-\quad \text { autosomal recessive (AR) } \\
-\quad \text { X-linked (XLR) }\end{array}$ & $69 \%(29 / 42)$ & $68 \%(19 / 28)$ & $71 \%(10 / 14)$ \\
\hline $\begin{array}{cc}\text { non-consanguineous, } 1 \text { patient } \\
-\quad \text { autosomal recessive (AR) } \\
-\quad \text { X-linked (XLR and XLD) } \\
-\quad \text { autosomal dominant (AD) }\end{array}$ & $\begin{array}{l}55 \%(28 / 51) \\
\text { of which } 16 \% \text { de } \\
\text { novo }(8 / 51)\end{array}$ & $56 \%(18 / 32)$ & $53 \%(10 / 19)$ \\
\hline
\end{tabular}

Overall, we saw that $30 \%(12 / 40)$ of the disease-causing genes in the MD patients were not included in the MitoCarta database for mitochondrial localized proteins at the time we identified the genetic defect (Table 3). Surprisingly, this included 4 genes with a literature reported function in mitochondrial metabolism ( $Q R S L 1, R R M 2 B$, c19orf12, $T A Z)$. Furthermore, we identified 5 different genes with, so far, no reported function in mitochondrial metabolism (IER3IP1, SLC19A3 (2x), SLC16A2, IARS, CHRNE) to be involved in mitochondrial disease. Two additional genes that were lacking in the MitoCarta database were most likely not related to the mitochondrial symptoms of the patients, as these nonmitochondrial genes were, together with a primary mitochondrial gene defect, part of a complex multi-genic disease phenotype (ACY1, ANTXR2). 


\begin{tabular}{|c|c|c|c|c|c|c|c|c|c|c|c|c|c|c|c|}
\hline 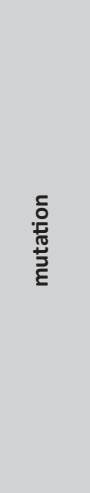 & 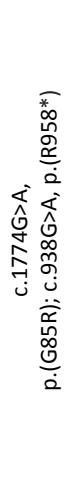 & 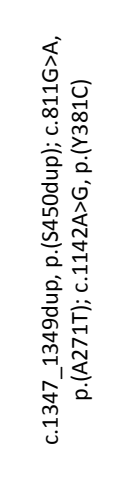 & 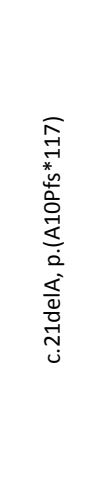 & 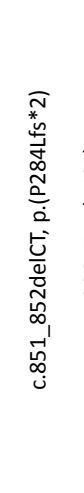 & 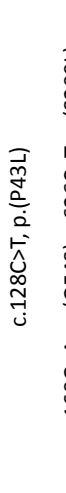 & 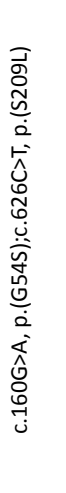 & 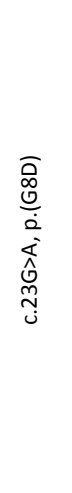 & 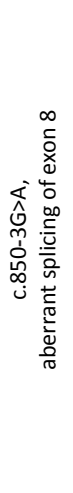 & 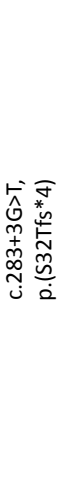 & 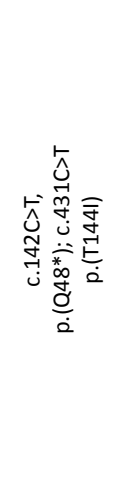 & 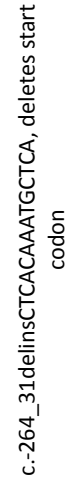 & 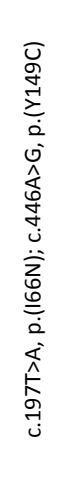 & 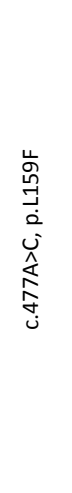 & 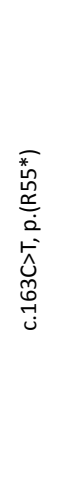 & 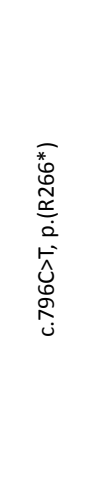 \\
\hline 怘 & ָे & 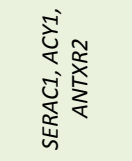 & ఏ & 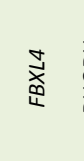 & 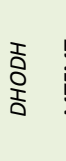 & $\underset{\substack{k \\
\Sigma}}{\stackrel{k}{\Sigma}}$ & $\begin{array}{l}\frac{\pi}{4} \\
\frac{1}{2} \\
\frac{1}{2}\end{array}$ & సี่ & 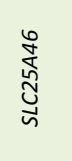 & $\sum_{\substack{\infty \\
\ll}}^{\infty}$ & 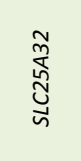 & ơ & $\begin{array}{l}\frac{h}{4} \\
\frac{4}{2} \\
\frac{1}{2}\end{array}$ & 志 & $\frac{\pi}{2}$ \\
\hline 䒿 & $\stackrel{\dddot{\Perp}}{\curvearrowright}$ & 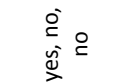 & $\stackrel{\dddot{\Perp}}{\curvearrowright}$ & $\stackrel{\dddot{\jmath}}{>}$ & $\stackrel{\dddot{\nu}}{\supset}$ & $\stackrel{\dddot{\Perp}}{\curvearrowright}$ & $\stackrel{\dddot{\Perp}}{\curvearrowright}$ & 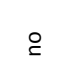 & $\stackrel{\check{\Perp}}{\curvearrowright}$ & 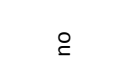 & $\stackrel{\check{\Perp}}{\curvearrowright}$ & $\stackrel{\dddot{\Perp}}{>}$ & $\stackrel{\check{\Perp}}{\curvearrowright}$ & $\stackrel{\check{\Perp}}{\curvearrowright}$ & 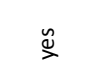 \\
\hline 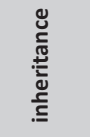 & $\stackrel{\frac{q}{4}}{2}$ & $\begin{array}{l}\frac{r}{4} \\
\frac{a}{4} \\
\frac{a}{4}\end{array}$ & $\frac{⿱}{4}$ & $\stackrel{\ltimes}{<}$ & $\stackrel{q}{<}$ & $\frac{⿱}{4}$ & 孚 & $\stackrel{\frac{a}{4}}{2}$ & 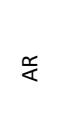 & $\stackrel{\frac{a}{4}}{2}$ & $\stackrel{\ltimes}{<}$ & $\frac{a}{4}$ & $\stackrel{\frac{\alpha}{4}}{2}$ & $\stackrel{\frac{9}{4}}{2}$ & $\stackrel{\frac{\alpha}{4}}{2}$ \\
\hline 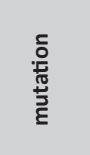 & 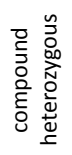 & 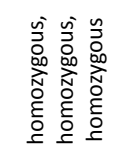 & 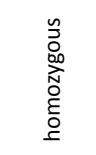 & 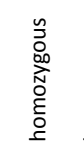 & 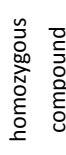 & 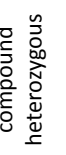 & 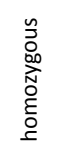 & 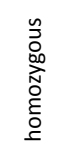 & 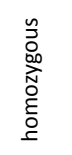 & 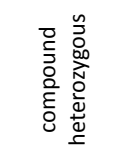 & 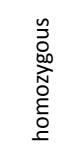 & 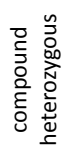 & 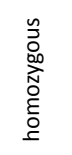 & 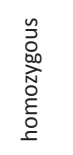 & 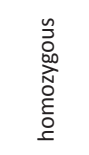 \\
\hline 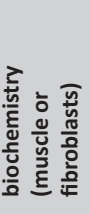 & 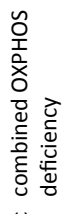 & $\stackrel{\overline{0}}{\bar{g}}$ & 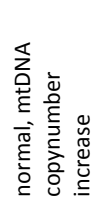 & 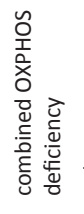 & 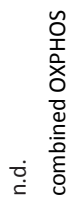 & 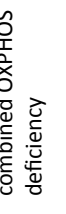 & 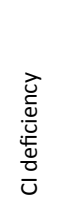 & 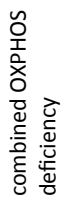 & 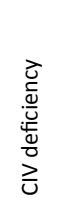 & 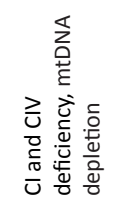 & 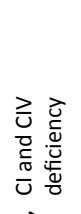 & 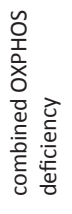 & 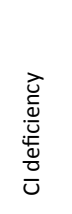 & 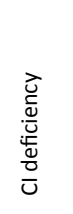 & 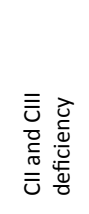 \\
\hline 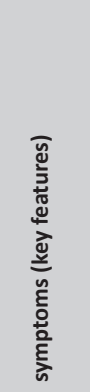 & 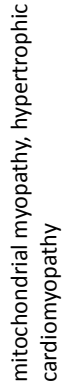 & 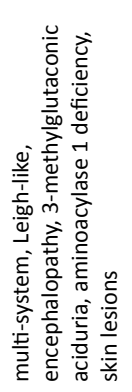 & 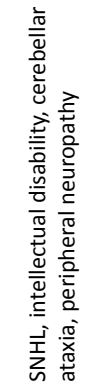 & 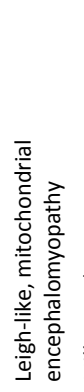 & 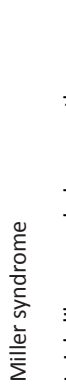 & 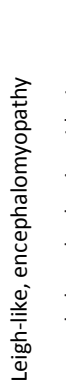 & 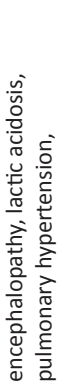 & 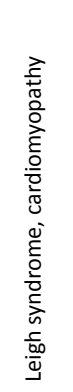 & 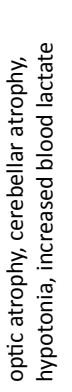 & 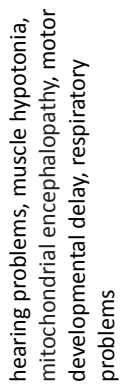 & 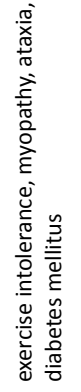 & 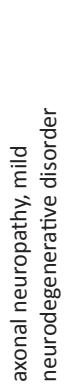 & 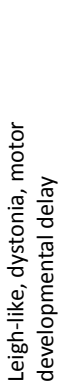 & 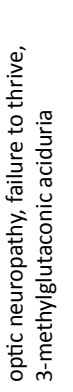 & 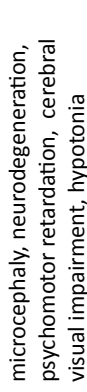 \\
\hline 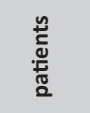 & $m$ & 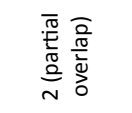 & $\sim$ & n & n & m & $r$ & $\sim$ & -1 & $r$ & $\rightarrow$ & $\sim$ & 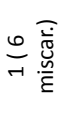 & $\rightarrow$ & $m$ \\
\hline \multirow[t]{3}{*}{ 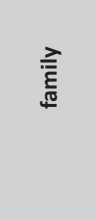 } & 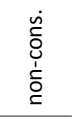 & $\dot{\tilde{c}}$ & $\dot{\tilde{c}}$ & $\dot{\tilde{c}}$ & $\begin{array}{l}\dot{c} \\
\bar{\sigma}\end{array}$ & 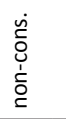 & $\begin{array}{l}\dot{\tilde{c}} \\
\overline{\tilde{u}}\end{array}$ & $\begin{array}{l}\dot{\mathrm{c}} \\
\overline{\tilde{u}}\end{array}$ & $\dot{\tilde{c}}$ & 它 & $\dot{\tilde{c}}$ & $\begin{array}{l}\dot{0} \\
\dot{0} \\
\dot{0} \\
\dot{1} \\
\dot{0} \\
\end{array}$ & $\dot{\check{c}}$ & $\dot{\vec{c}}$ & $\dot{\tilde{c}}$ \\
\hline & \multicolumn{15}{|c|}{ aw } \\
\hline & \multicolumn{15}{|c|}{ 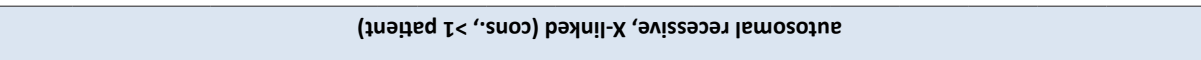 } \\
\hline
\end{tabular}




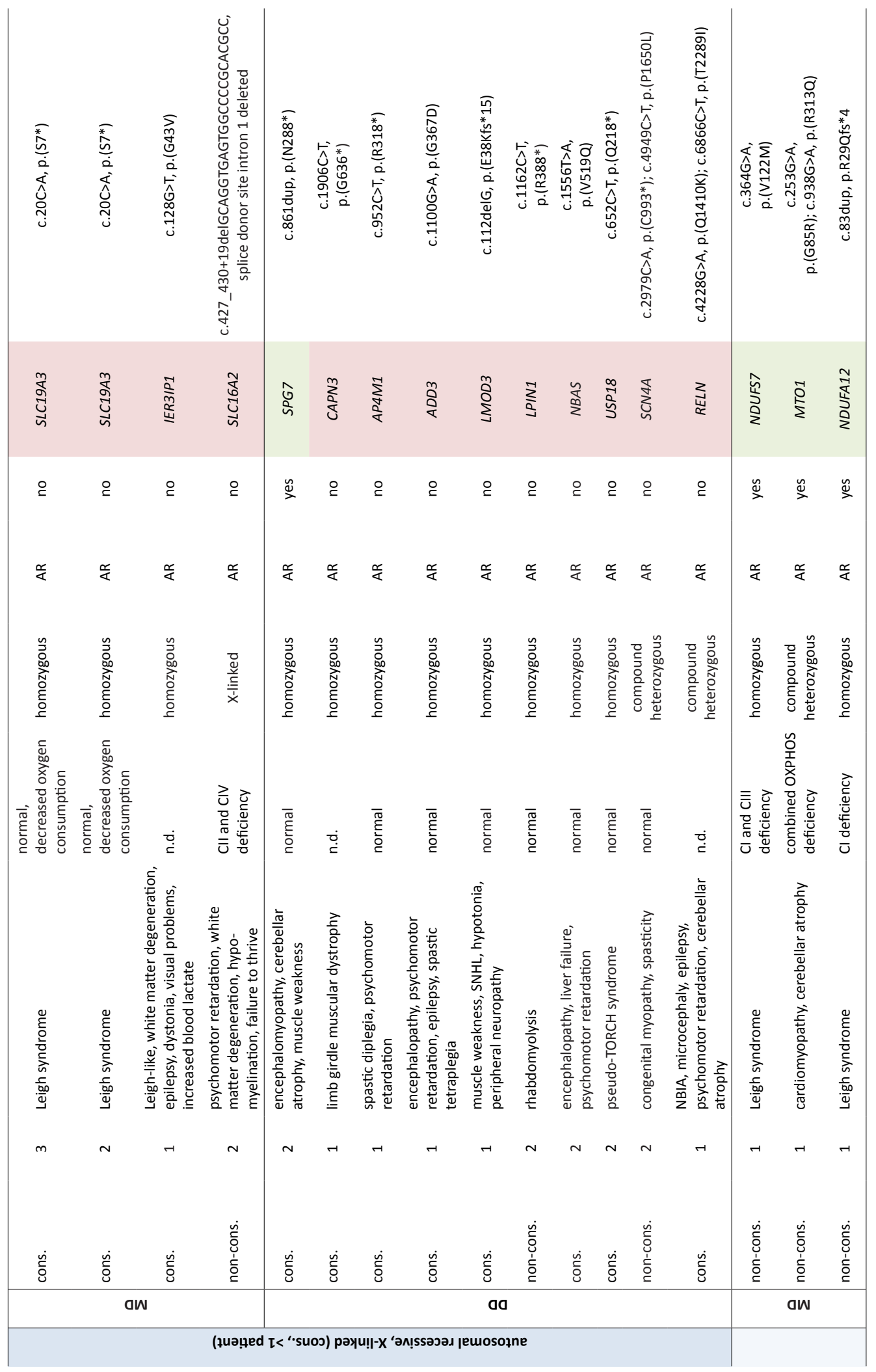




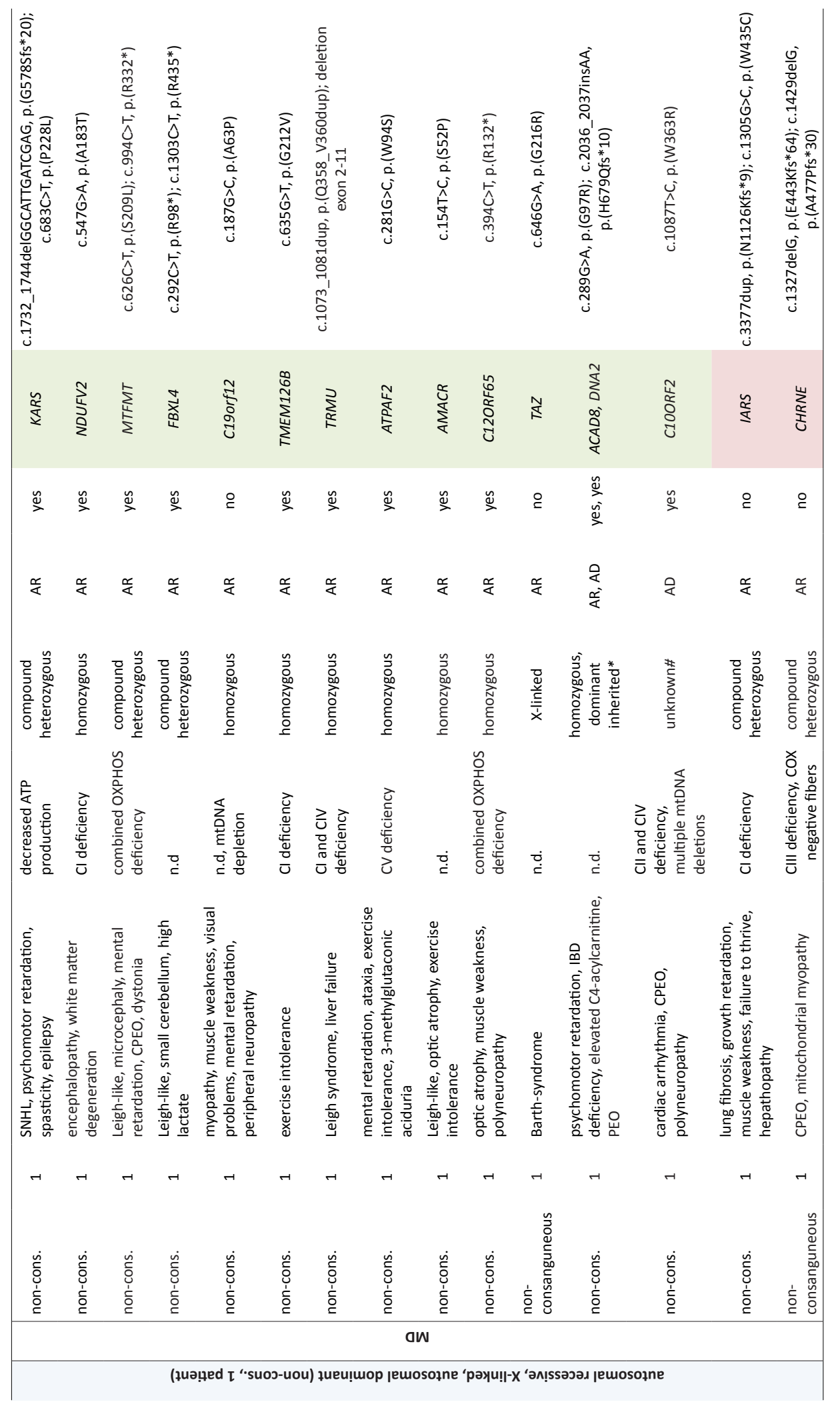




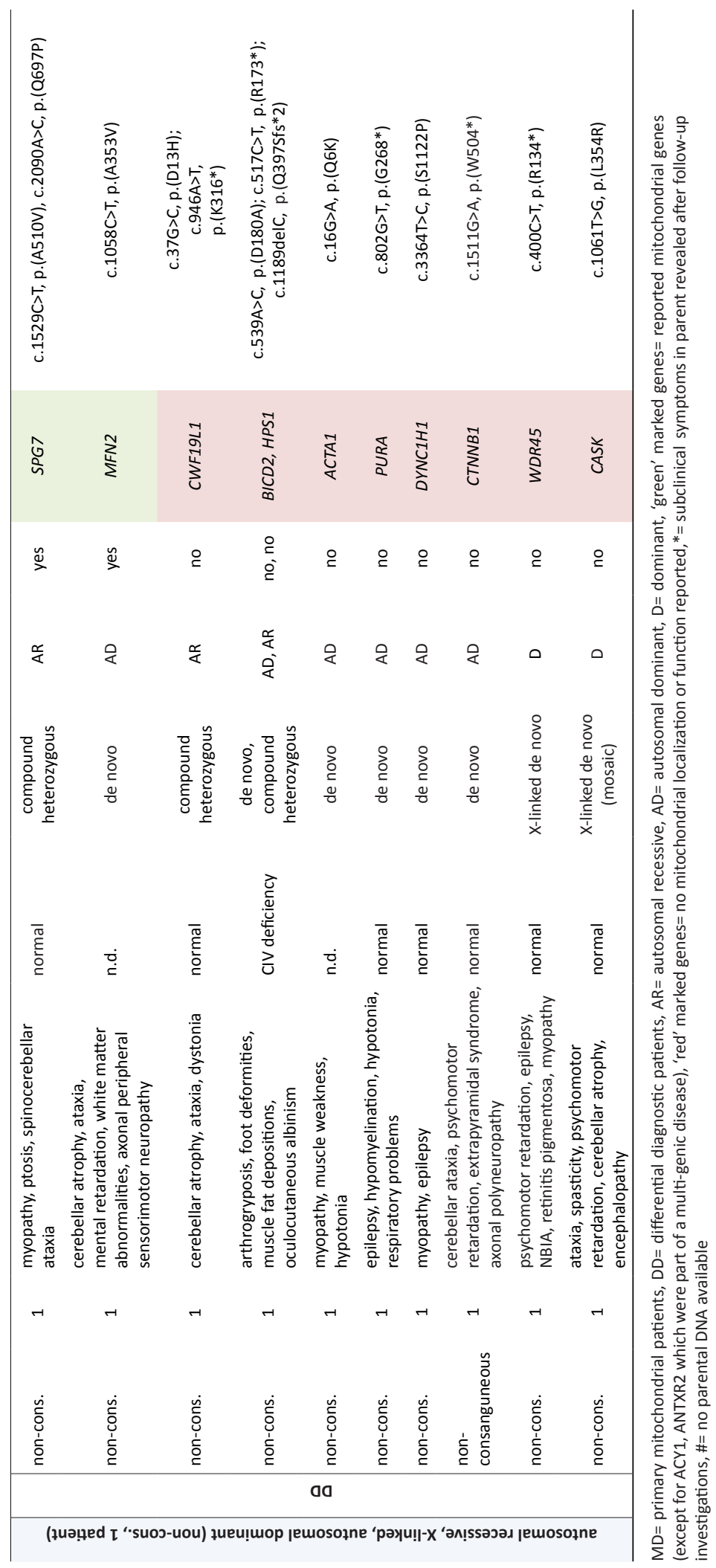


Genes identified in MD patients were clustered according to their function (Table 4). Most genetic defects were detected in genes related to mitochondrial protein metabolism (protein translation and degradation) and OXPHOS function (ETC subunits, assembly factors and cofactors). The first group mainly consisted of mitochondrial tRNA synthethases, transferases and modification enzymes, where the resulting defects in mitochondrial protein synthesis (AARS2, MTFMT, MTO1, QRSL1, TRMU, C12ORF65) especially manifested with combined OXPHOS deficiencies (not measured for KARS). Among the group of OXPHOS-associated genes, defects in complex I subunits and assembly factors (NDUFA12, NDUFS7, NDUFV2, TMEM126A, TMEM126B, NDUFAF4, NDUFAF5) were most prevalent, all resulting in a significant complex I deficiency at the biochemical level. NDUFS7 resulted in an additional complex III deficiency. Defects in NDUFS7, NDUFA12 and NDUFAF5 caused Leigh-syndrome or a Leigh-like-phenotype. The third largest group represented genes involved in mtDNA metabolism, required for mtDNA maintenance, replication and nucleotide metabolism. The $R R M 2 B$ defect resulted in mtDNA depletion, whereas mutations in the RMM2B interacting protein PYCR2 did not reveal any mtDNA abnormalities. Furthermore, mutations in c10orf2 (twinkle) and DNA2 displayed multiple mtDNA deletions in muscle. Interestingly, we found the mitochondrial localized, but functionally uncharacterized, c19orf12 gene to be associated with mtDNA depletion. Among the MD patients, we identified mutations in 5 genes without a reported mitochondrial function or localization, but reported to result in a comparable phenotype as in our patient. 4 genes, functional in different cellular processes, including the transporter proteins SLC19A3 (thiamine) and SLC16A2 (thyroid hormones), tRNA-synthetase IARS, and acetylcholine receptor subunit CHRNE, were likely to underlie the OXPHOS deficiencies measured in these patients. In an additional patient, the functionally uncharacterized IER3IP1 gene was likely to explain the seemingly mitochondrial disease phenotype, yet no OXPHOS measurement was performed in this patient. As indicated in Table 5, the DD patient group included only 2 mitochondrial gene defects, SPG7 as part of hereditary spastic paraplegia's and MFN2 underlying a Charcot-Marie-Tooth disease phenotype. 
Table 4: Mitochondrial gene functions affected in MD patients. Disease causing nuclear genes, identified in patients with a primary mitochondrial disorder (MD patients), clustered according to their function in mitochondrial metabolism.

\begin{tabular}{|c|c|c|}
\hline gene & function & reference \\
\hline \multicolumn{3}{|c|}{ mitochondrial substrate import } \\
\hline SLC25A32 & mitochondrial folate transporter & Haitina et al., 2006 \\
\hline \multicolumn{3}{|c|}{ mitochondrial substrate metabolism } \\
\hline ACAD8 & Isobutyryl-CoA dehydrogenase & Nguyen et al., 2002 \\
\hline AMACR & Alpha-methylacyl-CoA racemase & Mubiru et al., 2004 \\
\hline \multicolumn{3}{|c|}{ mitochondrial protein metabolism; translation and degradation } \\
\hline AARS2 & alanyl-tRNA synthetase & Gotz et al., 2011 \\
\hline MTFMT (2x) & mitochondrial methionyl-tRNA formyltransferase & Takeuchi et al., 1998 \\
\hline MTO1 & tRNA modification and protein synthesis & Ghezzi et al., 2012 \\
\hline KARS & lysyl-tRNA synthetase & Targoff et al., 1993 \\
\hline QRSL1 & glu-tRNA synthetase & Nagao et al., 2009 \\
\hline TRMU & mitochondrial tRNA modification & Yan et al., 2005 \\
\hline C12ORF65 & release proteins from ribosomes & Antonicka et al., 2010 \\
\hline CLPP & mitochondrial proteolytic complex & Jenkinson et al., 2013 \\
\hline \multicolumn{3}{|c|}{ mitochondrial phospholipid metabolism } \\
\hline SERAC1 & phosphatidylglycerol remodeling & Wortmann et al., 2012 \\
\hline TAZ & cardiolipin remodeling & Acehan et al., 2011 \\
\hline \multicolumn{3}{|c|}{ ETC subunits, assembly factors, cofactors } \\
\hline NDUFA12 & complex I subunit & Triepels et al., 2000 \\
\hline NDUFS7 & complex I subunit & Visch et al., 2004 \\
\hline NDUFV2 & complex I subunit & de Coo et al., 1995 \\
\hline COQ7 & coenzyme $Q$ biosynthesis & Freyer et al., 2015 \\
\hline TMEM126B & complex I assembly factor & Andrews et al., 2013 \\
\hline ATPAF2 & complex $\mathrm{V}$ assembly factor & Wang et al. (2001) \\
\hline NDUFAF5 & complex I assembly factor & Sugiana et al., 2008 \\
\hline NDUFAF4 & complex I assembly factor & Karp et al., 2004 \\
\hline TMEM126A & complex I assembly factor & Wessels et al., 2013 \\
\hline \multicolumn{3}{|c|}{ mtDNA maintanance, replication and nucleotide metabolism } \\
\hline RRM2B & ribonucleotide reductase & Bourdon et al., 2007 \\
\hline$F B X L 4(2 x)$ & phosphorylation-dependent ubiquitination & Bonnen et al., 2013 \\
\hline DNA2 & mtDNA replication, mtDNA base-excision repair & Ronchi et al., 2013 \\
\hline PYCR2 & Pyrroline-5-carboxylate reductase & Mei-Ling Kuo et al., 2016 \\
\hline DHODH & pyrimidine biosynthesis & JingXian Fang et al., 2013 \\
\hline C10ORF2 & mtDNA helicase & Spelbrink et al., 2001 \\
\hline \multicolumn{3}{|c|}{ mitochondrial fusion and fision } \\
\hline SLC25A46 & interacts with mitochondrial fusion machinery & Janer et al., 2016 \\
\hline \multicolumn{3}{|c|}{ mitochondrial localization } \\
\hline C19orf12 & function unclear & Landoure et al., 2013 \\
\hline
\end{tabular}




\section{no mitochondrial localization or function reported}

\begin{tabular}{lll} 
SLC19A3 (2x) & transmembrane thiamine transporter & Vernau et al., 2015 \\
IER3IP1 & function unclear & Yiu et al., 2004 \\
SLC16A2 & transporter of thyroid hormones & Wrutniak-Cabello et al., 2001 \\
CHRNE & acetylcholine receptor subunit & Witzemann et al., 1996 \\
IARS & Isoleucyl-tRNA synthetase & Kopajtich et al., 2016 \\
\hline
\end{tabular}

Table 5: Mitochondrial gene functions affected in DD patients. Disease causing nuclear genes, identified in DD patients, classified according to their function in mitochondrial metabolism.

\begin{tabular}{|c|c|c|}
\hline gene & function & reference \\
\hline \multicolumn{3}{|c|}{ mitochondrial protein metabolism; degradation } \\
\hline SPG7 & part of the m-AAA metalloproteinase complex & Warnecke et al, 2007 \\
\hline \multicolumn{3}{|c|}{ mitochondrial fusion and fision } \\
\hline MFN2 & mitochondrial fusion & Santel et al., 2001 \\
\hline
\end{tabular}

\section{Discussion}

We used a next-generation sequencing strategy in a cohort of 84 patients with primary mitochondrial disease and 33 patients, where a mitochondrial cause is part of the differential diagnosis. In $69 \%$ of the patients we identified a disease causing genetic defect, of which $21 \%$ was solved by mtDNA sequencing and $49 \%$ by subsequent WES analysis. Compared to conventional Sanger sequencing methods, which only solved $11 \%(25)$, primarily due to limitations on the number of genes being sequenced, this is a major step forward. Selective sequencing of targeted gene panels in two studies, including 1598 and 1034 mitochondrial disease genes, resulted in respectively $22 \%$ (102 suspected mitochondrial patients) (26) and $24 \%$ (42 mitochondrial patients) (27) diagnostic yield. This is also much less than in our study, which can be explained by the incompleteness of the panels (more details below). In a previous next-generation sequencing-based study, which included 113 paediatric patients with suspected mitochondrial disease, screening both the mtDNA and the exome, resulted in a diagnostic yield of 59\% (28). Another study performing WES in 109 paediatric and young adult patients with suspected mitochondrial disease, established a genetic diagnosis in $39 \%$ of the patient cohort (after exclusion of mtDNA mutations) (21). Our paediatric patient group consisted of 91 patients, for which we could establish a genetic diagnosis in $67 \%$ (10 mtDNA and 51 nuclear gene mutations). Our higher yield is most likely caused by a complete analysis of both the mtDNA and the exome, a strong selection for genetic cases and the follow-up investigations in case of an unknown gene. In conclusion, our data shows that mtDNA sequencing followed by a complete whole exome analysis is the preferred strategy to identify the genetic basis of heterogeneous mitochondrial disease. 
As the mtDNA genome is relatively small, NGS allows cost effective and efficient testing of many patient samples within a single run, with sufficient sensitivity on blood DNA. Where traditionally several complementary methods had to be used for detection and quantification of mtDNA mutations (e.g. RFLP, ARMS-qPCR, sanger sequencing, MitoCHIP, Southern blot analysis), NGS can be used as a single method for identifying point mutations and indels, large deletion breakpoints and quantifying heteroplasmy levels of point mutations and small indels with high sensitivity and specificity (14). Only to accurately measure heteroplasmy levels of large mtDNA deletions and mtDNA depletion, additional qPCR based quantification is required. NGS of the mtDNA solved $21 \%$ of our patient cohort (all MD patients), mainly, but not only, consisting of patients with early disease onset (78\% paediatric patients). In the pure paediatric subgroup, primary mtDNA mutations accounted for $11 \%(10 / 91)$ of the genetic defects, which is in line with earlier reports that estimated involvement of a mtDNA defect in $<20 \%$ of the paediatric mitochondrial patients (29). In our adult subgroup, mtDNA defects accounted for $54 \%(14 / 26)$, which is lower than the previously estimated $70-75 \%$, possibly as a consequence of the relatively small number of adult patients in our cohort $(29,30)$. As shown in Table 1 , most mtDNA patients carried LHON and MIDD/MELAS causing mutations, which was also likely based on the clinical presentation. Both MIDD and MELAS symptoms were caused by the m.3243A>G mutation, in line with what has been reported before (31). Yet, as indicated by the heteroplasmy levels (24-41\% in MELAS and 7-40\% in MIDD), variation in symptom manifestations among these patients could not solely be explained from the differences in heteroplasmy level, making prognostic predictions challenging.

Using WES, we identified a disease causing variant in an additional $49 \%$ of the patient cohort, where we applied a variant selection strategy fitting the presumed genetic mode of disease inheritance. In a group with likely inherited disease (consanguineous and/or multiple patients), filtering for recessive mutations solved $69 \%$ of the patients. A group of single patients from non-consanguineous parents was also screened for heterozygous mutations in known (OMIM) AD disease genes, which resulted in an overall genetic diagnosis in $55 \%$ of the patients, of which $16 \%$ of the identified variants were de novo. To reduce the huge amount of potential dominant mutations, we have restricted our heterozygous variant selection to OMIM-reported dominant disease genes, and therefore might have missed some de novo cases. This could be overcome by WES trio-analysis. Additionally, the higher likelihood of an inherited genetic cause, the availability of homozygosity mapping data and of exome data of affected siblings might have contributed to a higher diagnostic yield in the first group. Our strategy did not result in large differences in diagnostic yield between mitochondrial patients (MD) and patients from the differential diagnostic procedure (DD). In an additional $7 \%$ of the WES cohort we found a genetic defect with a clear lead to the 
patient's phenotype (data not shown). For these cases, further laboratory testing or patient screening should reveal which genes can be classified as disease causing.

In $84 \%$ (31/37) of the MD patients, symptoms were caused by a gene with a reported mitochondrial function or localization. As expected, this was significantly lower in the DD patient group $(15 \%(3 / 20))$. Our results showed that in patients with primary mitochondrial disease, most genetic defects were associated with mitochondrial protein metabolism and OXPHOS function, where a majority of the OXPHOS defects were found in complex I genes. In line with previously reported complex I deficient patients, we found that defects in NDUFS7, NDUFA12 and NDUFAF5 caused Leigh-syndrome or a Leigh-like-phenotype (32). Although NDUFV2 defects have also been reported in association with Leigh syndrome (33), our patient exclusively expressed symptoms of white matter degeneration (encephalopathy). Also, we identified TMEM126A as an underlying cause of isolated complex I deficiency, therewith supporting co-migration studies that characterized TMEM126A as an early assembly factor of complex I (34). Patients with mtDNA abnormalities carried mutations in RRM2B, c10orf2 and DNA2, which were all functionally related to mtDNA maintenance, and presented as the third largest group. Interestingly, we also found the functionally uncharacterized c19orf12 gene associated with mtDNA depletion.

In 5 MD patients, we identified a causal gene for which no direct mitochondrial function or localization has been reported. This included 4 genes involved in different cellular processes (SLC19A3, SLC16A2, IARS, CHRNE). In two families with classical Leigh-syndrome we identified a defect in the thiamine transporter SLC19A3, where despite a decrease in mitochondrial oxygen consumption, complex activities seemed normal in muscle and skin tissue (35). Thiamine-diphosphate, the active form of thiamine, has been reported as an essential cofactor for several mitochondrial enzymes, including the pyruvate dehydrogenase complex (36). A second transmembrane transporter defect was found in the thyroid hormone (T3/T4) transporter SLC16A2, in a patient with Allan-Herndon Dudley syndrome, which resulted in a CII and CIV deficiency. Also here mitochondrial dysfunction seems to be a secondary effect of a transport deficiency, where triiodothyronine (T3) has been suggested as an important regulator of mitochondrial activity (37). Furthermore, we found a mutation in the cytoplasmic located aminoacyl-tRNA synthetase IARS to cause complex I deficiency in skin and muscle tissue of a patient with a multi-system disorder, and a defect in the acetylcholine receptor subunit epsilon CHRNE associated with CII deficiency, COX negative muscle fibres and abnormally shaped muscle mitochondria in CPEO with myopathy. In another patient, who was classified with MD based on the classical mitochondrial disease symptoms involved, the functionally uncharacterized IER3IP1 gene was identified. Although, no OXPHOS measurements were performed in this patient, knockdown of IER3IP1 was 
reported to affect mitochondrial function by decreasing the mitochondrial membrane potential and increasing cytochrome $\mathrm{C}$ release (38). The genetic defects in these patients are therefore important to further characterize the underlying processes and clarify their role in mitochondrial disease.

$30 \%$ of the disease-causing genes identified in our MD-patients were not included in the MitoCarta database at the moment of identification, and could therefore be missed with panel-based sequencing approaches. In addition to the 5 genes described above, this involved several genes with a reported function in mitochondrial metabolism, indicating that it can be difficult to keep databases or panels up to date with recent discoveries. Also, the contribution of non-mitochondrial disease genes to complex multi-genic mitochondrial disease might be missed when applying targeted sequencing methods, as illustrated by two of the mitochondrial patient cases, in which defects in more than a single gene were involved. Our data shows that WES is the most comprehensive and unbiased approach to establish a genetic diagnosis in mitochondrial disease, able to fully resolve complex multigenic disease manifestations. To avoid incidental findings in a diagnostic setting one might consider pre-screening WES data with virtual gene panels; however, in heterogeneous disorders, this can easily result in inefficient and sequential use of different virtual gene panels, and still miss the complete picture in multi-genic disorders. Obviously, a disadvantage of WES in diagnostics settings will be the identification of novel, non-reported gene defects that often require extensive experimental setups to validate a possible role in mitochondrial disease. Embedding within or contact with a specialized research group should therefore be preferable. Identification of the genetic defect is not only important for diagnostics, but also for therapeutic interventions. Patients with SLC25A32, SLC19A3 and TMEM126B defects showed improvement upon treatment with respectively riboflavin, biotin/thiamine and high fat-diet $(35,39,40)$. Yet, despite the significant improvements in the genetic diagnosis of mitochondrial disorders, the development of novel therapies is lagging behind.

\section{Acknowledgement}

This work was supported by the Princes Beatrix Spierfonds (grant no: W.OR11-24) and Stichting MetaKids. Funding sources had no involvement in study design; collection, analysis or interpretation of data; writing of the manuscript; or the decision to submit the article for publication. 


\section{References}

1. Rotig A, Munnich A. Genetic features of mitochondrial respiratory chain disorders. J Am Soc Nephrol. 2003;14(12):2995-3007.

2. McFarland R, Taylor RW, Turnbull DM. A neurological perspective on mitochondrial disease. Lancet Neurol. 2010;9(8):829-40.

3. Hellebrekers DM, Wolfe R, Hendrickx AT, de Coo IF, de Die CE, Geraedts JP, et al. PGD and heteroplasmic mitochondrial DNA point mutations: a systematic review estimating the chance of healthy offspring. Hum Reprod Update. 2012;18(4):341-9.

4. Thorburn DR, Dahl HH. Mitochondrial disorders: genetics, counseling, prenatal diagnosis and reproductive options. Am J Med Genet. 2001;106(1):102-14.

5. Calvo SE, Mootha VK. The mitochondrial proteome and human disease. Annu Rev Genomics Hum Genet. 2010;11:25-44.

6. Prokisch $\mathrm{H}$, Ahting $\mathrm{U}$. MitoP2, an integrated database for mitochondrial proteins. Methods $\mathrm{Mol}$ Biol. 2007;372:573-86.

7. Alston CL, Rocha MC, Lax NZ, Turnbull DM, Taylor RW. The genetics and pathology of mitochondrial disease. J Pathol. 2017;241(2):236-50.

8. Hui J, Kirby DM, Thorburn DR, Boneh A. Decreased activities of mitochondrial respiratory chain complexes in non-mitochondrial respiratory chain diseases. Dev Med Child Neurol. 2006;48(2):132-6.

9. Fan H, Civalier C, Booker JK, Gulley ML, Prior TW, Farber RA. Detection of common diseasecausing mutations in mitochondrial DNA (mitochondrial encephalomyopathy, lactic acidosis with stroke-like episodes MTTL1 $3243 \mathrm{~A}>\mathrm{G}$ and myoclonic epilepsy associated with ragged-red fibers MTTK 8344A>G) by real-time polymerase chain reaction. J Mol Diagn. 2006;8(2):277-81.

10. Wang J, Venegas V, Li F, Wong LJ. Analysis of mitochondrial DNA point mutation heteroplasmy by ARMS quantitative PCR. Curr Protoc Hum Genet. 2011;Chapter 19:Unit 196.

11. Finsterer J, Harbo HF, Baets J, Van Broeckhoven C, Di Donato S, Fontaine B, et al. EFNS guidelines on the molecular diagnosis of mitochondrial disorders. Eur J Neurol. 2009;16(12):1255-64.

12. van Eijsden RG, Gerards M, Eijssen LM, Hendrickx AT, Jongbloed RJ, Wokke JH, et al. Chip-based mtDNA mutation screening enables fast and reliable genetic diagnosis of OXPHOS patients. Genet Med. 2006;8(10):620-7.

13. McCormick E, Place E, Falk MJ. Molecular genetic testing for mitochondrial disease: from one generation to the next. Neurotherapeutics. 2013;10(2):251-61.

14. Tang S, Huang T. Characterization of mitochondrial DNA heteroplasmy using a parallel sequencing system. Biotechniques. 2010;48(4):287-96.

15. Xie HM, Perin JC, Schurr TG, Dulik MC, Zhadanov SI, Baur JA, et al. Mitochondrial genome sequence analysis: a custom bioinformatics pipeline substantially improves Affymetrix MitoChip v2.0 call rate and accuracy. BMC Bioinformatics. 2011;12:402.

16. Wong LJ, Perng CL, Hsu CH, Bai RK, Schelley S, Vladutiu GD, et al. Compensatory amplification of mtDNA in a patient with a novel deletion/duplication and high mutant load. J Med Genet. 2003;40(11):e125.

17. He L, Chinnery PF, Durham SE, Blakely EL, Wardell TM, Borthwick GM, et al. Detection and quantification of mitochondrial DNA deletions in individual cells by real-time PCR. Nucleic Acids Res. 2002;30(14):e68.

18. Cui H, Li F, Chen D, Wang G, Truong CK, Enns GM, et al. Comprehensive next-generation sequence analyses of the entire mitochondrial genome reveal new insights into the molecular diagnosis of mitochondrial DNA disorders. Genet Med. 2013;15(5):388-94. 
19. Huang T. Next generation sequencing to characterize mitochondrial genomic DNA heteroplasmy. Curr Protoc Hum Genet. 2011; Chapter 19:Unit19 8.

20. Li M, Schonberg A, Schaefer M, Schroeder R, Nasidze I, Stoneking M. Detecting heteroplasmy from high-throughput sequencing of complete human mitochondrial DNA genomes. Am J Hum Genet. 2010;87(2):237-49.

21. Wortmann SB, Koolen DA, Smeitink JA, van den Heuvel L, Rodenburg RJ. Whole exome sequencing of suspected mitochondrial patients in clinical practice. J Inherit Metab Dis. 2015;38(3):437-43.

22. Li H, Durbin R. Fast and accurate short read alignment with Burrows-Wheeler transform. Bioinformatics. 2009;25(14):1754-60.

23. Li H, Handsaker B, Wysoker A, Fennell T, Ruan J, Homer N, et al. The Sequence Alignment/Map format and SAMtools. Bioinformatics. 2009;25(16):2078-9.

24. Seelow D, Schuelke M, Hildebrandt F, Nurnberg P. HomozygosityMapper--an interactive approach to homozygosity mapping. Nucleic Acids Res. 2009;37(Web Server issue):W593-9.

25. Neveling K, Feenstra I, Gilissen C, Hoefsloot LH, Kamsteeg EJ, Mensenkamp AR, et al. A posthoc comparison of the utility of sanger sequencing and exome sequencing for the diagnosis of heterogeneous diseases. Hum Mutat. 2013;34(12):1721-6.

26. Lieber DS, Calvo SE, Shanahan K, Slate NG, Liu S, Hershman SG, et al. Targeted exome sequencing of suspected mitochondrial disorders. Neurology. 2013;80(19):1762-70.

27. Calvo SE, Compton AG, Hershman SG, Lim SC, Lieber DS, Tucker EJ, et al. Molecular diagnosis of infantile mitochondrial disease with targeted next-generation sequencing. Sci Transl Med. 2012;4(118):118ra10.

28. Pronicka E, Piekutowska-Abramczuk D, Ciara E, Trubicka J, Rokicki D, Karkucinska-Wieckowska $A$, et al. New perspective in diagnostics of mitochondrial disorders: two years' experience with whole-exome sequencing at a national paediatric centre. J Transl Med. 2016;14(1):174.

29. Schaefer AM, McFarland R, Blakely EL, He L, Whittaker RG, Taylor RW, et al. Prevalence of mitochondrial DNA disease in adults. Ann Neurol. 2008;63(1):35-9.

30. Gorman GS, Schaefer AM, Ng Y, Gomez N, Blakely EL, Alston CL, et al. Prevalence of nuclear and mitochondrial DNA mutations related to adult mitochondrial disease. Ann Neurol. 2015;77(5):753-9.

31. de Wit HM, Westeneng HJ, van Engelen BG, Mudde AH. MIDD or MELAS : that's not the question MIDD evolving into MELAS : a severe phenotype of the m.3243A $>\mathrm{G}$ mutation due to paternal coinheritance of type 2 diabetes and a high heteroplasmy level. Neth J Med. 2012;70(10):460-2.

32. Rahman S, Thorburn D. Nuclear Gene-Encoded Leigh Syndrome Overview. In: Pagon RA, Adam MP, Ardinger HH, Wallace SE, Amemiya A, Bean LJH, et al., editors. GeneReviews(R). Seattle WA: University of Washington, Seattle. GeneReviews is a registered trademark of the University of Washington, Seattle; 1993.

33. Cameron JM, MacKay N, Feigenbaum A, Tarnopolsky M, Blaser S, Robinson BH, et al. Exome sequencing identifies complex I NDUFV2 mutations as a novel cause of Leigh syndrome. Eur J Paediatr Neurol. 2015;19(5):525-32.

34. Wessels HJ, Vogel RO, Lightowlers RN, Spelbrink JN, Rodenburg RJ, van den Heuvel LP, et al. Analysis of 953 human proteins from a mitochondrial HEK293 fraction by complexome profiling. PLoS One. 2013;8(7):e68340.

35. Gerards M, Kamps R, van Oevelen J, Boesten I, Jongen E, de Koning B, et al. Exome sequencing reveals a novel Moroccan founder mutation in SLC19A3 as a new cause of early-childhood fatal Leigh syndrome. Brain. 2013;136(Pt 3):882-90.

36. Ortigoza-Escobar JD, Serrano M, Molero M, Oyarzabal A, Rebollo M, Muchart J, et al. Thiamine transporter-2 deficiency: outcome and treatment monitoring. Orphanet J Rare Dis. 2014;9:92.

37. Wrutniak-Cabello C, Casas F, Cabello G. Thyroid hormone action in mitochondria. J Mol Endocrinol. 2001;26(1):67-77. 
38. Sun J, Ren D. IER3IP1 deficiency leads to increased beta-cell death and decreased beta-cell proliferation. Oncotarget. 2017;8(34):56768-79.

39. Hellebrekers D, Sallevelt S, Theunissen TEJ, Hendrickx ATM, Gottschalk RW, Hoeijmakers JGJ, et al. Novel SLC25A32 mutation in a patient with a severe neuromuscular phenotype. European journal of human genetics : EJHG. 2017;25(7):886-8.

40. Theunissen TEJ, Gerards M, Hellebrekers D, van Tienen FH, Kamps R, Sallevelt S, et al. Selection and Characterization of Palmitic Acid Responsive Patients with an OXPHOS Complex I Defect. Frontiers in molecular neuroscience. 2017;10:336. 



\section{CHAPTER 3}

Rapid resolution of blended or composite multigenic disease in infants by whole exome sequencing

Tom E.J. Theunissen*, Suzanne C.E.H. Sallevelt*, Debby M.E.I. Hellebrekers, Bart de Koning, Alexandra T.M. Hendrickx, Bianca J.C. van den Bosch, Rick Kamps, Kees Schoonderwoerd, Radek Szklarczyk, Elvira N.M. Mulder-Den Hartog, Irenaeus F.M. de Coo, Hubert J.M. Smeets.

*=These authors contributed equally

The Journal of pediatrics. 2017;182:371-4.e2. doi: 10.1016/j.jpeds.2016.12.032 


\begin{abstract}
Whole-exome sequencing (WES) identified multiple genetic causes in two infants with heterogeneous disease. Three gene defects in the first patient explained all symptoms, but manifestations were overlapping (blended phenotype). Two gene defects in the second patient explained non-overlapping symptoms (composite phenotype). WES rapidly and comprehensively resolves heterogeneous genetic disease.
\end{abstract}




\section{Introduction}

A variety of disease manifestations in young infants, explainable by combinations of genetic and environmental factors, can hamper an early and complete genetic diagnosis. Whereas targeted analysis of candidate genes might not resolve the complete clinical picture, WES is unbiased, allowing identification and evaluation of the majority of the patient's genetic variants. In clinical practice, WES has become the prime test for genetically heterogeneous monogenic disorders, with no straightforward genotype-phenotype correlation and with unknown genes involved. WES also seems preferable to decipher complex disease, which could be caused by genes with overlapping (blended) or discrete (composite) manifestations $(1,2)$, in young children at an early stage of disease manifestation. This could especially apply to affected children from consanguineous marriages, where the parents potentially share carrier-ship of more genetic diseases than outbred marriages.

\section{Material and Methods}

\section{Ethical approval and informed consent}

Procedures with human participants were in accordance with the ethical standards of the local medical ethical committee (MUMC+) and the 1964 Helsinki declaration. Written informed consent was obtained from the parents of the pediatric patients.

\section{Patients}

Patient 1 , the second child of healthy consanguineous Moroccan parents (first-degree cousins), was hospitalized with heterogeneous neurological symptoms from birth on. Her sister was more mildly affected and showed only part of the symptom manifestations. Clinical symptoms of both siblings are depicted in Figure $1 \mathrm{~A}$ (clinical details in Table S1). Patient 2, a child of healthy non-consanguineous parents, was born with a multisystemic disease. Clinical manifestations are shown in Figure 1B (clinical details in Table S2). 
A

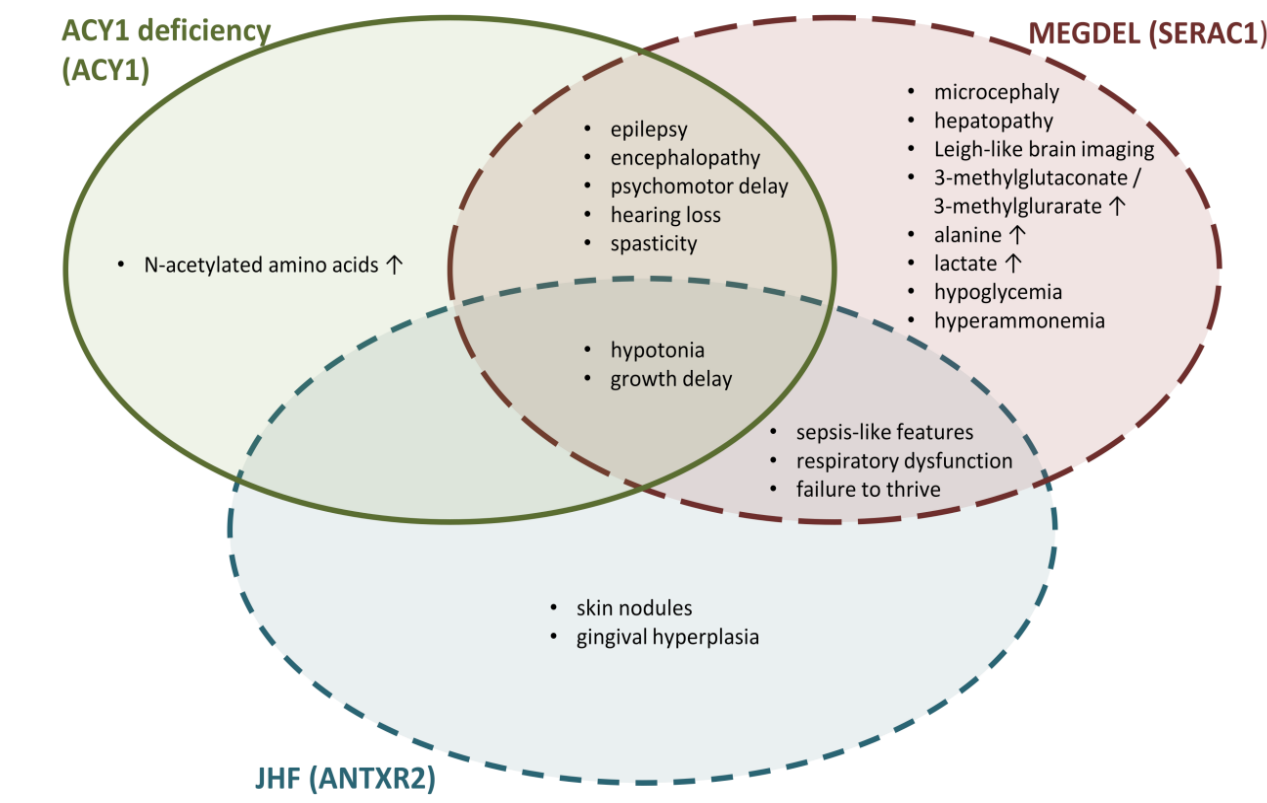

B

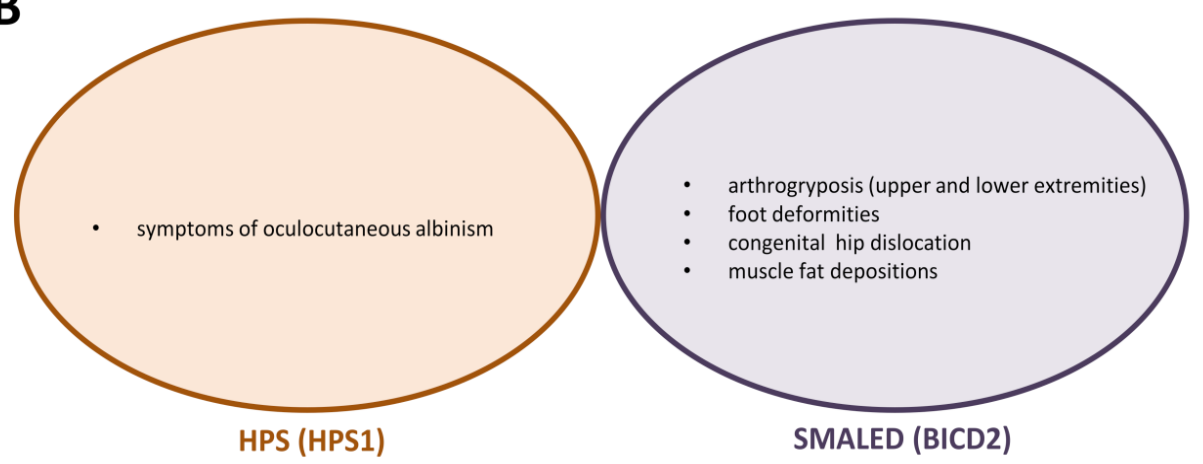

Figure 1: Categorization of observed clinical features according to the individual gene defects. (A) Patient 1 showed a blended phenotype as large overlap in neurological features was found, especially between ACY1 and SERAC1 symptoms. The sister was solely affected by JHF symptoms (ANTXR2). (B) Patient 2 had a composite phenotype due to genetic defects in HPS1 and BICD2.

\section{Homozygosity mapping and WES}

Homozygosity mapping was performed by Affymetrix Human Mapping 250K array and Genotyping console 4.0. Homozygosity regions $(\mathrm{ROH})$ were defined by "Homozygosity mapper", using a cutoff of 5MB. Exons were captured using Agilent SureSelect version 4, including UTRs, exome enrichment kit. Sequencing was performed on an Illumina HiSeq2000 
platform, using a 2x100 bp paired end setting. Bcl2fastq 1.8.4 (Illumina) allowed Basecalling and demultiplexing, and BWA 0.5.9 was used for read alignment against human reference genome hg19. Duplicate reads were removed by Picard software suite 1.77 (Broadinstitute) and variant calling was performed with GATK 2.1-8 (Broadinstitute). Exome data of patient 1 were filtered for homozygous variants in $\mathrm{ROH}^{\prime}$ s. Variants with allele frequencies lower than 1\% (dbSNP137, ExAC), consisting of non-synonymous substitutions, INDELs, nonsense mutations and splice-variants were evaluated. Non-annotated variants were maintained, unless allele frequencies exceeded $5 \%$ prevalence in our in house patient database. Pathogenicity of non-synonymous missense mutation was estimated by Polymorphism Phenotyping-2 (PolyPhen-2), Sorting Intolerant From Tolerant (SIFT), PROVEAN and Mutation Taster. Missense mutations were considered pathogenic if predicted damaging by at least two in silico prediction-tools. Nonsense, frameshift and splice-site variations were maintained. WES data of patient 2 was filtered for compound heterozygous, homozygous and dominant de novo mutations, using a comparable strategy. Potential dominant de novo mutations were limited to OMIM reported disease-genes with known dominant mutations.

\section{Results}

\section{Patient 1: SERAC1, ACY1 and ANTXR2 mutations}

A homozygous in-frame insertion was identified in SERAC1 (NC_000006.12, NM_032861.3:c.1349_1350insATC), resulting in an additional serine residue (p.Ser450) in exon 13, which has not been previously reported in patients or SNP databases. It is highly pathogenic according to Provean (-9.592) and MutationTaster (disease causing). The serine residue located at the site of insertion is perfectly conserved in all vertebrates. Sanger sequencing confirmed a recessive segregation pattern within the family. The sister did not carry this variant.

The ANTXR2 gene contained a homozygous missense mutation (NG_015987.1, NM_001145794.1:c.1142A>G). The p.(Tyr381Cys) substitution affects an evolutionary conserved amino acid position and was previously reported as a disease causing mutation (HGMD: CM033747) (3). The parents were heterozygous and both the index patient and sister were homozygous for the mutation.

Athird homozygous mutation wasidentified inACY1(NG_012036.1,NM_000666.2:c.811G>A), changing a non-polar alanine to polar threonine ( $p$.(Ala271Thr)) in a conserved M20 dimerization domain (Pfam07687). The variant has an allele frequency of $\sim 0.0017 \%$ (ExAC 
database). Polyphen2 (0.979, probably damaging) and Mutation taster (disease causing) classified the mutation as pathogenic (the variant, rs765511074, is of uncertain significance according to Clinvar). Segregation testing revealed that both parents and the sister were heterozygous for the mutation.

\section{Patient 2: HPS1 and BICD2 mutations}

Patient 2 was compound heterozygous for two HPS1 mutations, p.(Arg173*) and p.(GIn397Serfs*2) (NG_009646.1, NM_000195.4:c.[517C>T];[1189delC]). The nonsense mutation causes an early stop codon, implying a loss of 527 amino acids or, more likely, nonsense-mediated decay of the mRNA. p.(Arg173*) has not been reported in patients or annotated in SNP-databases. The p.(GIn397Serfs*2) mutation results in a frameshift with an early stop codon, most likely causing nonsense-mediated mRNA decay. The mutation has been reported as disease causing (HGMD database: CD982692) (4). Both parents were heterozygous mutation carriers.

A second gene defect was located in BICD2 (NG_033908.1, NM_001003800.1:c.539A>C). The dominant missense mutation affects a conserved amino acid position ( $p$.(Asp180Ala)) and has highly damaging properties according to MutationTaster (disease causing), PROVEAN (-7.6), SIFT (deleterious: 0.03) and PolyPhen2 (0.980, probably damaging). Several pathogenic heterozygous $B I C D 2$ mutations have been reported (both autosomal dominant and de novo), however this specific mutation has not been previously reported in patients or SNP-databases. Sanger sequencing and non-paternity testing validated both parents as non-carriers, confirming a de novo introduction of the dominant BICD2 mutation.

\section{Discussion}

\section{Gene-phenotype relations}

Clinical symptoms of patient 1 were compared with other patients, having mutations in these three genes (Table S1). Similar to the SERAC1 cases, patient 1 showed Leigh syndrome-related disease manifestations, with characteristic MRI and neurological and muscular impairments (5). Involvement of 3-methylglutaconic aciduria with sensorineural deafness and encephalopathy is typically explained by SERAC1 mutations, underlying MEGDEL syndrome (3-methylglutaconic aciduria, deafness, encephalopathy, and Leigh-like disease). Most of the MEGDEL reported symptoms were present in patient 1 . ANTXR2 gene defects underlie Juvenile hyaline fibromatosis (JHF) or infantile systemic hyalinosis (ISH), 
with clinical similarity involving subcutaneous skin nodules, gingival hypertrophy and joint contractures. Similar to two reported Moroccan siblings with JHF(3), both affected siblings were homozygous for the p.(Tyr381Cys) mutation. ACY1 mutations have been reported to cause aminoacylase 1 deficiency. Urinary metabolite screening of patient 1 provided a specific profile of accumulating $\mathrm{N}$-acetylated amino acids, characteristic for ACY1 deficiency. Comparable to other $A C Y 1$ cases, patient 1 suffered from pronounced neurological symptoms with hypotonia, spasticity and growth delay $(6,7)$. Although several patient reports highlight a role for aminoacylase deficiency in disease manifestation (6), the clinical significance of the enzyme deficiency has also been questioned $(8,9)$.

Symptoms deriving from the three individual gene defects showed overlap in patient 1 (Figure $1 \mathrm{~A}$, a blended phenotype). Since all neurological features could be explained from the SERAC1 defect, it is uncertain whether ACY1 deficiency contributed to disease manifestation or its severity. Yet, based on the available data, a possible role cannot be excluded. If parents would opt for prenatal diagnosis (PND) in a subsequent pregnancy, ACY1 cannot be ignored, having implications for the number of gene defects tested. Discussions on the clinical impact of a genetic defect can cause dilemmas in a PND-context, as is the case for JHF, in which symptom manifestation might be relatively mild. Preimplantation genetic diagnosis (PGD) and selecting mutation-free embryos can be a good alternative, but selection for three or more diseases requires a trophectoderm biopsy, or new single cell analysis methods for blastomeres. Anyhow, an increase in the number of conditions tested reduces the number of embryos suitable for transfer.

In patient 2, the HPS1 mutation could explain the oculocutaneous albinism as a characterteristic feature of Hermansky-Pudlak syndrome type 1 (10). No indications were found for other reported HPS1 symptoms (Table S2). A second gene defect was found in $B I C D 2$, in which several autosomal dominant mutations have been reported in patients with Spinal Muscular Atrophy, Lower Extremity-preDominant type2 (SMALED2). SMALED2 is a dominant spinal muscular atrophy with congenital onset and a variety of symptoms, mainly causing weakness of the lower limb muscles and affecting joint function (contractures) of the lower extremities (11). For some patients involvement of the upper extremities has been reported as well. Symptoms of patient 2 overlapped with previously reported BICD2 symptoms (Table S2). p.(Asp180Ala) is located in the same domain as a SMALED2 mutation reported to cause Golgi-apparatus fragmentation (12), and therefore likely explains the remaining symptoms in patient 2 , having a composite phenotype (Figure $1 \mathrm{~B}$ ). 


\section{WES in multigenic disease}

Our data shows the utility of WES in the characterization of clinically complex phenotypes which can be explained from multiple gene defects. Whereas targeted gene analysis limits to single or small panels of candidate genes, WES is unbiased and comprehensive, as it identifies almost all genetic variants in the exons and flanking introns. This latter is crucial for establishing a genetic diagnosis in patients with heterogeneous disease manifestations, as symptoms might be explained by more than a single defect. Our patients were affected by multiple, Mendelian inherited, full penetrant gene defects which could individually explain a complex combination of disease manifestations. Each gene defect has been reported separately responsible for part of the clinical manifestations, as indicated in Figure 1. Based on the gene defects identified, clinical symptoms can be grouped into overlapping (blended) or discrete (composite) phenotypes, which can guide further clinical investigations. The latter is important, as identical symptoms might be explained by two independent gene defects. Functional follow-up investigations can discriminate their individual contribution and avoid a potential misclassification of benign WES variants as pathogenic (false positive variants). If parents would opt for prenatal diagnosis, to get an unaffected child, it should be clear what the consequences of the individual gene defects are, either in isolation or in combination with other gene defects. This dilemma is illustrated by the first family, where the contribution of the ACY1 mutation to the phenotype is currently unclear, and where it is shown that inheritance of the ANTXR2 mutation as a single gene defect can still result in good quality of life (based on the affected sib who reached adolescence with only mild symptoms). Whereas in clinical practice a genetic diagnosis is often completed once a single gene defect has been identified, our data illustrate the importance of pursuing genetic analysis until each individual symptom can be explained. WES is a comprehensive approach to establish an early and complete characterization of blended or composite multigenic disease, thereby enabling precision medicine and personalized care for young children with unique combined disease manifestations. WES will become the first, rapid test for children with heterogenic or multi-genetic disease, which would otherwise require time-consuming and expensive clinical investigations. This especially applies to patients from consanguineous marriages (DNA confirmed), where parents potentially share carrier-ship of more genetic diseases than outbred marriages and identity by descent (IBD) segments occur at higher rates than in children from out-bred parents. 


\section{References}

1. O'Donnell-Luria AH, Miller DT. A Clinician's perspective on clinical exome sequencing. Hum Genet. 2016;135(6):643-54.

2. Yang $Y$, Muzny DM, Xia F, Niu Z, Person R, Ding $Y$, et al. Molecular findings among patients referred for clinical whole-exome sequencing. JAMA. 2014;312(18):1870-9.

3. Hanks S, Adams S, Douglas J, Arbour L, Atherton DJ, Balci S, et al. Mutations in the gene encoding capillary morphogenesis protein 2 cause juvenile hyaline fibromatosis and infantile systemic hyalinosis. Am J Hum Genet. 2003;73(4):791-800.

4. Oh J, Ho L, Ala-Mello S, Amato D, Armstrong L, Bellucci S, et al. Mutation analysis of patients with Hermansky-Pudlak syndrome: a frameshift hot spot in the HPS gene and apparent locus heterogeneity. Am J Hum Genet. 1998;62(3):593-8.

5. Sarig O, Goldsher D, Nousbeck J, Fuchs-Telem D, Cohen-Katsenelson K, lancu TC, et al. Infantile mitochondrial hepatopathy is a cardinal feature of MEGDEL syndrome (3-methylglutaconic aciduria type IV with sensorineural deafness, encephalopathy and Leigh-like syndrome) caused by novel mutations in SERAC1. Am J Med Genet A. 2013;161A(9):2204-15.

6. Alessandri MG, Casarano M, Pezzini I, Doccini S, Nesti C, Cioni G, et al. Isolated mild intellectual disability expands the aminoacylase 1 phenotype spectrum. JIMD Rep. 2014;16:81-7.

7. Sass JO, Olbrich H, Mohr V, Hart C, Woldseth B, Krywawych S, et al. Neurological findings in aminoacylase 1 deficiency. Neurology. 2007;68(24):2151-3.

8. Sass JO, Mohr V, Olbrich H, Engelke U, Horvath J, Fliegauf M, et al. Mutations in ACY1, the gene encoding aminoacylase 1, cause a novel inborn error of metabolism. Am J Hum Genet. 2006;78(3):401-9.

9. Van Coster RN, Gerlo EA, Giardina TG, Engelke UF, Smet JE, De Praeter CM, et al. Aminoacylase I deficiency: a novel inborn error of metabolism. Biochem Biophys Res Commun. 2005;338(3):13226.

10. Gahl WA, Brantly M, Kaiser-Kupfer MI, Iwata F, Hazelwood S, Shotelersuk V, et al. Genetic defects and clinical characteristics of patients with a form of oculocutaneous albinism (HermanskyPudlak syndrome). N Engl J Med. 1998;338(18):1258-64.

11. Rossor AM, Oates EC, Salter HK, Liu Y, Murphy SM, Schule R, et al. Phenotypic and molecular insights into spinal muscular atrophy due to mutations in BICD2. Brain. 2015;138(Pt 2):293-310.

12. Neveling K, Martinez-Carrera LA, Holker I, Heister A, Verrips A, Hosseini-Barkooie SM, et al. Mutations in BICD2, which encodes a golgin and important motor adaptor, cause congenital autosomal-dominant spinal muscular atrophy. Am J Hum Genet. 2013;92(6):946-54. 


\section{Supplementary}

Table S1: Symptoms caused by SERAC1 (NM_032861.3:c.1349_1350insATC), ACY1 (NM_000666.2:c.811G>A) and ANTXR2 (NM_001145794.1:c.1142A>G) mutations.

\begin{tabular}{|c|c|c|c|}
\hline & & patient 1 (index) & sister \\
\hline \multirow{6}{*}{ 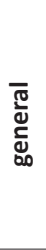 } & & Patient description & \\
\hline & genetic variants & $\begin{array}{l}\text { SERAC1:p.450insS, ACY1:p.(S271T), } \\
\text { ANTRX2:p.(Y381C) }\end{array}$ & ANTRX2:p.(Y381C) \\
\hline & alive (age) & no (died at 2 yrs) & yes (age 8 yrs) \\
\hline & first symptoms (age) & birth & 1 year \\
\hline & length and weight & $-3 S D$ & n.d. \\
\hline & birthweight & $2,300 \mathrm{~g}(-2 \mathrm{SD})$ & n.d. \\
\hline \multirow{33}{*}{ 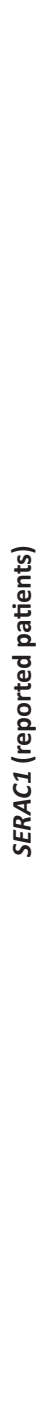 } & & Clinical findings & \\
\hline & sepsis-like features & yes & \\
\hline & feeding problems & yes & \\
\hline & failure to thrive / growth delay & yes & \\
\hline & microcephaly (1 patient) & borderline (-2 SD) & \\
\hline & hypotonia & yes & \\
\hline & psychomotor / developmental delay & yes & \\
\hline & developmental regression & yes & \\
\hline & absent speech & $\begin{array}{l}\text { yes; elementary speech development } \\
\text { disappeared }\end{array}$ & \\
\hline & epilepsy & yes & \\
\hline & encephalopathy & yes & \\
\hline & Leigh-like brain imaging (MRI/MRS) & yes & \\
\hline & dystonia / spacticity & yes & \\
\hline & sensorineural hearing loss & yes & \\
\hline & optic atrophy (bilateral) (1 patient) & $\begin{array}{l}\text { no; however, bilateral hyperopia (+6 dpt) } \\
\text { was diagnosed }\end{array}$ & \\
\hline & hepatopathy & yes & \\
\hline & respiratory dysfunction & yes & \\
\hline & & Biochemical data & \\
\hline & hypoglycemia (neonatal) & yes & \\
\hline & hyperammonemia (neonatal) & yes & \\
\hline & increased 3-methylglutaconate in urine & yes & \\
\hline & increased 3-methylglurarate in urine & yes & \\
\hline & increased alanine & yes (slightly) & \\
\hline & increased lactate in blood & yes & \\
\hline & $\begin{array}{l}\text { accumulation of free intracellular } \\
\text { cholesterol in fibroblasts }\end{array}$ & n.d. & \\
\hline & $\begin{array}{l}\text { increased phosphatidylglycerol-34:1 } \\
\text { to phosphatidylglycerol-36:1 ratio in } \\
\text { fibroblasts }\end{array}$ & n.d. & \\
\hline & decreased blood cholesterol levels & no & \\
\hline & $\begin{array}{l}\text { increased concentrations of specific } \\
\text { cardiolipin species in fibroblasts }\end{array}$ & n.d. & \\
\hline & \multicolumn{3}{|c|}{ Mitochondrial respiratory chain activities } \\
\hline & muscle tissue & n.d. & n.d. \\
\hline & fibroblasts & no abnormalities & \\
\hline & & Enzymatic activities & \\
\hline & 3-methylglutaconyl-CoA hydratase & n.d. & \\
\hline
\end{tabular}




\begin{tabular}{|c|c|c|c|}
\hline \multirow{14}{*}{ 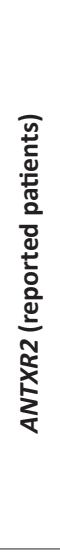 } & & Clinical findings & \\
\hline & skin nodules and/or papules & & yes \\
\hline & subcutaneous tumors & & \\
\hline & hyaline deposition in dermis & & \\
\hline & gingival hypertrophy & yes & yes \\
\hline & progressive joint contractures & & \\
\hline & bone deformities & & \\
\hline & persistent diarrhea & & \\
\hline & sepsis-like features & yes & \\
\hline & failure to thrive & yes & \\
\hline & growth delay & yes & \\
\hline & visceral involvement & & \\
\hline & coarse facial features & & \\
\hline & respiratory distress & yes & \\
\hline \multirow{11}{*}{ 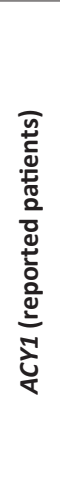 } & \multicolumn{3}{|c|}{ Clinical findings } \\
\hline & encephalopathy & yes & \\
\hline & autistic features & & \\
\hline & epilepsy & yes & \\
\hline & sensorineural hearing loss & yes & \\
\hline & growth delay & yes & \\
\hline & psychomotor / developmental delay & yes & \\
\hline & opisthotonic spasms (1 patient) & no; only generalized spasticity involved & \\
\hline & hypotonia & yes & \\
\hline & \multicolumn{3}{|c|}{ Biochemical data } \\
\hline & $\begin{array}{l}\text { increased } \mathrm{N} \text {-acetylated amino acid } \\
\text { levels (urine); indicating amino-acylase } 1 \\
\text { deficiency }\end{array}$ & yes & \\
\hline
\end{tabular}


Table S2: Symptoms caused by HPS1 (NM_000195.4:c.[517C>T];[1189delC]) and BICD2 (NM_001003800.1:c.539A>C) mutations.

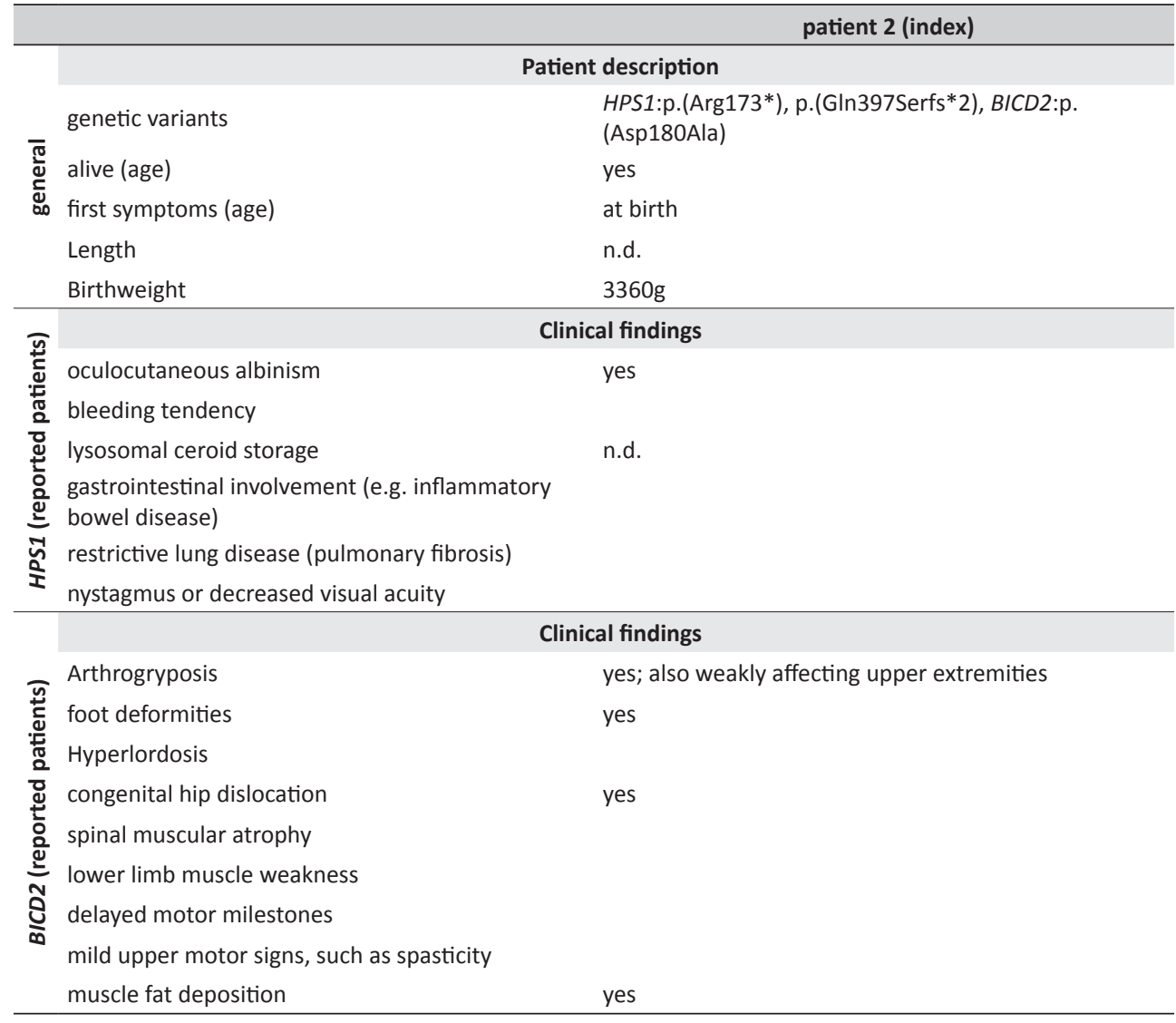




\title{
CHAPTER 4
}

\section{MRI abnormalities reveal severe Perrault syndrome}

\author{
due to CLPP defects
}

Tom E.J. Theunissen, Radek Szklarczyk, Mike Gerards, Debby M. E. I. Hellebrekers, Elvira N. M. Mulder-Den Hartog, Jo Vanoevelen, Rick Kamps, Bart de Koning, S. Lane Rutledge, Thomas Schmitt-Mechelke, Carola G. M. van Berkel, Marjo S. van der Knaap*, Irenaeus F. M. de Coo*, Hubert J. M. Smeets*

*= Joint senior authors

Frontiers in neurology. 2016;7:203. doi: 10.3389/fneur.2016.00203 


\begin{abstract}
In establishing a genetic diagnosis in heterogeneous neurological disease, clinical characterization and whole exome sequencing (WES) go hand-in-hand. Clinical data are essential, not only to guide WES variant selection and define the clinical severity of a genetic defect, but also to identify other patients with defects in the same gene. In an infant patient with sensorineural hearing loss, psychomotor retardation and epilepsy, WES resulted in identification of a novel homozygous CLPP frameshift mutation (c.21delA). Based on the gene defect and clinical symptoms, the diagnosis Perrault syndrome type 3 (PRLTS3) was established. The patient's brain-MRI revealed specific abnormalities of the subcortical and deep cerebral white matter and the middle blade of the corpus callosum, which was used to identify similar patients in the Amsterdam brain-MRI database, containing over 3000 unclassified leukoencephalopathy cases. In three unrelated patients with similar MRI abnormalities the CLPP gene was sequenced, and in two of them novel missense mutations were identified together with a large deletion that covered part of the CLPP gene on the other allele. The severe neurological and MRI-abnormalities in these young patients were due to the drastic impact of the CLPP mutations, correlating with the variation in clinical manifestations among previously reported patients. Our data shows that similarity in brainMRI patterns can be used to identify novel PRLTS3 patients, especially during early disease stages, when only part of the disease manifestations are present. This seems especially applicable to the severely affected cases in which CLPP function is drastically affected and MRI abnormalities are pronounced.
\end{abstract}




\section{Introduction}

For identification of the genetic causes of clinically and genetically heterogeneous neurological disease, whole exome sequencing (WES) has become the prime method of investigation as a rapid, single test. Especially for conditions that can be caused by a large number of candidate genes, WES provides an unbiased approach to identify the genetic cause, precluding comprehensive analysis of all individual genes (1-4). By contrast, in some neurological conditions, clinical symptoms or additional investigations may directly point to one or a few candidate genes, enabling faster and cheaper diagnosis by targeted gene analysis (5-7). In practice, clinical characterization and WES go hand-in-hand (8, 9). Clinical data are essential not only to guide WES variant selection, but also to define the impact and severity a genetic defect may have.

We investigated a patient in whom MRI and WES studies enforced each other in interpreting the genetic defect and establishing a diagnosis. We found that in this case MRI data are predictive for the severity of the clinical manifestations and can be used to identify severe cases of Perrault syndrome due to CLPP mutations.

\section{Material and Methods}

\section{Study design}

We performed WES on a patient (patient 1.1) from consanguineous parents who suffered from pronounced neurological problems and had specific abnormalities on brain-MRI, comparable to his affected sibling (patient 1.2). Subsequently, the Amsterdam brain-MRI database containing over 3000 unclassified leukoencephalopathy cases was checked manually by experts to identify patients with a similar MRI pattern. This resulted in selection of three unrelated patients (patient 2, patient 3.1 and patient 4) who were then, together with their affected siblings (patient 3.2), subject to targeted CLPP gene analysis.

\section{METc}

Procedures involving human participants were in accordance with the ethical standards of the institutional (MUMC+, VUmc) research committee. All subjects gave written informed consent in accordance with the Declaration of Helsinki. 


\section{Patients}

The clinical details of the patients with CLPP mutations were retrospectively reviewed.

\section{Magnetic Resonance Imaging}

For all patients at least one MRI was available for review. The studies had been performed on different MRI machines of different vendors, precluding quantitative measurements, but allowing MRI pattern analysis. In all patients axial T2-weighted images were used for systematic analysis of the abnormalities (10). Based on the MRI findings in the first patient, the MRI criteria for further inclusion of patients were: signal abnormalities in the deep and subcortical cerebral white matter, signal abnormalities in the middle blade of the corpus callosum and, absence of abnormalities in basal nuclei and thalami.

\section{Homozygosity mapping}

Peripheral blood DNA was prepared and labelled for Affymetrix Human Mapping 250K array according to the manufacturer's protocol (Affymetrix GeneChip, Santa Clara, CA, USA).

\section{WES analysis}

Agilent SureSelect version 4 (Agilent Technologies, Santa Clara, CA, USA) was used for exome enrichment, and massive parallel sequencing was performed on an Illumina HiSeq2000 platform, using a 2x100 bp paired end setting (Illumina, San Diego, CA, USA). Exome data of proband 1.1 were filtered for homozygous variants in homozygosity regions. Only variants with allele frequencies lower than 1\% (dbSNP137), consisting of non-synonymous substitutions, INDELs (in-frame and frameshift), nonsense mutations and splice-variants, were evaluated. Non-annotated variants were maintained, unless allele frequencies exceeded $5 \%$ prevalence in our in-house patient database. Enrichment for missense variants in conserved protein domains was ensured by removal of variants with PhyloP values <3.5. Pathogenicity of non-synonymous missense mutations was estimated by PolyPhen-2 (11), SIFT (12), PROVEAN (13) and Mutation Taster (14).

\section{Sanger sequencing and paternity testing}

CLPP exons and flanking intronic regions were PCR amplified using M13-tailed primers (Supplementary Table S1), and sequenced by the ABI 3730 automatic sequencer (Applied Biosystems, Foster City, CA, USA). CLPP Sanger sequencing was performed in all patients, 
sibs and parents. Paternity was tested by multiplex VNTR analysis using the AmpFISTR Identifiler PCR amplification kit according to manufacturer's manual (Applied Biosystems, Foster City, CA, USA).

\section{CLPP mRNA expression}

RNA was isolated from patient and control skin fibroblasts using the MagMAX-96 Total RNA Isolation Kit (ThermoFisher scientific, Waltham, MA, USA). cDNA was synthesized by qScript cDNA Synthesis Kit (Quanta Biosciences, Gaithersburg, MD, USA) using $1 \mu \mathrm{g}$ RNA input. mRNA expression was measured by the $7900 \mathrm{HT}$ Fast Real-Time PCR System (Applied Biosystems, Foster City, CA, USA) based on a primer pair at the 5' side (exon 2 and 3, 104-bp amplicon) and 3' side (exon 5 and 6, 170-bp amplicon) of the CLPP transcript (NM_006012). CLPP expression was normalized against the TBP housekeeping gene (TATA box-binding protein, NM_003194, primer set in exon 5 and 6, 89-bp amplicon) (Supplementary Table S1). Sensimix Sybr Hi-Rox (Bioline, Taunton, MA, USA) was used for cDNA amplification and a relative CLPP mRNA expression was calculated using the $2^{-\triangle \Delta \mathrm{ct}}$ method, normalizing to heathy control fibroblasts. Statistical significance was calculated using a T-TEST (one-tailed, two-sample equal variance) with Bonferroni correction.

\section{Protein structure modeling}

An X-Ray structure of the human mitochondrial CLPP heptamer (PMID: 15522782) at 2.10A resolution has been used for visualizing mutations in the protein structure. The structure was displayed in PyMol (ver.1.8) and mutations were introduced with the Mutalizer package.

\section{mtDNA and CLPP copy number quantification}

mtDNA copy number was quantified by comparing mtDNA encoded ND5 copy number (mtDNA NADH dehydrogenase 5, NC_012920.1) with the nuclear B2M gene (nuclear gene beta-2-microglobulin, NC_000015.10)(Supplementary Table S1). DNA amplification was performed under similar conditions as reported above. A relative mtDNA copy number was defined using the $2^{-\Delta \Delta c t}$ method, normalizing to heathy control fibroblasts. Statistical significance was calculated using a T-TEST (one-tailed, two-sample equal variance) with Bonferroni correction.

A similar method was used to determine the CLPP nDNA copy number (NC_000019.10). A primer pair located in intron 2 and exon 3 (primer set exon 3, 176-bp amplicon) and in intron 5 and exon 6 (primer set exon 6, 168-bp amplicon) of the CLPP gene were used. 
Two amplicons were designed to cover part of exon 4, a primer-pair in intron 3 and exon 4 (primer set 4A; 135bp amplicon) and exon 4 and intron 4 (primer set 4B; 164bp amplicon) (Supplementary Table S1).

\section{Results}

\section{Patients}

Supplementary Table S2 contains the clinical details of all patients. The proband, patient 1.1 (born in 1993), was the $2^{\text {nd }}$ child from consanguineous parents. He presented with congenital sensorineural deafness, psychomotor retardation, epilepsy and small stature. He had a younger brother, patient 1.2 (born in 1995), with a similar clinical picture. Patient 2 (born in 1994) and sibling-patients 3.1 and 3.2 (born in 1989 and 1994, respectively) also had early onset deafness, stunted growth, developmental retardation of variable severity and motor problems due to ataxia, spasticity and peripheral neuropathy. Two of the patients had epilepsy. In all patients the disease course was slowly progressive and in some of them provoking factors like febrile infections could lead to episodes of regression.

\section{Magnetic Resonance Imaging}

Table 1 contains the MRI details of all patients, also illustrated in Figure 1. All patients had inhomogeneous, partially multifocal and partially confluent signal abnormalities in the deep and subcortical white matter, largely sparing the directly periventricular white matter. In all patients the middle blade of the corpus callosum was affected, while the inner and outer blades were spared. Only one patient (patient 2) showed signal abnormalities in the cerebellar white matter and central tegmental tracts in the pons, while two patients had signal abnormalities in the middle cerebellar peduncles. Over time cerebral and less severe cerebellar atrophy occurred.

Patient 4, who did not have CLPP mutations, had more extensive cerebral white matter abnormalities at disease onset and striking swelling of the corpus callosum. In the course of a few years the white matter signal abnormalities largely disappeared, although the middle blade of the corpus callosum remained abnormal. He developed very mild atrophy. 
Table 1: MRI findings.

\begin{tabular}{|c|c|c|c|c|c|}
\hline Patient number & Patient 1.1 & patient 1.2 & Patient 2 & Patient 3.1 & Patient 3.2 \\
\hline Year of birth & 1995 & 1993 & 1994 & 1989 & 1994 \\
\hline MRIs (ages in years) & $2(9,10,12)$ & $1(8)$ & $4(3,11,12,17,20)$ & $2(11,20)$ & $1(4)$ \\
\hline \multicolumn{6}{|l|}{ First MRI } \\
\hline $\begin{array}{l}\text { Cerebral WM abna, } \\
\text { aspect }\end{array}$ & $\begin{array}{l}\text { inhomogeneous, } \\
\text { partially multifocal }\end{array}$ & multifocal & $\begin{array}{l}\text { inhomogeneous, } \\
\text { partially multifocal }\end{array}$ & $\begin{array}{l}\text { inhomogeneous, } \\
\text { partially multifocal }\end{array}$ & $\begin{array}{l}\text { inhomogeneous, } \\
\text { partially multifocal }\end{array}$ \\
\hline $\begin{array}{l}\text { Predominance cerebral } \\
\text { WM abn. }\end{array}$ & deep + subcortical & deep & deep + subcortical & deep + subcortical & deep + subcortical \\
\hline Sparing cerebral WM & periventricular & $\begin{array}{l}\text { periventricular+ } \\
\text { subcortical }\end{array}$ & periventricular & periventricular & periventricular \\
\hline $\begin{array}{l}\text { Corpus callosum genu } \\
\text { abn. }\end{array}$ & $\begin{array}{l}+ \text {, esp. middle } \\
\text { blade }\end{array}$ & + , middle blade & + , esp. middle blade & $\begin{array}{l}+ \text {, esp. middle } \\
\text { blade }\end{array}$ & $\begin{array}{l}+ \text {, esp. middle } \\
\text { blade }\end{array}$ \\
\hline $\begin{array}{l}\text { Corpus callosum body } \\
\text { abn. }\end{array}$ & - & - & - & - & - \\
\hline $\begin{array}{l}\text { Corpus callosum } \\
\text { splenium abn. }\end{array}$ & + & + , middle blade & + & - & + \\
\hline $\begin{array}{l}\text { Internal/external } \\
\text { capsule abn. }\end{array}$ & $-1-$ & $-1-$ & $-1-$ & $-1-$ & $-1-$ \\
\hline Swelling of abn. WM & - & - & - & - & - \\
\hline $\begin{array}{l}\text { Rarefaction of abn. } \\
W^{b}{ }^{b}\end{array}$ & - & - & + & - & - \\
\hline WM cysts ${ }^{c}$ & - & - & - & - & - \\
\hline $\begin{array}{l}\text { Cerebral cortex signal } \\
\text { abn. }\end{array}$ & - & - & - & - & - \\
\hline Basal nuclei signal abn. & - & - & - & - & - \\
\hline Thalamus signal abn. & - & - & - & - & - \\
\hline Cerebral atrophy & slight & - & - & slight & - \\
\hline $\begin{array}{l}\text { Cerebellar WM signal } \\
\text { abn. }\end{array}$ & - & - & + & - & - \\
\hline $\begin{array}{l}\text { Hilus dentate nucleus } \\
\text { abn. }\end{array}$ & - & - & - & - & - \\
\hline $\begin{array}{l}\text { Cerebellar cortex signal } \\
\text { abn. }\end{array}$ & - & - & - & - & - \\
\hline $\begin{array}{l}\text { Dentate nucleus signal } \\
\text { abn. }\end{array}$ & - & - & - & - & - \\
\hline Cerebellar atrophy & - & - & - & - & - \\
\hline $\begin{array}{l}\text { Middle cerebellar } \\
\text { peduncles abn. }\end{array}$ & - & + & + & - & - \\
\hline Brainstem signal abn. & - & - & $\begin{array}{l}+ \text {, central tegmental } \\
\text { tracts }\end{array}$ & - & - \\
\hline Brain stem atrophy & - & - & - & - & - \\
\hline Contrast enhancement & - & n.d. & n.d. & n.d. & n.d. \\
\hline Restricted diffusion $^{d}$ & n.d. & n.d. & n.d. & n.d. & n.d. \\
\hline Elevated lactate in MRS & - & n.d. & n.d. & n.d. & n.d. \\
\hline \multicolumn{6}{|l|}{ Latest MRI } \\
\hline Change over time & $\begin{array}{l}\text { slight further } \\
\text { cerebral atrophy }\end{array}$ & & $\begin{array}{l}\text { cerebral and } \\
\text { cerebellar atrophy; no } \\
\text { diffusion restriction; } \\
\text { no contrast } \\
\text { enhancement }\end{array}$ & $\begin{array}{l}\text { cerebral and } \\
\text { cerebellar atrophy, } \\
\text { cerebellar WM } \\
\text { abn., no diffusion } \\
\text { restriction }\end{array}$ & - \\
\hline
\end{tabular}

a Signal abnormalities defined as an abnormally high signal on $\mathrm{T}_{2}$-weighted images

${ }^{b}$ White matter rarefaction defined as $\mathrm{T}_{2 .}$ hyperintense WM with low signal on FLAIR, but not as low as cerebrospinal fluid

'Cysts defined as $\mathrm{T}_{2}$-hyperintense WM with low signal on FLAIR, as low as cerebrospinal fluid

${ }^{d}$ Restricted diffusion assessed using the apparent diffusion coefficient (ADC) to avoid $\mathrm{T}_{2}$-shine-through effects

$\mathrm{WM}=$ white matter; $\mathrm{abn}$. = abnormalities / abnormal; $\mathrm{MRS}=$ magnetic resonance spectroscopy; nd = not done; esp. = especially; + = present; - = absent; FLAIR = fluid attenuated inversion recovery 


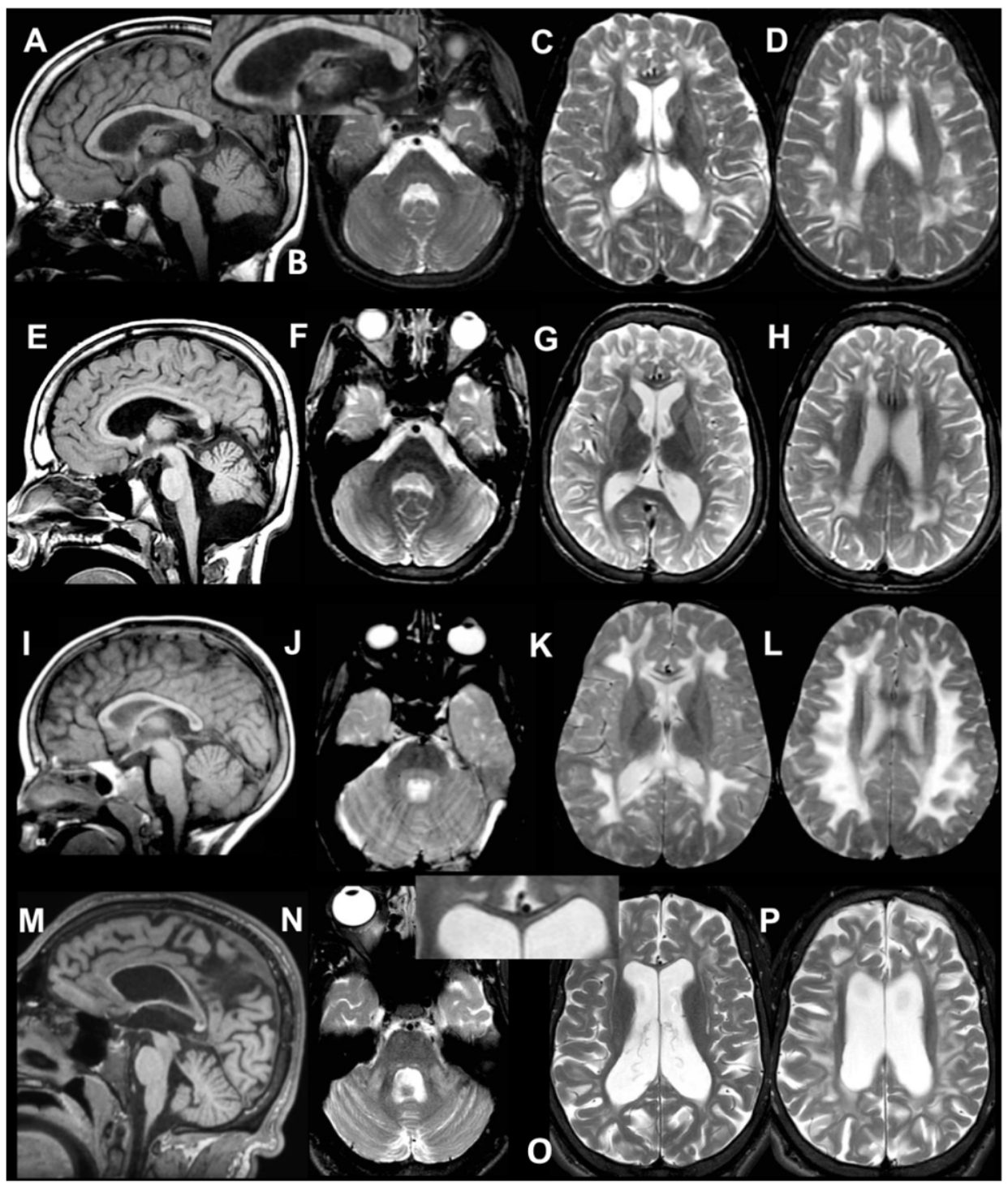

Figure 1: MRI of patient 1.1 at 9 years $(A-D)$ and 12 years $(E-H)$. The first $M R I$ shows signal abnormalities in the middle blade of the corpus callosum (A, $C$ and inset in A); inhomogeneous mainly multifocal signal abnormalities in the subcortical and deep cerebral white matter, sparing a periventricular rim $(C, D)$; and slight cerebral $(C, D)$ but no cerebellar atrophy $(A, B)$. At follow-up, slightly increased cerebral and cerebellar atrophy is seen. MRI of patient 2 at 4 years (I-L) and 20 years (M-P). The first MRI shows signal abnormalities in the middle blade of the corpus callosum (I,K), inhomogeneous, mainly confluent signal abnormalities in the subcortical and deep cerebral white matter, sparing a periventricular rim $(K, L)$ and no cerebral or cerebellar atrophy (I-L). At follow-up, severe cerebral and milder cerebellar atrophy is seen (M-P). The corpus callosum is highly atrophic, but the selective involvement of the middle blade is still seen (inset in $\mathrm{O}$ ). Within the posterior fossa, signal abnormalities are present in the middle cerebellar peduncles and pontine central tegmental tracts $(N)$. 


\section{WES and CLPP analysis}

WES of patient 1.1 (family 1) resulted, after filtering, in 34 homozygous variants. His brother, patient 1.2 , was affected as well and homozygosity mapping showed a $56 \mathrm{Mb}$ overlap between the siblings, resulting in 19 candidate genes. Candidate mutations were further limited to evolutionary conserved protein positions. The most promising mutation was a novel (non-annotated) homozygous mutation in a highly conserved domain of the CLPP gene (NM_006012_c.21delA, chr: 19, g.6361606delA). The single nucleotide deletion is located in exon 1 , and is expected to cause a frameshift with a nonsense mutation in exon 3 (p.(Ala10Profs*117)). The resulting "stop" mutation would cause a loss of 160 amino acids of the evolutionary highly conserved CLPP protein, and is likely to result in nonsense mediated mRNA decay. Indeed, CLPP mRNA expression was decreased to approximately $50 \%$ in patient fibroblasts compared to control fibroblasts (Figure $2 \mathrm{~A}$ ), confirming a role for nonsense mediated decay. As it has been reported in mice studies that CLPP mutations affect mtDNA copy number, we determined this in fibroblast DNA from patient 1.1(15). Patient 1.1 showed an approximately three-fold increase in mtDNA copy-number (Figure $2 B)$. Mutation segregation testing in family 1 indicated that the unaffected parents and sister were heterozygous and the affected brother was homozygous for the variant.

A

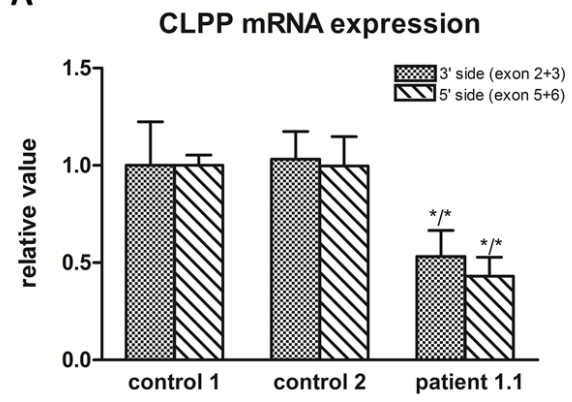

B

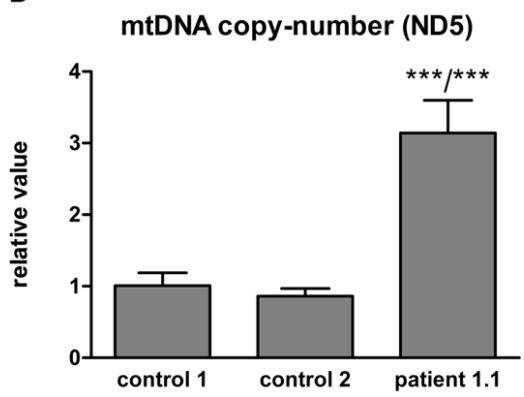

Figure 2: (A) CLPP MRNA expression. CLPP expression was measured using a primer pair at the $3^{\prime}$ side (exon 2 and 3) and 5' side (exon 5 and 6) of the transcript (NM_006012), and was normalized to TBP. Approximately 50\% lower CLPP mRNA levels were found in patient 1.1 fibroblasts compared to controls. (B) mtDNA copy-number. mtDNA copy-number was measured based on ND5 copy-number and was normalized to nuclear B2M. Approximately a 3-fold copy-number increase was measured in patient 1.1 (proband) fibroblasts compared to control fibroblasts.

Patient 2 (family 2) carried an apparently homozygous c.484G>A (NM_006012.2_c.484G>A; chr: 19; g.6364579G>A) missense mutation in exon 4, causing an amino acid substitution at p.(Gly162Ser), which is located at the border of a helix domain (p.154-160; Nextprot: EC 3.4.21.92). The mutation affects an evolutionary conserved protein domain (PhyloP: 5.61) 
and was predicted harmful at the protein level (SIFT: 0.000, PROVEAN: -5.8, MutationTaster: disease causing). To examine the impact of the p.(Gly162Ser) amino acid substitution on CLPP protein structure, template based in silico protein modeling was performed based on the crystal structure of the CLPP heptamer (UNIPROT accession number: Q16740, Protein Data Bank identifier 1tg6). The p.(Gly162Ser) mutation in our patient introduces a bulky serine side chain that destabilizes the interaction between two structural domains, the alpha-helix (residues 154-160) and the beta sheet (residues 143-152) (Figure 3A). More importantly, the serine side chain protrudes towards and clashes with the Thr145 amino acid of the beta sheet, a residue in which the p.(Thr145Pro) mutation, resulting in significant disruption of the protein structure in the region, has been reported as disease causative (16). Segregation analysis in the family indicated that patient 2 was hemizygous for the mutation, as the father was heterozygous carrier and the mother did not carry the mutation (wild-type). To test for the presence of a maternal deletion, we determined the CLPP copy-number for regions in exon 3, 4 and 6 in the patient and his parents. The patient's copy number was approximately $50 \%$ lower for exon 3 and exon 4 compared to the father (Figure $4 \mathrm{~A}$ ). The same decrease in copy number was seen in DNA from the mother and healthy sibling, indicating that the deletion was inherited from the mother. Interestingly, the copy number of exon 6 did not significantly differ, which is in line with the presence of a heterozygous SNP in exon 6 of the patient. The maternally inherited deletion is therefore expected not to exceed exon 6.

In patient 3.1 and his affected brother patient 3.2 (family 3), an apparently homozygous c.425C > T (NM_006012.2_ c.425C >T; chr: 19; g.6364520C $>T$ ) missense mutation was identified in exon 4, in an evolutionary highly conserved protein domain (PhyloP: 5.69). It was predicted to have highly pathogenic impact on protein function (SIFT: 0.003, PROVEAN: -8.9, MutationTaster: disease causing). The resulting p.(Pro142Leu) amino acid substitution is located at the border of a Beta-strand (p.143-152; Nextprot: EC 3.4.21.92), near two mutations (p.(Thr145Pro) and p.(Cys147Ser)) that have been included in the HGMD database for disease causing mutations. The p.(Pro142Leu) mutation changes a nonreactive proline residue, located in a loop (139-142) at the boundary with the beta sheet (143-152), into a highly hydrophobic leucine residue (Figure 3B). This loop is localized at the CLPP-CLPX interface which is mediated by the beta sheet structure shown to be affected by the previously reported Thr145Pro mutation and p.(Gly162Ser) mutation, reported here for patient 2. Likely, the drastic hydrophobicity change, caused by the p.(Pro142Leu) mutation, will affect the efficiency of CLPP-CLPX docking due to changes at the CLPP-CLPX interface, a region in which mutations have previously been related to pathogenicity(16). Segregation testing showed that his affected sibling carried the same mutation and that both patients were hemizygous, since only the mother was a heterozygous carrier, whereas the father did not carry the mutation. Non-paternity was excluded. CLPP copy-numbers for regions in exon 
3, 4 and 6 were determined for patient 3.1 and his parents (Figure 4B). An approximately $50 \%$ decrease in copy-number of exon 3, exon 4 and exon 6 was observed in the patient and his father, but not his mother. The genomic deletion, which is transmitted by the father, is therefore expected to cover at least exon 3 to exon 6 of the CLPP gene.

In patient 4 no mutations were identified in the CLPP gene.

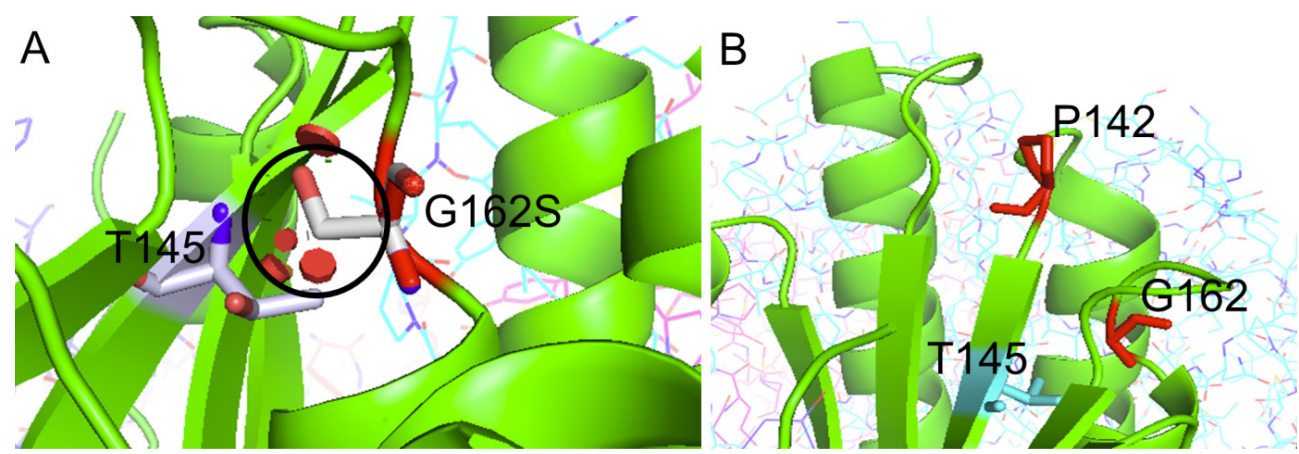

Figure 3: Crystal-structure based in silico modeling of the CLPP protein (Uniprot accession number: Q16740). (A) p.(Gly162Ser) introduces a long serine side chain that interacts with the Thr145 amino acid and destabilizes the interaction between the alpha-helix structure (residues 154-160) and beta sheet (residues 143-152). Red discs indicate significant van der Waals overlap, including a conflict with a p.145 position previously reported in CLPP patients. (B) p.(Pro142Leu) changes a proline residue, located in a loop (139-142) at the boundary with a beta sheet (143-152), into a highly hydrophobic Leucine residue. This drastic hydrophobicity change could interfere with CLPP-CLPX binding at the interface.
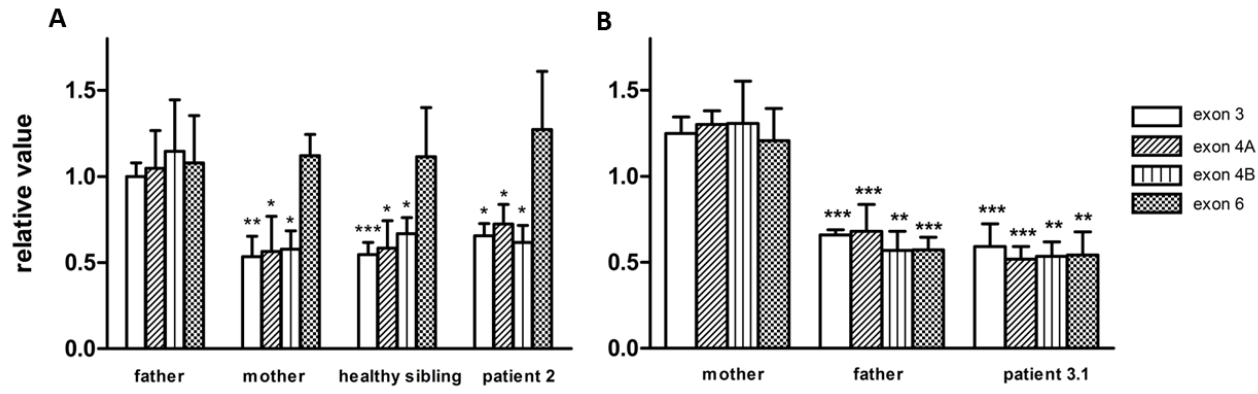

Figure 4: The CLPP nDNA copy-number. CLPP copy-number was determined based on amplicons in exon3, exon 4 and exon 6 and was normalized to B2M. Copy numbers were relative to control DNA. (A) In family 2, CLPP copy-numbers of patient 2, the healthy sibling and mother were compared to the heterozygous father and indicated a maternally inherited CLPP deletion. (B) In family 3, CLPP copy numbers of patient 3.1 and the father were compared to the heterozygous mother and indicated a paternally inherited CLPP deletion. 


\section{Discussion}

Using WES, we identified a novel homozygous single nucleotide deletion (c.21delA) in the CLPP gene of two affected siblings of family 1 . The resulting frameshift was expected to introduce a premature stop codon in exon 3. Quantification of CLPP transcript expression in patient fibroblasts showed an approximately 50\% decrease in CLPP mRNA levels, indicating nonsense mediated decay. CLPP plays a role in the mitochondrial unfolded Protein Response pathway (mtUPR) by multimerizing with the CLPX chaperone within the mitochondrial matrix, thereby forming a proteasome-like cylinder that is capable of hydrolyzing mitochondrial proteins into small peptides. It is therefore expected to react upon protein conformational stress in mitochondria by inducing mitochondrial stress signaling pathways $(17,18)$. Molecular characterization of Clpp null mice has demonstrated that absence of Clpp transcripts leads to significant increases in mtDNA copy number in several tissues (testis, ovary, heart and brain) (15). We confirmed an approximately three-fold increase in mtDNA copy-number in fibroblasts of patient 1.1, indicating that the c.21delA mutation was likely to affect CLPP function in the same way.

We used the brain-MRI of patient 1.1 as a template to screen an Amsterdam brainMRI database containing over 3000 unclassified leukoencephalopathy for comparable characteristics, and selected three patients based on similarities in brain MRI and clinical manifestations for targeted CLPP sequencing (19). In two of these patients we identified harmful missense mutations in CLPP. In the third patient no CLPP mutation was identified. This patient initially fulfilled the MRI criteria, but over the years the white matter abnormalities improved and largely disappeared without significant cerebral or cerebellar atrophy. A p.(Gly162Ser) mutation in patient 2 and p.(Pro142Leu) mutation in patient 3.1 and 3.2 were located at an evolutionary highly conserved amino-acid position and were predicted to be pathogenic according to several in silico prediction tools. Crystal-structure based protein modeling showed that the p.(Gly162Ser) mutation is likely to destabilize the interaction between an alpha-helix structure and beta sheet and could therefore disrupt CLPP protein structure. The mutation interferes with the amino acid p.Thr145, a residue in which a disease causing mutation was previously reported in a patient with pronounced neurological symptoms (Table 2). Also changes in hydrophobicity of the p.(Pro142Leu) substitution, located adjacent to this beta sheet, could interfere with the CLPP-CLPX binding-interface (20). Changes at the CLPP-CLPX interface have previously been suggested to be involved in disease (16). CLPP copy number quantification in fibroblasts of patient 2 and patient 3.1 showed that both mutations were actually hemizygous. Whereas the CLPP deletion in patient 2 was maternally inherited and did not exceed exon 6; the deletion in patient 3.1 was paternally inherited and covered at least exon 1 to 6 . Our data indicate that 
large deletions can be responsible for a substantial part of the inherited CLPP defects and that one should be aware that these mutations could be missed in purely sequencing-based approaches.

Our patients, who were all male, suffered from heterogeneous manifestations, involving congenital sensorineural hearing loss ( $\mathrm{SNHL}$ ), psychomotor retardation, ataxia, autism, epilepsy and short stature. Comparable symptoms have previously been reported in seven other families with CLPP defects (Table 2) (16, 21-24). Apart from the consistent involvement of sensorineural hearing loss, sex-related clinical features have been reported such as female ovarian dysgenesis and infertility due to azoospermia in males $(15,22)$. Our male patients were not tested for infertility. Consequently, CLPP mutations cause highly heterogeneous symptoms that have been classified as Perrault syndrome type 3 (PRLTS3). T2-weighted brain-MRI of our patients showed inhomogeneous signal abnormalities in the deep and subcortical cerebral white matter and middle blade of the corpus callosum, with over time severe cerebral atrophy. However, neurological implications are not always pronounced in PRLTS3. Whereas two earlier reported families showed severe neurological symptoms and white matter abnormalities on MRI, other families showed a much milder phenotype. Previously, Jenkinson et al. explained differences in clinical impact by the more drastic structural protein changes caused by the p.(Thr145Pro) mutation in comparison to p.(Cys147Ser). The c. $270+4 A>G$ splice site mutation was shown to cause only partial ablation of donor splice site function and was therefore likely to have residual CLPP activity (Table 2) (16). Our data support the observation that severe neurological PRLTS3 phenotypes are caused by mutations that drastically affect the native CLPP protein structure and show that large deletions might be responsible for a substantial part of these CLPP defects. 
Table 2: Indicates the neurological implications of CLPP mutations in our patients (family 1-3) and earlier reported CLPP cases (family 4-10).

\begin{tabular}{|c|c|c|c|}
\hline Family & $\begin{array}{l}\text { Pronounced } \\
\text { Neurological } \\
\text { symptoms }\end{array}$ & $\begin{array}{l}\text { White matter } \\
\text { abnormalities } \\
\text { (MRI) }\end{array}$ & Mutations \\
\hline $\begin{array}{l}1 \text { consanguineous } \\
\text { Patient } 1.1 \text { and } 1.2\end{array}$ & yes & yes & $\begin{array}{l}\text { - } \quad \text { NM_006012_c.21delA (homozygous) } \\
\text { - } \quad \text { frameshift with non-sense mutation } \\
\text { NMD: } 60 \% \text { transcript loss }\end{array}$ \\
\hline $\begin{array}{l}2 \text { non-consanguineous } \\
\text { Patient } 2\end{array}$ & yes & yes & $\begin{array}{ll}- & \text { NM_006012.2_c.484G }>A \\
-\quad & \text { p.(Gly162Ser) (hemizygous) } \\
\text { - } & \text { large allelic CLPP deletion }\end{array}$ \\
\hline $\begin{array}{l}3 \text { non-consanguineous } \\
\text { Patient } 3 \text { and } 3.1\end{array}$ & yes & yes & $\begin{array}{ll}- & \text { NM_006012.2_c.425C }>T \\
-\quad & \text { p.(Pro142Leu) (hemizygous) } \\
\text { - } & \text { large allelic CLPP deletion }\end{array}$ \\
\hline \multicolumn{4}{|l|}{ Literature reported } \\
\hline $\begin{array}{l}4 \text { consanguineous } \\
3 \text { patients }\end{array}$ & $\begin{array}{l}\text { yes; the } 8 \text { month } \\
\text { old boy did not } \\
\text { show signs of } \\
\text { PRLTS3 yet }\end{array}$ & yes (eldest sibs) & $\begin{array}{ll}- & \text { c.685T>G } \\
\text { - } & \text { p.(Tyr229Asp) (homozygous) }\end{array}$ \\
\hline $\begin{array}{l}5 \text { consanguineous } \\
3 \text { patients }\end{array}$ & yes & $\begin{array}{l}\text { yes (single } \\
\text { patient analyzed) }\end{array}$ & $\begin{array}{ll}\text { - } & \text { c. } 433 \mathrm{~A}>\mathrm{C} \\
\text { - } & \text { p.(Thr145Pro) (homozygous) }\end{array}$ \\
\hline $\begin{array}{l}6 \text { consanguineous } \\
4 \text { patients }\end{array}$ & no & n.d. & $\begin{array}{ll}\text { - } & \text { c. } 440 \mathrm{G}>\mathrm{C} \\
& \text { p.(Cys147Ser) (homozygous) }\end{array}$ \\
\hline $\begin{array}{l}7 \text { consanguineous } \\
2 \text { patients }\end{array}$ & no & n.d & $\begin{array}{ll}- & \text { c.439T>A } \\
\text { - } & \text { p.(Cys147Ser) (homozygous) }\end{array}$ \\
\hline $\begin{array}{l}8 \text { consanguineous } \\
3 \text { patients }\end{array}$ & no & n.d. & $\begin{array}{ll}\text { - } & \text { c. } 270 p(+) 4 A>G \text { (homozygous) } \\
\text { - } & \text { splice donor site mutation } \\
& \text { weakens donor-splice site function }\end{array}$ \\
\hline $\begin{array}{l}9 \text { consanguineous } \\
1 \text { patient }\end{array}$ & no & no & $\begin{array}{ll}\cdot & \text { c.430T>C } \\
\cdot & \text { p.(Cys144Arg) (homozygous) }\end{array}$ \\
\hline $\begin{array}{l}10 \text { unknown (ahead of } \\
\text { print) } \\
2 \text { patients }\end{array}$ & no & n.d. & $\begin{array}{ll}\text { - } & \text { c. } 624 \mathrm{C}>\mathrm{G} \\
& \text { p.(lle208Met) (homozygous) }\end{array}$ \\
\hline
\end{tabular}

Whereas symptoms such as SNHL might manifest during early childhood with gradual progression and variable impact, indications for infertility such as impaired sex hormone profiles can only be detected after puberty. Age-dependent symptom presentation and phenotypic heterogeneity can therefore impede genetic diagnosis, especially during early disease onset when only part of the disease manifestations is present. We demonstrate that similarity in brain-MRI pattern of abnormalities among patients can be successfully used to identify other PRLTS3 patients, already at early disease onset. Our selection criteria were signal abnormalities predominantly involving both the subcortical and deep cerebral white matter and the middle blade of the corpus callosum. Multifocal and confluent abnormalities predominantly involving the subcortical and deep cerebral white matter, 
sparing a periventricular rim, are also seen in L-2-hydroxyglutaric aciduria, Kearns Sayre syndrome and 'Leukoencephalopathy with thalamus and brain stem abnormalities and lactate elevation' and Canavan disease $(19,25)$. However, involvement of the subcortical and deep cerebral white matter is typically associated with sparing of the corpus callosum or involvement of its outer blade. The combination of abnormalities predominantly involving the subcortical and deep cerebral white matter and the middle blade of the corpus callosum is so specific for PRLTS3 that it allowed us to select patients by MRI criteria only. However, since not all PRLTS3 patients suffer from neurological symptoms, this approach may only apply to severely affected cases.

\section{Acknowledgement}

This work was supported by Princess Beatrix Spierfonds grant application no. W.OR11-24, ZonMw TOP grant 91211005, Stichting MetaKids and the Province of Limburg. 


\section{References}

1. Khalifa M, Naffaa L. Exome sequencing reveals a novel WDR45 frameshift mutation and inherited POLR3A heterozygous variants in a female with a complex phenotype and mixed brain MRI findings. Eur J Med Genet. 2015;58(8):381-6.

2. Cullinane AR, Vilboux T, O'Brien K, Curry JA, Maynard DM, Carlson-Donohoe H, et al. Homozygosity mapping and whole-exome sequencing to detect SLC45A2 and G6PC3 mutations in a single patient with oculocutaneous albinism and neutropenia. J Invest Dermatol. 2011;131(10):201725.

3. Netravathi M, Kumari R, Kapoor S, Dakle P, Dwivedi MK, Roy SD, et al. Whole exome sequencing in an Indian family links Coats plus syndrome and dextrocardia with a homozygous novel CTC1 and a rare HES7 variation. BMC Med Genet. 2015;16:5.

4. Craigen WJ, Graham BH, Wong LJ, Scaglia F, Lewis RA, Bonnen PE. Exome sequencing of a patient with suspected mitochondrial disease reveals a likely multigenic etiology. BMC Med Genet. 2013;14:83.

5. Huemer M, Karall D, Schossig A, Abdenur JE, Al Jasmi F, Biagosch C, et al. Clinical, morphological, biochemical, imaging and outcome parameters in 21 individuals with mitochondrial maintenance defect related to FBXL4 mutations. Journal of inherited metabolic disease. 2015;38(5):905-14.

6. D'Gama AM, Pochareddy S, Li M, Jamuar SS, Reiff RE, Lam AT, et al. Targeted DNA Sequencing from Autism Spectrum Disorder Brains Implicates Multiple Genetic Mechanisms. Neuron. 2015;88(5):910-7.

7. Carrozzo R, Verrigni D, Rasmussen $M$, de Coo R, Amartino H, Bianchi M, et al. Succinate-CoA ligase deficiency due to mutations in SUCLA2 and SUCLG1: phenotype and genotype correlations in 71 patients. J Inherit Metab Dis. 2016;39(2):243-52.

8. Allen NM, Conroy J, Shahwan A, Lynch B, Correa RG, Pena SD, et al. Unexplained early onset epileptic encephalopathy: Exome screening and phenotype expansion. Epilepsia. 2016;57(1):e12-7.

9. Daud D, Griffin H, Douroudis K, Kleinle S, Eglon G, Pyle A, et al. Whole exome sequencing and the clinician: we need clinical skills and functional validation in variant filtering. J Neurol. 2015;262(7):1673-7.

10. van der Knaap MS, Breiter SN, Naidu S, Hart AA, Valk J. Defining and categorizing leukoencephalopathies of unknown origin: MR imaging approach. Radiology. 1999;213(1):12133.

11. Adzhubei I, Jordan DM, Sunyaev SR. Predicting functional effect of human missense mutations using PolyPhen-2. Curr Protoc Hum Genet. 2013; Chapter 7:Unit7 20.

12. Kumar P, Henikoff S, Ng PC. Predicting the effects of coding non-synonymous variants on protein function using the SIFT algorithm. Nat Protoc. 2009;4(7):1073-81.

13. Choi Y, Chan AP. PROVEAN web server: a tool to predict the functional effect of amino acid substitutions and indels. Bioinformatics. 2015;31(16):2745-7.

14. Schwarz JM, Rodelsperger C, Schuelke M, Seelow D. MutationTaster evaluates disease-causing potential of sequence alterations. Nat Methods. 2010;7(8):575-6.

15. Gispert S, Parganlija D, Klinkenberg M, Drose S, Wittig I, Mittelbronn M, et al. Loss of mitochondrial peptidase Clpp leads to infertility, hearing loss plus growth retardation via accumulation of CLPX, mtDNA and inflammatory factors. Hum Mol Genet. 2013;22(24):4871-87.

16. Jenkinson EM, Rehman AU, Walsh T, Clayton-Smith J, Lee K, Morell RJ, et al. Perrault syndrome is caused by recessive mutations in CLPP, encoding a mitochondrial ATP-dependent chambered protease. Am J Hum Genet. 2013;92(4):605-13. 
17. Broadley SA, Hartl FU. Mitochondrial stress signaling: a pathway unfolds. Trends Cell Biol. 2008;18(1):1-4.

18. Osiewacz HD, Bernhardt D. Mitochondrial quality control: impact on aging and life span - a minireview. Gerontology. 2013;59(5):413-20.

19. Steenweg ME, Ghezzi D, Haack T, Abbink TE, Martinelli D, van BerkelCG, et al. Leukoencephalopathy with thalamus and brainstem involvement and high lactate 'LTBL' caused by EARS2 mutations. Brain : a journal of neurology. 2012;135(Pt 5):1387-94.

20. Kim YI, Levchenko I, Fraczkowska K, Woodruff RV, Sauer RT, Baker TA. Molecular determinants of complex formation between Clp/Hsp100 ATPases and the ClpP peptidase. Nat Struct Biol. 2001;8(3):230-3.

21. Ahmed S, Jelani M, Alrayes N, Mohamoud HS, Almramhi MM, Anshasi W, et al. Exome analysis identified a novel missense mutation in the CLPP gene in a consanguineous Saudi family expanding the clinical spectrum of Perrault Syndrome type-3. J Neurol Sci. 2015;353(1-2):14954.

22. Demain LA, Urquhart JE, O'Sullivan J, Williams SG, Bhaskar SS, Jenkinson EM, et al. Expanding the Genotypic Spectrum of Perrault syndrome. Clin Genet. 2016.

23. Dursun F, Mohamoud HS, Karim N, Naeem M, Jelani M, Kirmizibekmez H. Novel missense mutation in the CLPP gene causes Perrault Syndrome type-3 in a Turkish family. J Clin Res Pediatr Endocrinol. 2016.

24. Lerat J, Jonard L, Loundon N, Christin-Maitre S, Lacombe D, Goizet C, et al. An Application of NGS for Molecular Investigations in Perrault Syndrome: Study of 14 Families and Review of the Literature. Hum Mutat. 2016.

25. van der Knaap MS, Valk J. Magnetic resonance of myelination and myelin disorders. 3rd ed. Heidelberg: Springer; 2005. 


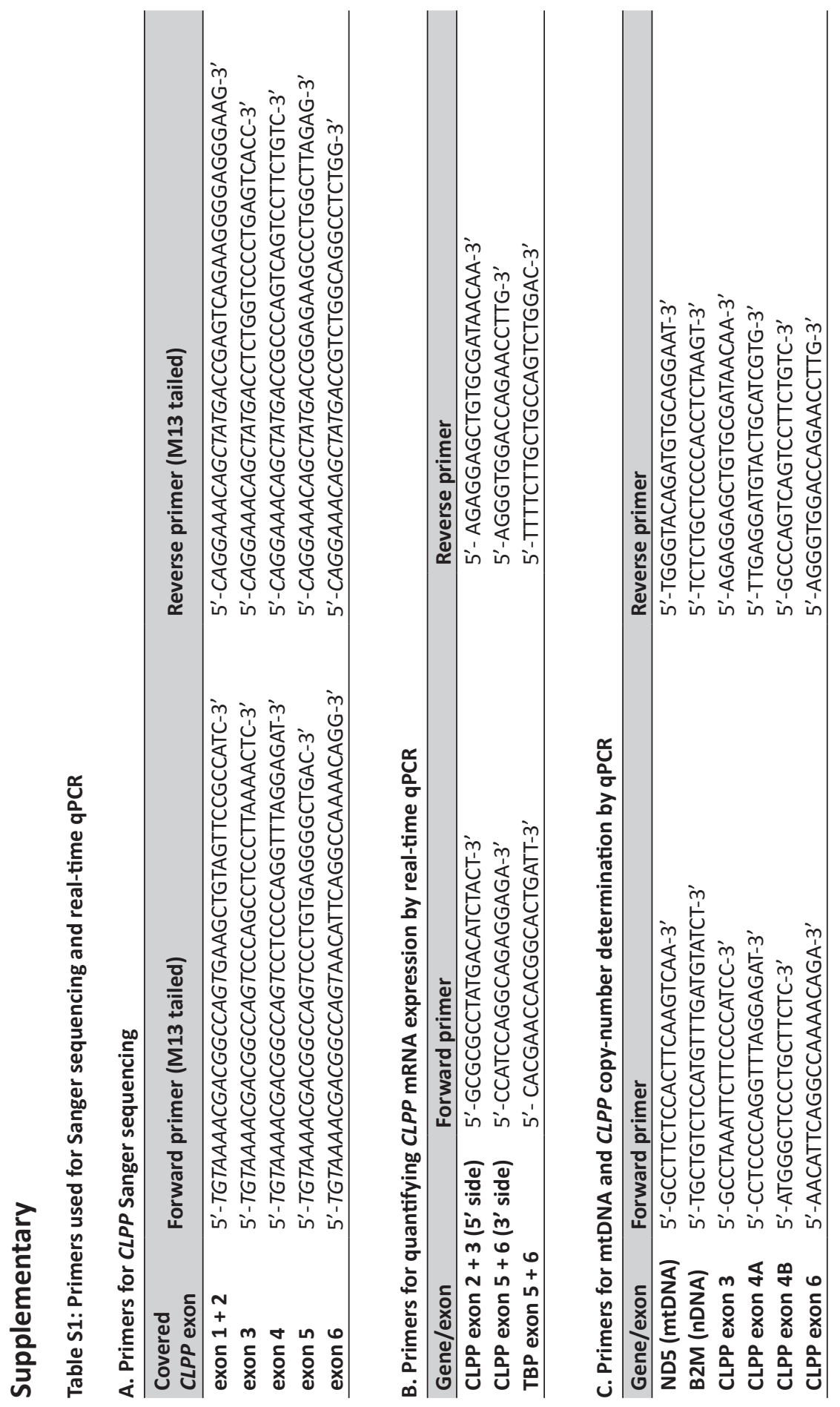




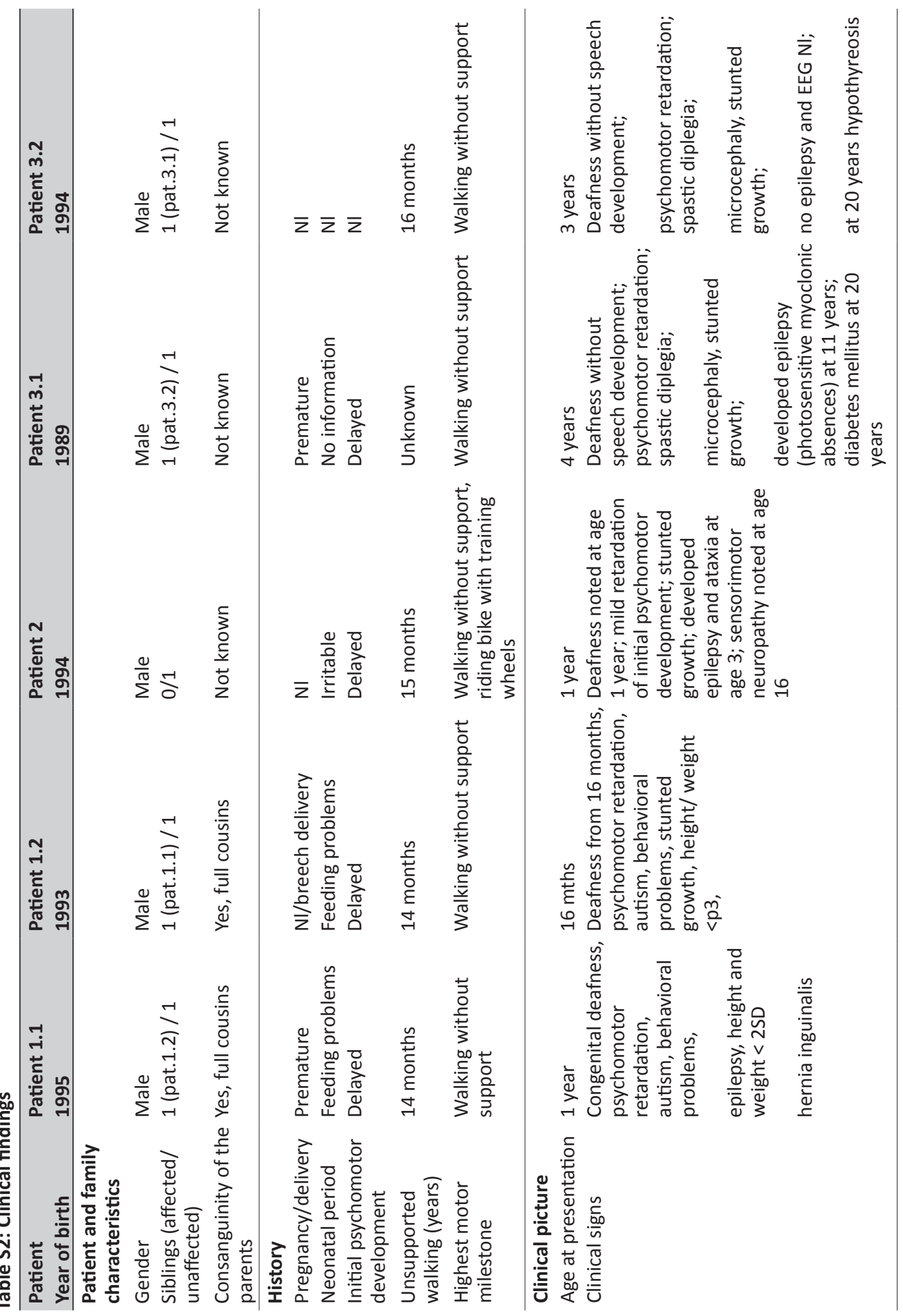




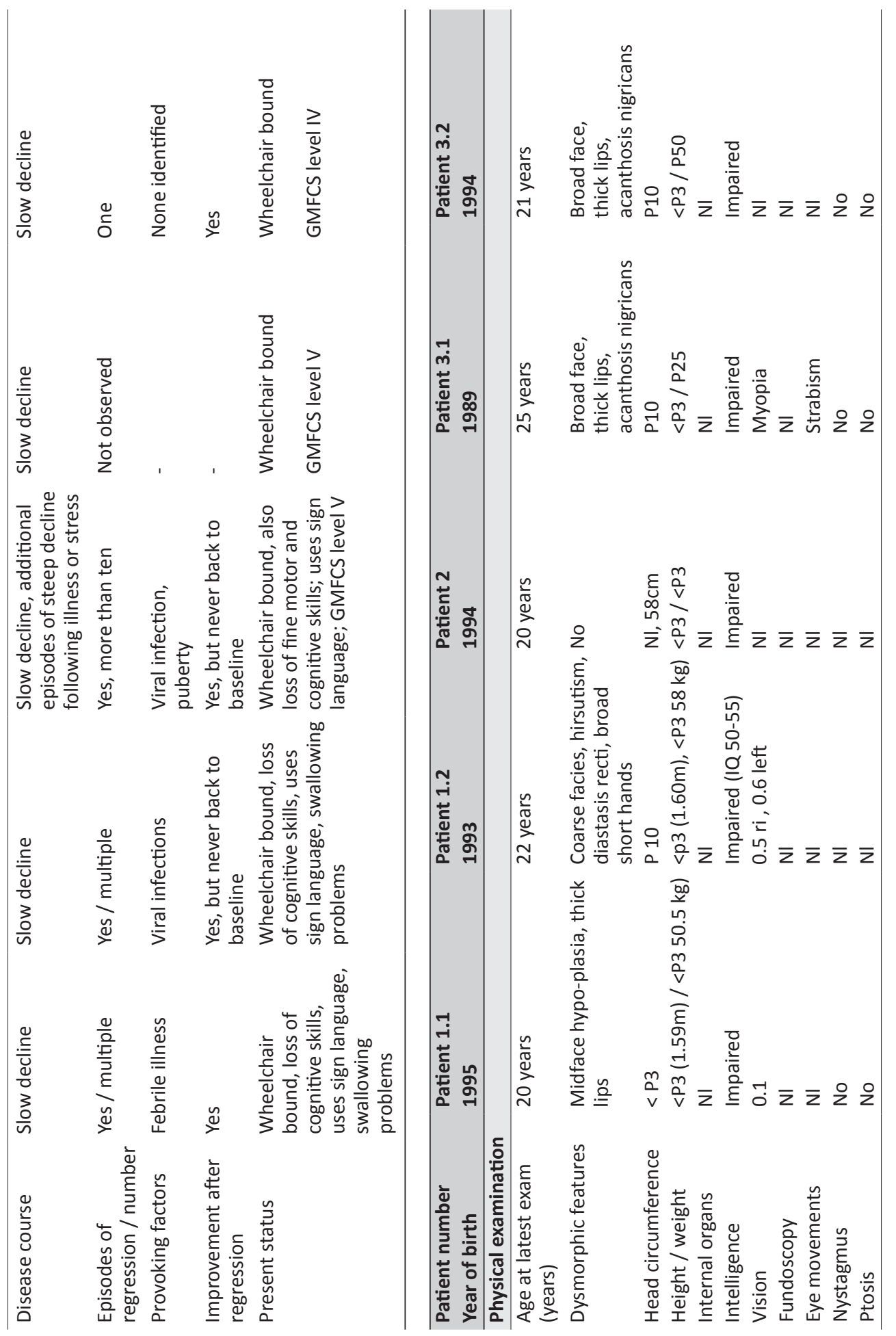


MRI abnormalities reveal PRLTS3 due to CLPP defects

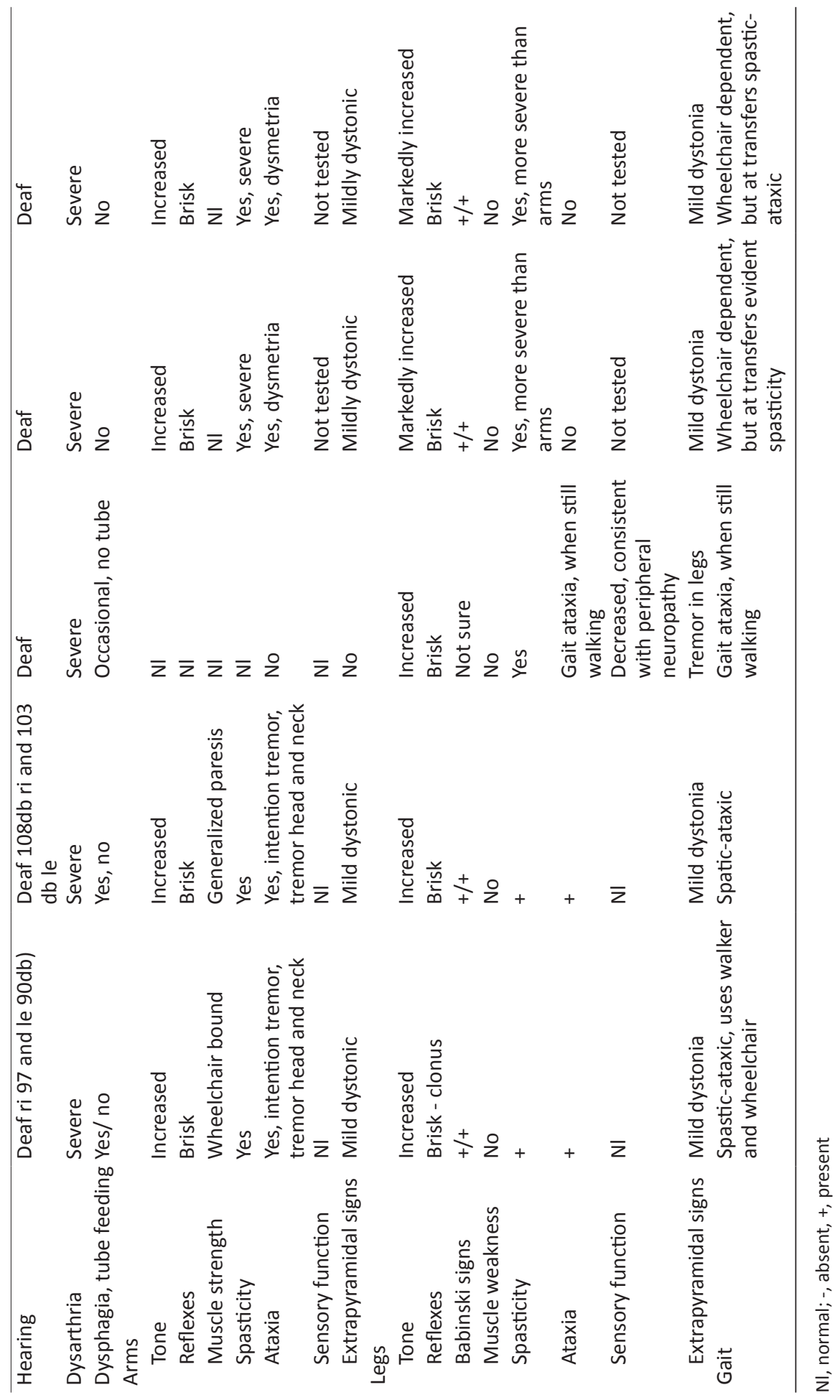





\section{CHAPTER 5}

Selection and characterization of palmitic acid responsive patients with an OXPHOS complex I defect

Tom E.J. Theunissen, Mike Gerards, Debby M. E. I. Hellebrekers, Florence H. van Tienen, Rick Kamps, Suzanne C. E. H. Sallevelt, Elvira N. M. Mulder-Den Hartog, Hans R. Scholte, Robert M. Verdijk, Kees Schoonderwoerd, Irenaeus F. M. de Coo*, Radek Szklarczyk*, Hubert J. M. Smeets*

*= Joint senior authors

Frontiers in molecular neuroscience. 2017;10:336. doi: 10.3389/fnmol.2017.00336 


\begin{abstract}
Mitochondrial disorders are genetically and clinically heterogeneous, mainly affecting high energy-demanding organs due to impaired oxidative phosphorylation (OXPHOS). Currently, effective treatments for OXPHOS defects, with complex I deficiency being the most prevalent, are not available. Yet, clinical practice has shown that some complex I deficient patients benefit from a high-fat or ketogenic diet, but it is unclear how these therapeutic diets influence mitochondrial function and more importantly, which complex I patients could benefit from such treatment. Dietary studies in a complex I deficient patient with exercise intolerance showed increased muscle endurance on a high-fat diet compared to a high-carbohydrate diet. We performed whole-exome sequencing to characterize the genetic defect. A pathogenic homozygous p.G212V missense mutation was identified in the TMEM126B gene, encoding an early assembly factor of complex I. A complementation study in fibroblasts confirmed that the p.G212V mutation caused the complex I deficiency. The mechanism turned out to be an incomplete assembly of the peripheral arm of complex I, leading to a decrease in the amount of mature complex I. The patient clinically improved on a high-fat diet, which was supported by the $25 \%$ increase in maximal OXPHOS capacity in TMEM126B defective fibroblast by the saturated fatty acid palmitic acid, whereas oleic acid did not have any effect in those fibroblasts. Fibroblasts of other patients with a characterized complex I gene defect were tested in the same way. Patient fibroblasts with complex I defects in NDUFS7 and NDUFAF5 responded to palmitic acid, whereas ACAD9, NDUFA12 and NDUFV2 defects were non-responding. Although the data are too limited to draw a definite conclusion on the mechanism, there is a tendency that protein defects involved in early assembly complexes, improve with palmitic acid, whereas proteins defects involved in late assembly, do not. Our data show at a clinical and biochemical level that a high fat diet can be beneficial for complex I patients and that our cell line assay will be an easy tool for the selection of patients, who might potentially benefit from this therapeutic diet.
\end{abstract}




\section{Introduction}

Mitochondrial disorders are genetically and clinically heterogeneous metabolic disorders with a prevalence of $\sim 1$ in 5,000 in the general population (1, 2). Especially high energydemanding organs are affected as a result of impaired oxidative phosphorylation (OXPHOS), commonly manifesting with pronounced neuromuscular symptoms, fatigability and exercise intolerance. The subunits of the OXPHOS machinery are encoded by both the nuclear and mitochondrial DNA and pathogenic mutations in either genome can affect OXPHOS capacity, often presenting with isolated or combined mitochondrial complex deficiencies. Complex I deficiency (NADH:ubiquinone oxidoreductase) is the most prevalent OXPHOS disorder and is found either in isolation or combined with deficiencies of the other complexes (3). Treatment options in complex I deficiencies are currently limited, despite extensive research on a variety of approaches, and successes are often anecdotic and not applicable to all patients. Clinical improvements as a result of high-fat intake with or without carbohydrate restriction have been reported for some of the complex I deficient patients and have led to the prescription of high-fat and ketogenic diets to patients with complex I, but also with pyruvate dehydrogenase complex deficiencies, Leigh syndrome, epilepsy and Parkinson disease (4-10). The resulting increase in plasma fatty acids and deprivation of carbohydrates is expected to cause a shift from a glycolytic energy metabolism to the beta-oxidation of fatty acids, which is accompanied by the production of ketone bodies in the liver mitochondria (11-13). Ketone bodies (3-beta-hydroxybutyrate, acetoacetate, and acetone) are shortchain organic acids that can freely diffuse across cell membranes and were suggested to serve as an alternative energy substrate to glucose for the brain $(14,15)$. The ketogenic diet was shown to have beneficial effects for patients with pyruvate dehydrogenase complex deficiency related brain pathology, especially in case of pharmaco-resistant epilepsy (4). Although different mechanisms of action, involving both ketone bodies and fatty acids, have been proposed (16-22), the precise mechanisms are not clear. Also for the treatment of mitochondrial myopathies due to complex I deficiency the therapeutic use of high-fat diets has been recommended, as supplementation of fatty acids via direct triacylglycerol infusion was shown to significantly improve exercise endurance in some patients (5). The latter observation was explained by an increase in mitochondrial beta-oxidation, which results in relatively higher $\mathrm{FADH}_{2}: \mathrm{NADH}^{+}+\mathrm{H}^{+}$ratios than the carbohydrate driven tricarboxylic acid cycle, thereby enabling bypassing of the complex I defect $(5,23,24)$. Yet, this mechanism cannot by itself explain why only some of the complex I deficient patients respond to highfat treatment, suggesting that these treatments influence the OXPHOS system, at least in part, in a gene- or protein-specific manner $(25,26)$. 
Despite the clinical application of high-fat diets, it is yet unknown which genetic complex I deficiencies could benefit from these diets. In this paper, we characterize the gene defect in a patient with complex I deficiency using whole-exome sequencing, who clinically improved on a high-fat diet. We tested the in vitro responsiveness to the saturated fatty acid palmitic acid of this patient and other patients with a genetically characterized complex I deficiency. This simple in vitro assay could be used in a clinical setting to select the patients who could benefit from this treatment.

\section{Material and Methods}

\section{Patient}

A Dutch female patient, who has a healthy younger brother and gave birth to a healthy child, was subject to HPLC based metabolite profiling on blood-plasma and urine to reveal metabolic abnormalities. A Quadriceps muscle biopsy was taken to spectrophotometrically measure OXPHOS complex activities for complex I, II+III, IV and V, normalized to citrate synthase activity, according to a method described by Sgobbo et al. (27). Muscle histochemistry was performed by staining the succinate dehydrogenase (SDH) and cytochrome oxidase (COX) complexes and electron microscopy was performed to identify abnormalities in mitochondrial morphology and quantity. A skin biopsy was taken from the TMEM126B patient and dermal fibroblast were isolated in a similar manner (as described below) as previously executed for the five other patients with complex I deficiency (NDUFS7, NDUFAF5, ACAD9, NDUFA12, NDUFV2 defective fibroblasts), who were all biochemically and genetically diagnosed at the Maastricht University Medical Centre (MUMC+).

\section{Dermal fibroblast sampling, isolation and culturing}

Primary dermal fibroblasts from the patient were obtained by taking a 3-5mm punch biopsy from the arm, which was cut into 18 smaller pieces and 3 biopsy pieces were transferred to each gelatin pre-coated 6-well containing Dulbecco's Modified Eagle Medium (DMEM, 25mM glucose) supplemented with $20 \%$ fetal bovine serum (FBS). When reaching confluency, fibroblasts were trypsinized and transferred to a T75 flask, and, after 3 passages, immuno-stained to check HSP-47 positivity. During later passages fibroblasts were cultured in DMEM (25mM glucose) supplemented with $10 \%$ FBS and $0.2 \%$ Penicillin-Streptomycin. 


\section{Dietary studies}

The patient's (TMEM126B patient) muscle endurance was examined by performing a bicycle test at $15 \%$ of the maximal capacity ( $15 \%$ of Wmax), based on the patient's usual diet in which $34,5 \%$ of the energy was derived from fat and $49,4 \%$ from carbo-hydrates. Magnetic Resonance Phosphor Imaging (31P MRS) of the muscle was performed to measure muscle recovery. A dietary intervention study was performed, where the patient was first prescribed to consume a high-carbohydrate diet for 3 weeks, where $25 \%$ of the energy derived from fat, and subsequently a high fat-diet, where $55 \%$ of the energy was derived from fat intake, to study the effects of dietary intake on muscle endurance, measured by the bicycle test at $15 \%$ of Wmax. Additionally, differences in muscle endurance and strength were examined with direct substrate infusion, comparing intra-lipid infusion $(3,7 \mathrm{mg} / \mathrm{kg} / \mathrm{min}$.) to glucose infusion (10 $\mathrm{mg} / \mathrm{kg} / \mathrm{min}$.), and measuring muscle strength at different body parts with intervals of relaxation.

\section{Whole-exome sequencing}

Blood DNA was fragmented and exons were captured using the Agilent SureSelect version 4, exome enrichment kit, including UTRs (Agilent Technologies, Santa Clara, CA, USA). Sequencing was performed on an Illumina HiSeq2000 platform, using a 2x100 bp paired end setting (Illumina, San Diego, CA, USA). Bcl2fastq 1.8.4 allowed base-calling and demultiplexing; BWA 0.5.9 was used for read alignment against human reference genome hg19. Duplicate reads were removed by Picard software suite 1.77 (Broad Institute); variant calling was performed with GATK 2.1-8 (Broad Institute). An in-house data analysis pipeline extracted database information on annotated variants according to the UCSC RefGene track; dbSNP137 and dbNSFP v2.0. Exome data were filtered for homozygous and heterozygous variants with allele frequencies lower than 1\% (dbSNP137) and a coverage of $>10$ reads, consisting of non-synonymous substitutions, INDELs (in-frame and frameshift), nonsense mutations and splice-variants. Non-annotated variants were maintained, unless allele frequencies exceeded $5 \%$ in our in-house patient database. Missense variants in moderately to highly conserved domains were maintained. Pathogenicity of non-synonymous missense mutation was estimated by Polymorphism Phenotyping-2 (PolyPhen-2), Sorting Intolerant From Tolerant (SIFT) and PROVEAN.

\section{Sanger sequencing and QPCR}

Mutation segregation analysis was performed on blood DNA from the healthy mother and index patient. The father was not available for further analysis. Primers were designed 
to cover the exon 5 missense mutation in TMEM126B (transmembrane protein 126B, NC_000011.10) (Supplementary Table S2). The TMEM126B nDNA copy number was quantified by the $7900 \mathrm{HT}$ Fast Real-Time PCR System, normalizing to nuclear B2M (nuclear gene beta-2-microglobulin, NC_000015.10). Primers were designed in exon 1, 2 and 5 (Supplementary Table S3) and amplification was performed using Sensimix Sybr Hi-Rox (Bioline, Taunton, MA, USA).

\section{Blue native-polyacrylamide gel electrophoresis}

Mitoplasts were isolated from fibroblasts of the index patient, control 1 (C1) fibroblasts, which were Normal Human Dermal Fibroblasts (NHDF), control 2 (C2) fibroblasts derived from a single healthy individual, and an NDUFA9 mutant as previously described (28). $25 \mu \mathrm{g}$ mitoplast fraction was loaded on a $4-16 \%$ polyacrylamide gradient gel (Invitrogen), after quantification by Qubit Protein Assay Kit (Thermo Fisher Scientific, Waltham, MA, USA). Western blot analysis was performed by hybridization with monoclonal antibodies for complex I subunit NDUFA5, NDUFB8 and complex II (SDHA) as a protein loading control (Mitosciences, Eugene, OR, USA).

\section{Cloning and lentiviral complementation}

TMEM126B cDNA (isoform A, NM_018480.4) was cloned into a $3^{\text {rd }}$ generation pUltraChili lentiviral backbone vector, containing a tomato-red reporter gene for validating viral transduction efficiency (Addgene, Malcolm Moore Lab). The p.G212V mutation was introduced by side directed mutagenesis according to the QuikChange II Site-Directed Mutagenesis Kit (Agilent Technologies). Transfection of HEK293FT viral producing cells was accomplished by combining transIT (Mirus Bio), $3^{\text {rd }}$ generation packaging and envelope plasmids (Rev, Gag and Pol, VSV-G) and the backbone expression vector. $48 \mathrm{~h}$ post transfection, media containing viral particles were collected and filtered $(0.45 \mu \mathrm{m})$, subsequently polybrene was added $(8 \mathrm{ug} / \mathrm{ml})$. Patient fibroblast were seeded at $30 \%$ confluency and transduced for $24 \mathrm{~h}$, resulting in a transduction efficiency of $>90 \%$.

\section{Seahorse analysis}

Patient (TMEM126B) fibroblasts, control 1 (C1) fibroblasts, which were Normal Human Dermal Fibroblasts (NHDF), and control 2 (C2) fibroblasts, deriving from a single healthy individual, were seeded at 22,000 cells per well, using 12 wells per condition, and attached overnight in standard DMEM (25mM glucose) supplemented with FBS (10\%). 1h prior to assay, media was replaced with Seahorse assay media containing $10 \mathrm{mM}$ glucose, $2 \mathrm{mM}$ 
glutamine, $1 \mathrm{mM}$ pyruvate, $10 \% \mathrm{FBS}$, and oxygen consumption rates (OCR) were measured using the Seahorse XF Cell Mito Stress Test Kit and the Seahorse XFe96 device (Seahorse Biosciences, North Billerica, MA, USA) according to the manufacturer's protocol. To test the effect of free fatty acids (FFAs) on the mitochondrial respiration in patient (TMEM126B, NDUFS7, NDUFV2, NDUFA12, NDUFAF5) and control (C1) fibroblasts, we seeded 12,000 fibroblasts per well, using 8 replicate wells per condition, in a Seahorse XF 96-well plate. Cells attached overnight in normal DMEM $(25 \mathrm{mM})$ growth media, supplemented with $10 \%$ FBS. Prior to assay, fibroblasts were incubated for $16 \mathrm{~h}$ in DMEM with glucose (25mM), but with limited FBS (1\%), where either 500 $\mathrm{MM}$ BSA-conjugated palmitic acid or oleic acid was added to the media of the treated conditions and unbound BSA was added to the untreated conditions. FFAs need to be BSA-conjugated (ratio FFA : BSA=6:1) in order to be taken up by the cells. $1 \mathrm{~h}$ before assay, media was replaced with Seahorse assay media containing $10 \mathrm{mM}$ glucose, $2 \mathrm{mM}$ glutamine, $1 \mathrm{mM}$ pyruvate, $1 \% \mathrm{FBS}$, and oxygen consumption rates (OCR) were measured using the Mitostress kit according to the manufacturer's manual. Administration of oligomycin $(2.0 \mu \mathrm{M})$ blocked complex $\mathrm{V}$ activity, and was used as a measure for ATP production (start resp. - oligomycin resp.). Uncoupling agent FCCP $(1.0 \mu \mathrm{M})$ stimulated cells to their maximal respiration level and was used together with antimycin A/rotenone $(0.5$ $\mu \mathrm{M})$, blocking complete OXPHOS respiration via complex I and III, as a measure for maximal respiratory capacity (FCCP resp. - antimycin A/rotenone resp.). Furthermore, OXPHOS spare capacity (FCCP resp.- start resp.) and proton leak (oligmycin resp. - antimycin A/rotenone resp.) were determined. OCR was measured at 4 time points after compound administration, where the $4^{\text {th }}$ measurement was used to calculate statistical significance $(P<0.05)$. To correct for differences in proliferation rate, OCR's were normalized to control according to protein quantity.

\section{Statistical analysis}

Statistical significance was calculated using a two-sample t-test, one-tailed, equal variance. A 95\% confidence interval and alpha-level 0.05 was applied.

\section{Results}

\section{Patient clinic and OXPHOS complex I activity}

A female from a non-consanguineous family had a motor development delay from birth onwards, while mental milestones were reached in time. Muscle weakness and cramps in the legs developed and she had difficulties with walking. The muscle weakness could 
disappear after resting for two hours. Exercise intolerance persisted, and at 14 years of age metabolic investigations indicated elevated levels of lactic acid (2.8 - $5 \mathrm{mmol} / \mathrm{l})$. Alanine and pyruvic acid levels were normal. Muscle histochemistry showed an increased intensity and linear staining of succinate dehydrogenase (SDH) and cytochrome oxidase (COX) in the subsarcolemmal regions. Furthermore, ragged red fibers were seen in approximately $40 \%$ of the muscle tissue. Electron microscopy indicated elongated, enlarged and abnormally shaped mitochondria (subsarcolemmal) and dispersed dense bodies with abnormal cristae and cristalline inclusions (Supplementary Figure S1). Complex I activity of the patient was approximately $20 \%$ of control muscle (Table 1 ) and increased activities were measured for $\mathrm{CIV}$ and $\mathrm{CV}$. For the last ten years the patient remained easily fatigued.

Table 1: OXPHOS complex activities in patient muscle biopsy

\begin{tabular}{crccc}
\hline OXPHOS complex & \multicolumn{2}{c}{ Activity } & control SEM & $\%$ as to control SEM \\
\hline I & 0.76 & $(\mu \mathrm{mol} \mathrm{NADH} / \mathrm{min} / \mathrm{g})$ & $3.66 \pm 0.22$ & $21 \%(\downarrow)$ \\
II & n.d. & $(\mu \mathrm{mol} \mathrm{INT} / \mathrm{min} / \mathrm{g})$ & $723 \pm 40$ & n.d. \\
II+III & 7.43 & $(\mu \mathrm{mol} \mathrm{cyt} \mathrm{c/min} / \mathrm{g})$ & $5.20 \pm 0.32$ & $143 \%(\uparrow)$ \\
IV & \multicolumn{2}{c}{$195.0 \quad(\mathrm{k} / \mathrm{min} / \mathrm{g})$} & $94.6 \pm 3.5$ & $206 \%(\uparrow)$ \\
V & \multicolumn{2}{c}{$31.20(\mu \mathrm{mol} \mathrm{Pi} / \mathrm{min} / \mathrm{g})$} & $11.90 \pm 0.83$ & $262 \%(\uparrow)$ \\
\hline
\end{tabular}

\section{Dietary studies}

Bicycle endurance testing (15\% of $W_{\max }$ ) on the patient's (TMEM126B patient) usual diet indicated that muscle endurance was far below normal levels, where the test persisted 60 min. with a mean oxygen consumption $\left(\mathrm{VO}_{2 \text { mean }}\right)$ of $7,45 \mathrm{ml} / \mathrm{kg} / \mathrm{min}$. Also the maximal exertion $\left(\mathrm{W}_{\max }: 60 \mathrm{~W}\right)$ and maximal oxygen consumption levels $\left(\mathrm{VO}_{2 \max }: 13,0 \mathrm{ml} / \mathrm{kg} / \mathrm{min}\right.$.) were below normal level and Magnetic Resonance Phosphor Imaging (31P MRS) of the muscle showed delayed recovery of the phosphocreatine levels after exercise. Dietary intervention studies were performed to examine the effect of a high-carbohydrate diet ( $25 \%$ of energy from fat) versus a high fat-diet ( $55 \%$ of energy from fat). During the 3 weeks of carbohydrate diet, the patient felt increasingly exhausted and activities of daily living were more difficult to perform than with her usual diet. Exercise testing showed that the patient's bicycle endurance was $53 \%$ prolonged after the high-fat diet, compared to the carbo-hydrate diet (95min. versus $62 \mathrm{~min}$.), where the mean oxygen consumption was higher after the high-fat diet $\left(\mathrm{VO}_{2 \text { mean }}\right.$ : 8,51 versus $6,82 \mathrm{ml} / \mathrm{kg} / \mathrm{min}$.). No differences were measured with the maximal exertion test $\left(W_{\max }\right)$, although oxygen consumption was higher after the high-fat diet. Differences in endurance measured after direct substrate infusion showed that the patient sustained the bicycle test $38 \%$ longer ( $90 \mathrm{~min}$. versus $65 \mathrm{~min}$.) on intra-lipid infusion compared to glucose infusion. The mean oxygen consumption $\left(\mathrm{VO}_{2 \text { mean }}\right)$ was higher with lipid infusion than glucose infusion $(7,45 \mathrm{ml} / \mathrm{kg} / \mathrm{min}$. versus $5,09 \mathrm{ml} / \mathrm{kg} / \mathrm{min}$.). Also in muscle strength, which 
was measured at different body parts, lipid-infusion seemed to have an advantage in terms of strength of the lower extremities, with less decline between subsequent measurements. More subjective, the patient stated that she felt physically better when consuming a high-fat diet (Supplementary Table S1).

\section{Whole-exome sequencing (WES)}

WES analysis revealed a homozygous c.635G $>T$ missense mutation in the TMEM126B gene, a known complex I assembly factor (NM_018480.4:c.635G>T, chr: 11, g.85347215). The resulting p.G212V amino-acid substitution is located in a transmembrane region at a position that is highly conserved in mammals (Nextprot: NX_Q8IUX1). In vertebrates, the conservation of Glycine, Serine and Alanine at p.212 (belonging to a class of small size amino acids) suggested structural constraints on the size of the amino acid, which is violated by substitution with a larger valine residue. In silico pathogenicity predictions assigned highly damaging properties to the mutation at protein level (SIFT: 0.002, PROVEAN: -6.2, PF: 0.99). Mutation segregation analysis confirmed a recessive inheritance pattern as the mother was heterozygous carrier (Supplementary Figure S2). No DNA was available from the father and healthy brother, therefore the possibility of a hemizygous mutation was excluded by TMEM126B copy-number analysis (Supplementary Figure S3). The p.G212V mutation has an allele frequency of 0.1-0.2\% (dbSNP: rs141542003) in dbSNP and ExAC databases.

\section{Complex I assembly and lentiviral complementation}

The effect of the TMEM126B mutation on complex I integrity was studied by Blue-Native PAGE electrophoresis. Mitochondrial fractions were isolated from fibroblasts of the patient (TMEM126B patient), two controls (control 1 (C1) and control 2 (C2)) and an NDUFA9 mutant as a negative control. Antibodies against the subcomplex $\mid \alpha(C x \mid \alpha)$ subunit NDUFA5 were used to detect mature complex I and assembly intermediates containing this peripheral arm module ( $Q$ module) (29-31). A drastic loss of mature complex I and accumulation of the $315 \mathrm{kDa}$ intermediate was observed, indicating impaired complex I assembly in the patient (Figure $1 \mathrm{~A})$. Antibodies against the subcomplex $I \beta(C x \mid \beta)$ subunit NDUFB8 were used to detect assembly stages of the membrane arm of complex I (P module) $(30,32)$, and showed normal quantities of NDUFB8 in the mature complex I (Figure 1B). Complex II (SDHA) was used as a protein loading control. To provide evidence that defects in TMEM126B cause incomplete assembly of the peripheral arm of complex I, patient fibroblasts were rescued by lentiviral transduction with wild-type TMEM126B (isoform A, NM_018480.4). Blue-Native PAGE and immunoblotting with anti-NDUFA5 indicated that the amount of fully assembled complex I in patient fibroblasts was largely restored upon complementation (Figure 1C). 
A

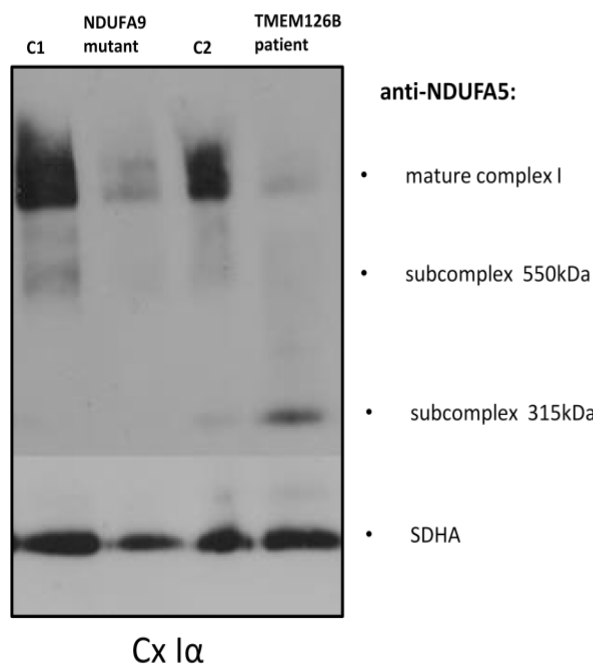

C

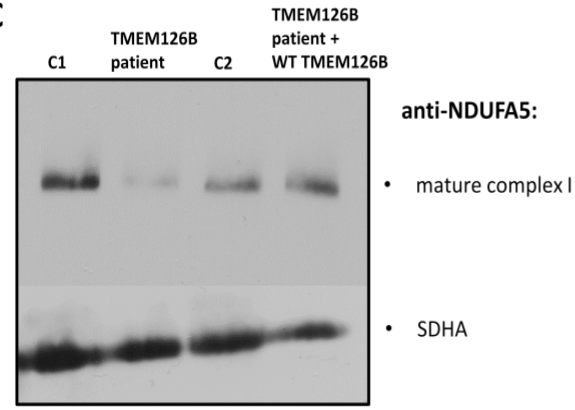

B

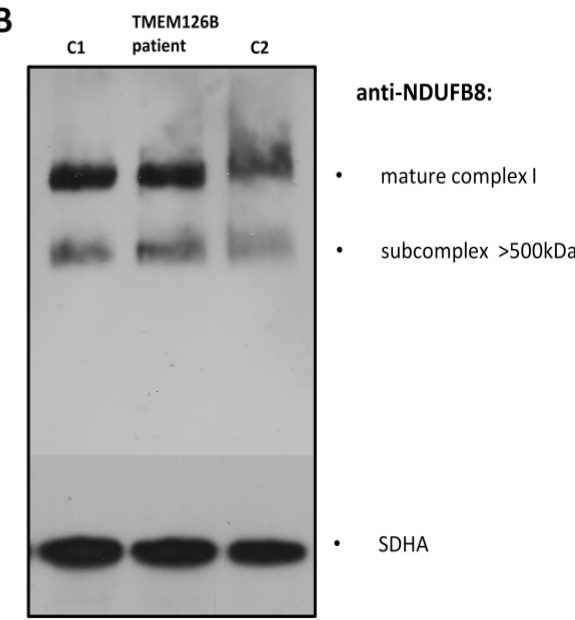

$C x \mid \beta$

$\mathrm{Cx} \mid \alpha$

Figure 1: Blue-Native PAGE on fibroblasts of the patient (TMEM126B patient), 2 controls (control 1 (C1), control 2 (C2)) and NDUFA9 mutant. (A) Anti-bodies against NDUFA5 were used to detect mature complex I and assembly intermediates containing the $Q$ module of the peripheral arm of complex I ( $C X$ Ia). A loss of mature complex I and accumulation of the 315kDa intermediate in the patient indicated impaired complex I assembly. (B) NDUFB8 antibodies detected assembly stages of the membrane arm of complex I (CX IB), which showed normal NDUFB8 quantities in mature complex I, suggesting that assembly of the membrane arm (P-module) succeeded despite missing parts of the peripheral matrix arm ((A) and (B) are the representatives of 2 replicates). (C) Patient fibroblasts (TMEM126B patient) were lentiviral transduced with wild-type TMEM126B (TMEM126B patient + WT TMEM126B) and showed a recovery of the amount of mature complex 1 when compared to healthy control fibroblast $(\mathrm{C} 1+\mathrm{C} 2)((\mathrm{C})$ consists of a single experiment). 


\section{Mitochondrial respiration and fatty-acid treatment}

A mitochondrial stress test was performed to examine OXPHOS capacity in patient fibroblasts. Oxygen consumption rate (OCR) measurements indicated decreased ATP production and decreased maximum respiratory capacity in patient fibroblast compared to control lines (C1 and C2) (Supplementary Figure S4A). As indicated by the extracellular acidification rates (ECAR), patient fibroblasts were more dependent on glycolytic metabolism than control fibroblasts (Supplementary Figure S4B). Respiratory findings therefore corresponded with the observed complex I assembly defect in the patient's fibroblasts. To test if unsaturated or saturated fatty acids have an effect on OXPHOS capacity, patient fibroblasts were treated with $500 \mu \mathrm{M}$ oleic acid or palmitic acid, respectively, for $16 \mathrm{~h}$ prior to measuring the oxygen consumption under different mitochondrial stress conditions. Whereas oleic acid did not have any significant effect on the oxygen consumption rate (OCR) in patient or control cells (C1) (Supplementary Figure S5), pre-incubation with palmitic acid resulted in a significant increase of $25 \%$ in maximal respiratory capacity in TMEM126B patient fibroblasts, from $63 \%$ to $88 \%$ of the untreated control level (Figure $2 \mathrm{~A}$ and $2 \mathrm{~B}$ ). In control fibroblasts, palmitic acid treatment significantly increased maximal respiratory capacity with $21 \%$. This increase in maximal respiration was accompanied by an increase in respiratory spare capacity of $45 \%$ and $67 \%$ in control and patient cells, respectively (Figure 2C). ATP production in patient fibroblasts was $63 \%$ of the untreated control condition and increased $10 \%$ as a result of palmitic acid treatment, however this was not statistically significant (Figure 2D). Proton leakage was not significantly changed, where basal respiration due to non-coupled ATP production was never higher than $17 \%$ (Figure 2E).

To test if the increase in maximal respiratory capacity after palmitic acid treatment in control and TMEM126B patient fibroblasts (p.G212V) was related to complex I function in general, we performed similar respiratory measurements in patient fibroblasts with a variety of complex I gene defects, all patients being $\mathrm{Cl}$ deficient in muscle biopsy, but not all of them with a significant respiratory deficiency in fibroblasts (Figure 3). This included patients with mutations in the subunits NDUFS7 (p.V122M), NDUFA12 (c.83dup, frameshift), NDUFV2 (p.A183T) and assembly factors ACAD9 (p.R532W) and NDUFAF5 (p.L159F). As indicated in Figure 3 and Supplementary Figure S6, fibroblast with pathogenic mutations in NDUFS7 and NDUFAF5 showed a significant increase of $32 \%$ (from $63 \%$ to $83 \%$ of untreated control) and $22 \%$ (from $107 \%$ to $131 \%$ of untreated control), respectively, in maximal respiratory capacity upon palmitic acid treatment. No significant influence of palmitic acid on maximal respiration was measured in the NDUFA12 (71\% of control), NDUFV2 (75\% of control) and ACAD9 (70\% of control) fibroblasts. 


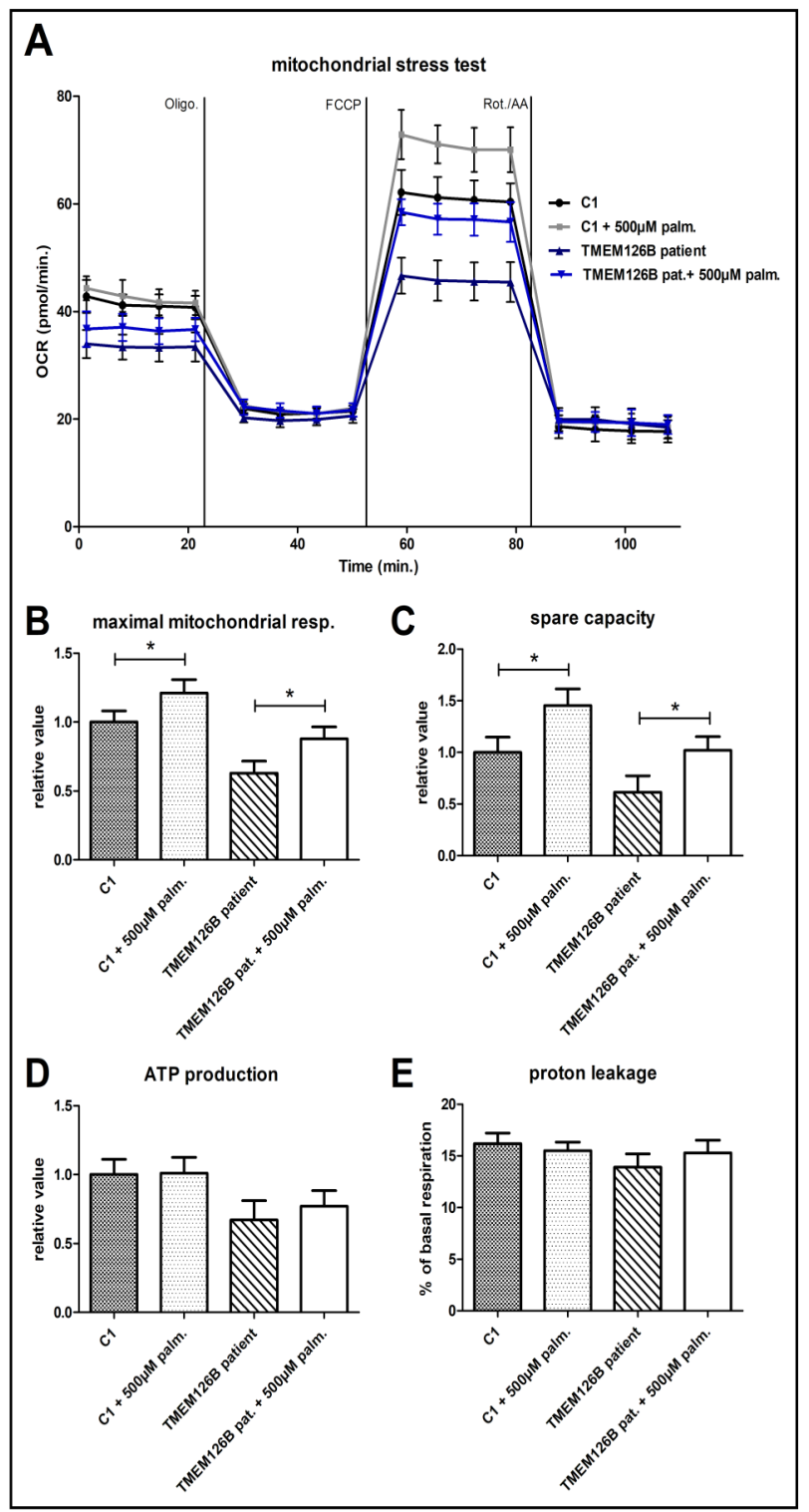

Figure 2: Mitochondrial stress test in TMEM126B patient fibroblasts (A) Oxygen consumption rates (pMoles/min) measured in patient and healthy control fibroblasts (C1) after 16h treatment with $500 \mu \mathrm{M}$ palmitic acid. (B) Maximal respiratory capacity in both control and patient fibroblasts increased with $21 \%$ and $25 \%$, respectively, upon treatment with palmitic acid, where respiration in the TMEM126B patient restored from $63 \%$ to $88 \%$ of the control level. (C) The measured increase in maximal respiration resulted in a $45 \%$ and $67 \%$ increase in respiratory spare capacity in control and patient cells, respectively. (D) ATP production in patient fibroblasts was $63 \%$ of the untreated control level and was not significantly altered by palmitic acid treatment. E) Also proton leakage was not significantly changed, where basal respiration due to non-coupled ATP production was never higher than $17 \%$. 


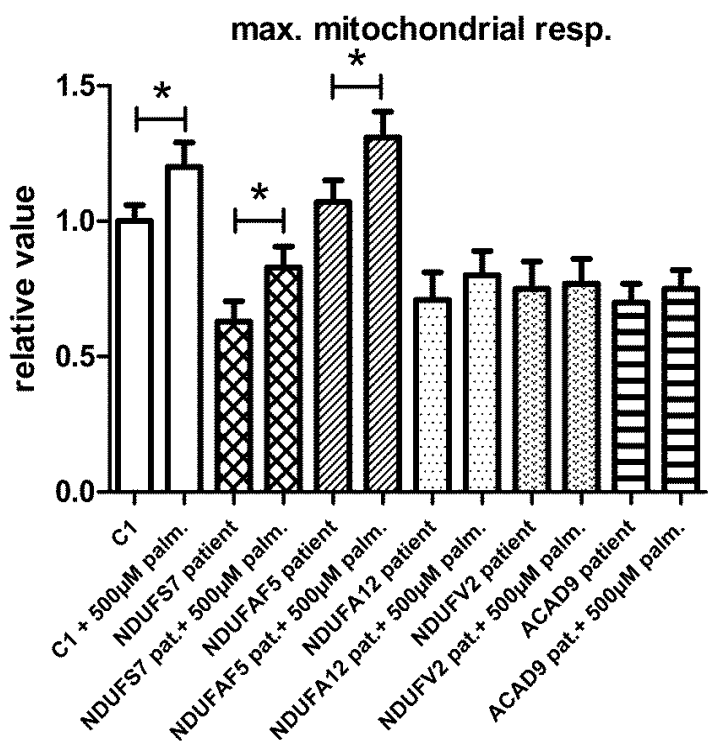

Figure 3: The effect of palmitic acid on the maximal respiratory capacity in fibroblasts. Control (C1) and patient fibroblasts with defects in the complex I subunit and assembly factor genes NDUFS7 (p.V122M), NDUFAF5 (p.L159F), NDUFA12 (c.83dup, frameshift), NDUFV2 (p.A183T), and ACAD9 (p.R532W) were treated with palmitic acid prior to seahorse analysis. NDUFS7 and NDUFAF5 cells were responsive to palmitic acid as a significant increase of respectively $32 \%$ and $22 \%$ in maximal respiratory capacity was measured. No significant changes in maximal respiration were measured in NDUFA12, NDUFV2 and ACAD9 defective fibroblasts.

\section{Discussion}

Dietary studies in a complex I deficient patient with exercise intolerance indicated beneficial effects of a high-fat diet on the patient's muscle endurance. WES analysis was performed to characterize the genetic defect and identified a highly damaging, homozygous p.G212V substitution in the transmembrane region of TMEM126B (NM_018480.4:c.635G>T). Our data show that the p.G212V mutation in TMEM126B causes an accumulation of the $315 \mathrm{kDa}$ complex, containing the peripheral arm subunit NDUFA5 ( $C x \mid \alpha)$, possibly via interrupted merging of the $315 \mathrm{kDa}$ and $370 \mathrm{kDa}$ subcomplexes (Figure 4). This resulted in a drastic loss of mature complex I in patient fibroblasts. The resulting complex I deficiency could be rescued by wild-type TMEM126B, confirming causality. Last year, the first patients with TMEM126B defects were identified, all carrying the p.G212V mutation in hetero- or homozygous fashion $(33,34)$, possibly due to the relatively high allele frequency in the European-American population (0.1-0.2\%). Our data show that biochemical and clinical differences exist among the homozygous p.G212V patients (Table 2). In line with the complex I deficiency described for subject 6 of the previously reported cases (33), we observed a respiratory deficiency in 
the patient's fibroblasts. In contrast, the second case, subject 1, showed a muscle specific respiratory deficiency. Clinical symptoms in our patient were similar to subject 1 , as both manifested with mild exercise intolerance (Table 2). In contrast, the other reported case suffered from more severe, heterogeneous symptoms, involving cardiomyopathy, renal failure and growth deficits. Yet, it is most likely that the p.G212V substitution explains a relatively mild phenotype, as all other reported TMEM126B patients were described to suffer mainly from exercise intolerance, even in combination with more harmful nonsense mutations. Most likely, in our opinion, the TMEM126B mutation by itself cannot explain the extended phenotype of the severely affected patient.

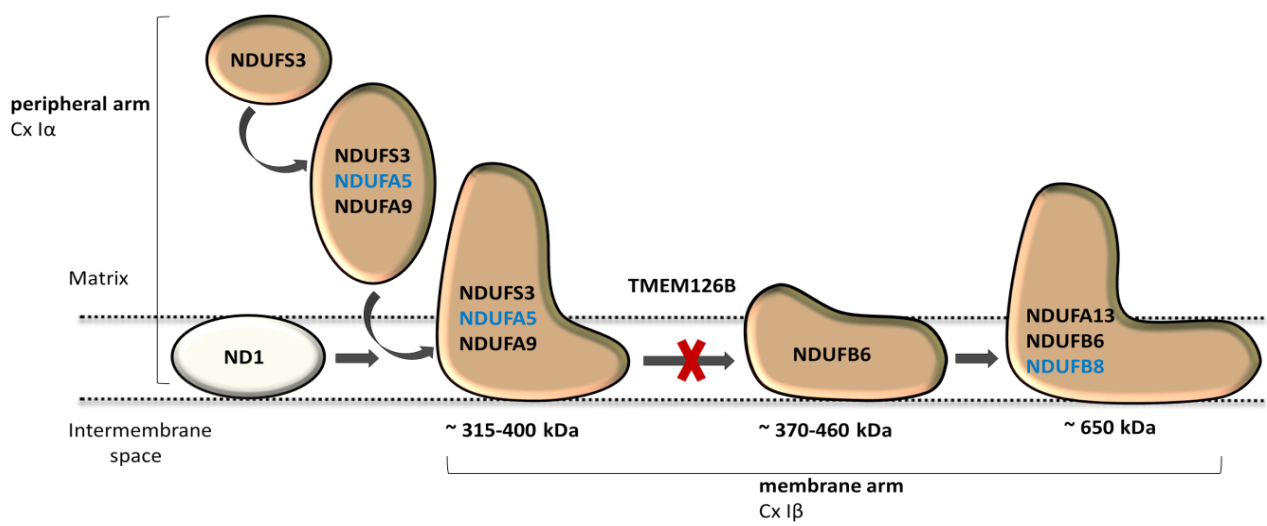

Figure 4: TMEM126B functions as an early complex I assembly factor. TMEM126B is required for the joining of the $\sim 315 \mathrm{kDa}$ and $\sim 370 \mathrm{kDa}$ assembly intermediates (previously estimated $\sim 400 \mathrm{kDa}$ and $\sim 460 \mathrm{kDa}$, respectively). BN-Page on TMEM126B defective patient fibroblasts indicated accumulation of NDUFA5 (Q-module) in the $315 k D a$ intermediate and a drastic decrease in complex l, indicating that part of the peripheral arm was missing. Membrane arm subunit NDUFB8 ( $N$-module) was present in normal quantities in mature complex I, indicating that assembly of the complex I membrane arm succeeded despite missing parts of the peripheral arm. 


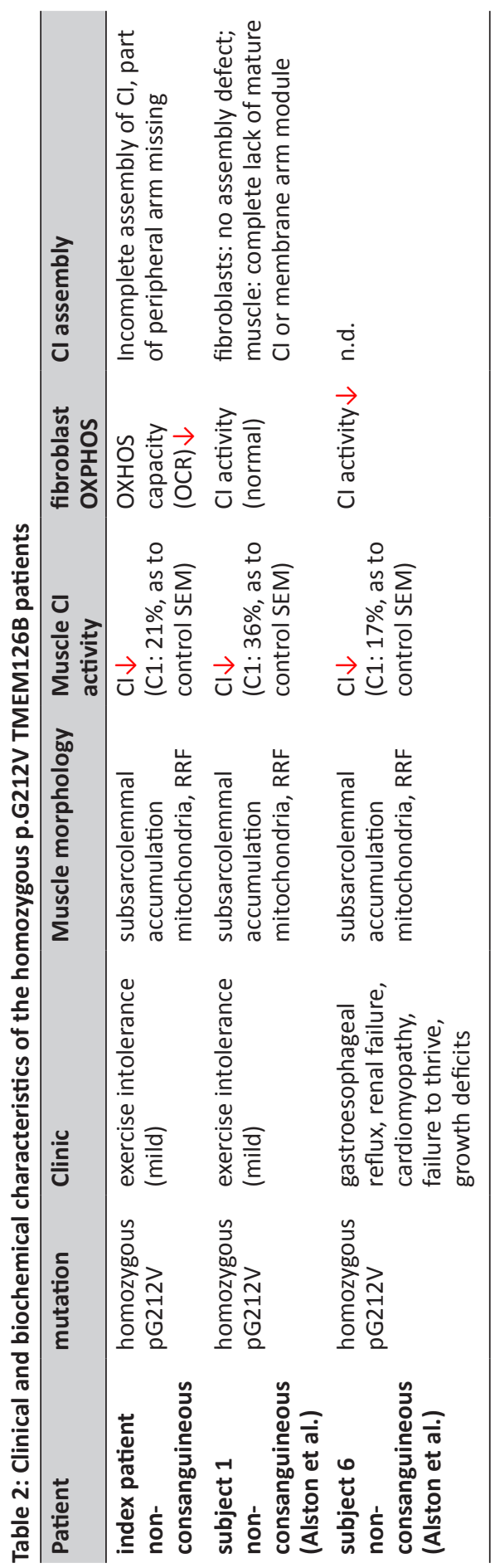


Considerable differences in complex I assembly were observed in TMEM126B patients (Table 3). Where our data indicated that an incomplete constitution of complex I affected mitochondrial respiration in both muscle and fibroblasts, a previously reported homozygous p.G212V patient did not show an assembly defect in fibroblasts at all. Subunits from both the early $\sim 315 \mathrm{kDa}$ peripheral arm intermediate (NDUFA9, NDUFS3; Q-module) and the 370kDa membrane arm (NDUFB6; P-module) were present in mature complex I in normal quantities. In contrast, complex I in muscle tissue showed an almost complete lack of the membrane arm subunit NDUFB8 ( $650 \mathrm{kDa}$ intermediate), likely indicating a decrease in the total amount of mature complex I (Tabel 3, Figure 4). The p.G212V mutation in combination with more harmful nonsense and splice mutations was shown to cause a decrease in total amount of mature complex I, as subunits of the peripheral arm (NDUFS3, NDUFA9) and membrane arm were significantly decreased (NDUFB8, NDUFA13, NDUFB6) $(32,35)$. This is striking as the severity of the assembly defect is apparently not defined by the mildest mutation. As shown in Figure 4, the current complex I assembly model is suggested to start with the joining of a $\sim 315 \mathrm{kDa}$ subcomplex, containing the peripheral arm subunit NDUFA5 (Q-module, $\mathrm{Cx} \mid \alpha$ ), and a $\sim 370 \mathrm{kDa}$ subcomplex, containing the membrane arm subunit NDUFB8 (N-module, Cx I $\beta$ ) (previously estimated $\sim 400 \mathrm{kDa}$ and $\sim 460 \mathrm{kDa}$, respectively) (30-32, 36). The MCIA complex, containing TMEM126B, was found to be associated with the $\sim 370 \mathrm{kDa}$ assembly intermediate. siRNA-knockdown experiments in human 143B cells confirmed a role for TMEM126B as an early extrinsic assembly factor as a significant loss of the peripheral arm subunit NDUFA5 in complex I occurred together with an accumulation of the $\sim 315 \mathrm{kDa}$ subcomplex (31). Membrane arm subunit NDUFB8 revealed elevated levels of the $\sim 370 \mathrm{kDa}$ subcomplex and decreased amounts of the larger $\sim 550 \mathrm{kDa}$ intermediate, suggesting that TMEM126B is required for the joining of the $\sim 315 \mathrm{kDa}$ and $\sim 370 \mathrm{kDa}$ subcomplexes during early complex I assembly. Yet, as NDUFB8 was still abundant in mature complex I, it is likely that part of the membrane arm assembly succeeded. 
Palmitic acid responsive patients with an OXPHOS complex I defect

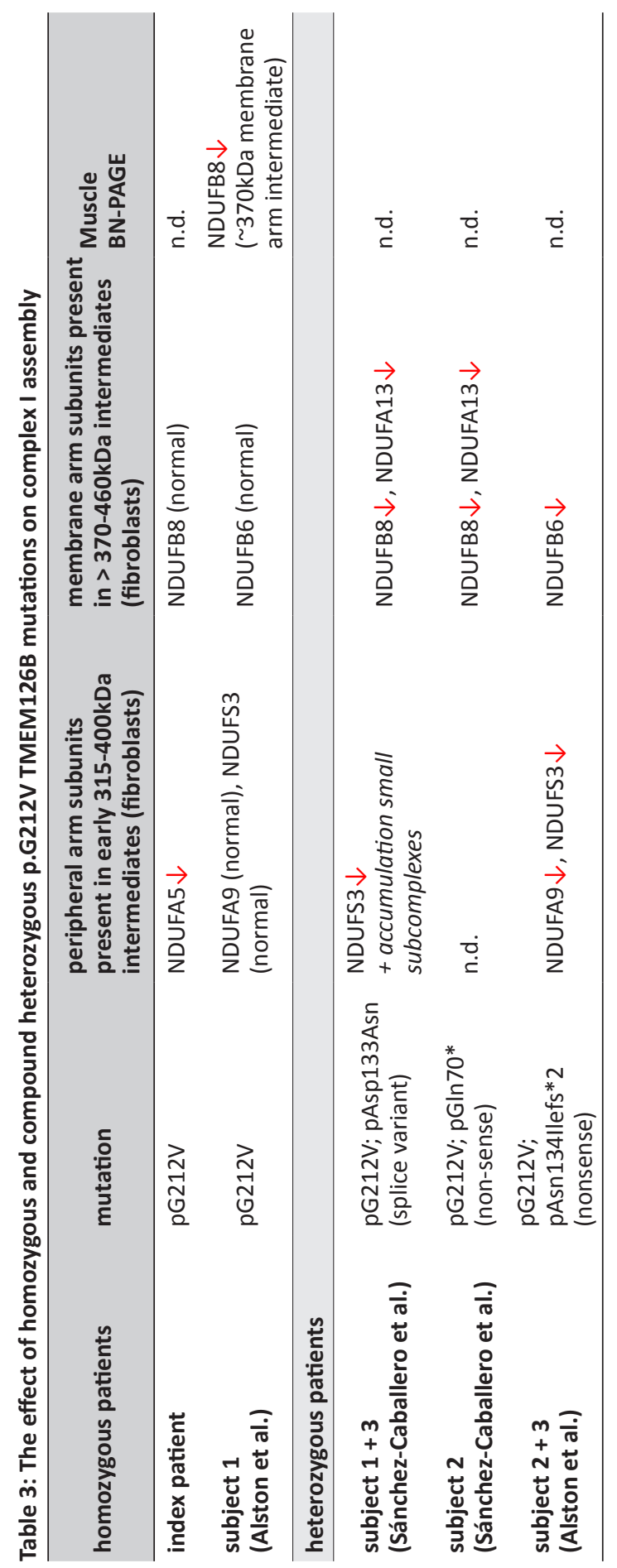


Our patient showed improved muscle endurance and strength on a high-fat diet compared to a high carbo-hydrate diet. For the clinical benefits of high-fat intake in complex I deficiency anecdotic reports exist and have led to the clinical use of different therapeutic high-fat diets and ketogenic diets (low in carbohydrates). Not all complex I deficient patients seem to benefit from such diet, but it is currently unclear which complex I gene defects respond to these therapies and how mitochondrial function is influenced by an increase in fatty-acids or ketone bodies. FFAs are an important source for mitochondrial energy production, but it has been difficult to elucidate their effect on mitochondrial function as contradictory effects have been reported in terms of mitochondrial oxygen consumption and toxicity (37-39). In line with mitochondrial respiration data from mouse podocytes, mouse neonatal myocytes and Normal Human Dermal Fibroblasts (NHDF), we measured a significant increase in oxygen consumption of healthy control fibroblasts (NHDF) due to palmitic acid treatment $(38,40,41)$. Maximal mitochondrial respiration capacity and spare capacity significantly increased with palmitic acid. Besides the maximal respiratory capacity, the spare mitochondrial respiratory capacity (or reserve capacity) has been reported as an important part of mitochondrial function, especially when cells are subject to stress, demanding increased levels of ATP to maintain cellular functions. Improvements in spare respiratory capacity can lead to increased ATP production to overcome stress and enhance cell viability, where complex II was suggested to be an important source of this reserve capacity $(40,42)$. As there have been indications that long-chain fatty acid can increase proton leak in liver mitochondria, possibly via mitochondrial uncoupling by the ATP/ADP and aspartate/glutamate antiporters, we have assessed whether this mito-toxic effect also occurred during our respiratory assay. We measured no significant increase in proton leakage due to palmitic acid treatment in the fibroblasts, which could otherwise have influenced the measured maximal respiratory levels $(39,43)$.

Our data show that, despite the severe complex I assembly defect, maximal mitochondrial respiration and spare capacity in TMEM126B defective patient cells significantly improved due to palmitic acid treatment (up to $88 \%$ of untreated control level), therewith supporting the dietary intervention studies in this patient. Interestingly, the unsaturated fatty acid oleic acid did not have any effect on mitochondrial respiration. The same assay was used for testing patient fibroblasts with different complex I defects, affecting both subunits and assembly factors, and showed that only part of the complex I defects are responsive to palmitic acid treatment, including NDUFS7 and NDUFAF5 defects. We demonstrate that this is an easy assay, which could be used as a personal medicine tool in the clinic to discriminate complex I deficient patients who might benefit from the intake of palmitic-acid, and those who could not (44). In line with the current knowledge on FFAs, we show that the effects strongly differ among the individual FFAs, as the unsaturated FFA oleic acid did not have 
any effect on mitochondrial respiration at all. Whereas in clinical practice, different diets are subsequently tested in the patient, without knowing the effective components, our cellline assay could overcome these limitations as a tool to identify responsiveness to specific FFAs (or other nutrients or drugs), thereby providing a personalized treatment approach. Although, therapeutic high-fat diets could be a possible way to increase the intake of palmitic acid in responsive patients, one should be aware that individual FFAs could behave differently as part of a complete diet (45).

It is difficult to fully explain mechanistically the differences in reaction by the data available. A proposed mechanism for FFAs on mitochondrial function involved the induction of the PPARy pathway. The saturated FFAs deconoic acid and palmitic acid, but not the unsaturated FFA oleic acid, were previously shown to increase PPAR p protein expression or, as an agonist, stimulate its activity. PPARy can stimulate mitochondrial biogenesis and proliferation, and could therefore increase cellular respiratory capacity $(25,46,47)$. However, this would be a general effect, suggesting that this mechanism cannot explain the differences between the patients, because it would imply that all $\mathrm{Cl}$ deficient patients in our assay should have reacted to the treatment, independent of the assembly defect. An alternative explanation could be an increase in FFA beta-oxidation, which results in relatively higher $\mathrm{FADH}_{2}: \mathrm{NADH}$ ratio's (1:1) than glycolysis (1:3). As $\mathrm{FADH}_{2}$ can directly donate its electrons to complex II of the OXPHOS system, it could bypass the complex I defect, therewith improving mitochondrial respiration $(5,23,24)$. Although the latter mechanism might contribute to an increase in respiratory capacity, this cannot explain our observation that only part of the complex I defects responds to palmitic acid treatment (Table 4). Complex I specific effects have previously been reported for both FFA's and ketone bodies, where decanoic acid was shown to increase complex I activity in healthy neuronal cells (25), and ketone bodies were shown to alleviate mitochondrial dysfunction by increasing complex I activity in MELAS cells (neuronal cybrid cells with m.3243A>G) via restoration of the complex I assembly defect (26). Interestingly, we found genetic defects in assembly factors and subunit (NDUFAF5, NDUFS7 and TMEM126B) involved in early assembly of the peripheral arm of complex I responsive to palmitic acid (Table 4) $(32,36)$. Although ACAD9, which is functional in the same MCIA complex as TMEM126B, did not respond to the treatment, this protein is known to have another crucial function as a catalyzer in the fatty acid beta-oxidation (48), making its role more complicated. The non-responsive gene defects affecting the subunits NDUFA12 and NDUFV2 are incorporated at a later assembly stage (>800kDa) (32), suggesting a compensating role for FFA in the early assembly process. However, current data is still too limited to draw a definite conclusion on the mechanism and testing more complex I defects will be needed. 
Table 4: The effect of palmitic acid on respiratory capacity in patient fibroblasts with different $\mathrm{Cl}$ defects

\begin{tabular}{|c|c|c|c|c|}
\hline complex I defect & patient clinic & $\begin{array}{l}\text { M / F complex I } \\
\text { activity }\end{array}$ & $\begin{array}{l}\text { OXPHOS deficiency } \\
\text { on seahorse (F) }\end{array}$ & $\begin{array}{l}\text { significant effect of palmitic } \\
\text { acid on max. resp. }\end{array}$ \\
\hline healthy control (NHDF) & n.a. & normal & no & yes (20-21\% increase) \\
\hline $\begin{array}{l}\text { TMEM126B: p.G212V } \\
\text { (c.635G>T) substitution }\end{array}$ & $\begin{array}{l}\text { exercise } \\
\text { intolerance }\end{array}$ & $\mathrm{M}: \mathrm{Cl}=21 \%$ & yes & yes ( $25 \%$ increase) \\
\hline $\begin{array}{l}\text { NDUFS7: } p . V 122 M \\
\text { (c.364G>A) substitution }\end{array}$ & Leigh & $\begin{array}{l}\mathrm{M}: \mathrm{Cl}=37 \% \text { (mild } \\
\text { decrease } \mathrm{CIII} \text { ) }\end{array}$ & yes & yes (32\% increase) \\
\hline $\begin{array}{l}\text { NDUFAF5: p.Leu159Phe } \\
\text { (c.477A >C) substitution }\end{array}$ & Leigh & $\mathrm{M}: \mathrm{Cl}=32 \%$ & no & yes ( $22 \%$ increase) \\
\hline $\begin{array}{l}\text { NDUFA12: homozygous } \\
\text { c.83dup, frameshift }\end{array}$ & Leigh & $\begin{array}{l}\mathrm{M}: \mathrm{Cl}=16 \% \\
\mathrm{~F}: \mathrm{Cl}=47 \%\end{array}$ & yes & no \\
\hline $\begin{array}{l}\text { ACAD9: p.Arg532Tryp } \\
\text { (c.1594C>T) substitution }\end{array}$ & $\begin{array}{l}\text { exercise } \\
\text { intolerance, } \\
\text { fatigue }\end{array}$ & $\begin{array}{l}\mathrm{M}: \mathrm{Cl}=30 \%, \mathrm{~F}: \\
\mathrm{Cl}=50 \%\end{array}$ & yes & no \\
\hline $\begin{array}{l}\text { NDUFV2: } \text { p.Ala183Thr } \\
\text { (c.547G>A) substitution }\end{array}$ & $\begin{array}{l}\text { white matter } \\
\text { degeneration }\end{array}$ & $\mathrm{M}: \mathrm{Cl}=47 \%$ & yes & no \\
\hline
\end{tabular}

"blue" colored row= assembly factor defect, "grey" colored row= subunit defect, M=muscle, F=fibroblast

Our data shows at a biochemical level that palmitic acid intake, possibly also as part of a therapeutic high fat diet, can be beneficial for patients with a defect in assembly factor TMEM126B and that our cell line assay will be a useful approach for the selection of patients, who might potentially benefit from this therapeutic diet. It is obvious that this cell line assay could also be extended to test responsiveness of patients to other nutrients and drugs, using mitochondrial respiration as outcome measure.

\section{Acknowledgement}

This work was supported by the Prinses Beatrix Spierfonds, grant application no. W.OR11-24 and Stichting Metakids. The pUltra-Chili lentiviral vector was gratefully obtained from Malcolm Moore Lab (Addgene).

\section{Ethics approval and informed consent}

The study was performed on the fibroblast cell lines with informed consent of the participants on the use of rest material of routine clinical procedures for research, as approved by the local ethical committee of Maastricht University Medical Centre. Written informed consent, in accordance with the Declaration of Helsinki, was obtained from the TMEM126B patient. 


\section{References}

1. Skladal D, Halliday J, Thorburn DR. Minimum birth prevalence of mitochondrial respiratory chain disorders in children. Brain. 2003;126(Pt 8):1905-12.

2. Gorman GS, Schaefer AM, Ng Y, Gomez N, Blakely EL, Alston CL, et al. Prevalence of nuclear and mitochondrial DNA mutations related to adult mitochondrial disease. Ann Neurol. 2015;77(5):753-9.

3. McFarland R, Kirby DM, Fowler KJ, Ohtake A, Ryan MT, Amor DJ, et al. De novo mutations in the mitochondrial ND3 gene as a cause of infantile mitochondrial encephalopathy and complex I deficiency. Ann Neurol. 2004;55(1):58-64.

4. Sofou K, Dahlin M, Hallbook T, Lindefeldt M, Viggedal G, Darin N. Ketogenic diet in pyruvate dehydrogenase complex deficiency: short- and long-term outcomes. J Inherit Metab Dis. 2017;40(2):237-45.

5. Roef MJ, de Meer K, Reijngoud DJ, Straver HW, de Barse M, Kalhan SC, et al. Triacylglycerol infusion improves exercise endurance in patients with mitochondrial myopathy due to complex I deficiency. Am J Clin Nutr. 2002;75(2):237-44.

6. Kang HC, Lee YM, Kim HD, Lee JS, Slama A. Safe and effective use of the ketogenic diet in children with epilepsy and mitochondrial respiratory chain complex defects. Epilepsia. 2007;48(1):82-8.

7. Paleologou E, Ismayilova N, Kinali M. Use of the Ketogenic Diet to Treat Intractable Epilepsy in Mitochondrial Disorders. Journal of clinical medicine. 2017;6(6).

8. Rogovik AL, Goldman RD. Ketogenic diet for treatment of epilepsy. Canadian family physician Medecin de famille canadien. 2010;56(6):540-2.

9. Baranano KW, Hartman AL. The ketogenic diet: uses in epilepsy and other neurologic illnesses. Current treatment options in neurology. 2008;10(6):410-9.

10. Wijburg FA, Barth PG, Bindoff LA, Birch-Machin MA, van der Blij JF, Ruitenbeek W, et al. Leigh syndrome associated with a deficiency of the pyruvate dehydrogenase complex: results of treatment with a ketogenic diet. Neuropediatrics. 1992;23(3):147-52.

11. Paoli A, Bosco G, Camporesi EM, Mangar D. Ketosis, ketogenic diet and food intake control: a complex relationship. Front Psychol. 2015;6:27.

12. Peters SJ, Leblanc PJ. Metabolic aspects of low carbohydrate diets and exercise. Nutr Metab (Lond). 2004;1(1):7.

13. Stanley IA, Ribeiro SM, Gimenez-Cassina A, Norberg E, Danial NN. Changing appetites: the adaptive advantages of fuel choice. Trends Cell Biol. 2014;24(2):118-27.

14. Hasselbalch SG, Knudsen GM, Jakobsen J, Hageman LP, Holm S, Paulson OB. Blood-brain barrier permeability of glucose and ketone bodies during short-term starvation in humans. Am J Physiol. 1995;268(6 Pt 1):E1161-6.

15. Scholl-Burgi S, Holler A, Pichler K, Michel M, Haberlandt E, Karall D. Ketogenic diets in patients with inherited metabolic disorders. J Inherit Metab Dis. 2015;38(4):765-73.

16. Bough KJ, Wetherington J, Hassel B, Pare JF, Gawryluk JW, Greene JG, et al. Mitochondrial biogenesis in the anticonvulsant mechanism of the ketogenic diet. Ann Neurol. 2006;60(2):22335.

17. Schwartzkroin PA. Mechanisms underlying the anti-epileptic efficacy of the ketogenic diet. Epilepsy Res. 1999;37(3):171-80.

18. Xu XP, Erichsen D, Borjesson SI, Dahlin M, Amark P, Elinder F. Polyunsaturated fatty acids and cerebrospinal fluid from children on the ketogenic diet open a voltage-gated $\mathrm{K}$ channel: a putative mechanism of antiseizure action. Epilepsy Res. 2008;80(1):57-66.

19. Fraser DD, Whiting S, Andrew RD, Macdonald EA, Musa-Veloso K, Cunnane SC. Elevated polyunsaturated fatty acids in blood serum obtained from children on the ketogenic diet. Neurology. 2003;60(6):1026-9. 
20. Maalouf M, Rho JM, Mattson MP. The neuroprotective properties of calorie restriction, the ketogenic diet, and ketone bodies. Brain Res Rev. 2009;59(2):293-315.

21. Kim DY, Rho JM. The ketogenic diet and epilepsy. Curr Opin Clin Nutr Metab Care. 2008;11(2):11320.

22. Jeong EA, Jeon BT, Shin HJ, Kim N, Lee DH, Kim HJ, et al. Ketogenic diet-induced peroxisome proliferator-activated receptor-gamma activation decreases neuroinflammation in the mouse hippocampus after kainic acid-induced seizures. Exp Neurol. 2011;232(2):195-202.

23. Steriade C, Andrade DM, Faghfoury H, Tarnopolsky MA, Tai P. Mitochondrial encephalopathy with lactic acidosis and stroke-like episodes (MELAS) may respond to adjunctive ketogenic diet. Pediatr Neurol. 2014;50(5):498-502.

24. Paoli A, Bianco A, Damiani E, Bosco G. Ketogenic diet in neuromuscular and neurodegenerative diseases. Biomed Res Int. 2014;2014:474296.

25. Hughes SD, Kanabus M, Anderson G, Hargreaves IP, Rutherford T, O'Donnell M, et al. The ketogenic diet component decanoic acid increases mitochondrial citrate synthase and complex I activity in neuronal cells. J Neurochem. 2014;129(3):426-33.

26. Frey S, Geffroy G, Desquiret-Dumas V, Gueguen N, Bris C, Belal S, et al. The addition of ketone bodies alleviates mitochondrial dysfunction by restoring complex I assembly in a MELAS cellular model. Biochim Biophys Acta. 2017;1863(1):284-91.

27. Sgobbo P, Pacelli C, Grattagliano I, Villani G, Cocco T. Carvedilol inhibits mitochondrial complex I and induces resistance to $\mathrm{H} 2 \mathrm{O} 2$-mediated oxidative insult in $\mathrm{H} 9 \mathrm{C} 2$ myocardial cells. Biochim Biophys Acta. 2007;1767(3):222-32.

28. Nijtmans LG, Henderson NS, Holt IJ. Blue Native electrophoresis to study mitochondrial and other protein complexes. Methods. 2002;26(4):327-34.

29. Rak M, Rustin P. Supernumerary subunits NDUFA3, NDUFA5 and NDUFA12 are required for the formation of the extramembrane arm of human mitochondrial complex I. FEBS letters. 2014;588(9):1832-8.

30. Lazarou M, Thorburn DR, Ryan MT, McKenzie M. Assembly of mitochondrial complex I and defects in disease. Biochim Biophys Acta. 2009;1793(1):78-88.

31. Andrews B, Carroll J, Ding S, Fearnley IM, Walker JE. Assembly factors for the membrane arm of human complex I. Proc Natl Acad Sci U S A. 2013;110(47):18934-9.

32. Mimaki M, Wang X, McKenzie M, Thorburn DR, Ryan MT. Understanding mitochondrial complex I assembly in health and disease. Biochim Biophys Acta. 2012;1817(6):851-62.

33. Alston CL, Compton AG, Formosa LE, Strecker V, Olahova M, Haack TB, et al. Biallelic Mutations in TMEM126B Cause Severe Complex I Deficiency with a Variable Clinical Phenotype. Am J Hum Genet. 2016;99(1):217-27.

34. Sanchez-Caballero L, Ruzzenente B, Bianchi L, Assouline Z, Barcia G, Metodiev MD, et al. Mutations in Complex I Assembly Factor TMEM126B Result in Muscle Weakness and Isolated Complex I Deficiency. Am J Hum Genet. 2016;99(1):208-16.

35. McKenzie M, Ryan MT. Assembly factors of human mitochondrial complex I and their defects in disease. IUBMB life. 2010;62(7):497-502.

36. Rhein VF, Carroll J, Ding S, Fearnley IM, Walker JE. NDUFAF5 Hydroxylates NDUFS7 at an Early Stage in the Assembly of Human Complex I. The Journal of biological chemistry. 2016;291(28):1485160.

37. Maassen JA, Romijn JA, Heine RJ. Fatty acid-induced mitochondrial uncoupling in adipocytes as a key protective factor against insulin resistance and beta cell dysfunction: a new concept in the pathogenesis of obesity-associated type 2 diabetes mellitus. Diabetologia. 2007;50(10):203641. 
38. Abe Y, Sakairi T, Beeson C, Kopp JB. TGF-beta1 stimulates mitochondrial oxidative phosphorylation and generation of reactive oxygen species in cultured mouse podocytes, mediated in part by the mTOR pathway. American journal of physiology Renal physiology. 2013;305(10):F1477-90.

39. Hirabara SM, Silveira LR, Alberici LC, Leandro CV, Lambertucci RH, Polimeno GC, et al. Acute effect of fatty acids on metabolism and mitochondrial coupling in skeletal muscle. Biochim Biophys Acta. 2006;1757(1):57-66.

40. Pfleger J, He M, Abdellatif M. Mitochondrial complex II is a source of the reserve respiratory capacity that is regulated by metabolic sensors and promotes cell survival. Cell death \& disease. 2015;6:e1835.

41. Zhang J, Nuebel E, Wisidagama DR, Setoguchi K, Hong JS, Van Horn CM, et al. Measuring energy metabolism in cultured cells, including human pluripotent stem cells and differentiated cells. Nat Protoc. 2012;7(6):1068-85.

42. Yamamoto H, Morino K, Mengistu L, Ishibashi T, Kiriyama K, Ikami T, et al. Amla Enhances Mitochondrial Spare Respiratory Capacity by Increasing Mitochondrial Biogenesis and Antioxidant Systems in a Murine Skeletal Muscle Cell Line. Oxidative medicine and cellular longevity. 2016;2016:1735841.

43. Samartsev VN, Smirnov AV, Zeldi IP, Markova OV, Mokhova EN, Skulachev VP. Involvement of aspartate/glutamate antiporter in fatty acid-induced uncoupling of liver mitochondria. Biochim Biophys Acta. 1997;1319(2-3):251-7.

44. Golubitzky A, Dan P, Weissman S, Link G, Wikstrom JD, Saada A. Screening for active small molecules in mitochondrial complex I deficient patient's fibroblasts, reveals AICAR as the most beneficial compound. PLoS One. 2011;6(10):e26883.

45. Watt MJ, Hoy AJ, Muoio DM, Coleman RA. Distinct roles of specific fatty acids in cellular processes: implications for interpreting and reporting experiments. American journal of physiology Endocrinology and metabolism. 2012;302(1):E1-3.

46. Kanabus M, Fassone E, Hughes SD, Bilooei SF, Rutherford T, Donnell MO, et al. The pleiotropic effects of decanoic acid treatment on mitochondrial function in fibroblasts from patients with complex I deficient Leigh syndrome. J Inherit Metab Dis. 2016;39(3):415-26.

47. Miglio G, Rosa AC, Rattazzi L, Collino M, Lombardi G, Fantozzi R. PPARgamma stimulation promotes mitochondrial biogenesis and prevents glucose deprivation-induced neuronal cell loss. Neurochemistry international. 2009;55(7):496-504.

48. McAndrew RP, Wang Y, Mohsen AW, He M, Vockley J, Kim JJ. Structural basis for substrate fatty acyl chain specificity: crystal structure of human very-long-chain acyl-CoA dehydrogenase. The Journal of biological chemistry. 2008;283(14):9435-43. 


\section{Supplementary data}

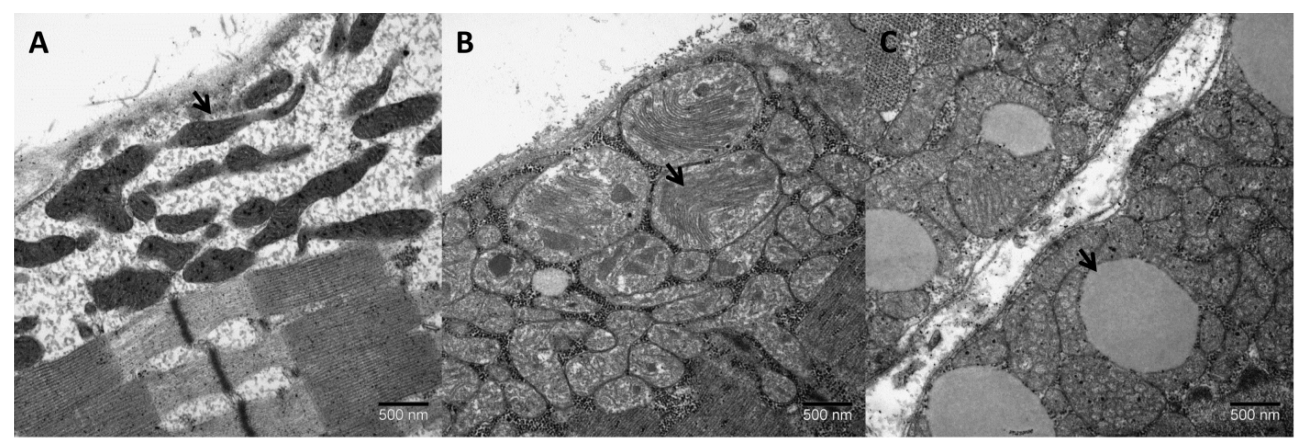

Figure S1: Electron microscopy on patient muscle biopsy (22,000x magnification). (A) elongated and abnormally shaped mitochondria in the subsarcolemmal regions (arrow), (B) enlarged mitochondria and dispersed dense bodies with abnormal cristae and crystalline inclusions (arrow). (C) Large lipid droplets were present between the mitochondria (arrow).

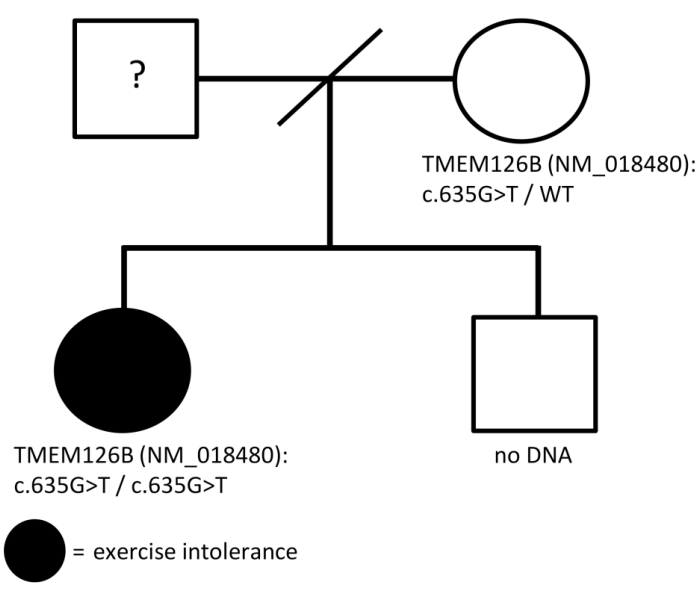

Figure S2: Family of the patient with exercise intolerance and complex I deficiency. All family members, except the patient, were unaffected. Sanger sequencing confirmed that the patient was homozygous for the p.G212V substitution in TMEM126B (NM_018480.4:C.635G>T). Her mother was heterozygous. Other family members were not available for analysis. 


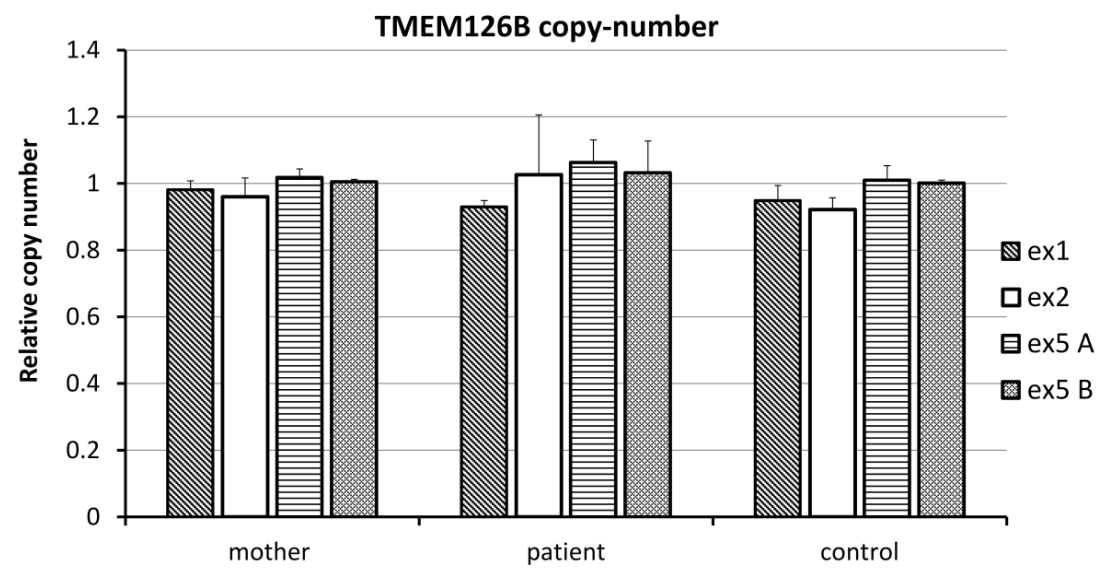

Figure S3: TMEM126B copy-number quantified in the TMEM126B patient, mother of the patient, and control by the 7900HT Fast Real-Time PCR System, normalizing to B2M (beta-2-microglobulin gene, NC_000015.10). Primers were designed in TMEM126B exon 1, 2 and 5 (Supplementary Table S2) and copy numbers were relative to the heterozygous mother. No indications for hemizygosity were found.
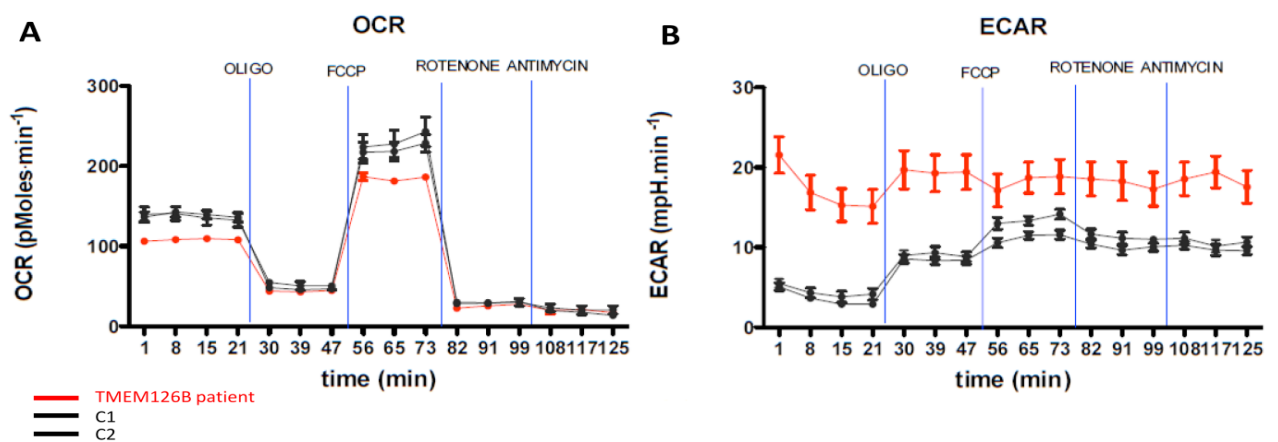

Figure S4: (A) Oxygen consumption rate (pMoles/min) and (B) acidification rates ( $\mathrm{mpH} / \mathrm{min})$ measured in patient fibroblasts (TMEM126B patient) and healty control fibroblasts (C1 and C2) on the Seahorse system. The patient showed normal proton leakage, whereas basal respiration levels, ATP production and maximum respiratory capacity were decreased. Patient fibroblasts were more dependent on glycolytic metabolism than healthy controls. 


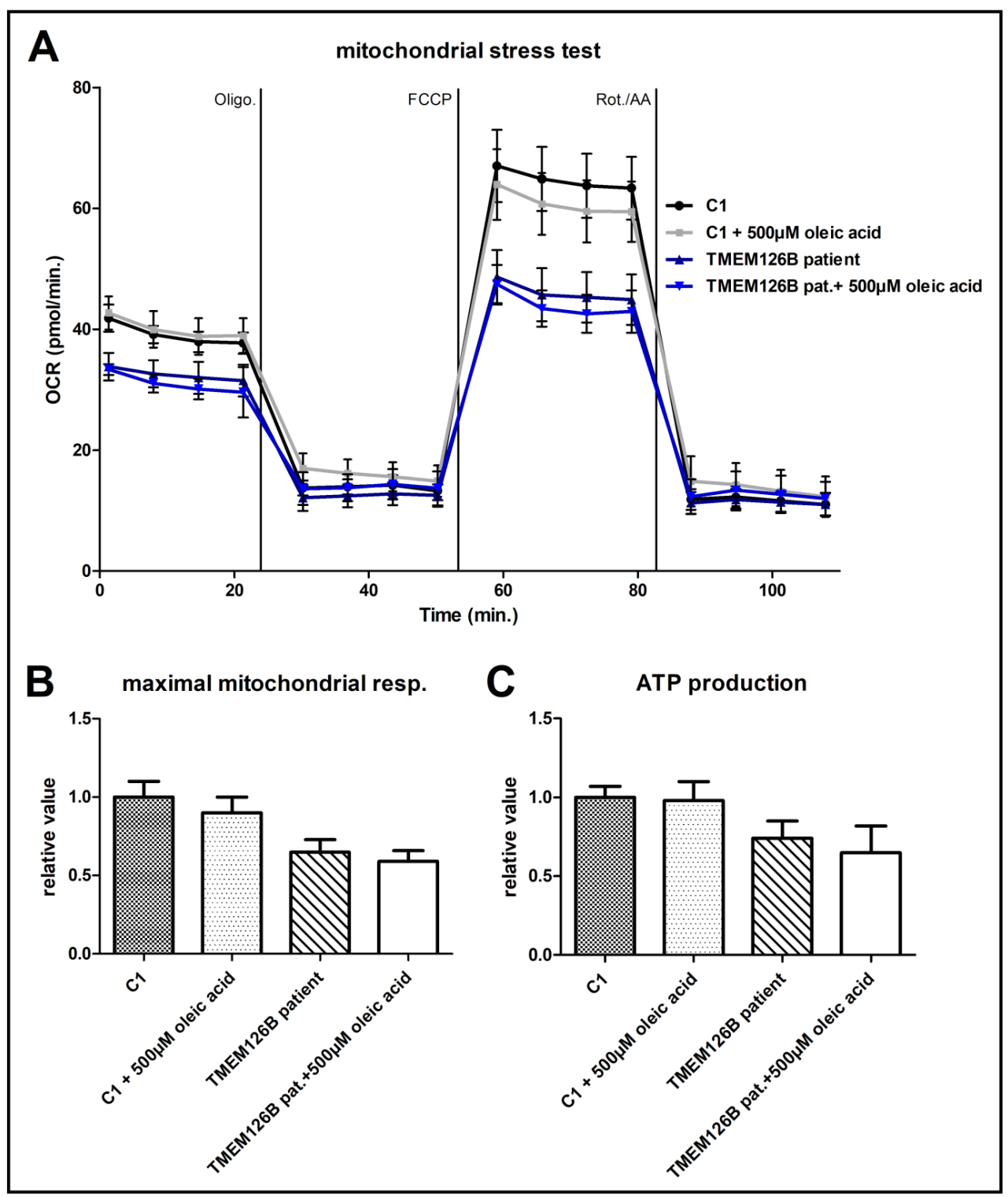

Figure S5: Oxygen consumption rates (pMoles/min) measured in TMEM126B patient fibroblasts and healthy control fibroblasts (C1) after $16 \mathrm{~h}$ treatment with $500 \mu \mathrm{M}$ oleic acid. No significant changes in OCR were observed as a result of oleic acid treatment. 


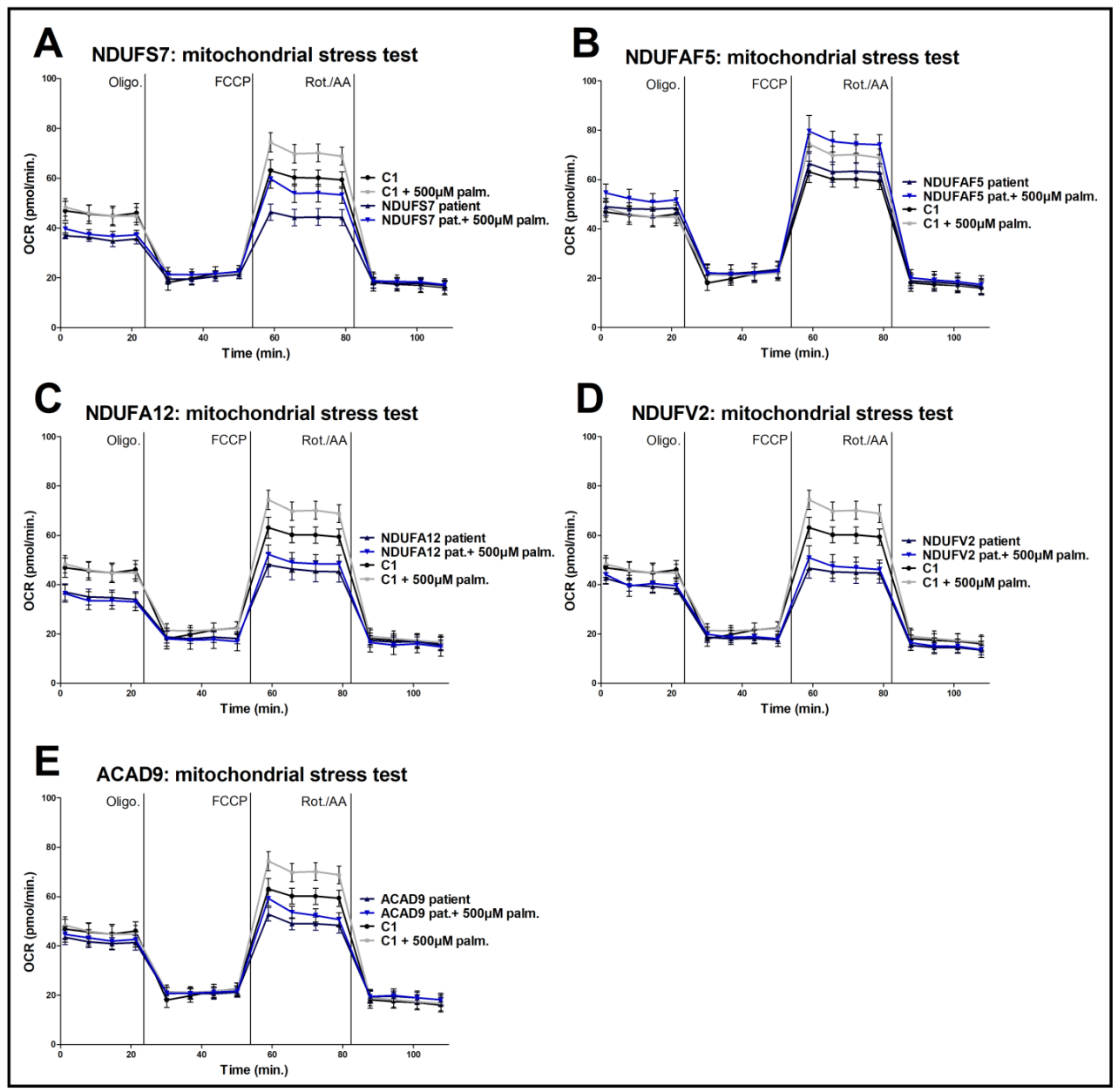

Figure S6: Oxygen consumption rates (pMoles/min) measured in healthy control fibroblasts (C1) and patient fibroblasts with different $\mathrm{Cl}$ defects, all resulting in a $\mathrm{Cl}$ deficiency in muscle. OCR was measured after $16 \mathrm{~h}$ treatment with $500 \mu \mathrm{M}$ palmitate. A significant increase in maximal respiratory capacity was measured in (A) NDUFS7 and (B) NDUFAF5 patient cells after palmitate treatment, whereas (C) NDUFA12, (D) NDUFV2, and (E) ACAD9 defective patient cells were not significantly influenced. 
Table S1: Overview of the dietary study results.

\begin{tabular}{|c|c|c|}
\hline Diet & $\begin{array}{l}\text { Persistence (min.) of bicycle } \\
\text { endurance test } \\
\text { at } 15 \% \text { of } W_{\max }\end{array}$ & $\begin{array}{l}\text { Mean oxygen consumption } \\
\left(\mathrm{VO}_{2 \mathrm{man}}\right) \text { during bicycle endurance } \\
\text { test }(\mathrm{ml} / \mathrm{kg} / \mathrm{min} \text {.) }\end{array}$ \\
\hline $\begin{array}{l}\text { usual diet } \\
\text { (34,5\% of energy from fat) }\end{array}$ & $60 \mathrm{~min}$. & $7,45 \mathrm{ml} / \mathrm{kg} / \mathrm{min}$. \\
\hline $\begin{array}{l}3 \text { weeks high-carbohydrate diet } \\
\text { ( } 25,0 \% \text { of energy from fat) }\end{array}$ & $62 \min$. & $6,82 \mathrm{ml} / \mathrm{kg} / \mathrm{min}$ \\
\hline $\begin{array}{l}3 \text { weeks high-fat diet } \\
\text { ( } 55,0 \% \text { of energy from fat) }\end{array}$ & $\begin{array}{l}95 \mathrm{~min} . \text { ( } 58 \% \uparrow \text { and } 53 \% \uparrow \text { with } \\
\text { respect to usual diet and high } \\
\text { carbohydrate diet, respectively) }\end{array}$ & $\begin{array}{l}8,51 \mathrm{ml} / \mathrm{kg} / \mathrm{min} . \text { ( } 14 \% \uparrow \text { and } \\
25 \% \uparrow \text { with respect to usual } \\
\text { diet and high carbohydrate diet, } \\
\text { respectively) }\end{array}$ \\
\hline \multicolumn{3}{|l|}{ Substrate infusion } \\
\hline $\begin{array}{l}\text { glucose infusion } \\
\text { (10 mg/kg/min.) }\end{array}$ & $65 \mathrm{~min}$. & $5,09 \mathrm{ml} / \mathrm{kg} / \mathrm{min}$ \\
\hline $\begin{array}{l}\text { lipid infusion } \\
\text { (3,7 } \mathrm{mg} / \mathrm{kg} / \mathrm{min} .)\end{array}$ & $\begin{array}{l}90 \mathrm{~min} . \text { ( } 38 \% \uparrow \text { with respect to } \\
\text { glucose infusion) }\end{array}$ & $\begin{array}{l}7,45 \mathrm{ml} / \mathrm{kg} / \mathrm{min} . \text { ( } 46 \% \uparrow \text { with } \\
\text { respect to glucose infusion) } \\
\begin{array}{l}\text { - higher muscle strength } \\
\text { of the lower extremities }\end{array}\end{array}$ \\
\hline
\end{tabular}

Table S2: Primers for Sanger sequencing.

\begin{tabular}{ll}
\hline Sanger primer & $\mathbf{5}^{\prime}$-3' sequence (M13-tailed) \\
\hline FWD (ex5) & TGTAAAACGACGGCCAGTCCACCAAAAGGAAGGGTTTT \\
REV (ex5) & CAGGAAACAGCTATGACCCAGCAATGCCTGAGTCCTCT \\
\hline
\end{tabular}

Table S3: Primers for TMEM126B copy-number determination.

\begin{tabular}{lll}
\hline primer & Fwd primer $\left(\mathbf{5}^{\prime} \mathbf{3}^{\prime} \mathbf{\prime}\right)$ & Rev primer $\left(\mathbf{5}^{\prime}-\mathbf{3}^{\prime}\right)$ \\
\hline exon $\mathbf{1}$ & TAAGCCAAGGGATTCAGGTG & CATGGACTTGGGAGCTGTTT \\
exon $\mathbf{2}$ & CGACACAGTACCTGGCACAC & AGAGAAGGACTGGGCTGACC \\
exon $\mathbf{5}$ A & TGACGCTTTGTCAAACACAA & TCAAGTGTCTCTTCAAATACTGCAT \\
exon $\mathbf{5}$ B & AGCATTCATATCCAGGAGAGGT & CCAAACATAATCTGAAAGACTAGAGG \\
\hline
\end{tabular}




\section{CHAPTER 6}

\section{An ERBB2 mutation as a potential, novel cause of Hirschprung's \\ disease}

Tom E.J. Theunissen*, Debby M.E.I. Hellebrekers*, Bart de Koning, Mike Gerards, Radek Szklarczyk, Elvira N. M. Mulder-Den Hartog, Irenaeus F.M. de Coo, Hubert J.M. Smeets.

*= Authors have contributed equally

Manuscript in preparation 


\begin{abstract}
Hirschsprung disease (HSCR) is characterized by a lack of enteric neurons in the distal part of the colon, resulting in partial or complete bowel obstruction. HSCR develops when neural crest derived cells fail to colonize the distal gastrointestinal tract during early embryogenesis, resulting in an absence of ENS ganglia. Although, several gene defects were shown to cause $\mathrm{HSCR}$, leading to failing migration of the neural crest-derived cells, many HSCR cases remain genetically undiagnosed. Also, little is known about the genes involved in the postnatal maintenance of the ENS. We have performed WES in a HSCR patient with left-sided ptosis from consanguineous parents, and identified a, non-annotated, homozygous c. $2129 \mathrm{C}>\mathrm{T}$ missense mutation in exon 18 of the ERBB2 gene, located in a homozygosity region that overlapped with the patient's affected brother. ERBB2 is significantly expressed in colon epithelial tissue and is an excellent candidate gene, based on the phenotypic similarity between our patient and a previously reported conditional erbB2-KO mutant mouse, in which a lack of functional erbB2 expression affected the postnatal maintenance of the colonic enteric nerves and glial cells, mimicking the human HSCR phenotype. Also, ptosis was present in these mutant mice. Based on this model, we would assume a loss-of-function mutation in our patient. In ERBB2 only few loss-of function (LOF) mutations have been annotated in the SNP-databases, but no homozygous LOF mutation. The mutation identified resulted in a predicted pathogenic p.(Ala710Val) substitution in the Juxta-membrane domain of the ERBB2 receptor, but could also create a donor splice site. When experimentally tested, the c.2129C>T mutation did not lead to alternative splicing or non-sense mediated decay in the patient's fibroblasts. Furthermore, transfection of mutant ERBB2 in HEK293 cells showed no indications that ERBB2 activity was altered due to the c.2129C>T mutation, based on the normal receptor's phosphorylation status and downstream signaling activity. Also, membrane localization was not affected by the ERBB2 mutation. At this stage, we cannot unequivocally demonstrate that the variant causes Hirschsprung disease in our family. Evidence for a definite role for ERBB2 in HSCR awaits further functional validation in an animal model, for which we are currently exploring zebrafish.
\end{abstract}




\section{Introduction}

Hirschsprung disease (HSCR), or congenital a-ganglionic megacolon, occurs in both familial and sporadic form, and is the most prevalent form of congenital bowel obstruction (1/50009000 births) $(1,2)$. HSCR is characterized by a lack of enteric neurons in the myenteric and submucosal plexuses of the distal colon, most often affecting the rectosigmoid region, resulting in partial or complete bowel obstruction. Consequentially, patients suffer from poor feeding and weight gain with sometimes even enterocolitis or colonic rupture involved, if the patient is not diagnosed and treated by colon resection at an early stage (3). The enteric nervous system (ENS) is a complex network of interconnected neurons, which is formed through migration and differentiation of neural crest-derived progenitor cells, developing the sensory neurons, motor neurons, interneurons, and enteric glia that are crucial for the functioning of the gastrointestinal tract. The neurons and glial cells of the ENS are grouped into ganglia which form the myenteric (Auerbach) plexus, located between the longitudinal and circular muscle layers of the gut wall, and submucosal (Meissner) plexus, which lies within the submucosal layer between the external musculature and the mucosa of the small and large intestines. When cells derived from the neural crest (NC) fail to colonize the distal gastrointestinal tract during early embryogenesis this can result in an absence of the ENS ganglia in the colon, causing HSCR (2-5). Mice models have improved our understanding of genes, involved in embryonic development and differentiation of the ENS. Mutant mice (among others: GDNF, NRTN, c-ret, GFR $\alpha 1, G F R \alpha 2, N T-3, \operatorname{trkC}, E D N$ 3, ETB, CNTFR $\alpha$, SOX10, PHOX2b) showed a lack of specific subsets of enteric neurons or aganglionosis of the terminal colon (6). Mutations in RET, EDN-3, ETB, GDNF and SOX10 were most often involved in patients with HSCR, yet many HSCR cases remain genetically unexplained. Although, there is quite some knowledge on genes involved during embryonic development of the ENS, genes involved in maintaining the post-natal integrity of the ENS are largely unknown $(3,7,8)$.

We have performed WES on a patient with HSCR and left-sided ptosis, and identified a likely pathogenic missense mutation in the ERBB2 gene, although further studies failed to reveal the functional consequence of the variant. As not all functional studies have yet been performed, a pathological role still remains possible. This is also based on the striking phenotypic similarity with a previously reported conditional erbB2 mutant mouse, in which a lack of functional erbB2 expression affected the postnatal maintenance of the colonic enteric nerves and glial cells, causing enlarged abdomen, a constricted distal colon and a distended proximal colon, mimicking the human HSCR phenotype. Also, ptosis was exhibited by the mutant mice. Our results indicate that genetic defects in the ERBB2 gene might be a cause of HSCR in human disease, although definite proof awaits testing of the variant in a whole organism, like zebrafish. 


\section{Material and Methods}

\section{Patient}

A female patient from consanguineous parents ( $3^{\text {th }}$ degree) had symptoms of HSCR from birth on (characterized by severe intestinal obstruction), in combination with one-sided ptosis. Additionally, signs of axonal polyneuropathy were present. No metabolic abnormalities were identified. Microscopy analysis on colon biopsies from the proximal and distal part of the colon confirmed the diagnosis HSCR as the distal biopsy showed the characteristic lack of ganglion cells at the anal resection part combined with a hyperplasia of nerve fibers in the submucosa. More to the proximal side of the biopsy, ganglion cells were still observed but were reduced in quantity compared to healthy tissue, also here with hyperplasia of the nerve fibers. The proximal colon biopsy showed a normal composition of the colon wall with ganglion cells in both Meissners's plexus and Auerbach's plexus. The affected younger brother had similar manifestations.

\section{Homozygosity mapping and Whole Exome Sequencing (WES)}

Homozygosity mapping and CNV analysis was performed by HumanMapping 250K array (Affymetrix, Santa Clara, California) and Genotyping console 4.0 (Affymetrix). Homozygosity regions were defined by the "Homozygosity" mapper, with a cutoff of $5 \mathrm{MB}$. Exons and flanking introns were captured with SureSelect version 5 exome enrichment kit (Agilent Technologies, Santa Clara, California), including untranslated regions. Sequencing was performed on a HiSeq2000 platform (Illumina, San Diego, California), using a $2 \times 100 \mathrm{bp}$ paired-end setting. Bcl2fastq 1.8.4 (Illumina) allowed basecalling and demultiplexing, and Burrows-Wheeler Aligner 0.5.9 (Broad Institute, Cambridge, Massachusetts) was used for read alignment against human reference genome hg19. Duplicate reads were removed by Picard software suite 1.77 (Broad Institute, Cambridge, Massachusetts) and variant calling was performed with GATK 2.1-8 (Broad Institute). Exome data was filtered for recessive homozygous mutations located within the homozygosity regions. Variants with allele frequencies <1\% (dbSNP137, Exome Aggregation Consortium) were evaluated, covering missense mutations, indels, nonsense mutations, and splice variants. Non-annotated variants were maintained, unless allele frequencies exceeded $5 \%$ prevalence in our in-house patient database. Pathogenicity of nonsynonymous missense mutation was estimated by Polymorphism Phenotyping-2 (PolyPhen-2; Harvard, Boston, Massachusetts) and Protein Variation Effect Analyzer (PROVEAN; J. Craig Venter Institute). Nonsense, frameshift, and splice-site variations were maintained, where in silico predictions on splice variants were performed using Alamut (included Human Splice Finder, NN Splice and Gene Splicer). 


\section{RNA studies}

RNA was isolated from fibroblasts using $1 \mathrm{ml}$ of TRIzol Reagent per $1^{*} 10^{6}$ cells (Thermo Fisher Scientific, Waltham, MA, USA), according to the manufacturer's protocol. 1000ng of RNA was used as input for cDNA synthesis using the qScript cDNA Synthesis Kit (Quanta bio, Beverly, MA, USA). PCR primers were designed in exon 15-17 and flanking exon 18, as indicated in Table 1, and PCR products were visualised on a $2 \%$ agarose gel.

Quantitative real-time PCR was performed on 40x diluted cDNA using a primer-pair in exon 3-4 of the ERBB2 gene, normalized to TBP expression (Table 1). Sensimix Sybr Hi-Rox (Bioline, Taunton, MA, USA) was used for CDNA amplification, and a relative ERBB2 mRNA expression was calculated using the $2^{-\Delta \Delta C t}$ method.

Table 1: Primer sequences for PCR and QPCR.

\begin{tabular}{|c|c|c|}
\hline \multicolumn{2}{|c|}{ ERBB2 PCR primers (NM_004448.3) } & \multirow{2}{*}{$\frac{\text { product length }}{245 \mathrm{bp}}$} \\
\hline ex15 Fwd: 5'-GCCTGTGCCCACTATAAGGA-3' & ex17 Rev: 5'-AAGACCACGACCAGCAGAAT-3' & \\
\hline ex17 Fwd: 5'-CTTTGGGATCCTCATCAAGC-3' & ex20 Rev: 5'-AGGGCATAAGCTGTGTCACC-3' & 398bp \\
\hline ex16 Fwd: 5'-CTGTGTGGACCTGGATGACA-3' & ex21 Rev: 5'-GGACTCTTGACCAGCACGTT-3' & $669 \mathrm{bp}$ \\
\hline \multicolumn{2}{|c|}{ QPCR primer (NM_004448.3) } & product length \\
\hline $\begin{array}{l}\text { ERBB2 } \\
\text { ex3 Fwd: 5'-CCCAGCTCTTTGAGGACAAC-3' }\end{array}$ & $\begin{array}{l}\text { ERBB2 } \\
\text { ex4 Rev: 5'-GGATCAAGACCCCTCCTTTC-3' }\end{array}$ & $153 \mathrm{bp}$ \\
\hline $\begin{array}{l}\text { TBP } \\
\text { ex5 Fwd: 5'-CACGAACCACGGCACTGATT-3' }\end{array}$ & $\begin{array}{l}\text { TBP } \\
\text { ex6 Rev: 5'-TTTTCTTGCTGCCAGTCTGGAC-3' }\end{array}$ & $89 \mathrm{bp}$ \\
\hline
\end{tabular}

\section{Transfection studies}

A pcDNA3 mammalian expression vector containing the ERBB2 cDNA (4400bp insert; NM_001005862) was obtained from Addgene (Mien-Chie Hung Lab) and was used to create an ERBB2 c.2129C>T mutant transcript by side directed mutagenesis using the QuikChange II Site-Directed Mutagenesis Kit (Agilent Technologies, Santa Clara, California, USA) according to the manufacturer's protocol. HEK293 cells were transfected with WT or mutant ERBB2 vector using FuGENE HD transfection reagent in a mass ratio of 3:1 (ERBB2 vector : FuGENE), and incubating the $60 \%$ confluent HEK293 cells for $48 \mathrm{~h}$ at $37 \mathrm{C}^{\circ}$. Quantitative real-time PCR was performed to determine transfection efficiency in the WT and mutant ERBB2 transfected cells using the ERBB2 exon 3-4 primer-pair (which is exon 7-8 in the larger NM_001005862 transcript, yet encodes the similar 1225 amino-acid protein as NM_004448.3), normalized to TBP expression (Table 1). 


\section{Western blot and Immunohistochemistry}

WT and mutant ERBB2 transfected HEK293 cells were incubated with 100ng/ $\mu$ I EGF for $15 \mathrm{~min}$ prior to protein isolation. An NP-40 buffer was used for protein isolation to maintain the phosphorylated status of the ERBB2 receptor, consisting of $50 \mathrm{mM}$ Tris, $150 \mathrm{mM} \mathrm{NaCl}$, $0.1 \%$ SDS, $0.5 \%$ Sodium Deoxycholate, $1 \%$ Igepal CA-630, pH 7.4. Additionally, protease inhibitors (Complete 25x) and phosphatase inhibitors were added (PhosSTOP 20x). Protein concentrations were measured using the Bradford protein assay and $30 \mu \mathrm{g}$ of total protein was loaded to each well of a precast Biorad 4-20\% gradient gel (Mini-PROTEAN TGX Precast Protein Gel). Electrophoresis and blotting were performed using the Biorad Mini-PROTEAN system. The 200kDa ERBB2 and 1221/1222 P-ERBB2 proteins were immunostained with monoclonal Rabbit antibodies (respectively, Abcam: ab214275 and Abcam: ab47263). The $60 \mathrm{kDa}$ Akt and p-Akt proteins were immunostained with monoclonal Rabbit antibody against respectively pan-AKT (Abcam: ab18785) and phospho T308 pan-AKT (Abcam: ab38449). To define the mean P-ERBB2:ERBB2 and P-AKT:AKT ratio's, a relative quantification of the protein bands was performed by Fiji based on the band intensity and selected area size, corrected for background signal.

To compare receptor membrane localization in WT-ERBB2 and mutant-ERBB2 transfected HEK293 cells, immunohistochemistry was performed. Transfected HEK293 cells were seeded at $30 \%$ confluency on a glass coverslip. Fixation was performed by incubating the cells in $4 \%$ paraformaldehyde in PBS pH 7.4 for 10 min. Samples were then incubated in 0.5\% Tween-20 for permeabilization and after blocking in 1\% BSA for 30 min., cells were incubated overnight in 1:4000 primary anti-ERBB2 in 1\% BSA (Abcam: ab214275). Secondary goat anti-rabbit FITC antibody was administered for $1 \mathrm{~h}$ and confocal imaging was performed.

\section{Results}

\section{ERBB2 variant detected by WES in Hirschprung patient}

Based on the presence of 2 mutations in a single gene, and the filtering criteria for pathogenicity and variant allele frequency, a single candidate gene defect was identified by WES. A homozygous c.2129C>T missense mutation in exon 18 of the ERBB2 gene caused a predicted pathogenic $p$.(Ala710Val) substitution that had not been annotated in the SNP databases (dbSNP, ExAC). The variant was located in a homozygosity region, which was shared with her affected brother (50Mb overlap). Both parents were heterozygous. The mutated amino acid position was highly conserved in 100 vertebrates (UCSC, Figure S1), whereas 
a valine residue at p.710 does not naturally exist in other species. In silico pathogenicity prediction tools PROVEAN and PolyPhen 2 classified the mutation as "deleterious" (-2.6 score) and "probably damaging" (0.99 score), respectively. According to NextProt (NX_ P04626) the mutated p.710 amino-acid is located between the transmembrane region (p.653-p.675) of the receptor and the intracellular tyrosine kinase domain (p.720-p.987), both being part of the cytoplasmic domain of the receptor (p.676-p.1255). p.680-p.710 has previously been specified as a Juxta-membrane domain, consisting of a Juxta-membrane subdomain $A(J M-A)$ and subdomain B (JM-B); although the precise function of this domain is currently unclear, the region is evolutionary highly conserved (Figure S2).

According to the gnomAD dataset, a lower than expected number of loss-of function (LOF) mutations (non-sense and frame-shift mutations) have been identified in ERBB2 based on its gene size ( 7 instead of the predicted 50.3) and no homozygous LOF mutations have been reported. In exon 18, which is not a notable smaller exon, only a single heterozygous missense mutation (p.Met712Arg) was identifed in a single patient. The variant in our patient could also affect splicing, as NN Splice (0.7 score), Human Splice Finder (75\% score) and Gene splicer (6.7 score) predicted a potential donor splice site due to c.2129C>T mutation (AG/GT site), which might cause an $81 \mathrm{bp}$ loss of exon 18, corresponding with an in frame deletion of 27 amino acids (Figure 1). Also, potential alternative, natural, cryptic splice sites with high affinity for spliceosome factors were predicted to be present in the surrounding nucleotide sequences (Figure 1). ERBB2 (NM_004448.3) is highly expressed in colon tissue according to Affymetrix micro-array data (NCBI), but similar transcript isoforms, including the mutated exon 18, were expressed in skin fibroblasts (Figure S3), making patient fibroblasts suitable for testing ERBB2 splice variations and mRNA expression levels. 


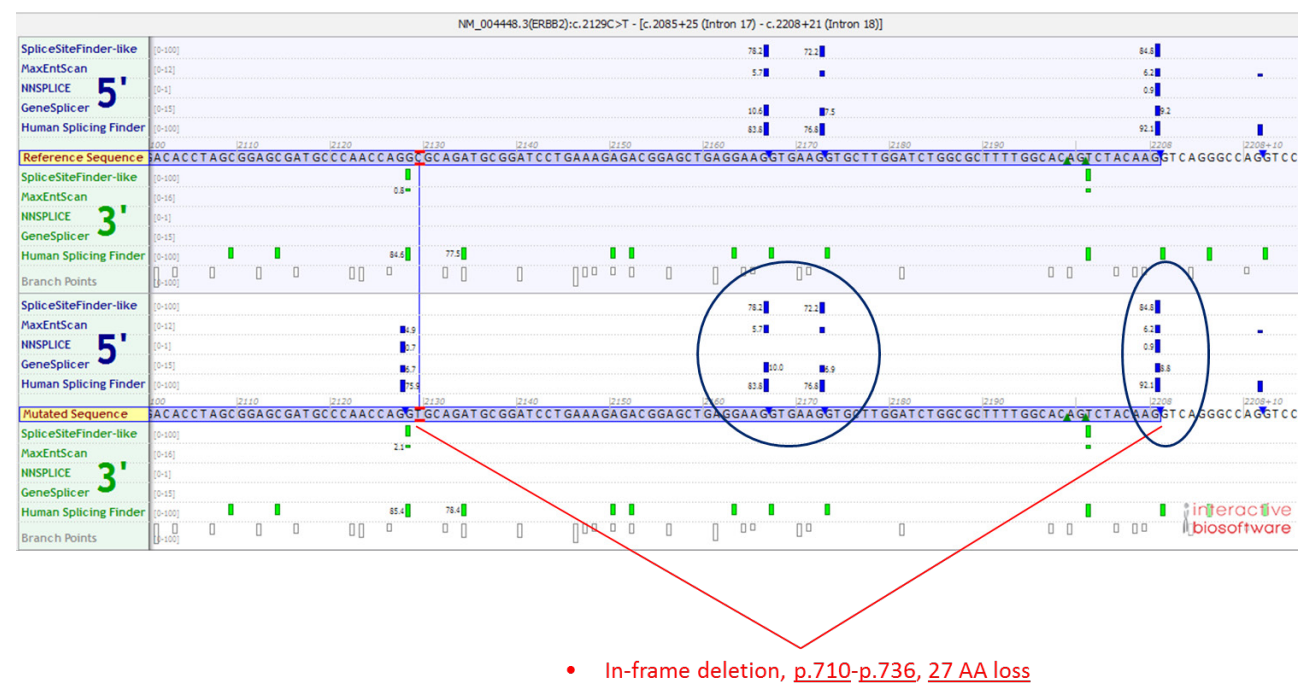

Figure 1: Potential effect of the ERBB2 c.2129C $>$ T variant on splicing. NN Splice (0.7 score), Human Splice Finder (75\%) and Gene splicer (6.7 score) predicted a new potential cryptic donor splice site due to c.2129C $>$ T mutation, which could cause a 81 bp nucleotide loss, corresponding with a deletion of 27 amino acids (in frame). However, potential natural cryptic splice sites were present in the surrounding nucleotide sequences, predicted to have even higher affinity to spliceosome factors.

\section{Effect ERBB2 variant on mRNA splicing and expression}

Two primer sets surrounding the c.2129C>T variant in exon 18, covering a larger 669bp and smaller 398bp fragment, were used for ERBB2 PCR amplification from patient and control cDNA derived from fibroblasts in order to visualize possible cryptic splice events. As a negative control, a primer pair covering exon 15-17 was used (Figure 2A). Additionally, PCR products were Sanger sequenced to reveal possible small splice event. Our results showed that the $c .2129 \mathrm{C}>\mathrm{T}$ variant in the patient did not cause a cryptic donor splice site in exon 18, which might cause a $81 \mathrm{bp}$ loss, or any other form of alternative mRNA splicing, as both exon 18 flanking fragments were the same length (398bp and 660bp) as in control conditions (Figure 2B). To exclude that we missed it due to nonsense mediated decay of the alternatively spliced mRNA, we performed a quantitative real time PCR analysis, which indicated no significant differences in ERBB2 mRNA expression level between fibroblasts of the heterozygous father and patient (Figure 3). 


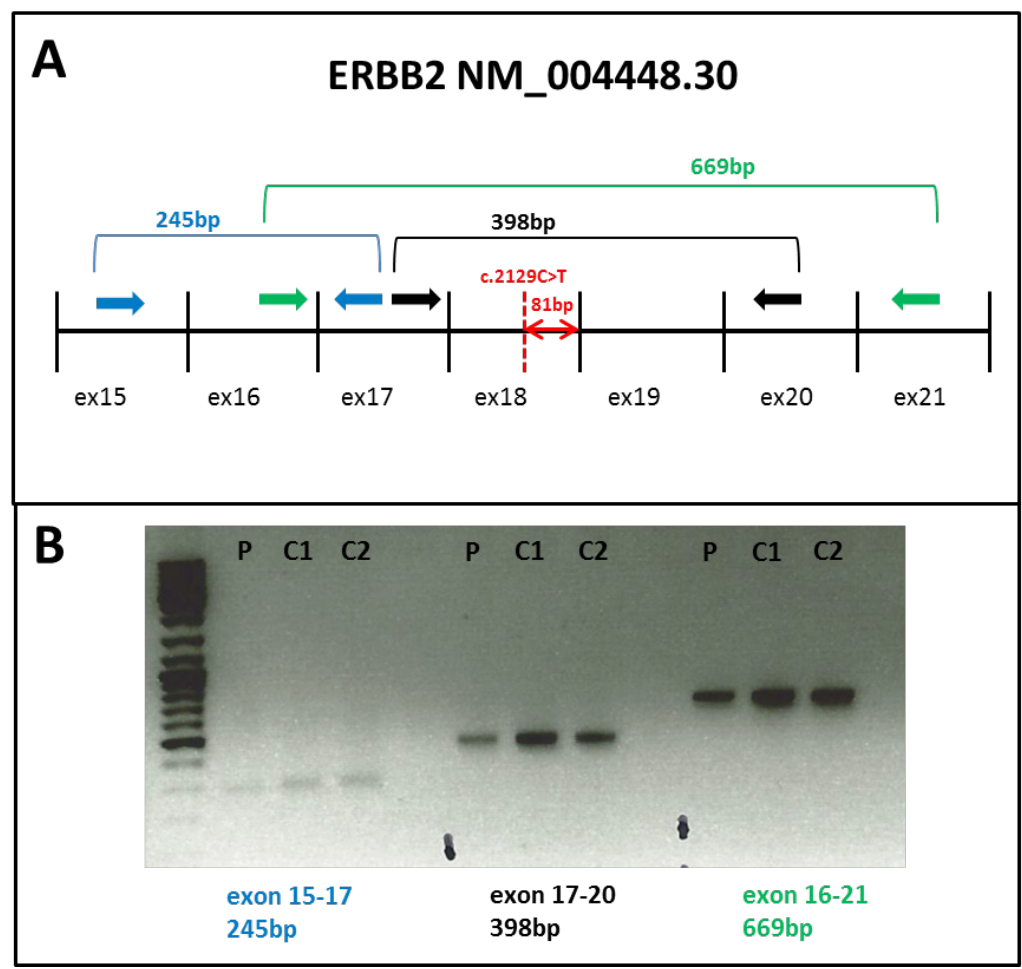

Figure 2: Testing for potential cryptic splice events in the patient's cDNA. (A) Two primer sets surrounding the potential cryptic splice donor site in exon 18, were designed to cover a larger $669 \mathrm{bp}$ and smaller 398bp fragment, and were used for ERBB2 PCR amplification from patient and control cDNA. As a negative control, a primer pair covering 245 bp of exon 15-17 was used. (B) PCR products were visualized on a $2 \%$ agarose gel to reveal possible cryptic splice events in the patient. No indications for cryptic splicing were observed on agarose gel, or after Sanger sequencing of the products.

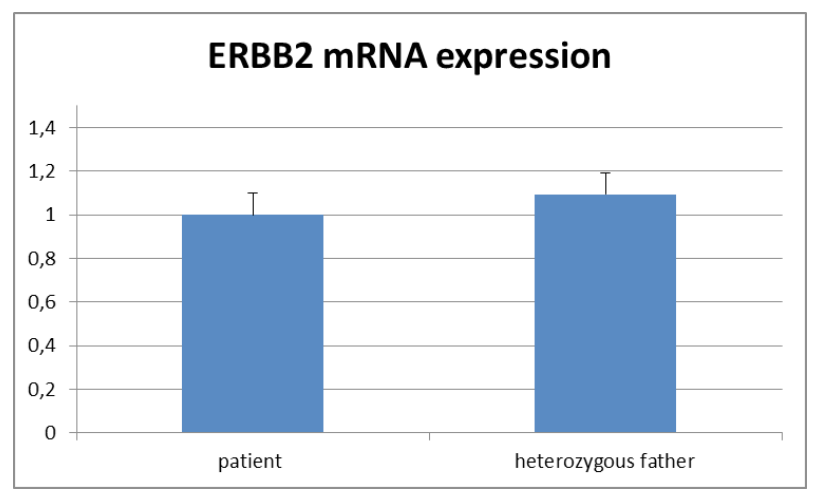

Figure 3: ERBB2 mRNA expression levels in the homozygous Ala710Val patient and heterozygous father. Quantitative real-time PCR was performed to measure ERBB2 expression, based on exon 3-4, in the homozygous Ala710Val patient and heterozygous father. No significant difference was measured in ERBB2 mRNA quantity, indicating non-sense mediated RNA decay is not occurring. 


\section{ERBB2 receptor membrane localization and activity}

The c.2129C>T variant is located just outside an 11 amino-acid sequence (amino-acid 690701, LLQETELVEPLT) of the Juxta-membrane domain of ERBB2, which is necessary for the basolateral membrane localization of ERBB2. Therefore, we tested if there were indications that the mutation affects the localization of ERBB2 to the membrane. As shown in Figure 4, HEK293 cells were transfected with either wild-type (Figure 4A) or mutant ERBB2 (Figure $4 \mathrm{~B})$ and the ERBB2 receptor was stained by immunohistochemistry. Clearly, there was an intense ERBB2 signal present in the outer membranes of almost all wild-type and mutant transfected cells, with no abnormal signs of intracellular accumulation, indicating that the localization of the receptor was not affected by the c.2129C>T mutation.

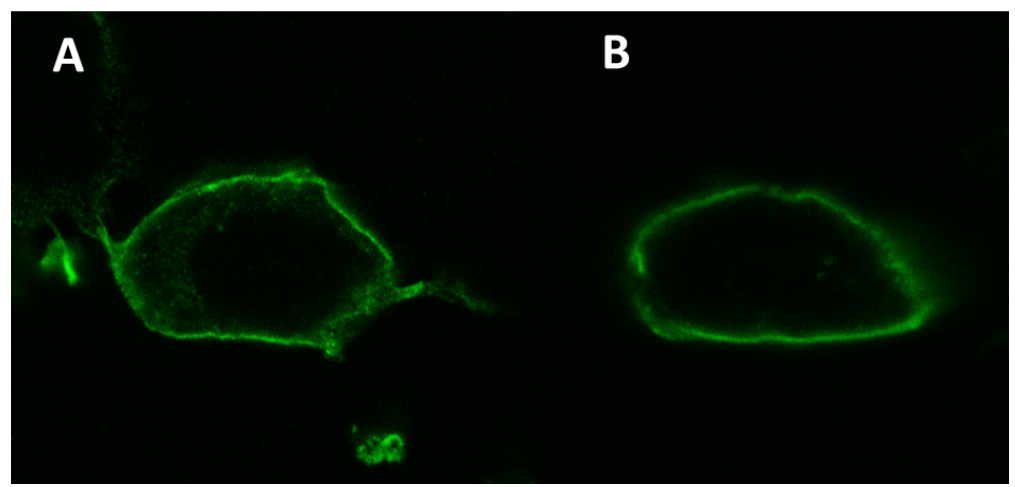

Figure 4: Confocal microscopy after IHC on wild-type (A) and mutant ERBB2 (B) transfected HEK293 cells. A comparable ERBB2 membrane localization pattern was observed in the wild-type and mutant condition, indicating that the c.2129C $>$ T mutation did not severely affect the basolateral sorting signal of ERBB2. Both conditions showed equal intensity in membrane staining, with comparable variation in amount of intracellular ERBB2.

Tyrosine residues 1221/1222 are commonly used to measure ERBB2 activation, as the residues, located at intracellular domain of ERBB2, become phosphorylated upon receptor activation and induce downstream signaling. To test if the c.2129C>T mutation affected the activity of the ERBB2 receptor, we compared the quantitative ratios of total ERBB2 receptor and the phosphorylated ERBB2 receptor (P-ERBB2) at tyrosine residue 1221/1222 (P-ERBB2 Tyr1221/1222) between mutant and wild-type transfected HEK293 cells. We stimulated the ERBB2 activation cascade by pre-treating transfected cells with $100 \mathrm{ng} / \mu$ l epidermal growth factor (EGF) for $15 \mathrm{~min}$, as the activated receptor status (P-ERBB2) is hardly detectable when the EGFR pathway is not stimulated by one of its ligands. Western blot analysis (Figure 5) showed no differences in the P-ERBB2:ERBB2 protein ratios between wild-type and mutant transfected HEK293 cells. Computational quantification of the bands indicated that the difference in P-ERBB2:ERBB2 ratio between both conditions was always less than $10 \%$. 
ERBB2:

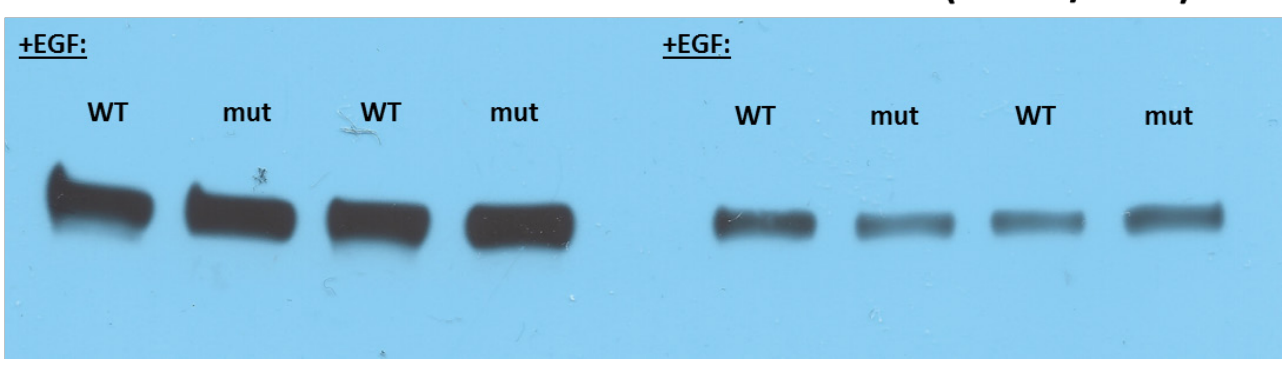

Figure 5: Western blot analysis as a measure for ERRB2 activity. Western blot analysis showed no differences in the P-ERBB2 (Tyr1221/1222):ERBB2 protein ratios between wild-type and mutant transfected HEK293 cells after stimulation of the EGFR pathway with 100ng/ $\mathrm{ll}$ EGF. The difference in P-ERBB2:ERBB2 ratio between both conditions was less than 10\%, indicating that the c.2129C $>T$ mutation did most likely not affect ERBB2 receptor activation.

AKT is an important factor downstream of the ERBB2 signaling cascade, and becomes activated upon phosphorylation at threonine 308 (p.T308), located in the activation loop. The phosphorylation status of P-AKT was shown to increase by both stimulation of the EGFR pathway with ligand and by overexpression of the ERBB2 receptor. We tested if mutant ERBB2 overexpressing HEK293 cells showed altered activation of AKT compared to the wildtype transfected cells by determining the P-AKT (p.T308):AKT ratios after stimulation of the EGFR pathway by $100 \mathrm{ng} / \mu \mathrm{l}$ for $15 \mathrm{~min}$. As shown in Figure 6, both wild-type and mutant transfected HEK293 cells showed strong activation of AKT, where the P-AKT:AKT ratios did not differ more than $5 \%$ between wild-type and mutant condition, indicating that c. $2129 \mathrm{C}>\mathrm{T}$ does not affect the signaling cascade downstream of ERBB2.

AKT:

P-AKT:

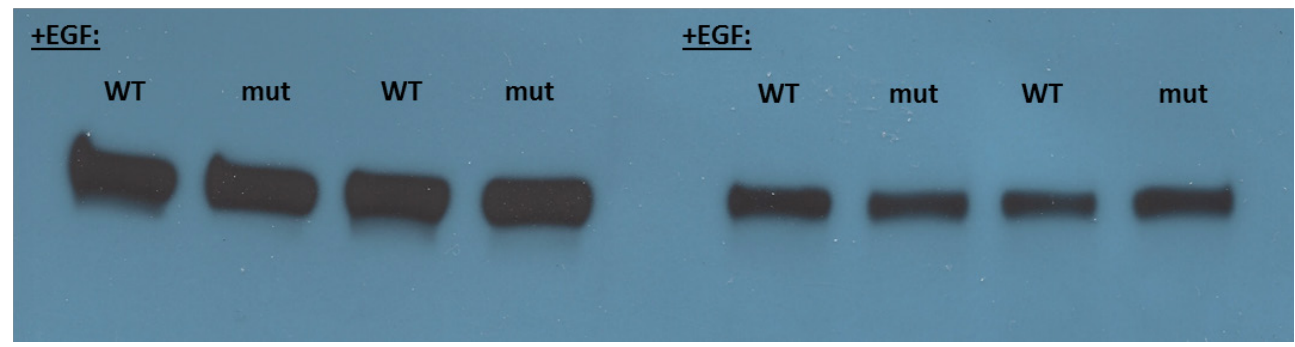

Figure 6: Western blot analysis as a measure for the activity of AKT, functioning downstream of ERBB2. Wild-type and mutant transfected HEK293 cells showed activation of AKT after stimulation with EGF, where the P-AKT:AKT ratios did not differ more than 5\% between wild-type and mutant condition, indicating that c.2129C $>T$ did not drastically affect the signaling cascade downstream of ERBB2. 


\section{Discussion}

WES of a consanguineous patient with HSCR and one-sided ptosis revealed a homozygous c.2129C $>$ T missense mutation in exon 18 of the ERBB2 gene. ERBB2 is significantly expressed in colon tissue and was reported in relation to HSCR in erbB2/nestin-Cre conditional mouse mutants (6). The mutation was predicted to cause a p.(Ala710Val) substitution at a conserved amino-acid position with a damaging effect at protein level. As also a possible effect on splicing was predicted, we tested ERBB2 mRNA in patient fibroblasts and excluded alternative splicing or nonsense mediated decay.

ERBB2 (Her2) belongs to the family of epidermal growth factor receptors (EGFRs), which function as tyrosine kinases in the outer cell membrane. Early evidence suggested that neuregulins (Nrg-1/NRG-1) and their binding receptors, including the ErbB2 receptor heterodimers, have a crucial role in the development of the gastrointestinal tract. ErbB2 and ErbB3 receptors were shown to be expressed in the mucosal epithelia of the colon and small intestine $(9,10)$ and nrg-1 ligand was expressed in the mucosal epithelia and enteric ganglia (11). The crucial function of Nrg-1 and its ErbB2/ErbB3 receptor complex in maintaining the ENS was shown by Nrg1, ErbB3 and ErbB2 K.O. mice. ErbB3-/- mice exhibited severe neuropathies with a complete loss of glia (12) and a decrease in the number of ganglia in the duodenal gut (13). As ErbB2 null mutants died at embryonic day 10.5 due to a lethal heart defect, conditional ErbB2/Nestin-Cre mutant mice were developed to investigate the role for ErbB2 in the postnatal maintanace of the ENS. In these mice expression of ErbB2 was specifically lacking in the ENS and colonic epithelial cells, which resulted in retarded growth, distended colons and premature death. Interestingly, the phenotype was shown to mimic human HSCR and the observed loss of enteric nerves and glia cells in the colon after three weeks is a major criteria for the diagnosis HSCR (6). As the loss of nerves and glia cells occurred postnatally due to a lack of ErbB2 signaling in the colon epithelial cells, it was suggested that ErbB2 is required for post-natal maintanance of the ENS. Further investigations into ERBB2 were triggered by the striking phenotypic similarities between our patient and the erbB2/nestin-Cre conditional mouse mutants. Not only did the mutant mice and our patient both displayed symptoms of HSCR, characterized by a marked loss of enteric glial cells, but also ptosis was present in both. To investigate if the p.(Ala710Val) substitution could explain the symptoms in our patient, we studied ERBB2 function in HEK293 cells overexpressing either wild-type or mutant ERBB2. As a possible role for the juxta-membrane domain in the process of localizing ERBB2 to the basolateral membrane was proposed, we studied ERBB localization by immunohistochemistry. However, we found no indications that the ERBB2 membrane localization was affected by the mutation. This could be due to the fact that p.710 is located just outside an amino-acid domain (p.690- 
701) with an important role as a sorting signal (14). In EGFR receptor 1 (ERBB1), it was reported that the juxta-membrane (JM) segment of EGFR participates in protein kinase activation, because mutations in the C-terminal (residues 664-682) of this region abolished EGFR activation (15). It was concluded that the JM segment of the receiver kinase cradles or surrounds the C-lobe of the donor and that the entire JM segment is required for full activation of the receptor (16-18). Nevertheless, our data showed that the p.(Ala710Val) mutation in the juxta-membrane domain of ERBB2 did not decrease the activation status of the ERBB2 receptor upon stimulation with EGF, nor did we observe any decreased activity in downstream signaling via AKT.

The signaling cascade of the ERBB2 membrane receptor is dependent on heterodimerisation with other members of the EGFR family, including ERBB1, ERBB3, ERBB4, which occurs when receptor ligands such as epidermal growth factor (EGF), Neuregulin 1 or 2 (NRG1, NRG2) or Tumor growth factor alpha (TGF $\alpha$ ) bind to one of the partner receptors (especially ERBB3). However, no high affinity ligand has yet been identified which directly binds to ERBB2, and therefore it has been suggested to act as a co-receptor. Upon dimerization, ERBB2 receptors auto-phosphorylate the tyrosine residues on their intracellular tail section, leading to the activation of the receptor and initiation of a downstream signaling cascade. Shc is an important protein that directly interacts with ERBB2 upon activation by binding its PTB domain to Tyr1196 and Tyr1248, and via its SH2 domain to Tyr1248 and Tyr1221/1222. Although, there is significant overlap in the downstream signaling cascades activated by the combinations of ERBB hetero- or homodimers, signaling via the PI3K-PAKT and MAPK pathway are known to be important regulating factors downstream of ERBB2, stimulating cell proliferation and survival $(17,19)$. In contrast to inactivating mutations, activating mutations in both the extracellular and intracellular domain of ERBB2 have been extensively reported in human cancers as a cause of breast cancer, lung cancer, glioblastoma, ovarian cancer and gastric cancer (20-25), either in combination with ERBB2 amplification or in ERBB2 amplification negative cancers. ERBB2 mutation hotspots are in the intracellular kinase domain and extracellular receptor domain, but mutations were also found in the trans-membrane domain and intracellular tail section (26-29). An p.(Arg678GIn) variant is located at the border of the Juxta-membrane domain in an undefined region, and has been detected in cancer patients (included in the COSMIC database). However, functionally, the mutation did not have a gain or loss of function effect on ERBB2 activation (phosphorylation sites assessed), and should be considered as a variant of unknown significance $(30,31)$. So far, no activating nor inactivating mutations have been reported in the Juxta-membrane domain (p.680-710) of ERBB2, in which we identified the mutation in our patient. According to the gnomAD dataset, a lower than expected number of loss-of function (LOF) mutations (non-sense and frame-shift mutations) have been identified in ERBB2 based on its gene size 
(7 instead of the predicted 50.3) and no homozygous LOF mutation. In exon 18, which is not a notable smaller exon, only a single heterozygous missense mutation (p.Met712Arg) was reported in a single cancer patient. The absence of pathogenic variants supports our hypothesis that this genetic region does not tolerate damaging mutations on both alleles, indicating that the predicted damaging p.(Ala710Val) mutation affects a functionally important and conserved domain of ERBB2.

In conclusion, a likely pathogenic variant in the ERBB2 gene has been identified in a HSCR patient. Inactivating mutations in this gene explained a mouse model for HSCR that showed, similar as our patient, signs of ptosis. Although we have shown that the variant does not lead to activation of ERBB2, we could not demonstrate that the variant inactivates ERBB2. So, evidence for a definite role for ERBB2 in HSCR awaits further functional validation, especially based on the affected nerve tissue, the ENS, where enteric neuronal cell cultures or CRISPR/Cas9 animal models would be preferred models to reveal the tissue specific pathology caused by ERBB2 defects. 


\section{References}

1. Best KE, Addor MC, Arriola L, Balku E, Barisic I, Bianchi F, et al. Hirschsprung's disease prevalence in Europe: a register based study. Birth defects research Part A, Clinical and molecular teratology. 2014;100(9):695-702.

2. Szylberg L, Marszalek A. Diagnosis of Hirschsprung's disease with particular emphasis on histopathology. A systematic review of current literature. Przeglad gastroenterologiczny. 2014;9(5):264-9.

3. Parisi MA. Hirschsprung Disease Overview. In: Pagon RA, Adam MP, Ardinger HH, Wallace SE, Amemiya A, Bean LJH, et al., editors. GeneReviews(R). Seattle WA: University of Washington, Seattle. GeneReviews is a registered trademark of the University of Washington, Seattle; 1993.

4. Heanue TA, Pachnis V. Enteric nervous system development and Hirschsprung's disease: advances in genetic and stem cell studies. Nature reviews Neuroscience. 2007;8(6):466-79.

5. Iwashita T, Kruger GM, Pardal R, Kiel MJ, Morrison SJ. Hirschsprung disease is linked to defects in neural crest stem cell function. Science (New York, NY). 2003;301(5635):972-6.

6. Crone SA, Negro A, Trumpp A, Giovannini M, Lee KF. Colonic epithelial expression of ErbB2 is required for postnatal maintenance of the enteric nervous system. Neuron. 2003;37(1):29-40.

7. Bondurand N, Natarajan D, Barlow A, Thapar N, Pachnis V. Maintenance of mammalian enteric nervous system progenitors by SOX10 and endothelin 3 signalling. Development (Cambridge, England). 2006;133(10):2075-86.

8. Hofstra RM, Osinga J, Buys CH. Mutations in Hirschsprung disease: when does a mutation contribute to the phenotype. European journal of human genetics : EJHG. 1997;5(4):180-5.

9. Press MF, Cordon-Cardo C, Slamon DJ. Expression of the HER-2/neu proto-oncogene in normal human adult and fetal tissues. Oncogene. 1990;5(7):953-62.

10. Prigent SA, Lemoine NR, Hughes CM, Plowman GD, Selden C, Gullick WJ. Expression of the c-erbB-3 protein in normal human adult and fetal tissues. Oncogene. 1992;7(7):1273-8.

11. Orr-Urtreger A, Trakhtenbrot L, Ben-Levy R, Wen D, Rechavi G, Lonai P, et al. Neural expression and chromosomal mapping of Neu differentiation factor to 8p12-p21. Proc Natl Acad Sci U S A. 1993;90(5):1867-71.

12. Riethmacher D, Sonnenberg-Riethmacher E, Brinkmann V, Yamaai T, Lewin GR, Birchmeier C. Severe neuropathies in mice with targeted mutations in the ErbB3 receptor. Nature. 1997;389(6652):725-30.

13. Erickson SL, O'Shea KS, Ghaboosi N, Loverro L, Frantz G, Bauer M, et al. ErbB3 is required for normal cerebellar and cardiac development: a comparison with ErbB2-and heregulin-deficient mice. Development (Cambridge, England). 1997;124(24):4999-5011.

14. Dillon C, Creer A, Kerr K, Kumin A, Dickson C. Basolateral targeting of ERBB2 is dependent on a novel bipartite juxtamembrane sorting signal but independent of the $\mathrm{C}$-terminal ERBIN-binding domain. Mol Cell Biol. 2002;22(18):6553-63.

15. Red Brewer M, Choi SH, Alvarado D, Moravcevic K, Pozzi A, Lemmon MA, et al. The juxtamembrane region of the EGF receptor functions as an activation domain. Molecular cell. 2009;34(6):64151.

16. Boran AD. The regulatory role of the juxtamembrane region in the activity of the epidermal growth factor receptor. Biochemical Society transactions. 2012;40(1):195-9.

17. Jura N, Endres NF, Engel K, Deindl S, Das R, Lamers MH, et al. Mechanism for activation of the EGF receptor catalytic domain by the juxtamembrane segment. Cell. 2009;137(7):1293-307.

18. Hubbard SR. The juxtamembrane region of EGFR takes center stage. Cell. 2009;137(7):1181-3. 
19. Kirouac DC, Du J, Lahdenranta J, Onsum MD, Nielsen UB, Schoeberl B, et al. HER2+ Cancer Cell Dependence on PI3K vs. MAPK Signaling Axes Is Determined by Expression of EGFR, ERBB3 and CDKN1B. PLoS computational biology. 2016;12(4):e1004827.

20. Stephens $\mathrm{P}$, Hunter $\mathrm{C}$, Bignell G, Edkins S, Davies $\mathrm{H}$, Teague J, et al. Lung cancer: intragenic ERBB2 kinase mutations in tumours. Nature. 2004;431(7008):525-6.

21. Yamamoto H, Higasa K, Sakaguchi M, Shien K, Soh J, Ichimura K, et al. Novel germline mutation in the transmembrane domain of HER2 in familial lung adenocarcinomas. Journal of the National Cancer Institute. 2014;106(1):djt338.

22. Xie D, Shu XO, Deng Z, Wen WQ, Creek KE, Dai Q, et al. Population-based, case-control study of HER2 genetic polymorphism and breast cancer risk. Journal of the National Cancer Institute. 2000;92(5):412-7.

23. Jo UH, Han SG, Seo JH, Park KH, Lee JW, Lee HJ, et al. The genetic polymorphisms of HER-2 and the risk of lung cancer in a Korean population. BMC Cancer. 2008;8:359.

24. Bodian DL, McCutcheon JN, Kothiyal P, Huddleston KC, Iyer RK, Vockley JG, et al. Germline variation in cancer-susceptibility genes in a healthy, ancestrally diverse cohort: implications for individual genome sequencing. PLoS One. 2014;9(4):e94554.

25. Wang $X$, Sun $Y$, Wong J, Conklin DS. PPARgamma maintains ERBB2-positive breast cancer stem cells. Oncogene. 2013;32(49):5512-21.

26. Herter-Sprie GS, Greulich H, Wong KK. Activating Mutations in ERBB2 and Their Impact on Diagnostics and Treatment. Frontiers in oncology. 2013;3:86.

27. Bose R, Kavuri SM, Searleman AC, Shen W, Shen D, Koboldt DC, et al. Activating HER2 mutations in HER2 gene amplification negative breast cancer. Cancer discovery. 2013;3(2):224-37.

28. Lee JW, Soung YH, Seo SH, Kim SY, Park CH, Wang YP, et al. Somatic mutations of ERBB2 kinase domain in gastric, colorectal, and breast carcinomas. Clinical cancer research : an official journal of the American Association for Cancer Research. 2006;12(1):57-61.

29. Ross JS, Wang K, Gay LM, Al-Rohil RN, Nazeer T, Sheehan CE, et al. A high frequency of activating extracellular domain ERBB2 (HER2) mutation in micropapillary urothelial carcinoma. Clinical cancer research : an official journal of the American Association for Cancer Research. 2014;20(1):68-75.

30. de Martino $M$, Zhuang $D$, Klatte $T$, Rieken $M$, Roupret $M$, Xylinas $E$, et al. Impact of ERBB2 mutations on in vitro sensitivity of bladder cancer to lapatinib. Cancer biology \& therapy. 2014;15(9):1239-47.

31. Kavuri SM, Jain N, Galimi F, Cottino F, Leto SM, Migliardi G, et al. HER2 activating mutations are targets for colorectal cancer treatment. Cancer discovery. 2015;5(8):832-41. 


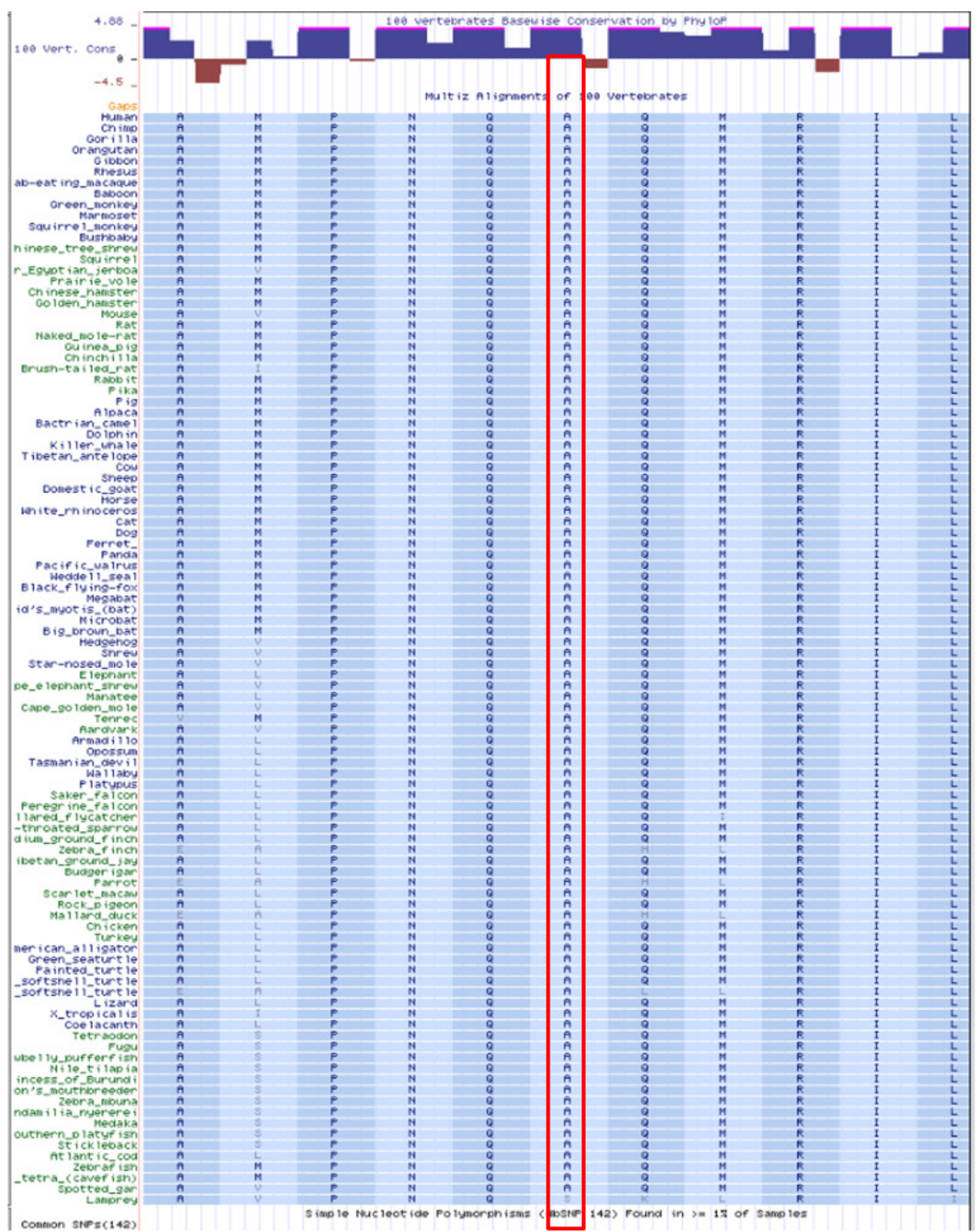

Fig.S1: USCS conservation of ERBB2 amino acid p.710. p.710 is a highly conserved residue in 100 different vertebrates, suggesting that the Ala710Val mutation might affect an important residue. 
Icl|Query_140321

NP 001003817.1

NP 956413.2

Query_140321 1

NP $001003817 \quad 638$

NP $956413 \quad 630$

Query_140321 43

NP $001003817 \quad 718$

NP $956413 \quad 710$
ERBB2 676-719

receptor tyrosine-protein kinase erbB-2 precursor [Mus musculus]

receptor tyrosine-protein kinase erbB-2 precursor [Danio rerio]

Fig.S2: Evolutionary conservation of the ERBB2 juxta-membrane domain (p.680-p.710). Indicating is the amino-acid sequence in human, mice and zebrafish.

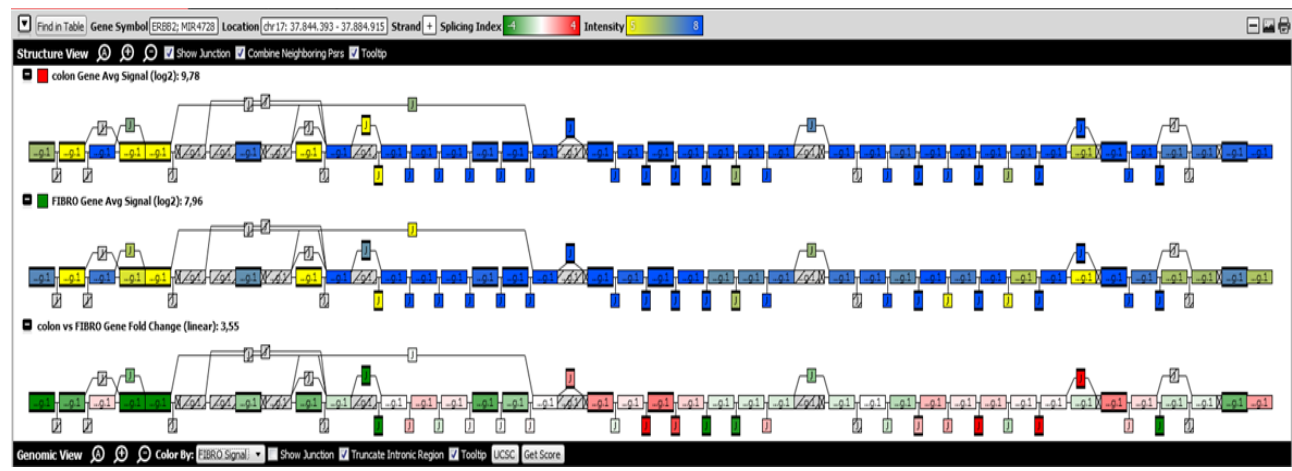

Fig.S3: Affymetrix ERBB2 mRNA expression data (NCBI) from colon tissue (upper) and colon fibroblasts (middle). No large differences in the expression of ERBB2 isoforms (reference: NM_004448.3) are indicated (lower), where exon 18, containing the mutated region was present in both tissue. 


\section{CHAPTER 7}

Differences in strength and timing of the mtDNA bottleneck between zebrafish germline and non-germline cells

Auke B.C. Otten, Tom E.J. Theunissen, Josien G. Derhaag, Ellen H. Lambrichs, Iris B.W. Boesten, Marie Winandy, Aafke P.A. van Montfoort, Katsiaryna Tarbashevich, Erez Raz, Mike Gerards, Jo M. Vanoevelen, Bianca J.C. van den Bosch, Marc Muller, Hubert J.M. Smeets.

Cell reports. 2016;16(3):622-30. doi: 10.1016/j.celrep.2016.06.023 


\begin{abstract}
We studied the mtDNA bottleneck in zebrafish to elucidate size, timing and variation in germline and non-germline cells. Mature zebrafish oocytes contain, on average, $19.0 \times 10^{6}$ mtDNA molecules with high variation between oocytes. During embryogenesis, the mtDNA copy number decreases to 170 mtDNA molecules per primordial germ cell (PGC), a number similar to mammals, and to 50 per non-PGC. These occur at the same developmental stage, implying considerable variation in mtDNA copy number in (non-)PGCs of the same female, dictated by variation in the mature oocyte. The presence of oocytes with low mtDNA numbers, if similar in humans, could explain that (de novo) mutations can reach high mutation loads within a single generation. High mtDNA copy numbers in mature oocytes are established by mtDNA replication during oocyte development. Bottleneck differences between germline and non-germline cells, due to early differentiation of PGCs, may account for different distribution patterns of familial mutations.
\end{abstract}




\section{Introduction}

Various mechanisms have evolved to manage the high mitochondrial DNA (mtDNA) mutation rate (1). In animals, a high mtDNA copy number in cells dilutes the effect of mtDNA mutations (2). Heteroplasmic mutations can only manifest above a tissue- and mutation-specific threshold. Another mechanism is the mtDNA bottleneck during maternal inheritance: a limited amount of mtDNA of the oocytes repopulates the cells of the next generation, thereby filtering out low-level mtDNA mutations. As a result, individuals are usually homoplasmic (3), which is the healthiest situation (4). However, in case of familial pathogenic mutations, the bottleneck can cause high and unpredictable shifts in the mtDNA mutation load and disease risks in the offspring (5).

Despite extensive research, the mtDNA bottleneck is still not fully understood. Data from Holstein cows (6) indicated that the bottleneck was caused by a sharp decrease in mtDNA copy number during early development, most likely, followed by a clonal expansion of these founder molecules during oogenesis. In mice, a similar decrease in the mtDNA copy number between oocytes (range of $\left.10^{5}\right)(7,8)$ and primordial germ cells (PGCs) was reported with 200 mtDNA molecules in a single PGC at the bottom of the bottleneck, in line with previous estimates (185) (9). In contrast, in another study, 2000 mtDNA molecules were measured in a single PGC (10) and a small effective number of segregational units was proposed to explain the rapid segregation, either by assembly of mtDNA molecules into nucleoids or due to preferential replication of a subpopulation of the mtDNA genome $(8,10)$. For salmons, it was reported that the bottleneck occurred during oogenesis with a size of about $85 \mathrm{mtDNA}$ copies (11). In humans, indirect estimations of the bottleneck size have been described, ranging from only 1-5 (12) and 30-35 (13) to 90 (14) and 180 (5) copies.

We measured mtDNA copy number and variation in zebrafish oocytes and in larval germline and non-germline cells during embryonic development. The zebrafish model allowed relatively easy collection of mature oocytes from individual female fish, allowing assessment of the individual variation. Furthermore, PGCs were specifically visualized with GFP followed by fluorescence activated cell sorting (FACS) to isolate both PGCs and nonPGCs during embryogenesis (15). Lastly, oocytes from different stages of development were isolated. In this way, we characterized the mtDNA bottleneck in zebrafish in germline and non-germline cells, providing a better understanding of the underlying mechanism and possible consequences. 


\section{Material and Methods}

\section{Zebrafish embryos and oocytes}

$A B$ zebrafish embryos were raised, housed and staged according to standard procedures (16). Mature and immature oocytes were collected manually as described before (17).

\section{Isolation of primordial germ cells}

Primordial germ cells were identified by both mRNA injections and by a transgenic line. Artificial mRNA constructs with the GFP open reading frame fused to the 3'UTR of zebrafish nanos3 was prepared as described before (15). For the transgenic line, the kop-EGFP-F'nos3-'UTR-cry-DsRed transgene cassette was cloned into the pTolDest vector. Injected or transgenic animals were selected per 100 on the basis of showing similar GFP intensity and no ectopic GFP-expression. Embryos were completely disaggregated and cells were filtered. Subsequently, FACS-sorting (BD Biosciences FACSAria II) was performed as described before (15).

\section{Quantification of mtDNA copy number}

After DNA extraction, the mtDNA copy number was measured. Absolute mtDNA copy number quantification was performed by real-time quantitative PCR (Q-PCR) of the zebrafish mt-nd1 (NADH dehydrogenase 1, mitochondrial) gene. Amplification results were converted to absolute mtDNA copy number using a standard curve. Relative quantification of the mtDNA copy number was performed by Q-PCR measurements of the steady-state amounts of both $m t n d 1$ and $b 2 m$ (nuclear gene beta-2-microglobulin). For the 256-cell stage, we measured the mtDNA copy number both absolutely and relatively, allowing an estimation of the absolute mtDNA copy numbers in embryos staged 512-cell or older (Table S3.1).

\section{Next Generation sequencing}

Next Generation Sequencing (NGS) was performed on an Illumina HiSeq 2000. Heteroplasmy values were calculated as the ration of the nucleotides over the total count of any nucleotide at a position. 


\section{Statistical analysis}

Statistical analyses were carried out using GraphPad Prism Version 5.02 software. Gaussian distribution was tested using the d'Agostino \& Pearson omnibus normality test. One-way analysis of variance (ANOVA) and the Bonferroni multiple comparison test were used for comparing multiple groups. Spearman's rank correlation coefficient was used for analyzing trends. Non-linear regression analysis was performed using the one-phase decay exponential equation. Calculated $P$-values were considered significant $<0.05$. Coefficients of variation (CVs) were calculated as the ratio between the standard deviation and the mean.

\section{Results}

\section{mtDNA copy number and variation in mature zebrafish oocytes}

The average number of mtDNA molecules in 186 individual mature oocytes (Figure 3.1A, S1) was $19.0 \times 10^{6}$ (Range: $3.3 \times 10^{6}-42.2 \times 10^{6}$, Table S3.1), but the distribution was non-Gaussian (Figure 3.1A; P-value: 0.014). We excluded polymorphisms in the primer binding sites as a cause of these variations by sequencing these sites in eight female zebrafish and 10-15 of their oocytes in the $A B$ strain used (Table S3.2(18)). Among these oocytes, 137 were collected from only eight different females [a single batch with ages at both the start (3-6 months) and end of the fertile period (18-24 months)] and the mean values among these females were statistically similar (all $P$-values $>0.05$, Figure $3.1 \mathrm{~B}$ ), with all mean values $\sim 20 \times 10^{6}$, implying no differences in oocytes derived from females from the same batch, early (fish A-D) and late (fish $\mathrm{E}-\mathrm{H}$ ) in their fertile period. The individual frequency distribution of the mtDNA content in oocytes fits a Gaussian distribution ( $P$-values $>0.05)$. High variation in the mtDNA copy number exists within the oocytes from a single female fish (highest value 3-7 times the lowest value, Table S3.1). 
Distribution of mtDNA copy number

A
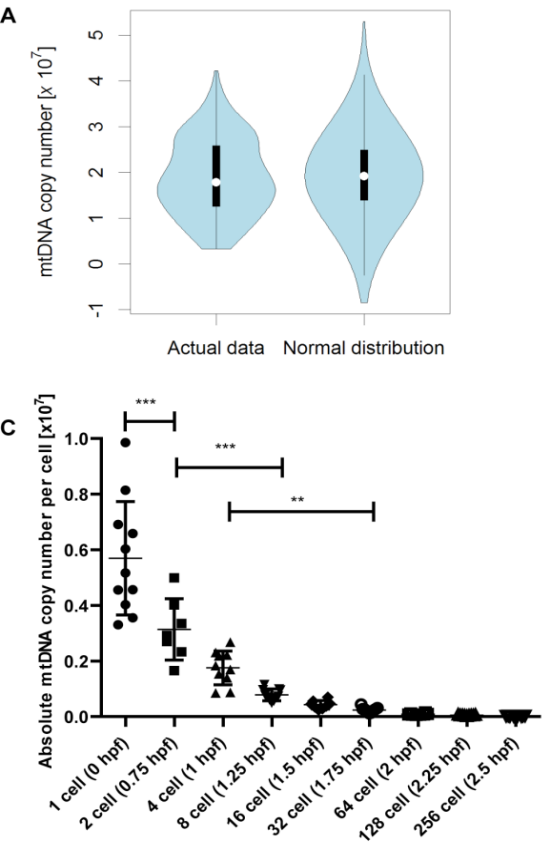

B

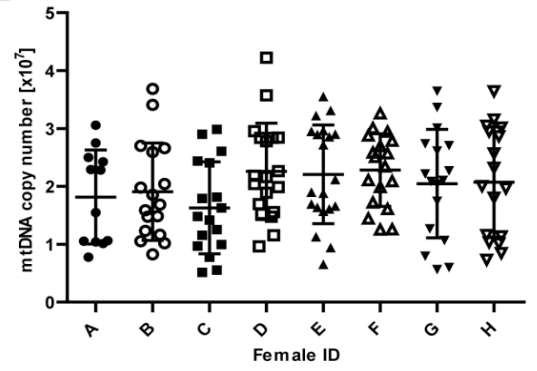

D

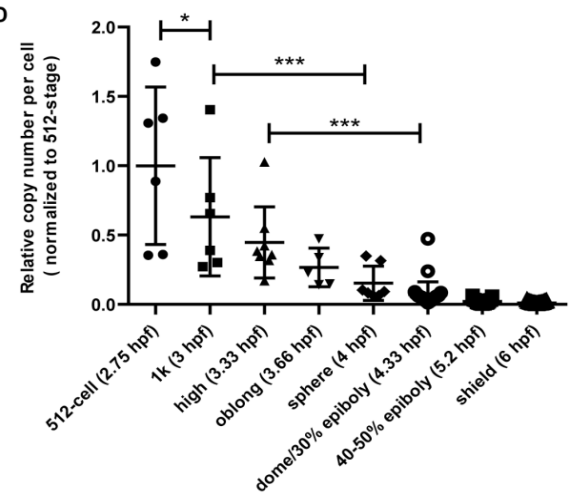

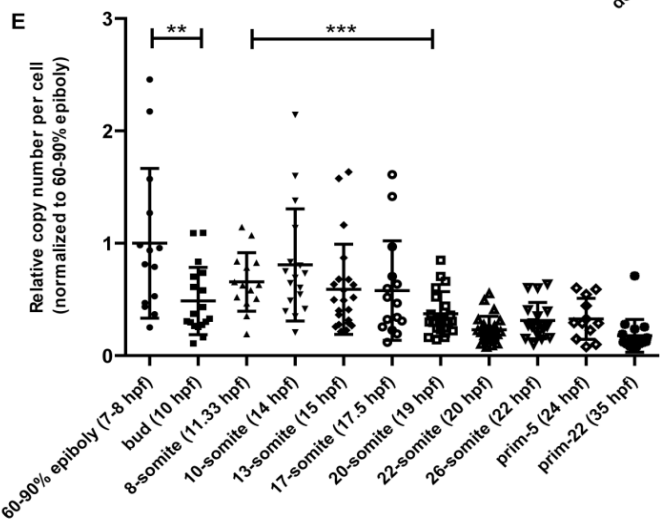

Figure 3.1: The mtDNA copy number in zebrafish mature oocytes and during embryonic development. (A) Violin plot of absolute mtDNA copy number in 186 oocytes (left) compared with that of a Gaussian distribution (with same SD). White dot corresponds to the median value and the black bar to the $25^{\text {th }}$ (bottom) and $75^{\text {th }}$ (top) percentile value. (B) Absolute oocyte mtDNA copy number in the 137 oocytes from eight females, each symbol represents a single oocyte. Fish $A-D$ were at the start and fish $E-H$ at the end of the fertile period. (C) Absolute quantification of the mtDNA copy number per cell in single embryos staged 1-256-cell. (D-E) Relative quantification (mt-nd1/b2m) of the mtDNA copy number (per cell) in single embryos staged (D) 512-cell until shield, normalized to 512-cell stage and (E) 60\%-90 epiboly until prim-22, normalized to the 60-90\% epiboly stage. * P-values $<0.05$, ** P-values $<0.01$, *** P-values $<0.001$ after one-way ANOVA analyis. Horizontal lines indicate the mean value with SD. 


\section{mtDNA copy number during zebrafish embryonic development}

To assess the mtDNA copy number during development we collected embryos and larvae from different stages from one batch of eggs from 5-10 individual females. In the cleavage and early blastula stages, an embryo consists of a fixed number of cells and absolute copy numbers per cell were assessed [1- (mature oocyte) to 256-cell; Figure 3.1C]. The mtDNA copy numbers of the 1-cell stage (mature oocytes) are at the lower bound of the distribution of the mtDNA copy number in all oocytes (Figure 3.1A). As the different isolation methods used gave comparable results (Figure S3.1), this most likely reflects biological differences between the female fish (from different batches). Dividing the mtDNA copy number by the cell number shows that the average mtDNA copy number per cell was halved every cell division, indicating the total number of mtDNA is stable and mtDNA replication is absent.

From the 512-cell stage until the prim-22 stage, we measured the mtDNA copy number relative to a nuclear gene ( $m t n d 1 / b 2 m$, Figure 3.1D-E). The decrease in mtDNA load continued during late blastula -and early gastrula stages (Figure 3.1D), but stopped during segmentation (Figure 1E). Based on the relative and absolute quantification performed on the 256-cell stage, we estimated the amount of mtDNA at this point to be 12-56 mtDNA copies per cell (Table S3.1).

\section{Isolation of germline cells during zebrafish embryogenesis}

PGCs were visualized by injection of egfp-nanos3 3'UTR mRNA and by generating a transgenic line expressing EGFP-nanos3 3'UTR (Figure 3.2A). Both methods generated similar GFP expression patterns and a specific and representative staining of PGCs, but injected embryos had higher rates of ectopic GFP expression (e.g. in the brain; excluded from further analysis). After enzymatic dissociation, cell suspensions were FACS-analyzed (gating as in (15)) and sorted based on their morphology and GFP intensity. After removal of aggregates (enrichment run), cells displaying the highest GFP fluorescence were all located in a narrow range on the FSC channel (purity run), indicating a morphologically homogeneous cell population. Microscopic analysis confirmed that $>95 \%$ of these isolated PGCs presented high GFP intensity (Figure S3.1). 
A
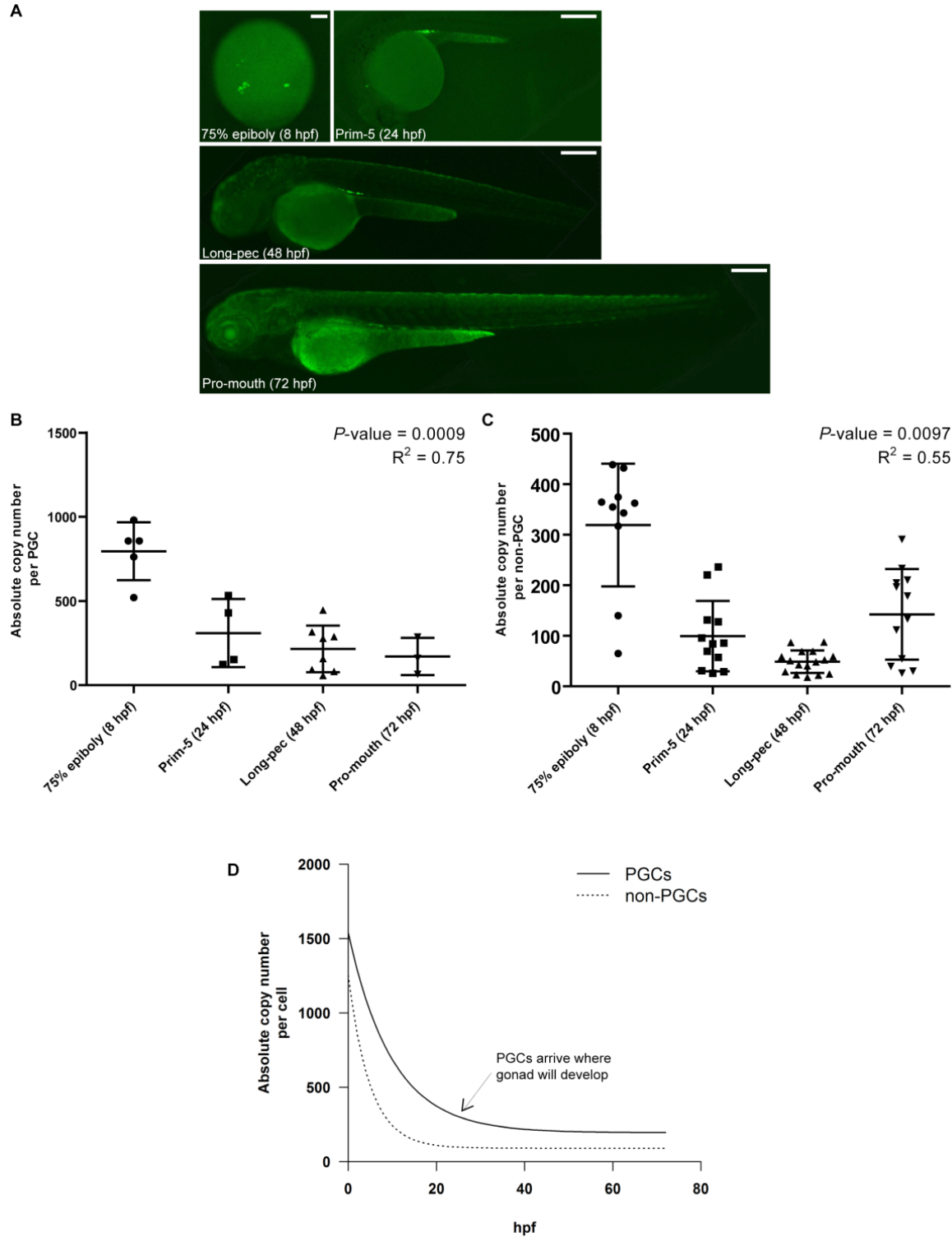

Figure 3.2: The mtDNA copy number in FACS-isolated PGCs and non-PGCs from zebrafish embryos. GFP expression in transgenic zebrafish embryos (EGFP-nanos3 3'UTR). Embryos were imaged using an Axioplan M1 Zeiss microscope. The images represent a combination of pictures captured along the anterior-posterior axis of the embryo, using specific focal planes Scale bars: $100 \mu \mathrm{m}$ for $8 \mathrm{hpf}, 200 \mu \mathrm{m}$ for 24, 48 and 72 hpf. (B-C) Absolute mtDNA copy number in (B) PGCs and (C) non-PGCs from various stages of development. Every symbol represents one group of 80 cells. Horizontal lines indicate mean with SD. P-values are generated by Spearman's rank correlation test and indicate a downward trend when $<0.05 . R^{2}$ indicates fitness of a non-linear one-phase decay exponential equation. (D) Plot of the non-linear one-phase decay equation for PGCs (derived from graph in panel B) and non-PGCS (derived from panel C). The half-life was 6.9 hpf for PGCs and $3.4 \mathrm{hpf}$ for non-PGCs. 
The germline and non-germline mtDNA bottleneck size during zebrafish embryogenesis

PGCs from embryos of 8, 24, 48 and 72 hours post fertilization (hpf) had a significantly lower mtDNA copy number compared to the oocytes (all $P$-values $<0.001$; Figure $3.2 \mathrm{~B}$ ). Although no significant differences were observed from stage 8-72 hpf (all $P$-values $>0.05$ ), a correlation analysis revealed a significant downward trend $(P$-value $=0.0009)$ in mtDNA copy number in migratory PGCs following their arrival at the region where the gonad develops (Figure 3.2D(19)). The mean values, standard deviation, range and CVs (Table S3.1) indicated that the lowest mtDNA copy number in a single PGC was $\sim 170 \mathrm{mtDNA}$ molecules at $72 \mathrm{hpf}$. The variation was high during all stages of development (Table S3.1). Correlation analysis also revealed a downward trend in the mtDNA copy number in non-PGCs during development $(P$-value $=0.0097$, Figure 3.2C). The lowest mtDNA load observed in single non-PGCs ( 50, Table S3.1) was not significantly different from the value measured for the PGCs; however both slopes (PGCs vs non-PGCs) differed statistically from each other $(P$-value $<0.0001)$. The value of $\sim 50$ is similar to the estimated non-germline bottleneck size in whole embryos. Again, variation in bottleneck size is large in non-PGCs from all developmental stages (Table S3.1). Non-linear regression analysis, based on the assumption that the mtDNA load is equally divided among the daughter cells during development, fits the measured mtDNA copy values best $\left(R^{2}=0.75\right.$ and 0.55 respectively). We estimated the plateau value ( $\approx$ bottleneck size) for PGCs at 193.5 (Standard error: 56.3 ) and for non-PGCs at 89.4 (Standard error: 16.3) mtDNA copies, which is close to the actual lowest mtDNA counts measured in this study. One phase decay equations (Figure 3.2D) indicated that the plateau value is reached earlier in non-PGCs than in PGCs, which is corroborated by the differences in the half times (6.9 hpf for PGCs and $3.4 \mathrm{hpf}$ for non-PGCs).

\section{mtDNA copy number during oocyte development}

Stage I oocytes, the primary growth phase, had an average diameter of $115 \mu \mathrm{m}$ and possessed $\sim 840,000$ mtDNA molecules (Figure 3.3A). This number was significantly higher in stage II oocytes, the cortical alveolus stage, ranging from $5.0 \times 10^{6}$ in small stage II oocytes (II small; average diameter $191 \mu \mathrm{m}$ ) to $11.1 \times 10^{6}$ in large stage II oocytes (II large; average diameter $256 \mu \mathrm{m})$. During stage III, the vitellogenesis stage, the mtDNA copy number per oocyte increased to $14.3 \times 10^{6}$ (average diameter $370 \mu \mathrm{m}$ ). Eventually, stage $\mathrm{V}$ (mature) oocytes (average diameter $750 \mu \mathrm{m}$ ) contained $17.4 \times 10^{6} \mathrm{mtDNA}$ copies. The variation was higher in stage I oocytes (CV: 106\%), compared to the other stages (CVs: 40-52\%; Table S3.1). We observed a tight positive logarithmic correlation between the volume of the oocytes stage I-III (assuming a sphere) and the mtDNA content (Figure 3.3B). 
A

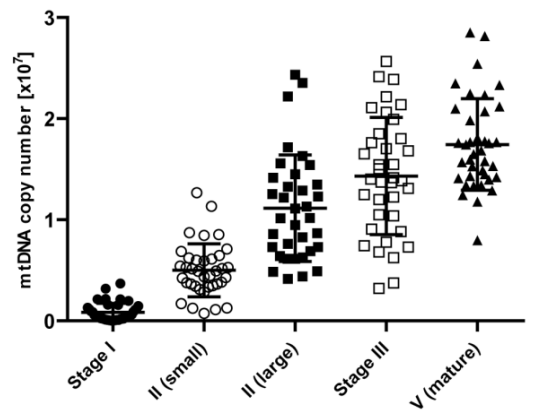

B

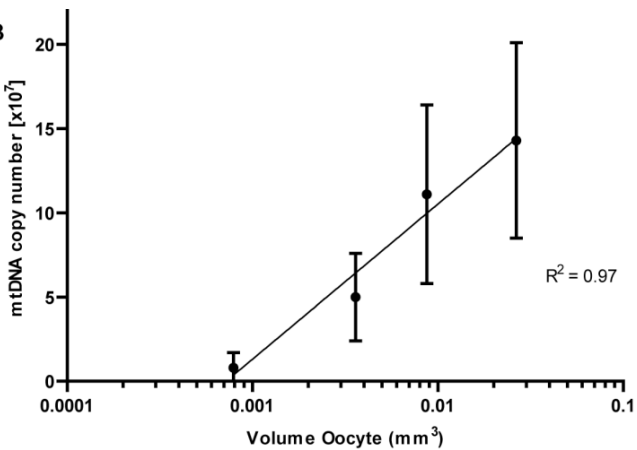

Figure 3.3: The mtDNA copy number correlates to an increased volume during oocyte maturation. (A) Mature and immature oocytes from different developmental stages were isolated from 4 different females and mtDNA copy number was measured. Each symbol represents a single (immature) oocyte. Horizontal lines indicate mean with SD. All groups were statistically different from each other. (B) Relation between the oocyte volume, assuming a sphere, and the mtDNA amount in oocytes stage I-III.. $R^{2}$ indicate fitness after a linear regression analysis between the volume (logaritmic) and the mtDNA copy number.

\section{Discussion}

High and variable mtDNA copy number in mature zebrafish oocytes

The mtDNA load in mature zebrafish oocytes was, on average, $19.0 \times 10^{6} \mathrm{mtDNA}$ copies per cell (Figure 3.1A-B). The distribution of all oocytes indicated a high, non-Gaussian variation (range: $3.3 \times 10^{6}-42.2 \times 10^{6}$ ), with a sharp lower-boundary value of $3.3 \times 10^{6}$, while the limitation on high mtDNA loads seems to be more moderate (up to $42.2 \times 10^{6}$ ). The mean oocyte mtDNA copy number among the eight different female fish was statistically similar (Figure 3.1B), suggesting low inter-individual variation. We observed variation in the DNA copy number among different batches of fish. Batch effects have been reported in zebrafish, for example, in transcriptomics (20). Our data are comparable with the reported copy number in single zebrafish blastomeres of a 4-cell stage $\left(14 \times 10^{6}\right.$ copies per cell (21)), corresponding to an mtDNA load of $56 \times 10^{6}$ in oocytes, which, although in the same order of magnitude, indicates again biological variation among batches. Oocytes from salmons, another teleost species, contain about $3.2 \times 10^{9} \mathrm{mtDNA}$ copies (22). Large differences between fish were also reported for salmon, ranging from $1.1 \times 10^{9}$ to $7.0 \times 10^{9}$ (factor $7(2)$ ).

In most mammals, mature oocytes contain less than $1.0 \times 10^{6} \mathrm{mtDNA}$ copies, ranging from 110,000 in R. Norvegicus (23) to 807,794 in B. Taurus (24), with substantial variation within species (2), e.g. in humans ranging from 143,000 (25) to 697,176 (26). For humans, mice and cows the average reported mtDNA load in the oocytes is below 300,000 copies (2), suggesting zebrafish contain about hundred times more mtDNA in their oocytes than 
mammals. This differences is most likely due to differences in implantation and in oxidative activity needed to satisfy the higher energetic demands in teleost compared to mammals due a dependency on fatty acid oxidation (22). Differences in the mtDNA load of oocytes are also reflected by differences in the size of the oocytes, which are smallest in mammals $(<0.15 \mathrm{~mm}$, human oocytes $0.1 \mathrm{~mm})$, moderate in zebrafish $(0.75 \mathrm{~mm}(16,17))$ and largest in salmons $(>4.5 \mathrm{~mm}(22))$. Based on these volumes, we estimated that the mtDNA copy number per unit of volume is fairly equal across species. This is corroborated by a study in ovine oocytes, where the mtDNA copy number per unit oocyte volume showed only little variation, much lower compared to the variation in the mtDNA copy number in the whole oocyte (27).

A selection against oocytes with low mtDNA load seems to occur (Figure 3.1A). The nonGaussian distribution of the mtDNA copy number is most likely due to a sharp boundary at the lowest mtDNA copy number. No oocytes with less than $3.3 \times 10^{6} \mathrm{mtDNA}$ copies have been observed. Mitochondrial function and copy number are important for successful fertilization (28). Low mitochondrial content has been demonstrated to negatively influence the fertilizability of eggs, probably due to inadequate mitochondrial biogenesis or cytoplasmic maturation (29), which has been demonstrated by low mtDNA counts in degenerate oocytes (30), oocytes with fertilization failure (29) or during ovarian insufficiency (31). This was confirmed by studies in mice (32). In contrast, an upper mtDNA threshold has been proposed in human blastocysts, above which implantation was never observed and aneuploidy was more frequent (33).

For each individual female fish, the inter-oocyte variation in mtDNA copy number was high ( 3 to 8 fold, Figure 3.1B). As we collected unfertilized stage $V$ (mature) oocytes (17) by squeezing the abdomen of female fish after they were kept with a male overnight, we could exclude variation in maturation of oocytes (34). Furthermore we excluded that the variation could be due to polymorphisms in the PCR primers used (Table S3.2). The considerable variation among 137 oocytes from the same eight female (Figure 3.1B) indicated that it was independent of the nuclear genome and we conclude that the variation in mtDNA copy number among oocytes is individual stochastic variation, rather than genetic variation. Such variation within an individual has also been reported in human (29) and bovine oocytes (35), which implies that every individual can generate oocytes with either a very low or a very high mtDNA copy number.

\section{mtDNA copy number per cell decreases during embryonic development}

Until the 256-cell stage, the mtDNA copy number per cell in complete zebrafish embryos halved during every cell cycle, while the total amount did not change, consistent with lack of mtDNA replication and degradation. The decrease in mtDNA content per cell continued 
until early segmentation and at these stages about 16-54 mtDNA molecules were present per cell. This is in line with findings in mouse, in which no changes in total mtDNA copy number were observed until the blastocyst stage ( 128 cells (28)). When the gastrulation stages pass into the segmentation stages, at which primary organogenesis occurs and the first muscle-driven movements can be observed (16), mtDNA replication is initiated. During this period, zebrafish embryos display maximum proton leak, which might reflect elevated mitochondrial function (36). The onset of mtDNA replication most likely reflects a switch to oxidative phosphorylation (OXPHOS) as main energy source, while the large energy requirement during the first developmental stage is provided by glycolysis (37), a faster energy source (36).

\section{High variation in the germline mtDNA bottleneck size}

In PGCs, we found a negative correlation between mtDNA copy number and developmental time with a minimal mean mtDNA copy number of 171 (an estimated 193.5 ( \pm 56.3 ) following non-linear regression analysis), with a SD of 111 . Since we quantified the mtDNA amount for groups of 80 PGCs, the actual variation among individual cells might be even higher. This high variation in PGCs from $8 \mathrm{hpf}$ to $72 \mathrm{hpf}$ is comparable to mature oocytes and the bottom of the bottleneck seems to be defined by developmental stage and not by the mtDNA copy number. Given the high variation of mtDNA copies in the oocyte, each female can produce PGCs, which, by chance, possess at the bottom only a few mtDNA molecules. At this stage, the risk that de novo mtDNA mutations (38), reach functional significance will be highest.

The germline bottleneck size of 170-200 mtDNA molecules is close to the value in mice of $\sim 185$, (based on mtDNA heteroplasmy segregation between several generations in the BALB/NZB mouse (9)) and 200 (based on direct measurements $(7,8)$ ). In humans, the bottleneck size has been estimated to be $\sim 173$, based on the heteroplasmy distribution among 82 single primary oocytes from a woman carrying the 3243A>G mtDNA mutation (39). The bottleneck size in zebrafish also resembles the size in cows (65-163 (40)), salmons (80-88 (11)) and even crickets (87-395 (40)). Some studies in humans estimated a lower bottleneck size, for instance a bottleneck size of 90 (14), only 30-35 (13), or even 1-5 (12). These were calculated from segregation patterns of variants that might not be neutral and selection events could apply (41). Since most models assume genetic drift only (42), this would lead to an underestimation of the mtDNA bottleneck size. Nevertheless, the different reported values on the bottleneck size could also reflect large biological variation. Flexibility in the decrease of mtDNA copy number during the bottleneck is in line with a mathematical model for the bottleneck (43) 


\section{Increased mtDNA copy number during oocyte maturation}

The mtDNA copy number increased rapidly during oocyte development, with a 20-fold difference between stage I oocytes and mature oocytes, indicating that mtDNA replication is a prerequisite for oocyte maturation. Substantial increase in the mtDNA copy number during early oocyte maturation has also been observed in mice (10). The correlation between the size of the cells and the mtDNA copy number (Figure 3.3B) showed that growth and mtDNA replication were closely connected. During oogenesis, a PGC of $\sim 7 \mu \mathrm{m}$ develops into an oocyte, which grows and reaches a diameter of $140 \mu \mathrm{m}$ at the end of stage I (17). At this stage variation in mtDNA copy numbers between cells was highest (ranging from $\sim 20.000$ $3.7 \times 10^{6}$ ) and no fixed oocyte volume per mtDNA could be observed. Probably, at this stage cell growth precedes and might trigger mtDNA replication, whereas during the further developmental stages, it is less clear whether cell growth drives mtDNA replication or vice versa. We failed to measure an increase in mTDNA copy number in PGCs that arrived at the region where the gonads develop, as has been described in mice $(7,8)$. This discrepancy might arise because fish oogonia, in contrast to mammals, keep constantly renewing the stocks of young oocytes and follicles. In fish, mitosis in oogonia (starting point of oogenesis) is activated after ovulation in adult zebrafish (44), while human oogonia undergo mitosis already during weeks 9-22 of embryonic development (45). Although we were not able to isolate oogonia and the earliest and smallest primary oocytes, the high mtDNA copy numbers in stage I oocytes suggests that the mtDNA replication necessary to achieve high oocyte mtDNA loads occurred later in zebrafish life (in adults) than in mammals (during embryonic development).

\section{A small non-germline mtDNA bottleneck size}

The bottleneck size in non-germline zebrafish cells (non-PGCs) is $~ 50$ mtDNA molecules (an estimated $89.4 \pm 16.3$ following non-linear regression analysis) per cell with a downward trend in the mtDNA copy number during development. As non-PGCs can be any cell from the developing embryo, the bottleneck size derived from the whole embryo analysis (1654 mtDNA copies) confirms this number. The decrease in mtDNA copy number has been suggested to establish a set-point during early embryogenesis, which increases later during development in order to satisfy cell-specific ATP requirements through OXPHOS (46). During the first $48 \mathrm{hpf}$ we did not observe an increase in the mtDNA copy number of the nongermline cells (Figure 3.1), while after $72 \mathrm{hpf}$ a trend towards higher mtDNA amounts was observed (Figure 3.2C). The latter observation could indicate that mtDNA amounts go up once the larvae use their skeletal muscle for free swimming and hunting (16). 


\section{The germline and the non-germline mtDNA bottleneck}

In line with our observation in zebrafish, murine somatic cells have highly variable mtDNA copy numbers during early embryogenesis and the mtDNA content was downregulated from $8 \mathrm{dpc}$ onwards (10). Furthermore, as in the present study, the mtDNA copy number in murine non-germline cells was lower than in germline cells (10). Although mtDNA copy number between PGCs and non-PGCs were not statistically different, correlation and nonlinear regression analysis (Figure 3.2C) indicated a difference in the course or timing of the mtDNA copy number over development. The increase in mtDNA content in germline cells occurs during oogenesis in adult zebrafish (Figure 3.4), long after the formation of the organ systems (non-germline cells) during zebrafish embryogenesis. This difference in timing, if comparable in humans, could explain differences in segregation of mtDNA heteroplasmic mutations among germline and somatic tissues in humans (42). Especially in case of familial mtDNA mutations (2), these segregation and replication mechanisms could explain the large tissue-specific differences in mutation load (47). This is corroborated by the observation that HIV-interventions, which reduce mtDNA copy number can induce a somatic bottleneck, can result in age-related mitochondrial disease due to clonal expansion of preexisting mtDNA mutations (48).

We have quantified the whole germline mtDNA bottleneck in a single model (Figure 3.4). Mechanistically, both the germline and the non-germline bottlenecks rely on the same principle: division of the mtDNA over the daughter cells during early embryogenesis in the absence of replication, until increased energy requirements during development or oogenesis activate mtDNA replication. However, as the bottleneck in non-germline cells only affects the individual, while segregation in PGCs affects future generations, the germline bottleneck is of higher evolutionary importance. Nevertheless, an understanding of the difference between the bottlenecks would allow a better prediction of the risk an individual has to develop an mtDNA disorder, which is determined by both the germline and the non-germline bottleneck. Due to a clear difference in somatic and germline specification in zebrafish, as well as fast development and easy access to the organs, zebrafish are an attractive model to study these possible tissue-specific mtDNA bottleneck effects in further detail. 


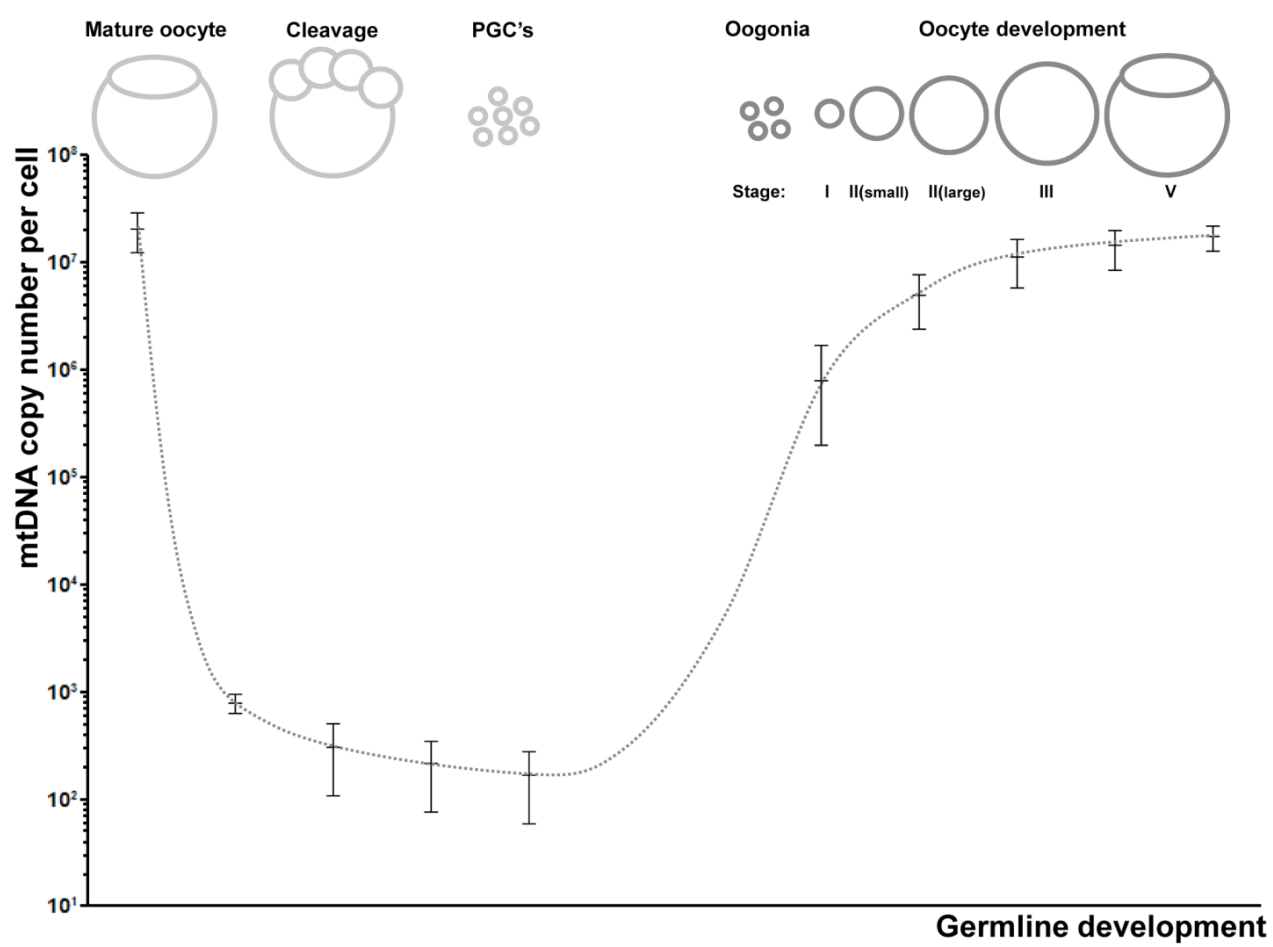

Figure 3.4: Summary of the germline mtDNA bottleneck. The germline mtDNA bottleneck consists of 1) random partitioning of oocyte mtDNA during the early developmental cell divisions, leading to variable mtDNA bottleneck sizes and 2) extensive mtDNA replication during oogenesis.

\section{Acknowledgements}

R. Stephan and S Ormenese (GIGA Flow Cytometry platform, Liège University, Belgium) are acknowledged for their assistance during cell sorting, I. Eijkenboom for generating the mt-nd1 curves, M. Adriaens for reviewing statistical analyses, R. Goto-Kazeto (Hokkaido University, Japan) for supplying the nanos3 3'UTR pCS2+ vector and Jana Pfeiffer (Münster University, Germany) for assisting in transportation of the transgenic zebrafish. We are grateful for the fellowship of GROW-School for oncology and Developmental Biology to A.B.C.O. and H.J.M.S., to the alma-in-silico project funded by the Interreg IV program of the EC to M.M. and H.J.M.S., the E-RARE 2 project GENOMIT [R 50.02.12F] to M.M. and H.J.M.S. and to the Dutch Princes Beatrix Fonds for their grant to T.E.J.T. and H.J.M.S.. K.T. and E.R. are supported by the European Research Council (ERC), the Deutsche Forschungsgemeintschaf (DFG) and the Cells in Motion cluster of Excellence (EXC 1003-CIM). M.W. and M.M. are grateful to Walloon Region (FEDER. GIGA2 Bioindustry support) and the Fond Nacional de la Recherché Scientifique (F.N.R.S.) with the GENOMIT project. M.G. and H.J.M.S. are grateful for the support of the Dutch Province of Limburg. 


\section{References}

1. Lynch M, Koskella B, Schaack S. Mutation pressure and the evolution of organelle genomic architecture. Science (New York, NY). 2006;311(5768):1727-30.

2. Otten $A B, S m e e t s ~ H J$. Evolutionary defined role of the mitochondrial DNA in fertility, disease and ageing. Hum Reprod Update. 2015.

3. Lee HS, Ma H, Juanes RC, Tachibana M, Sparman M, Woodward J, et al. Rapid mitochondrial DNA segregation in primate preimplantation embryos precedes somatic and germline bottleneck. Cell Rep. 2012;1(5):506-15.

4. Sharpley MS, Marciniak C, Eckel-Mahan K, McManus M, Crimi M, Waymire K, et al. Heteroplasmy of mouse mtDNA is genetically unstable and results in altered behavior and cognition. Cell. 2012;151(2):333-43.

5. Howell N, Halvorson S, Kubacka I, McCullough DA, Bindoff LA, Turnbull DM. Mitochondrial gene segregation in mammals: is the bottleneck always narrow? Hum Genet. 1992;90(1-2):117-20.

6. Hauswirth WW, Laipis PJ. Mitochondrial DNA polymorphism in a maternal lineage of Holstein cows. Proc Natl Acad Sci U S A. 1982;79(15):4686-90.

7. Cree LM, Samuels DC, de Sousa Lopes SC, Rajasimha HK, Wonnapinij P, Mann JR, et al. A reduction of mitochondrial DNA molecules during embryogenesis explains the rapid segregation of genotypes. Nat Genet. 2008;40(2):249-54.

8. Wai T, Teoli D, Shoubridge EA. The mitochondrial DNA genetic bottleneck results from replication of a subpopulation of genomes. Nat Genet. 2008;40(12):1484-8.

9. Jenuth JP, Peterson AC, Fu K, Shoubridge EA. Random genetic drift in the female germline explains the rapid segregation of mammalian mitochondrial DNA. Nat Genet. 1996;14(2):14651.

10. Cao L, Shitara H, Horii T, Nagao Y, Imai H, Abe K, et al. The mitochondrial bottleneck occurs without reduction of mtDNA content in female mouse germ cells. Nat Genet. 2007;39(3):38690.

11. Wolff JN, White DJ, Woodhams M, White HE, Gemmell NJ. The strength and timing of the mitochondrial bottleneck in salmon suggests a conserved mechanism in vertebrates. PLoS One. 2011;6(5):e20522.

12. Marchington DR, Hartshorne GM, Barlow D, Poulton J. Homopolymeric tract heteroplasmy in mtDNA from tissues and single oocytes: support for a genetic bottleneck. Am J Hum Genet. 1997;60(2):408-16.

13. Rebolledo-Jaramillo B, Su MS, Stoler N, McElhoe JA, Dickins B, Blankenberg D, et al. Maternal age effect and severe germ-line bottleneck in the inheritance of human mitochondrial DNA. Proc Natl Acad Sci U S A. 2014;111(43):15474-9.

14. Pallotti $F$, Binelli $G$, Fabbri $R$, Valentino $M L$, Vicenti $R$, Macciocca $M$, et al. A wide range of $3243 \mathrm{~A}>\mathrm{G} / \mathrm{tRNALeu}(\mathrm{UUR})$ (MELAS) mutation loads may segregate in offspring through the female germline bottleneck. PLoS One. 2014;9(5):e96663.

15. Goto-Kazeto R, Saito T, Takagi M, Arai K, Yamaha E. Isolation of teleost primordial germ cells using flow cytometry. The International journal of developmental biology. 2010;54(10):1487-92.

16. Kimmel CB, Ballard WW, Kimmel SR, Ullmann B, Schilling TF. Stages of embryonic development of the zebrafish. Developmental dynamics : an official publication of the American Association of Anatomists. 1995;203(3):253-310.

17. Selman K, Wallace RA, Sarka A, Qi X. Stages of oocyte development in the zebrafish, Brachydanio rerio. Journal of Morphology. 1993;218(2):203-24.

18. Otten AB, Stassen AP, Adriaens M, Gerards M, Dohmen RG, Timmer AJ, et al. Zebrafish oocytes with a low mtDNA copy number are at risk of de novo mtDNA point mutations reaching functional significance (chapter 4). Thesis, Auke Otten. 2016. 
19. Paksa A, Raz E. Zebrafish germ cells: motility and guided migration. Curr Opin Cell Biol. 2015;36:80-5.

20. Wang RL, Bencic DC, Garcia-Reyero N, Perkins EJ, Villeneuve DL, Ankley GT, et al. Natural Variation in Fish Transcriptomes: Comparative Analysis of the Fathead Minnow (Pimephales promelas) and Zebrafish (Danio rerio). PLoS One. 2014;9(12):e114178.

21. Artuso L, Romano A, Verri T, Domenichini A, Argenton F, Santorelli FM, et al. Mitochondrial DNA metabolism in early development of zebrafish (Danio rerio). Biochim Biophys Acta. 2012;1817(7):1002-11.

22. Wolff JN, Gemmell NJ. Lost in the zygote: the dilution of paternal mtDNA upon fertilization. Heredity. 2008;101(5):429-34.

23. Zeng HT, Yeung WS, Cheung MP, Ho PC, Lee CK, Zhuang GL, et al. In vitro-matured rat oocytes have low mitochondrial deoxyribonucleic acid and adenosine triphosphate contents and have abnormal mitochondrial redistribution. Fertility and sterility. 2009;91(3):900-7.

24. Iwata H, Goto H, Tanaka H, Sakaguchi Y, Kimura K, Kuwayama T, et al. Effect of maternal age on mitochondrial DNA copy number, ATP content and IVF outcome of bovine oocytes. Reproduction, fertility, and development. 2011;23(3):424-32.

25. Duran HE, Simsek-Duran F, Oehninger SC, Jones HW, Jr., Castora FJ. The association of reproductive senescence with mitochondrial quantity, function, and DNA integrity in human oocytes at different stages of maturation. Fertility and sterility. 2011;96(2):384-8.

26. Murakoshi Y, Sueoka K, Takahashi K, Sato S, Sakurai T, Tajima H, et al. Embryo developmental capability and pregnancy outcome are related to the mitochondrial DNA copy number and ooplasmic volume. Journal of assisted reproduction and genetics. 2013;30(10):1367-75.

27. Cotterill M, Harris SE, Collado Fernandez E, Lu J, Huntriss JD, Campbell BK, et al. The activity and copy number of mitochondrial DNA in ovine oocytes throughout oogenesis in vivo and during oocyte maturation in vitro. Molecular human reproduction. 2013;19(7):444-50.

28. Ebert KM, Liem H, Hecht NB. Mitochondrial DNA in the mouse preimplantation embryo. Journal of reproduction and fertility. 1988;82(1):145-9.

29. Reynier P, May-Panloup P, Chretien MF, Morgan CJ, Jean M, Savagner F, et al. Mitochondrial DNA content affects the fertilizability of human oocytes. Molecular human reproduction. 2001;7(5):425-9.

30. Santos TA, El Shourbagy S, St John JC. Mitochondrial content reflects oocyte variability and fertilization outcome. Fertility and sterility. 2006;85(3):584-91.

31. May-Panloup P, Chretien MF, Jacques C, Vasseur C, Malthiery $Y$, Reynier P. Low oocyte mitochondrial DNA content in ovarian insufficiency. Human reproduction. 2005;20(3):593-7.

32. Wai T, Ao A, Zhang X, Cyr D, Dufort D, Shoubridge EA. The role of mitochondrial DNA copy number in mammalian fertility. Biology of reproduction. 2010;83(1):52-62.

33. Fragouli E, Spath K, Alfarawati S, Kaper F, Craig A, Michel CE, et al. Altered levels of mitochondrial DNA are associated with female age, aneuploidy, and provide an independent measure of embryonic implantation potential. PLoS genetics. 2015;11(6):e1005241.

34. Jansen RP, de Boer K. The bottleneck: mitochondrial imperatives in oogenesis and ovarian follicular fate. Molecular and cellular endocrinology. 1998;145(1-2):81-8.

35. Cree LM, Hammond ER, Shelling AN, Berg MC, Peek JC, Green MP. Maternal age and ovarian stimulation independently affect oocyte mtDNA copy number and cumulus cell gene expression in bovine clones. Human reproduction. 2015;30(6):1410-20.

36. Stackley KD, Beeson CC, Rahn JJ, Chan SS. Bioenergetic profiling of zebrafish embryonic development. PLoS One. 2011;6(9):e25652.

37. Facucho-Oliveira JM, St John JC. The relationship between pluripotency and mitochondrial DNA proliferation during early embryo development and embryonic stem cell differentiation. Stem cell reviews. 2009;5(2):140-58. 
38. Thorburn DR. Mitochondrial disorders: prevalence, myths and advances. J Inherit Metab Dis. 2004;27(3):349-62.

39. Brown DT, Samuels DC, Michael EM, Turnbull DM, Chinnery PF. Random genetic drift determines the level of mutant mtDNA in human primary oocytes. Am J Hum Genet. 2001;68(2):533-6.

40. Rand DM, Harrison RG. Mitochondrial DNA transmission genetics in crickets. Genetics. 1986;114(3):955-70.

41. Stewart JB, Freyer C, Elson JL, Wredenberg A, Cansu Z, Trifunovic A, et al. Strong purifying selection in transmission of mammalian mitochondrial DNA. PLoS biology. 2008;6(1):e10.

42. Wonnapinij P, Chinnery PF, Samuels DC. The distribution of mitochondrial DNA heteroplasmy due to random genetic drift. Am J Hum Genet. 2008;83(5):582-93.

43. Johnston IG, Burgstaller JP, Havlicek V, Kolbe T, Rulicke T, Brem G, et al. Stochastic modelling, Bayesian inference, and new in vivo measurements elucidate the debated mtDNA bottleneck mechanism. eLife. 2015;4:e07464.

44. Jalabert B. Particularities of reproduction and oogenesis in teleost fish compared to mammals. Reproduction, nutrition, development. 2005;45(3):261-79.

45. Jones RE. Human Reproductive Biology. San Diego: Academic Press, Elsevier; 1997.

46. Facucho-Oliveira JM, Alderson J, Spikings EC, Egginton S, St John JC. Mitochondrial DNA replication during differentiation of murine embryonic stem cells. Journal of cell science. 2007;120(Pt 22):4025-34.

47. St John JC. Transmission, inheritance and replication of mitochondrial DNA in mammals: implications for reproductive processes and infertility. Cell and tissue research. 2012;349(3):795808.

48. Payne BA, Wilson IJ, Hateley CA, Horvath R, Santibanez-Koref M, Samuels DC, et al. Mitochondrial aging is accelerated by anti-retroviral therapy through the clonal expansion of mtDNA mutations. Nat Genet. 2011;43(8):806-10.

\section{Supplementary}

Supplementary Figures, Experimental Procedures and tables are available online at http:// dx.doi.org/10.1016/j.celrep.2016.06.023 


\section{CHAPTER 8}

General Discussion 
Chapter 8 


\section{Finding the genetic cause}

\section{WES}

As illustrated in chapter 2, WES is a successful approach to identify the genetic defect in complex mitochondrial disease, as WES of our heterogeneous, but well-characterized patient cohort solved $69 \%$ of the cases. This included patients who were likely to have an autosomal recessive inherited gene defect (consanguineous and/or multiple affects siblings) and single non-consanguineous patients, where a de novo mutation could possibly underlie the disease manifestation. As we did not perform WES in the parents of single non-consanguineous patients (trio-analysis), but only checked the clinical exome for dominant mutations, we might have missed part of the de novo gene defects. Finally, we also identified possible genetic causes in unknown genes, which required functional validation. Therefore, the overall yield will most likely become even higher. Due to limited genotype-phenotype correlations and, as a result, the limited success rate of gene panels, WES is the preferred starting method to identify the genetic defect in both consanguineous and non-consanguineous families with heterogeneous mitochondrial disease. It is an unbiased approach, interrogating all genetic variants in the patient's exome. Especially, in consanguineous families, which tend to carry more genetic defects than outbred families, a complete genetic analysis is crucial to reveal multi-genic disease causes, as shown in chapter 3. A direct consequence of a genetic diagnosis is the possibility to prevent further transmission of the disease and get an unaffected child by either prenatal genetic diagnosis (PND) or Preimplantation Genetic Diagnosis (PGD) (1). Additionally, in consanguineous families, WES can be useful to genetically screen couples for their risk of having a child with a recessive genetic disease at a preconception stage. Preconception carrier screening (PCS) can characterize the carrier status and therewith identify couples that have a $25 \%$ risk, or higher in case of multiple gene defects, of having an affected child. PCS will provide these couples with informed reproductive choices such as PND, PGD, or accepting the genetic risk (2).

In comparison to targeted sequencing methods or the application of virtual gene panels, we have demonstrated that WES is much more successful. Up to $30 \%$ of the disease-causing mutations identified in our patient cohort were not present in the MitoCarta database at the time of discovery, and would have been missed with a panel based analysis. This includes novel genes, but also genes with a reported non-mitochondrial disease manifestation. Besides, working with gene panels will require continues updating and can easily result in the sequential use of different, partly overlapping gene panels one after another. Whole genome sequencing (WGS) does have several advantages above WES as it results in a better 
overall coverage of the exonic regions, includes all genes, and does not cause artifacts due to gene enrichment $(3,4)$. Also, it allows the detection of translocations and intronic variants that could affect gene function via alternative splicing or regulatory microRNAs (5). Currently, the main disadvantage of WGS is the higher cost compared to WES (6). However, if in future the WGS costs will drop, it will rapidly become the new standard for genetic diagnosis. Transcriptome analysis by RNASeq has been shown to be a powerful diagnostic tool, especially in combination with WES or WGS, as it is a quantitative method that can identify aberrantly expressed genes, aberrant splicing events or mono-allelic expressed variants, and is able to solve an additional $10 \%$ of the undiagnosed mitochondrial patients (7). Preferably, transcriptome sequencing should follow WES to solve the unresolved cases or to be applied together with WGS to interpret the intronic variants that are currently still difficult to judge based on available in silico splice-prediction tools $(7,8)$. Although metabolic (mitochondrial) genes are expressed in most tissues, one should be aware that genetic defects might be missed due to tissue specific mRNA expression patterns, it is therefore important to isolate the transcriptome from patient tissues that are phenotypically affected and to have proper "healthy" control samples available as a reference for the gene expression levels and isoforms that are naturally present.

\section{Genetic models}

Novel mutated genes or novel variants might not be unambiguously defined as the cause of disease by current approaches and may require additional, functional validation. In the case of WES, there is demand for a genetic model that allows the testing of variant pathogenicity in a relatively quick and straightforward manner, specifically for variants of unknown significance (VUS) and variants in novel genes. Suitable read-out models to test variant pathogenicity in mitochondrial disease can be based on in silico analysis, in vitro testing (e.g. patient cells or IPSCs) or in vivo models.

\section{1) In silico analysis}

In-depth in silico analysis can be a valuable tool to estimate the impact of a mutation on the protein its function. Protein crystal structure based modelling can help to predict the consequences of an amino-acid substitution on protein folding, where alterations in secondary or tertiary protein structure, which can result from changes in hydrophobicity, polarity or size of the amino-acid residue, can be simulated (9-11). Also, to interpret the effect of a mutation on the protein-protein interaction, crystal structure based modeling and protein docking tools can be useful, as illustrated in chapter 4 by the likely affected 
CLPP-CLPX interaction in a patient with Perrault syndrome. Another promising field that is quickly developing is systems biology, where measuring and mapping of biological processes or functions within cells, organelles, tissues, organs, or organ systems can help to predict how biological processes that consist of many interacting components behave, and how a specific protein defect can influence this process. Several papers have been published on mitochondrial physiology, and have shown interesting new findings (12-14). Modeling of the mitochondrial respiratory system can help to predict the consequences of a genetic defect at the metabolic level, where estimated changes in enzyme activity, substrate consumption or metabolites can generate valuable information to define precise read-out parameters for further laboratory testing. Although, in silico analysis can give important information on the impact of a variant on protein structure, protein interactions or even a complete metabolic process, these should be functionally validated before being used as a prediction tool in the clinic.

\section{2) In vitro models}

Extensive phenotypic profiling of the patient in terms of clinical symptoms, metabolic and biochemical measurements is crucial, not only to select candidate genes during filtering of WES data, but also to establish a suitable read-out model that can be used to link the genetic defect to the patient's phenotype. Complementation studies in patient derived cells, which express the candidate gene and exhibit a biochemical, metabolic, or morphological disease phenotype, are a relatively quick and commonly used approach to link a genetic defect to the patient's phenotype $(15,16)$. In these studies, the introduction of the wild-type candidate gene is expected to restore the disease phenotype at the cellular level, whereas the introduction of the mutated gene will have no effect. As shown in chapter 2, for most of our mitochondrial patients, fibroblasts are available and can be successfully used to perform complementation studies based on lentiviral transduction system or transient transfection (less effective in fibroblasts). Yet, fibroblasts are not always suitable as a read-out model for respiratory defects, as OXPHOS deficiencies can sometimes be relatively mild compared to muscle or nerve tissue due to a lower OXPHOS metabolism with less mitochondria per cell. Mitochondrial respiratory parameters such as oxygen consumption and ATP production (GlowMax, Oroboris, Seahorse system), complex activity (Oroboris, In-gel activity), OXPHOS complex assembly (Blue-native PAGE) or mitochondrial network integrity (MitoTracker) are frequently used read-out systems to test for primary mitochondrial disease (17-19).

Tissue-specific gene expression, for example restricted to muscle or neurons, can complicate the testing of variant pathogenicity (e.g. splice variants, RNA and protein expression levels and localization). Patient tissue-biopsies are often not available and difficult to maintain in 
cell culture (post-mitotic tissues). Induced reprogramming of any available patient cell-type, e.g. fibroblast towards IPSC's, will subsequently allow the differentiation of IPSCs towards any cell type, maintaining the patient's unique genetic background. Besides, differentiation of IPSCs towards muscle or neuronal progenitor cells, which are still in a multi-potent state (e.g. mesoangioblasts or neuroblasts, respectively) allows culturing of these adult stem cells to a high number of passages, facilitating the testing in an in vitro setting (20-22). A limitation of IPSC reprogramming is the heterogeneity within an IPSC population, being diverse in differentiation capacity (23). Also when studying the impact of mtDNA defects, IPSC reprogramming is not always a suitable approach as mtDNA heteroplasmy levels may change or even disappear $(24,25)$, probably due to a segregational bottleneck (as described in chapter 7), and/or via active mtDNA selection or degradation mechanisms (26). To investigate the influence of heteroplasmic or homoplasmic mtDNA mutations on the cellular disease phenotype, cybrid cells are a commonly used tool. Cybrids allow the evaluation of the patient's mtDNA in an isolated manner, as the nDNA background of the patient is replaced by a 'healthy' nuclear genome from a donor cell. Cybrid cells can demonstrate unambiguously that the defect is located in the mtDNA or nuclear genes. Cybrids are produced by combining $\rho 0$ cells, containing a nucleus but no mtDNA (mtDNA depletion is induced by exposure to ethidium bromide), with cytoplasts from the patient, containing the mtDNA (nucleus is removed by ultracentrifugation)(27-29).

\section{3) In vivo models}

The development of novel, highly powerful tools to perform genetic manipulation, such as CRISPR/Cas9 and gene therapy, has significantly improved the opportunities to create animal models to study genetic disease. As each specific animal model has its advantages and disadvantages, it is highly dependent on the research question, which animal model is chosen. No single model exists that fits all. A major criterion when selecting a suitable animal model is that the biological process, in which the gene of interest is involved, should be evolutionarily conserved and therefore is comparable to the human situation. Genetically simple animal models such as yeast can especially be beneficial when studying such processes at the biochemical level, but do not suffice for studying the pathophysiology. On the other hand, mice are genetically and physiologically much closer to humans and would be more suitable to study disease symptom manifestations. Other factors that define the suitability of an animal model include the applicability and efficiency to induce genetic modifications and the time and costs involved (Table 1). 
Table 1: Animal models for mitochondrial disease with their advantages and disadvantages.

\begin{tabular}{|c|c|c|}
\hline animal model & advantages & disadvantages \\
\hline Yeast & $\begin{array}{ll}\text { - } & \text { small and simple genome } \\
\text { - } & \text { short life span } \\
\text { - } & \text { cheap in maintenance } \\
\text { - } & \text { easy to manipulate genetically } \\
\text { - } & \text { systerates drastic mutations in OXPHOS loss of mtDNA } \\
\text { mtDNA heteroplasmy levels can be } \\
\text { manipulated via biolistic particle } \\
\text { delivery }\end{array}$ & $\begin{array}{l}\text { - } \quad \text { genetically/evolutionarily distant from } \\
\text { humans } \\
\text { not suitable to study phenotypical } \\
\text { aspects such as symptom } \\
\text { manifestations; only for fundamental } \\
\text { molecular processes } \\
\text { part of the OXPHOS complexes have a } \\
\text { different composition than in humans; } \\
\text { respiratory super complexes are } \\
\text { differently arranged }\end{array}$ \\
\hline Drosophila & $\begin{array}{l}\text { - } \quad \text { compact genome size } \\
\text { - } \quad \text { short developmental time and life span } \\
\text { - } \quad \text { allows to study disease phenotypes at } \\
\text { the clinical and molecular level } \\
\text { - } \quad \text { model for behavioral studies; some } \\
\text { complex behaviors are comparable to } \\
\text { humans } \\
\text { genome editing and silencing } \\
\text { techniques such as siRNA's, CRISPR/ } \\
\text { Cas9 and gene therapeutics can be } \\
\text { applied }\end{array}$ & $\begin{array}{l}\text { as invertebrate model, drosophila } \\
\text { lacks some of the organ systems and } \\
\text { structures that are important in human } \\
\text { disease } \\
\text { more difficult to perform gene editing } \\
\text { than in yeast, especially to modify } \\
\text { mtDNA heteroplasmy levels }\end{array}$ \\
\hline Zebrafish & $\begin{array}{l}\text { - } \quad \text { vertebrate animal model, relying on } \\
\text { many of the same organs as human } \\
\text { nuclear genome size half of mammalian } \\
\text { genome; mtDNA highly similar to } \\
\text { humans } \\
\text { - low costs in maintenance } \\
\text { high numbers of offspring, transparent } \\
\text { during embryonic development, } \\
\text { develop fast and outside the body in } \\
\text { the water } \\
\text { suitable model to study development } \\
\text { of mitochondrial disease, mtDNA } \\
\text { transmission, and behavior } \\
\text { transgenesis based on transposons is } \\
\text { possible } \\
\text { TALENs, CRISPR/Cas9 and gene therapy } \\
\text { can be applied } \\
\text { Morpholino injection at } 1 \text {-cell stage } \\
\text { is a quick and easy method for gene } \\
\text { silencing }\end{array}$ & $\begin{array}{l}\text { - have many genes duplicated, can be } \\
\text { difficult to identify human orthologues } \\
\text { Morpholinos last only during embryonic } \\
\text { development (3-5 days) and can have } \\
\text { toxic side effects } \\
\text { more difficult to perform gene editing } \\
\text { than in yeast, especially to modify } \\
\text { mtDNA heteroplasmy levels }\end{array}$ \\
\hline Mice & $\begin{array}{l}\text { - } \\
\text { vertebrate mammalian model } \\
\text { closest to humans with regard to } \\
\text { development, genetic profile and } \\
\text { physiological processes } \\
\text { CRISPR/Cas9 and gene therapy can be } \\
\text { applied }\end{array}$ & $\begin{array}{l}\text { - labor intensiveness, high costs } \\
\text { slow development, requiring significant } \\
\text { amount of time to create genetic knock- } \\
\text { out or mutant models } \\
\text { - } \quad \text { more difficult to obtain large numbers } \\
\text { and statistical power than with fly or } \\
\text { fish models } \\
\text { manipulation of mtDNA is still } \\
\text { challenging }\end{array}$ \\
\hline
\end{tabular}


- Yeast

Much of our current knowledge of mitochondrial function and pathology is derived from yeast studies, especially from Saccharomyces cerevisiae. As yeast has fermenting capacity, it can survive mutations that drastically inactivate the OXPHOS system and can tolerate loss of mtDNA, allowing the studying of 'extreme' mitochondrial defects in a relatively simple setting. Yeast has a small genome, is relatively easy to genetically manipulate, and has a short life span. These characteristics make it an excellent model to study the molecular basis of mitochondrial disease (30). Yeast models have been extensively used to study OXPHOS complex assembly and the influence of both mtDNA and nDNA encoded defects on this process. However, part of the OXPHOS complexes have a different composition than in humans, and respiratory supercomplexes are differently arranged in the mitochondrial inner-membrane (31). S. cerevisiae has been utilized as a good model to study mtDNA mutations that are identified in patients, as mtDNA heteroplasmy levels can be manipulated in a controlled manner via biolistic particle delivery. With this method in vitro generated mtDNA fragments, containing a specific mutation of interest, can be directly transfected into mitochondria of the yeast model using DNA-coated gold particles. Integration of these fragments into wild-type mtDNA via homologous DNA recombination will generate heteroplasmic mtDNA conditions (32). As yeast is unable to maintain heteroplasmic mtDNA levels, homoplasmic mtDNA populations can be relatively easy obtained (33). In this manner, for a variety of pathogenic mtDNA mutations the functional consequences have been unraveled, including mutations in genes encoding subunits of complexes III (cytochrome $b$ ) and IV (COXI, COXIII) $(34,35)$, and in mt-tRNA genes $(36,37)$. Since the discovery of the first nDNA defect causing mitochondrial disease (38), over 150 nuclear genes have been identified that are associated with mitochondrial disease (39-41). 70\% of these genes are conserved in yeast (30), indicating that despite its small genome, there are significant genetic similarities between yeast and human mitochondria that can help to characterize the genetic and biochemical causes of nDNA encoded mitochondrial disease. For example, in the C173 and W125 mutants of S. cerevisiae, which are partially deficient in cytochrome oxidase and have a respiratory deficiency, complementation with the yeast homolog of the mammalian SURF-1 gene, was found to restore the respiratory deficiency. The latter showing that the SHY1 gene is the yeast homolog of the mammalian SURF-1 gene, encoding a mitochondrial complex IV assembly factor required for respiration $(42,43)$.

\section{- Drosophila}

Drosophila has been successfully used as a model system to study disease phenotypes at the clinical and molecular level in neurological and mitochondrial disorders $(44,45)$. Around $70 \%$ of the human disease genes are conserved in the fly $(46,47)$. For example, Parkinsons Disease genes were studied in Drosophila melanogaster for their role in fundamental 
biological processes, such as cell death $(48,49)$, cell proliferation, growth, and migration (50). Also at the behavioral level, flies display complex behavior comparable to humans, such as circadian rhythm, learning and memory, sleep, and aggression (50), suggesting that the genetic basis of these behaviors could be conserved from fly to man. An advantage of Drosophila is the compact genome size, being approximately $1 / 30$ th of the human genome and can be easily modified using some of the recent developed genome editing techniques, like CRISPR/Cas9, and gene therapy strategies (51-53). Also the short developmental time of 10 days and life span of 60-80 days allows relatively quick generation of mutant models and include siRNA based methods for temporary gene silencing $(54,55)(56)$. These features make flies well suited to study the function of neurodegenerative and mitochondrial diseases genes $(57,58)$. A key advantage is the possibility to generate homoplasmic mtDNA models, by manipulating the Drosophila mtDNA with targeted restriction enzymes (59). The creation of a homoplasmic ND2 mutant served as an animal model of complex-I-associated disease and exhibited phenotypic symptoms comparable to complex I deficiency in humans, including stress-induced seizures, progressive neurodegeneration and shortened lifespan (60). Yet, being invertebrates, the drosophila lacks organ systems and structures that are involved in certain human disease and therefore the model is limited to those organ systems which do match. Drosophila can also be used to screen chemical compounds, as these can be administered to the food in a precisely defined concentration and the animals can be phenotypically screened in large numbers (61).

\section{- Zebrafish}

A highly suitable vertebrate animal model to study mitochondrial disease and the transmission of mtDNA towards offspring is the zebrafish. Zebrafish rely on many of the same organs as human, however they produce high numbers of offspring that are fully transparent during embryonic development, which occurs outside of the body. This makes the analysis of morphological alterations and tissue pathology during development easy (62). Embryonic cells can be analyzed from the moment of fertilization, as illustrated in chapter 7 by our mtDNA copy numbers quantifications $(63,64)$. Zebrafish contain a nuclear genome, which is half the size of other mammals (65), and the mtDNA has high identity to human mtDNA with the same composition of 37 genes (66). Reverse genetic approaches in zebrafish modelling rely on the knock-out, silencing (knockdown) or mutagenesis of nuclear genes, mainly to induce a loss of function phenotype by targeting genes in which human disease-causing mutations have been identified. This strategy was shown successful in studying pathology, using techniques such as zinc-finger nucleases (67), TALENs (68) and most recently CRISPR/ Cas9 (69). However, zebrafish have approximately $20-30 \%$ of the genes duplicated $(70,71)$, making it sometimes difficult to identify the orthologues of human genes and to generate full knock-out or mutated models (72). The use of anti-sense morpholinos, injected at the 
1-cell stage is a rapid strategy to induce temporary gene silencing (knockdown). Both splicesite, inducing aberrant splicing, and translation morpholinos, which block mRNA translation within the ribosomes have been used (73). A disadvantage of morpholinos is the fact that mRNA silencing lasts only for 3-5 days (during embryonic development), only approximately $1 / 3$ of the morpholinos is effective, and non-specific toxicity can be observed. Also the injection of mutated human mRNA (if sufficient homologous to the fish mRNA) is often used to assess the effects of a specific mutation, either combined with morpholino-based gene silencing (the wild-type gene) or just by overexpression (74). Morpholino and mRNA injections can be applied in a high-throughput manner using robotic injection systems, followed by fully automated analysis of phenotypic parameters, combined allowing rapid screening of the many different candidate mutations that result from WES analysis (75-77). Transgenesis has also been successfully applied in zebrafish. A commonly used transgenesis method is based on DNA transposons with the Tol2 system, a robust gene transfer tool to generate transgenic zebrafish lines with the possibility to visualize or manipulate specific tissues (78). As in yeast and Drosophila, large groups of animal models can be generated to obtain sufficient statistical power. Numerous assays have been developed to study behavioral and motor function in neurodegeneration by monitoring zebrafish motor activity and sensory responses, using systems such as the zebrabox $(79,80)$. When combined with high-throughput screening of small molecule compounds, such assays can also be powerful in identifying and testing therapeutic options $(62,81)$. Besides that the zebrafish is a suitable genetic model to study the impact of nDNA defects in mitochondrial disease (82), the zebrafish is also a useful model to study the mtDNA, as illustrated in chapter 7 , where we studied the mtDNA bottleneck in mtDNA segregation and transmission.

\section{- Mice}

Among the animal models described, mice are closest to humans in terms of their development, genetic profile and the physiological processes. However, the main drawback of this model is the labor intensiveness, high costs and significant amount of time needed to create a genetic knock-out or mutant model, making them not very suitable for highthroughput analysis. Nuclear genes have been mutated in mice, but also the mtDNA. Although the manipulation of mtDNA is challenging due to the many mtDNA copies in a single cell, the development of mice with heteroplasmic mtDNA mutations is crucial to understand pathology and segregation of mtDNA mutations in the manifestation of tissue specific symptoms (83). Direct manipulation of the mtDNA has been established in transmitochondrial "mito-mice" models, which were generated via microinjection of intact mitochondria into the cytoplasm of a pronuclear unicellular fertilized ovum (zygote), resulting in transmitochondrial mice that possess heteroplasmic cells, harboring mutant and wild-type mtDNA (84). Yet, direct micro-injection can result in relatively low levels of heteroplasmy. 
A more efficient approach to generate transgenic mice uses immortalized embryonic stem cells (ES cells) that are transfected with mutant mitochondria and subsequently subject to clonal selection after treatment with rhodamine 6G (R6G), which limits the transmission of endogenous ES cell mtDNA (85). Ova are then harvested from a donor mice (between the eight-cell and blastocyst stages) in which the transfected ES cells are either injected directly or become integrated by co-culturing the ES cells with an eight-cell to morula stage ova (84, 86). The ova is then transferred to a recipient mice. With the injection method transgenic offspring is chimeric, as part of the animal its cells derive from the host blastocyst and part of the transfected ES cells (87). By ES co-culturing a founder mice can be obtained that is completely developed from the transfected ES cells (84). Transmitochondrial heteroplasmic mice, carrying a single mtDNA deletion, were generated that revealed respiratory chain deficiency with severe mitochondrial symptoms $(88,89)$. Additionally, heteroplasmic mice have been generated by cytoplasmic fusion off inbred strains, creating mice with variable heteroplasmy levels, but no pathology, being suitable models to study tissue specific the segregation and contribution of mtDNA genotypes (90). A different approach to manipulate the mtDNA is by disrupting nDNA encoded genes that are responsible for mtDNA replication and maintenance. Especially, DNA polymerase gamma (POLG) and the DNA helicase Twinkle have been targets to indirectly induce an accumulation of mtDNA mutations (91-94). Also in mice, CRISPR/Cas9 has been the latest development to create knockdown and mutant models, where even multiple genes can be edited at the same time (95).

\section{From genetic cause to treatment of mitochondrial disease}

Despite the current successes in resolving the genetic causes of mitochondrial disorders, the translation into effective treatment strategies is lagging behind. In part, this is due the enormous genetic and phenotypic heterogeneity in mitochondrial disease, which makes the prevalence of individuals with similar disorder low and therefore makes it difficult to collect large groups of patients to conduct clinical trials with sufficient statistical power. Also in a research setting, the performance of in vitro compound screenings is complicated due to the limited availability of patient cell lines with a similar genetic defect or with the same biochemical presentation. Besides, in case of mtDNA mutations, variations in heteroplasmy level of a specific mtDNA mutation can cause large differences in clinical presentation, which influences the study of a treatment strategy. Finally, it is more challenging to deliver potential therapeutics into the mitochondria than to the cytoplasm, as several membranes need to be passed to reach the matrix, sometimes requiring implementation of specific mitochondrial targeting peptide sequences (96). 
Currently, only few randomized double-blinded placebo-controlled trials have been completed and there are no curative treatment options available for mitochondrial disorders (97). In industrial settings, the development of novel drugs involves large scale screening of novel and existing compound libraries (98) to identify those with a potential to improve mitochondrial function. Such compound screenings can be based on a variety of readout systems, involving in vitro testing on patient or genetically modified cell lines, in vivo treatment of animal models, or in silico analysis, where structure based protein modeling can be used to screen large libraries of compounds for their potential to interact or bind with specific target proteins. Yet, the development of a successful treatment strategy might also evolve from clinical practice, where treatment of individual patients with different drugs, drug cocktails or dietary therapies might reveal an effective treatment strategy, as illustrated by the palmitic acid responsive patient in chapter 5 and the use of riboflavin in ACAD9 patients (99). Although large scale trials are not possible, individual patients do seem to benefit from certain treatments. It is the current challenge to make this objective and convert it into an evidence-based personalized-medicine strategy, which shows on the one hand sufficient efficacy to warrant treatment and on the other hand is objectively able to select the patients who are responding to the treatment.

\section{Treatment strategies for mitochondrial disease}

Mitochondrial functioning covers multiple metabolic processes that are crucial for cellular viability, including calcium homeostasis, formation of iron-sulphur clusters, $\beta$-oxidation of fatty acids and OXPHOS. A reduced capacity to produce ATP due to a deficiency in the mitochondrial OXPHOS system is considered the primary cause of mitochondrial disease. Treatment strategies have therefore especially focused on the improvement of mitochondrial respiratory capacity. The majority of the therapies, involving physical exercise, diets and small molecule pharmaceuticals, aim to improve mitochondrial respiratory capacity in a generic manner, by stimulating mitochondrial biogenesis, ETC function or the scavenging of Reactive Oxygen Species (ROS). As shown in Figure 1, the rationale behind the use of therapeutic diets, such as the high-fat and caloric restriction diet, but also exercise therapy is based on the stimulation of mitochondrial biogenesis via the transcriptional co-activator of peroxisome proliferator activated receptor- $\gamma 1$ (PGC1) $\alpha$. PGC-1 $\alpha$ (encoded by the PPARGC1A gene) is a key regulator of mitochondrial biogenesis via different hormone receptors, transcription factors and transcriptional co-activators. PGC1 $\alpha$ interacts with ERR hormone receptors, PPAR nuclear receptors (PPAR- $\gamma$ ) and several transcription factors, including the CAMP response elementbinding protein (CREB), nuclear respiratory factors (NRFs), and $Y Y 1$, together controlling the expression of genes related to OXPHOS, $\beta$-oxidation, TCA, mtDNA nucleotide metabolism 
$(100,101)$. As indicated in Figure 1, PGC-1 $\alpha$ becomes activated upon phosphorylation by AMPK and subsequent deacetylation by SIRT1 $(102,103)$. Both exercise and dietary changes can indirectly influence these enzymes, thereby not only increasing the activity of PGC$1 \alpha$, but also increasing the expression level of PGC-1 $\alpha$, leading to increased mitochondrial biogenesis. This particular signaling pathway has therefore also been an interesting target for pharmacologically based strategies (Table 2). Currently, a number of small molecule pharmaceuticals have been developed which interfere either upstream or downstream of PGC-1 $\alpha$ to increase mitochondrial biogenesis (Figure 1). Although, this can increase the absolute number of accurately functioning mitochondria in patients, this approach will also lead to an increase in the number of dysfunctional mitochondria, which could have toxic effects due to elevated ROS production as well. A second group of pharmaceuticals, which also aims to improve mitochondrial function in a generic manner, is based on targeting the mitochondrial ETC dynamics in order to improve mitochondrial respiratory efficiency (Table 2). This can be achieved with pharmaceuticals that consists of natural or modified ETC complex substrates, cofactors or compounds that alter the ETC redox status. A third group of small molecules are improving ROS scavenging (Table 2). Mitochondria are the main source of ROS production in the cell, which is tightly regulated as ROS have an important function in cellular signaling, but if increased can be highly damaging. ROS production is often significantly increased due to ETC defects (104). An impaired transfer of electrons between the different complexes causes increased leakage of electrons and subsequent formation of oxygen radicals (superoxide anions) (105). ROS scavenging pharmaceutics can reduce the mito-toxic effects of ROS either by scavenging oxygen radicals (pharmaceuticals with antioxidant properties) or by the triggering the cell's natural anti-oxidant defense system. As shown in Table 2, some of these pharmaceuticals possess combined properties. In addition, there a some novel compounds of which the precise mechanism of action is unclear, but seem to have an effect on autophagy and/or mitochondrial translation related pathways. In contrast to generic treatment, tailored treatment strategies specifically target the genetic or metabolic defect in the patient. The latter could involve genetic manipulation or the supplementation of specific enzymes, substrates or nucleotides to restore the patient's metabolic deficiency. Due to the large genetic, clinical and biochemical heterogeneity, it is most likely that a 'one size fits all' therapy will not be possible and that tailored intervention therapies and specific combinations of therapeutics will be needed to effectively target each specific subgroup of mitochondrial disease, eventually enabling personalized medicine. 


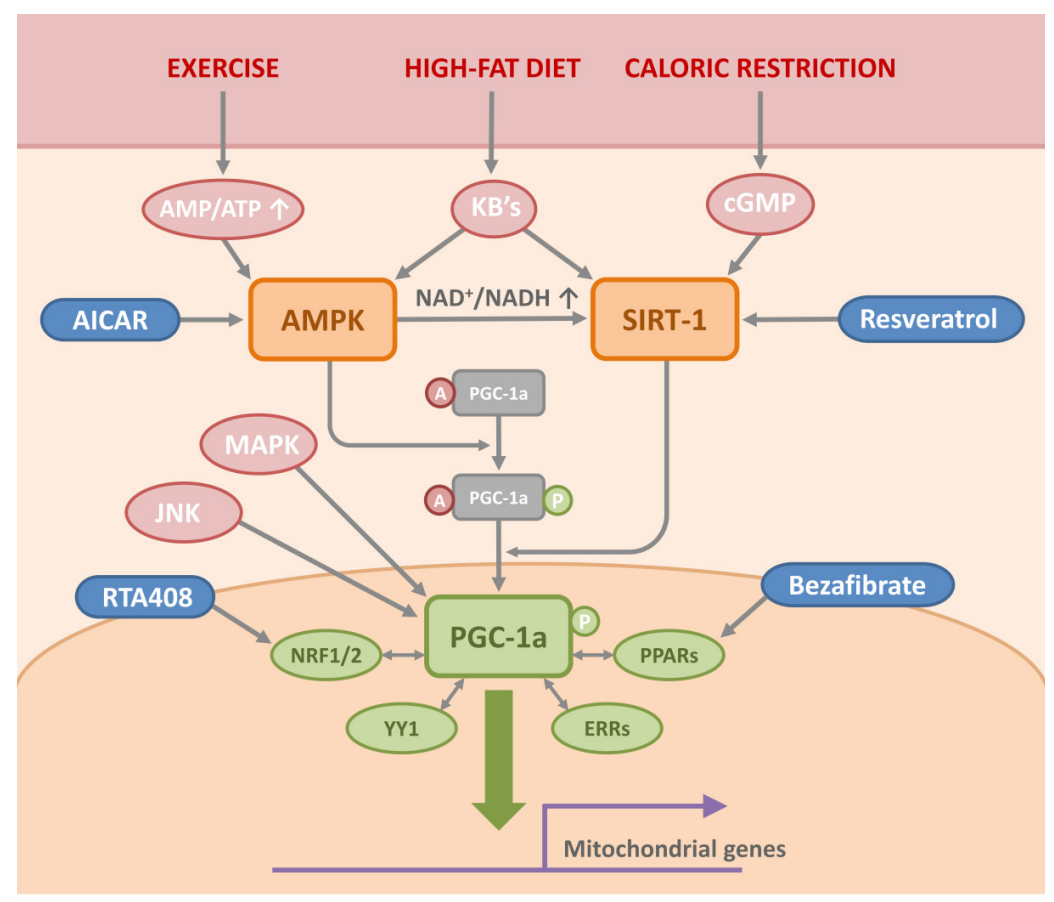

Figure 1: Exercise, diets and small molecule pharmaceuticals stimulate mitochondrial biogenesis as a generic approach to treat mitochondrial disease. The co-transcriptional activator PGC-1 $\alpha$ can be activated directly or indirectly and is a key player in the stimulation of mitochondrial biogenesis via interaction with several transcription factors (PPARs, ERRs, YY1, NRFs). In turn it can induce the expression of a wide range genes involved in mitochondrial functioning.

\section{Generic treatment strategies}

\section{1) Exercise training}

Exercise training aims to enhance mitochondrial function by increasing the number of mitochondria, thereby limiting or compensating the effect of bad functioning mitochondria. Although several studies have indicated a positive effect by improving the OXPHOS function (106), the overall benefits of this therapy are still unclear. Nevertheless, exercise training was shown to be beneficial in terms of mitochondrial function in a randomized trial with ten mitochondrial myopathic patients (107). After a moderate-intensity aerobic training on a treadmill for 8 weeks, the patient's aerobic capacity increased with $30 \%$, blood lactate concentrations at rest and after exercise decreased by $30 \%$, and adenosine diphosphate recovery after exercise improved by more than $60 \%$. However, it has been recommended to perform low-intensity exercise training, as the deconditioning effect could even worsen 
mitochondrial function and therefore have harmful effects. Exercise therapy was initially thought to shift mtDNA heteroplasmy levels and change the ratio of mutant versus wildtype mtDNA molecules in patients with heteroplasmic mtDNA mutations (108). Yet, such therapy in patients with heteroplasmic mtDNA mutations has only shown a mild increase or no change at all in mtDNA heteroplasmy without clear clinical benefits $(109,110)$. Also in relation to resistance training, leading to an increase in muscle strength, no significant changes in mtDNA mutation load could be observed (111). Still, although the ratio does not change, the absolute number of healthy mitochondria increases, which could explain the clinical improvement. The current consensus is that exercise training, including both endurance and resistance exercise, stimulates mitochondrial biogenesis by enhancing the expression levels of PGC-1 $\alpha$ via transcriptional regulation (112-114) and increasing PGC-1 $\alpha$ activation. A single round of exercise already increased the expression of PGC-1 $\alpha$ at mRNA and protein level in skeletal muscle, although PGC-1 $\alpha$ mRNA levels returned to baseline between each round and the increases became smaller with each additional round (115). The latter observations suggest that PGC- $1 \alpha$ gene expression is regulated by transcriptional factors that are sensitive to acute, exercise induced stress. Additionally, exercise training was shown to increase PGC-1 $\alpha$ translocation to the nucleus, and, interestingly also into the mitochondria. Exercise enhanced the interaction between PGC-1 $\alpha$ and the NRF-1 transcription factor in the nucleus, and between PGC-1 $\alpha$ and TFAM at the mitochondrial DNA D-loop (116). As shown in Figure 1, AMPK and SIRT1 are, among others, crucial factors that regulate PGC-1 $\alpha$ activity via, respectively, the phosphorylation and deacetylation of specific PGC-1 $\alpha$ residues. Acute exercise was shown to activate AMPK via the phosphorylation of the Thr172 residue, resulting in increased AMPK activity in skeletal muscle, and longterm exercise even led to an increase in AMPK protein content (117-119). It was proposed that exercise activates AMPK via events that increase the AMP/ATP ratio (Figure1) (120). As AMPK regulates PGC-1 $\alpha$ activity, directly by phosphorylating PGC-1 $\alpha$ and indirectly by promoting the transcription of the PGC-1 $\alpha$ gene, it is another key factor in the pathway of exercise-induced mitochondrial biogenesis $(121,122)$. Mitochondrial gene expression is thus controlled by both transcriptional and post-transcriptional regulations of PGC- $1 \alpha$ that are triggered by the alterations in metabolic demand during exercise. As the effectiveness of exercise therapies is still limited and mainly supportive, combination therapy with dietary or pharmacological supplements seems preferable.

\section{2) Dietary therapies}

High-fat diets were reported to have clinical benefits in some mitochondrial patients and are clinically applied as a supportive treatment option. Most of these reportedly effective 
treatments lacked biochemical prove $(123,124)$ and the mechanisms of action behind these high-fat therapies remained unclear. For proper validation, the diets still need to be tested in a randomized clinical trial. High-fat diets are based on an increased intake of fat and can be applied in combination with a low-carbohydrate diet, which is known as the ketogenicdiet. High-fat diets result in an increase in plasma fatty acids, either in combination with a deprivation of carbohydrates, and are expected to cause a shift from a glycolytic energy metabolism to the beta-oxidation of fatty acids, which is accompanied by the production of ketone bodies in the liver mitochondria via ketogenesis. The ketogenic diet has been beneficial in patients with pyruvate dehydrogenase complex deficiency related brain pathology, where the frequency of epileptic seizures significantly reduced (125). It was suggested that the ketone bodies formed, which are short-chain organic acids (3-betahydroxybutyrate, acetoacetate, and acetone), can freely diffuse across cell membranes and serve as an alternative energy substrate to glucose for the brain and muscles. Ketone bodies are metabolized to acetyl-CoA, which can be used in the citric acid cycle (126). In animal and cellular studies, increased ketone body levels have been related to an increased expression of OXPHOS genes, glutathione levels (127), and mitochondrial biogenesis (128). This phenomenon is also observed during cellular starvation, when factors such AMPK, SIRT1 and PGC-1 $\alpha$ become increasingly activated, stimulating the expression of mitochondrial genes (129). This is fairly similar to the effect of exercise, indicating that, again, an additional general increase of mitochondrial biogenesis can be beneficial. Furthermore, the keto-genic diet was reported once to reduce the mutation load of a heteroplasmic mtDNA deletion in a cybrid model (130). As also illustrated by our patient in chapter 5 , clinical improvements as a result of high-fat intake, with or without carbohydrate restriction, have been reported for complex I deficient patients (131). High fat intake was suggested to bypass the complex I defect as the increased beta-oxidation results in relative more $\mathrm{FADH}_{2}$ (increased FADH $\mathrm{NADH}_{2}$ ratios), which can be a direct substrate for complex II. Other possible mechanism of action of FFAs on mitochondrial function involves the stabilization of OXPHOS complex I and the induction of the PPARY pathway, especially via saturated fatty acids, in this way stimulating mitochondrial biogenesis and proliferation (132-134). Caloric restriction has also been suggested to increase mitochondrial biogenesis by activation of SIRT1 via cGMP, and in turn, activation of the transcriptional coactivator PGC1-alpha (Figure 1). This diet is rarely used in clinical practice, as it is still experimental $(135,136)$.

\section{3) Small molecule pharmaceuticals}

\section{- Pharmaceuticals that increase mitochondrial biogenesis}

As illustrated by the exercise and dietary treatment strategies, increasing the overall mitochondrial mass and total respiratory activity via mitochondrial biogenesis, is considered 
to be beneficial in terms of ATP producing capacity. The mitochondrial biogenesis signaling pathway has therefore been an interesting target for therapeutic manipulation using pharmacological compounds. As shown in Table 2, a number of small molecule pharmaceuticals are under development which aim to activate PGC1 $\alpha$ or its upstream activators. Some of the compounds additionally possess ROS scavenging properties or stimulate the natural anti-oxidant defense system. Increasing the intracellular levels of NAD+ directly, by providing NAD+ precursors (e.g. nicotinamide riboside), or by inhibiting NAD+ consuming enzymes such as the poly(ADP) ribosylpolymerase (PARP inhibitors) stimulated the activity of SIRT1, a NAD+-dependent protein deacetylase, leading to activation of the PGC-1 $\alpha$ pathway $(137,138)$. As shown in Figure 1, Resveratrol is also an activator of SIRT1 (103), increasing expression of PGC-1 $\alpha$, NRF-1 and TFAM (139). The compound has been shown to increase mitochondrial fatty acid oxidation in fibroblasts with CPT2 defects and increased mitochondrial mass and mtDNA content in endothelial cell cultures (140). Another approach is the targeting of the peroxisome proliferator-activated receptors (PPARs) family, which are fatty-acid-regulated nuclear receptors, with three isoforms, $\alpha, \beta / \delta$ and $\gamma$, that interact with its co-activator PGC-1 $\alpha$. Unlike other fibrates, Bezafibrate was shown to have the unique property to activate all three PPAR subtypes as a pan-agonist (141) and also upregulate PGC-1 $\alpha$ gene expression (103). The results in terms of stimulating mitochondrial biogenesis in different mice models (COX10-mKOs, SURF1-Kos, Twinkle helicase "Deletor" mice) are however variable and biogenesis was not induced in all models $(141,142)$. AICAR (5-Aminoimidazole-4-carboxamide ribonucleotide) is an adenosine monophosphate (AMP) analogue (143), able to increase AMPK transcription (144) and phosphorylation of PGC1a (145). AICAR is likely to have combined properties as it was reported to increase both mitochondrial biogenesis and ATP production and in parallel decrease reactive oxygen species in complex I deficient fibroblasts (146). Also, the pharmaceutical compound RTA408 was suggested to have a combined effect, as it increases the activation of the Nrf2 transcription factor by inducing dissociation of Keap1 from Nrf2 (Figure 1), and stimulating the expression of anti-oxidant enzymes (147). As shown in Table 2, the efficacy of most of the compounds that stimulate mitochondrial biogenesis is still being studied in a preclinical stage, or, in ongoing, early phase, clinical trials (some trials were completed without reporting outcomes). Therefore, evidence for the efficacy of these compounds in a clinical setting is still limited.

\section{- $\quad$ Targeting mitochondrial ETC dynamics}

Another strategy to improve the ATP producing capacity is by targeting the mitochondrial ETC dynamics with small molecule pharmaceuticals that improve the respiratory efficiency (Table 2). This could be achieved with ETC complex substrates, cofactors or compounds that alter the ETC redox status. Unlike strategies that are based on stimulation of mitochondrial 
biogenesis, improving the ETC efficiency is likely to be more effective in patients with homoplasmic mtDNA defects or nDNA defects, as there is no fraction of "healthy" mitochondria present that can be increased. However, also for this group of pharmaceuticals, the completion of double-blinded clinical trials is needed to validate their treatment efficacy in mitochondrial disease.

The compound Elamipretide, also known as MTP-131, is an example of how the ATP producing capacity can be increased by modulating the inner mitochondrial membrane phospholipid cardiolipin $(148,149)$. Cardiolipin has a crucial function in cristae formation, mitochondrial fusion, mtDNA stability and segregation. In addition, it contributes to the organization of the respiratory complexes into supercomplexes, and therefore can influence the activity of ETC complexes, especially complex I and complex IV. Studies in dogs with heart failure showed an improved mitochondrial state- 3 respiration and membrane potential, and a reduction in the opening of the MPTP after treatment with Elamipretide. Furthermore, Elamipretide resulted in normalized activity of mitochondrial complex I and IV, increased maximum rate of ATP synthesis and increased ATP/ADP ratio in the left-ventricle (LV) myocardium of the treated dogs (149-151). Elamipretide has entered the clinical trials for treatment of mitochondrial myopathies and LHON. Another example of a compound that increases the bioavailability of OXPHOS substrates, is the compound DCA (dichloroacetate). DCA is a structural analog of pyruvate and is able to lock the pyruvate dehydrogenase complex, which converts pyruvate into acetyl-coA, in an active state via the inhibition of pyruvate dehydrogenase kinase. The resulting increase in acetyl-coA, a substrate for the citric acid cycle, is suggested to improve OXPHOS function $(152,153)$. However, a phase 2 clinical trial on patients with MELAS was ended prematurely because of peripheral nerve toxicity (154). In order to bypass ETC complex I defects, a cell-permeable variant of the complex II substrate succinate was developed under the name NVP015. This substrate has, comparable to succinate, the property to donate electrons directly to complex II and could therefore be beneficial for patients with a mitochondrial complex I deficiency. Also, Coenzyme $Q_{10}$ $\left(\mathrm{CoQ}_{10}\right)$, which is an essential ETC component, has the property to reduce the impact of complex I defects, as it shuttles electrons from complex I, complex II and from the oxidation of fatty acids and branched-chain amino acids (via flavin-linked dehydrogenases) to ETC complex III. Additionally, $\mathrm{CoQ}_{10}$ functions as a lipid-soluble antioxidant that scavenges ROS species. Having a combined effect as a ROS scavenger and ETC component, it is involved in different pathways of cellular metabolism (155). $\mathrm{CoQ}_{10}$ has been evaluated in a phase 2 clinical trial for MELAS, LHON, and CPEO, and revealed minor improvement (156). These compounds are often subject to structural modifications to further improve anti-oxidant capacity, compound uptake or membrane permeability, and among others has led to the development of $\mathrm{CoQ}_{10}$ variants such as Idebenone, which has improved blood-brain barrier 
permeability, and EPI-743, which has increased anti-oxidant capacity (157). Idebenone was developed as a synthetic analog of $\mathrm{CoQ}_{10}$, and has comparable properties in terms of antioxidant and electron carrying function (158-160). Idebenone is available on the market in Europe for the treatment of LHON. EPI-743 has a combined effect as well by improving the ETC dynamics as a cofactor of the NAD(P)H dehydrogenase Quinone 1 (NQO1) and by increasing the expression of the anti-oxidant glutathione (GSH) (161). EPI-743 is currently evaluated in several clinical phase 2 studies (LS, LHON, and PS) $(157,162,163)$. Cyclosporin $A$ is a widely used immunosuppressant that influences mitochondrial dynamics by inhibiting the mitochondrial permeability transition pore (PTP). Cyclosporin A improved mitochondrial respiratory function in cardiomyocytes (164) and induced muscle regeneration, improved mitochondrial function, and reduced muscle apoptosis in patients with collagen VIdeficiency related myopathy (165). Additionally, some novel strategies that are based on redox-agents, such as phenothiazines (e.g. Methylene blue), are being investigated. These agents aim to improve the biochemical activity of specific mitochondrial ETC components by modifying the redox status (166). Although, many of these compounds have entered the clinical trials for particular subgroups of mitochondrial disease, most of these studies are ongoing or the outcomes of completed studies have not (yet) been reported, therefore evidence for efficacy remains to be seen (Table 2).

\section{- Anti-oxidant therapy}

ROS can be a consequence of defects in the ETC chain, but can also further damage ETC complexes and membrane lipids. It affects mitochondrial quality in a vicious circle. Studies on primary fibroblasts from patients with isolated complex I deficiency indicated that that affected cells were depolarized, have increased levels of NADH, affected mitochondrial morphology, and elevated levels of ROS (146, 167-170). Increased ROS levels were also observed in fibroblasts carrying mutations in other OXPHOS complexes and assembly factors (171-174) and in cells treated with OXPHOS inhibitors (175-178), suggesting that an increased level of ROS is a typical event in primary mitochondrial dysfunction $(179,180)$. Moreover, a correlation was shown between NADH and ROS levels and the expression of fully assembled, mature complex I. ROS levels increased with a decrease in the expression of active complex I (169). ROS is therefore considered a major source and enhancer of mitochondrial dysfunction, which is strengthened by the observation that some of the severely affected mitochondrial patients have a relatively mild decrease in ETC complex activity with no drastic reduction in ATP production. In the natural mitochondrial anti-oxidant system, glutathione is, in combination with superoxide dismutase (SOD), glutathione peroidase (GPx) and glutathione reductase (GR), a crucial component in the scavenging of free oxygen radicals, avoiding damage to the ETC complexes and the peroxidation of mitochondrial lipids. Cardiolipin is a phospholipid that is important for the formation of the OXPHOS supercomplexes in the 
mitochondrial inner membrane and influences mitochondrial respiration efficiency (181). Decreased levels of GSH or excessive ROS production causes peroxidation of mitochondrial lipids, such as cardiolipin (182), and can therefore affect the formation of mitochondrial supercomplexes.

ROS production has been an important target in development of mitochondrial treatment strategies, where a wide variety of natural (e.g. vitamins) and pharmaceutical antioxidants has been investigated with the aim to reduce intracellular radical formation (Table 2 ). Anti-oxidant compounds can have ROS scavenging properties themselves or stimulate the natural anti-oxidant defense system (e.g GSH production). Other compounds, like CoQ10 and its derivatives, have a combined effect on mitochondrial function as a ROS scavenger and ETC component (as described above), and is often prescribed as anti-oxidant to treat mitochondrial disease (157). On the other hand, compounds like RP103 and RTA408 aim to stimulate the cell's natural anti-oxidant system. RP103 increases the cellular bioavailability of cysteine, an important amino acid for the synthesis of GSH (183). RTA408 activates the Nrf2 transcription factor (Figure 1)(147) which does not only induce mitochondrial biogenesis (184), but is also a regulator of the cellular antioxidant response (185), among others by increasing the expression of cellular antioxidants (186). Other biomolecules that were shown to display anti-oxidant capacity include Lipoic acid (187) and curcumin (188, 189). KH176 was commercially developed as a synthetic anti-oxidant (190).

\section{- Inducing autophagy}

The immunosuppressant rapamycin is an inhibitor of the mTOR kinase, a molecule that suppresses cellular autophagy and stimulates cell growth (191). Interestingly, mTOR was shown to control mitochondrial oxidative function through a YY1-PGC-1alpha transcriptional complex that induces mitochondrial gene expression, where, controversially, the mTOR suppressor rapamycin was found to decrease mitochondrial gene expression and oxygen consumption (192). Rapamycin was shown to delay onset of symptoms in a Leigh syndrome mouse models and to have an inhibitory effect on aging $(193,194)$. However, the mechanism by which mTOR inhibition exerts these effects are unclear. In addition, the immunosuppressive function of rapamycin has many drastic side effects (191). Enhancing effects on autophagy were also reported for treatment with lithium chloride, possibly via the mTOR pathway (195), although the precise mechanisms behind this compound is also unclear $(196,197)$. As shown in Table 2, results of clinical trials using these suppressors of cellular autophagy are scarcely available. 


\section{Conclusion}

In conclusion, the majority of pharmaceutical compounds for treating mitochondrial disease are currently being evaluated in an early phase clinical trial (phase 1/2), or are still in a preclinical phase. Well-designed clinical trials need to be completed in order to prove the, often anecdotic, clinical benefits. For many compounds, the efficacy has only been shown preclinically in vitro or in vivo, mainly at a biochemical level, with contradictory results reported. Not only it is difficult to obtain a sufficient number of patients with a similar genetic defect to complete a clinical trial, but even patients with the same genetic defect might respond differently to a particular pharmaceutical. The heterogeneity in compound responsiveness makes it difficult to identify the specific groups of patients that would benefit from the treatment. This is also evident from the positive and negative results that have been in case reports. 


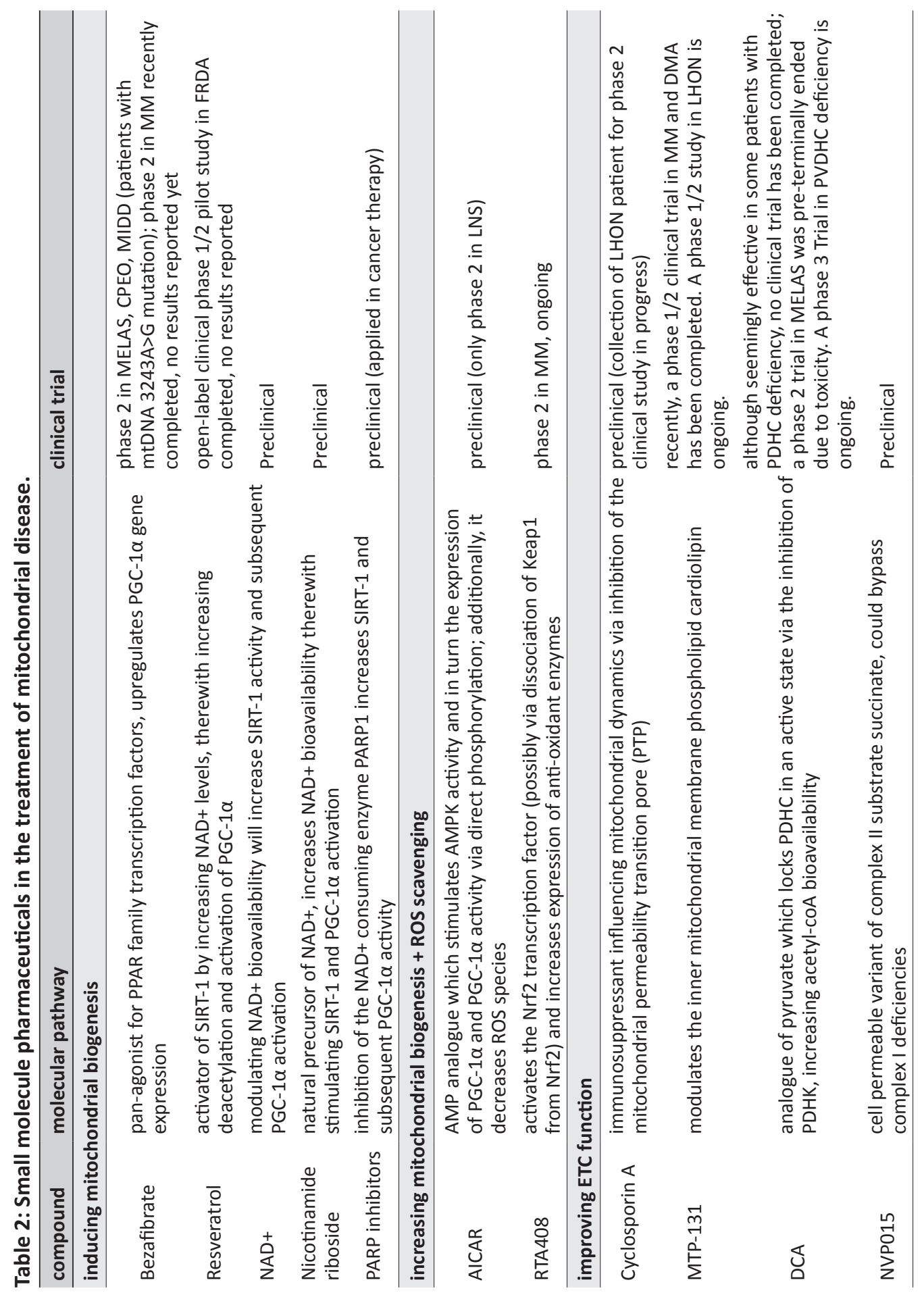




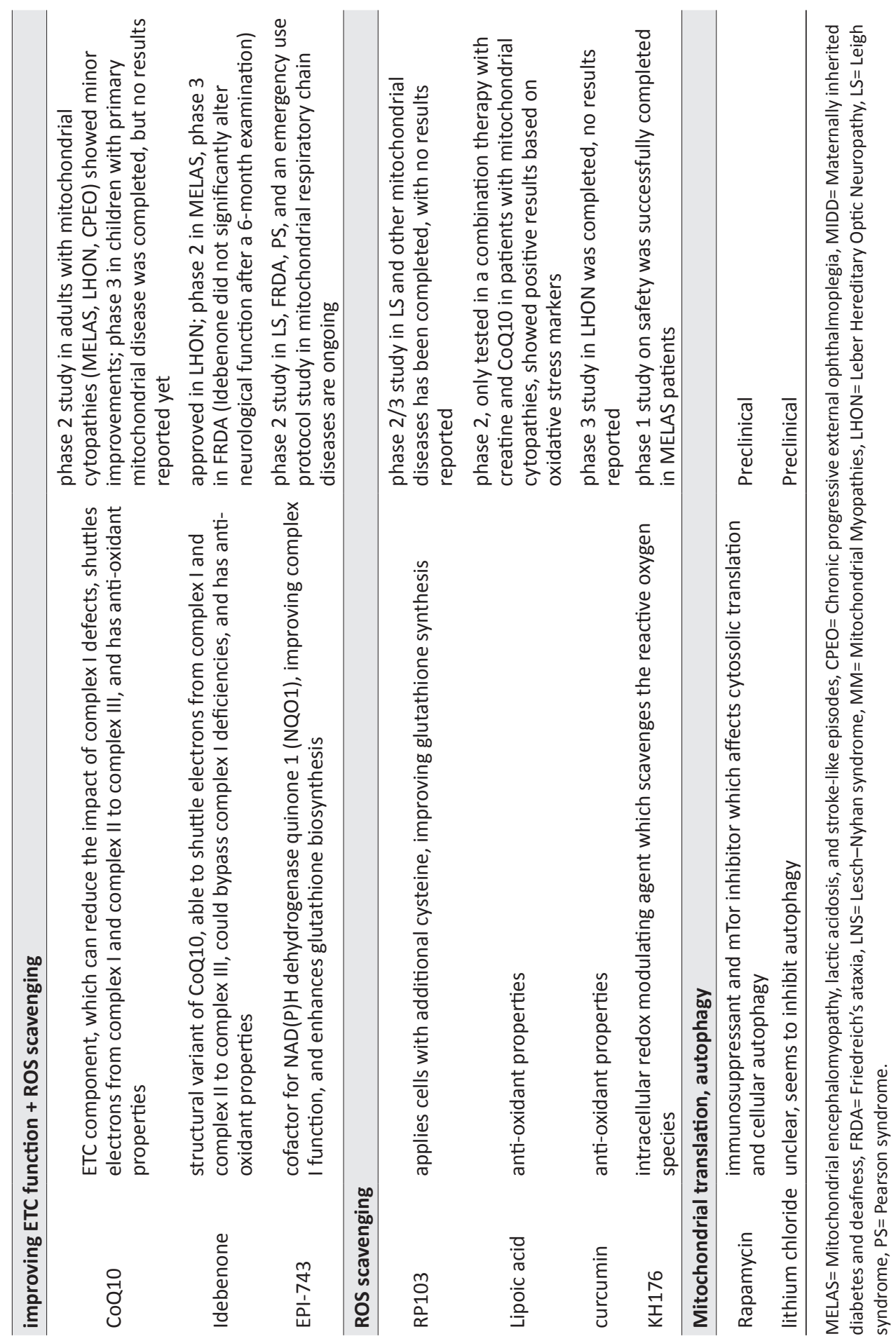




\section{Tailored treatment strategies}

\section{1) Targeting the enzyme or substrate deficiency}

The most effective approach to treat patients with a known genetic defect is to directly target the metabolic deficiency, in which the mutated gene is involved. This approach was successful in Leigh syndrome due to a defect in the SLCA19A3 gene. SLC19A3 is a transmembrane thiamine (vitamin B1) transporter and a defect resulted in a deficiency in intracellular thiamine, which functions as a crucial cofactor in the citric acid cycle. Administration of thiamine to the patients turned out to be highly effective in preventing fatal Leigh syndrome from developing. As a result, neurologists are currently advised to treat patients who are suspected of Leigh syndrome directly with thiamine, even if the genetic cause is unknown. This strategy is based on the fact that SLC19A3 defects are relatively frequent, early treatment can prevent severe and fatal symptoms from occurring, and thiamine does not cause harm in case the genetic cause is different $(198,199)$. Another application of such an approach is demonstrated by a patient from our WES cohort, who manifested with early-onset ataxia, myoclonia, dysarthria, muscle weakness and exercise intolerance due to a defect in SLC25A32, which is a mitochondrial flavin adenine dinucleotide (FAD) transporter. The mutations resulted in a deficiency of FAD, which is a cofactor in the ETC (200). Administration of Riboflavin, the precursor of FAD, led to clinical improvements in this patient (201). Another example of treatment directly compensating the genetic defect are patients with a primary or secondary $\mathrm{CoQ}_{10}$ deficiency, causing encephalomyopathy, severe infantile multi-systemic disease, cerebellar ataxia, isolated myopathy, and nephrotic syndrome, which improved by CoQ10 supplementation (202). Interestingly, dietary therapies can also sometimes be used as a tailored strategy to treat specific inborn errors in metabolism. For example, in patients with a pyruvate dehydrogenase complex (PDHC) deficiency, the resulting lack of acetyl-CoA production causes a substrate deficiency for the citric acid cycle, causing decreased levels of $\mathrm{NADH}$ and $\mathrm{FADH}_{2}$, and in turn ATP production. A ketogenic or high-fat diet was shown to partially compensate for the shortage of acetyl-CoA by increasing the acetyl-CoA production via the beta-oxidation of fatty acids with beneficial effects for patients with PDHC deficiency (203).

For treating mtDNA depletion disorders, due to enzymatic defects in the dNTP salvage pathway or the de novo synthesis pathway, in vitro and preclinical studies have shown positive results for both deoxyribonucleotide (dNMPs, dNTPs) and deoxyribonucleoside (dNs, no phosphate group) supplementation $(204,205)$. This was shown to increase the mtDNA copy number in primary cell cultures from patients with deoxyguanosine kinase (DGUOK) deficiency $(206,207)$ and in a mouse model for MNGIE (207) and thymidine 
kinase 2 (TK2) deficiency. In the TK2 deficient mice, this resulted in improved mitochondrial OXPHOS function, and prolonged life span (208). As also defects in RRM2B, which catalyzes the terminal step of the de novo synthesis of deoxyribonucleosides, cause a mitochondrial depletion syndrome, and could therefore possibly benefit from nucleoside treatment, this has raised controversies in the treatment of a child with a mutation in the RRM $2 B$ gene (Charlie Gard case). Instead of supplementing dNTPs, deoxyribonucleosides, the dNTP building blocks, have been suggested to be more effective in treating these particular mtDNA depletion disorders, as they pass the gut without being degraded, eventually entering the mitochondria to become converted to dNTPs by the intact enzymes (19-07-2017, media communication in Mitochondrial Disease News according to the insights of the patient's physician, Dr. Michio Hirano).

For gene defects which cannot be directly targeted, we are currently developing a pathwaybased strategy. In Figure 2, an example of a patient with an MTFMT mutation is shown. MTFMT is a mitochondrial methionyl-tRNA transformylase, responsible for formylation of methionine and initiating translation of mitochondrial proteins. By evaluating the entire pathway, we characterized multiple targets for interventions, based on available, though partly experimental compounds, on which we developed a treatment strategy. Targeting PDF (enzyme responsible for deformylation of methionine) in patient fibroblasts with antibiotics and compound inhibitors should shift the substrate towards an increased formylated state and rescue mitochondrial translation. Five different compounds were predicted useful to shift this balance, including the PDF inhibiting antibiotic Actinonin, and several MTFMT substrates such as Folinic acid, Formate, Tetrahydrofolic acid, and 5-Methyltetrahydrofolic acid. As COX1 protein levels are significantly reduced in MTFMT deficient fibroblasts due to the translational defect (209), we can use patient fibroblast with different defects in MTFMT as a read-out model to measure the effect of each intervention compound on COX1 protein expression (by ELISA). This shows the theoretical framework of a pathway-based approach, which needs to be experimentally confirmed. Yet, in clinical practise the question is often "has the treatment found its gene?", implying that treatment of individual patients with different drugs, drug cocktails or dietary therapies might reveal an effective treatment strategy, even before knowing the genetic cause. As an example, we demonstrated that riboflavin could be beneficial for patients with an ACAD9 defect and a disturbed complex I assembly. Increasing the levels of the riboflavin cofactor restored complex I activity, possibly by compensating a disturbed ACAD9-FAD binding or by enhancing the assembly of complex I $(99,210)$. Also this is illustrated by the palmitic acid responsive patient in chapter $5(211)$. 


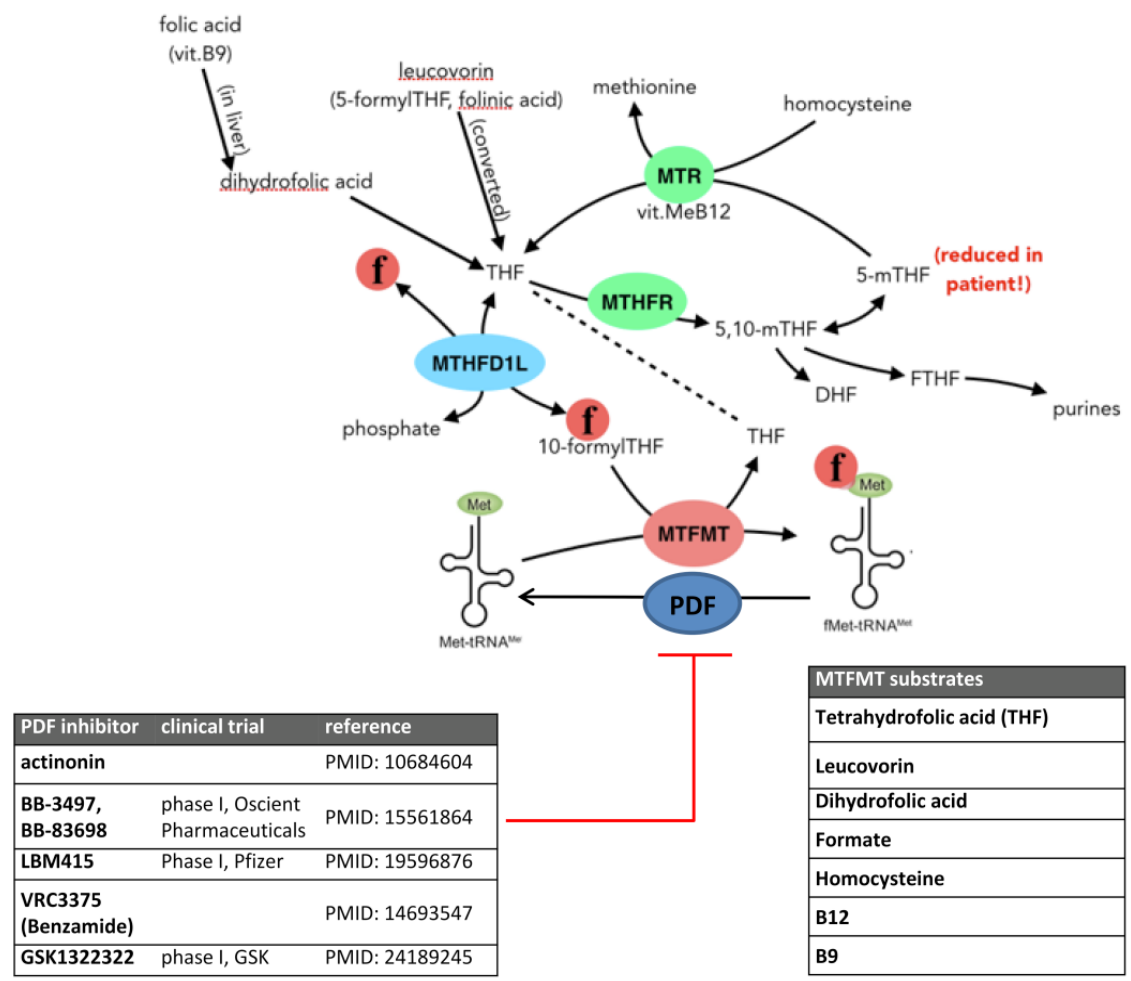

Figure 2: A pathway based approach to treat patients with MTFMT defects. By targeting the PDF enzyme, which is responsible for deformylation of methionine, with antibiotics and compound inhibitors, the substrate shifts towards an increased formylated state and rescue mitochondrial translation. Also the administration of MTFMT substrates might increase the formylated methionine levels.

\section{2) Manipulating mtDNA heteroplasmy levels}

- mtDNA targeted nucleases: ZFN, TALEN, CRISPR/Cas9

A strategy to treat mtDNA disorders at the genetic level involves the use of mitochondria targeted restriction endonucleases, which are able to cleave the mutated mtDNA and shift the mtDNA heteroplasmy levels to wild-type ameliorating the biochemical defect (212-214). This approach was shown successful in cell lines and mice with heteroplasmic mtDNA mutations $(213,215,216)$. An advantage of restriction endonucleases to gene transfer therapies (discussed in more detail below) is that they do not require stable vector integration into the genome with possible mutagenic effects involved, but can be fitted into AAV vectors, infecting host cells without integration into the genome. Yet, removing mutated mtDNA copies will only be helpful in case of heteroplasmic mutations. A technical limitation of restriction site specific endonucleases is that these can only be applied if the mutation introduces a unique restriction site that is not present in wild-type human mtDNA. 
Nevertheless, mutation-specific restriction endonucleases were shown to be effective for the m.8993T>G mutation in NARP (216). It was demonstrated in cells heteroplasmic for the m.8993T>G mutation (78\% heteroplasmy), which causes NARP at heteroplasmy levels $>75 \%$ and Leigh syndrome at heteroplasmy levels $>90 \%$, that the mitochondrial targeted R.Xmal restriction endonuclease selectively eliminated mutant mtDNA. This resulted in cells with significantly increased rates of oxygen consumption and ATP production (217). Interestingly, as NARP only manifests at high heteroplasmy levels (especially the m.8993T>C mutation causing symptoms $>90 \%$ heteroplasmy), a small decrease in mutation load might already be sufficient to reach non-pathogenic heteroplasmy levels, circumventing toxic mtDNA depletion.

The need for a restriction site can in part be overcome by zinc finger nucleases (ZFNs) or Transcription Activator-Like Effector Nucleases (TALENs), which can target unspecific restriction enzymes to specific sites in the mtDNA or nuclear genome via assembly of ZFN or TALE modules $(213,218)$. Zinc finger nucleases consists of tandem repeats of zinc fingers (usually three) that bind the DNA nucleotides in a specific manner, and a Fokl, non-specific endonuclease domain, functioning as the DNA cleavage module (219). A dimer pair of ZFNs, consisting of individual ZFNs that bind opposite DNA strands, is needed for fusion of the cleavage modules, and in turn induce specific DNA cleavage. The composition of dimeric ZFNs ensures that the cleavage enzyme is only active at the targeted site, largely avoiding problems with constitutive, non-specific, nuclease activity (214). The latter was a serious problem of the early developed ZFN systems and could limit their use in a clinical setting $(220,221)$. Mitochondria targeted ZFNs were shown to cause small shifts in heteroplasmy levels by selectively degrading mtDNA molecules with the m.8993T $>$ G point mutation, which causes NARP, and the large and relative frequent 4977bp mtDNA deletion, which causes either chronic progressive external ophthalmoplegia (CPEO), Kearns-Sayre (KS) or Pearson syndrome (214). Further investigations are needed, especially in in vivo models, to overcome the current difficulties in delivering these modules in an appropriate concentration to the affected tissue and validate whether biochemical improvements occur.

TALENs are comparable to ZNFs in that both use DNA binding motifs to target a nonspecific nuclease to cleave DNA at a specific site. TALENs were suggested to have higher potential and more straight-forward design than ZFNs (222). TALENs consist of the TALE elements, repetitive domain modules that bind individual nucleotides, making them target specific (223) in combination with a non-specific nuclease such as Fokl (224). Studies have proven that mitochondrial localized TALENs can specifically target mtDNA copies carrying the m.8483_13459del4977 deletion, and the m.14459G4A point mutation in the MT-ND6 gene in a cybrid cell model (213). TALENs were able to decrease the number of mtDNA 
molecules carrying the large m.8483_13459del4977 deletion, together with stimulating the amplification of wild-type mtDNA copies. This shift in heteroplasmy level was maintained, even after the TALENs were no longer present, indicating a high potential for treatment (213). Interestingly, also in the context of disease transmission, (mito)TALENs were able to reduce the mtDNA mutation load in mice oocytes via the elimination of the mutant mtDNA copies, in specific for mutations that cause LHON and NARP (225). Further studies are required to investigate the possible toxicity that is caused by the rapid reduction in mtDNA copy-number, which is involved in all different approaches that are based on targeted mtDNA degradation. Such rapid reductions in mtDNA copy number are comparable to the mtDNA depletion events seen in certain mitochondrial disorders. It is therefore important to study the role for stimulation of mtDNA biogenesis to bypass this problem. Apart from patient treatment, these approaches could also be applied to reduce the risk of carry-over of mutated mtDNA in mitochondrial replacement therapy. A drawback of both ZNF and TALENs compared to site-specific endonucleases, is that these require larger constructs which cannot always easily fit into AAV vectors.

The latest development based on endonucleases is the CRISPR (clustered, regularly interspaced, short, palindromic repeats)/Cas (CRISPR-associated) system, which is, unlike the ZFNs and TALENs, based on a Cas nuclease (Cas9) that induces double strand breaks. The Cas nuclease is directed to a target DNA sequence using a short CRISPR guide RNA sequence (226). Like ZFN and TALEN, the CRISPR/Cas system can be applied for genome editing, either by introducing random mutations at the site of DNA cleavage by non-homologous end joining, or by introducing specific mutations by co-injection of a template DNA construct with homology to the sequences surrounding the cleavage site. NHEJ-mediated repair of Cas induced double strand breaks is especially useful in generating a null allele, knockout, in the gene of interest, as NHEJ is prone to introducing in-del errors. For treating both nDNA and mtDNA diseases at the genomic level, a DNA template is required to induce homology directed repair (HDR), and specifically introduce the desired base substitution. An advantage of CRISPR/Cas above ZFNs and TALENs is that the guide RNAs can be more easily adapted to a specific DNA site, allowing the targeting of nearly any sequence in the genome with much higher efficiency. Also, distinct guide RNA sequences can be applied the same time to direct the Cas nuclease towards multiple DNA target-sites, modifying the DNA in a multiplexed manner $(226,227)$. CRISPR/Cas9 is more specific than ZFNs and TALENs, which can exhibit context-dependent binding preferences (228-230). CRISPR/Cas9 is currently the most promising strategy for gene editing and has been reported in vitro, in somatic and IPSC cells (231-238) and in vivo $(95,239-243)$. However, the potential of this system in correcting heteroplasmic and homoplasmic mtDNA mutations is currently under investigation. Despite that the CRISPR/Cas system can be targeted into the mitochondria (244), a major obstacle 
in treating mtDNA defects remains the fact that CRISPR/Cas gene-editing will not change all mutated mtDNA copies in the cell and therefore it is still the question whether the shift in heteroplasmy level will be sufficient to reach the tissue specific biochemical threshold needed for normal mitochondrial respiration. In a personal communication at Euromit 2017 by Carlos Moraes (Miami, Florida) it was reported that mitoTALENs are at this moment, still, more effective than CRISPR/Cas9 in manipulating mtDNA heteroplasmy levels.

\section{- mtDNA manipulation with peptide nucleic acids}

Another approach to selectively manipulate mtDNA replication favoring the wild-type mtDNA, is to use peptide nucleic acid (PNA) oligomers. PNA oligomers are synthetic DNA analogues, in which the deoxyribose phosphate backbone has been replaced by aminoethyl glycine units that are connected to the conventional bases (245). Due to a similar spacing between the individual bases in the PNA oligomer as in nucleic acid structures, these oligomers have high affinity to hybridize with complementary DNA sequences. PNA oligomers even have higher binding affinity for nucleic acids than the complementary DNA strands. PNA oligomers are resistant to nucleases and proteases $(246,247)$, making them effective molecules in antisense therapies (248). However, PNAs are barely permeable to the cell membrane $(249,250)$, and therefore require active support to pass the cell's lipid bilayers (251), for example by delivering the PNAs with cationic detergents (252) or conjugation to peptides that catalyse membrane permeation $(252,253)$. As heteroplasmic mtDNA patients carry both mutated and wild-type mtDNA copies, PNAs can be used to selectively bind the mutated mtDNA molecules, in this way counteracting mtDNA replication. The net result is a preferred amplification of the wild-type mtDNA and a shift in heteroplasmy levels (249). PNAs could also be designed to interfere with translation of the mutated gene. Although, PNA oligomers can be delivered to the cytosol, targeting them into the mitochondria remains a major challenge (254).

\section{3) Gene editing of nDNA mutations: ZFN, TALEN, CRISPR/Cas9}

In nDNA editing, approaches based on ZFNs, TALENs or CRISPR/CAS are dependent on a delivery system (e.g. via injection or AAV vectors), cell type and the cell's natural repair mechanism. Whereas non-homologues end-joining, following specific cleavage, can generate gene knockout models or remove a protein that carries a dominant negative mutation, triggering of the homology directed recombination (HDR) machinery by a template DNA strand introduces or removes a specific mutation. These approaches can in principle edit all homozygous, compound heterozygous and dominant heterozygous nDNA defects, allowing the treatment of both recessive and dominant disorders $(255,256)$. All three methods have their limitations and complications in terms of off-target effects and 
the generation of mosaics (Table 3). As reported above, the more recent developed CRISPR/ Cas9 system was shown to have several advantages in nDNA editing compared to ZFNs and TALENs (257). Still, also when using CRISPR/Cas9 to treat homozygous mutations, the undamaged copy of the mutated allele could be used as a repair template instead of the supplied DNA fragment, resulting in less efficient genome editing and a mosaic situation. CRSIPR/Cas9-mediated mosaicism may also occurs when nucleases do not just cut the DNA at the one cell stage of embryonic development, or when there is prolonged expression and activity of Cas9 in the embryo (258-260). In addition, HDR can result in different mutations at the restricted site despite the presence of a DNA template, and therefore does not always result in the desired base substitution. Nevertheless, several reports have demonstrated the ability of CRISPR-Cas9 in in vivo gene editing using adeno-associated viruses (AAVs) vectors, of which the safety and efficacy have been tested in clinical trials (261) and could potentially be clinically applied $(256,262-265)$. In neuromuscular disease, the intramuscular, intraperitoneal or intravenous injection of CRISPR-Cas9 components corrected the recessive $X$-linked gene-defect (Dmd) in mouse models of Duchenne muscular dystrophy, and rescued the disease phenotype $(266,267)$. Although, it was shown that CRISPR/Cas9 can edit the nDNA in vitro and in vivo, CRISPR/Cas9 mediated gene editing is still premature and needs further development to achieve high efficiency without harmful effects.

Table 3: the advantages and disadvantages of ZFN, TALEN and CRISPR/Cas9 in genome editing.

\begin{tabular}{cccccc}
\hline Nuclease & Engineering & Efficiencies & Specificity & Toxicity & Clinical development \\
\hline ZFN & difficult & high & low & medium & phase $1 / 2$ \\
TALEN & easy & medium & medium & medium & phase 1 \\
Cas9/sgRNA & very easy & high & high & low & preclinical \\
\hline
\end{tabular}

\section{4) Gene transfer therapy for nDNA and mtDNA disease}

Gene therapy is based on the introduction of a wild-type gene into patient cells in order to obtain a therapeutic benefit. Although, most studies on gene therapy have been preclinical, it is a promising field in the treatment of mitochondrial disease, especially the eye-diseases. Independent of the gene transfer method and type of therapy, this strategy requires cloning of a "healthy" gene copy into a vector vehicle. The vector is used to deliver the therapeutic gene to the target cells of the patient, and allow integration of the genetic material into the host nDNA or mtDNA. By introducing a "healthy" gene into an individual's sperm-cells, egg -cells or embryonic stem cells, germline gene therapy could prevent disease transmission towards progeny. However, in contrast to somatic gene therapy, gene transfer into germ cells is currently forbidden in most countries for ethical reasons. Somatic gene therapy introduces genes into the somatic cells of an individual. It was shown 
successful in treating nDNA encoded gene defects in vitro and in vivo (268-270). Different viral and non-viral gene delivery systems have been developed, which all have their own benefits, limitations and side-effects and which are all highly dependent on the targeted tissue type, either in vitro or in vivo (271). Non-viral systems are based on physical (naked DNA, DNA bombardant, electroporation, hydrodynamic, ultrasound, magnetofection) or chemical (cationic lipids, different cationic polymers, lipid polymers) techniques to transfer the transgene into the host's cell nucleus. Different viral vectors can be used (retroviral, adenoviral, adeno association, helper-dependent adenoviral systems, hybrid adenoviral systems, herpes simplex, pox virus, lentivirus, Epstein-Barr virus) to transduce the gene of interest into the host's genome (271). The transfection of host cells is more efficient with viral methods than with non-viral methods, although a drawback of applying viral vectors is the immunogenicity, cytotoxicity and safety (272). Random integration of the transgene into the host nDNA can cause insertional mutagenesis, which could lead to a disrupted expression of tumor suppressor genes or activation of oncogenes. Adeno-associated vectors (AAV) vectors were developed to have a replication deficiency and are safer than adenoviral vectors. Interestingly, they have the ability to integrate into a specific site on chromosome 19 (although, AAV vector genomes can also persist within cells as episomes without integration), reducing the chances of insertional mutagenesis.

AAVs have been used in the treatment of several diseases (273-276). Nevertheless, targeting human skeletal muscle or passing the blood-brain barrier remains very difficult task in treating nDNA encoded mitochondrial disorders. The potential for gene transfer therapy in treating nDNA encoded mitochondrial defects was among others demonstrated by AAVmediated hepatic ETHE1 gene expression in a murine model for ethylmalonic encephalopathy, caused by ETHE1 mutations. ETHE1 gene therapy restored sulphur dioxygenase activity and restored biochemical abnormalities in liver, muscle, and brain, resulting in an increased life span (277). Also in treating mitochondrial neurogastrointestinal encephalopathy (MNGIE), which is caused by mutations in the thymidine phosphorylase gene TYMP, delivering of a human TYMP coding sequence (hcTYMP) under the control of a liver-specific promoter prevented biochemical imbalance in a murine model of MNGIE (278). Other reports that demonstrated the success of AAV based gene transfer included a study on a Harlequin mouse model with mitochondrial complex 1 deficiency which could be recovered by the intra-vitreal administration of an AAV2/2_AlF1 vector (279) and the phenotypic rescue of a MPV17 knockout (KO) mice by AAV vector expression of human MPV17 (280).

Modifying the mtDNA and mitochondria is a major challenge as each mitochondrion contains many copies of mtDNA and the mitochondrial membrane is difficult to cross. An alternative strategy to partially overcome these difficulties is based on "allotopic" expression 
of mtDNA encoded proteins. Genes that are mtDNA localized and translated by the mitoribosomes are then delivered and integrated into the host nDNA, by altering the genetic code and using a viral or non-viral vector. To allow the expressed proteins to import into the mitochondria, the transgene coding sequences requires the addition of a mitochondrial targeting peptide sequence at the $\mathrm{N}$-terminus (281). Several studies have focused on the allotropic expression of complex I subunits in order to treat the most common mtDNA disorder LHON, which most commonly results from mutations in the ND1, ND4 and ND6 genes $(282,283)$. It was demonstrated that expression of synthetic ND4 increased ATP synthesis in a human cells harboring the m.11778G4A ND4 mutation (282). In murine LHON models, the intraocular injection of human ND4 gene expressed by AAV vectors was shown to prevent retinal ganglion cells loss and improve optic atrophy $(264,283)$. Nevertheless, it is a question if allotopically-expressed proteins, if imported, always properly integrate within the respiratory chain (284).

\section{5) Stem cell approaches}

There is increasing support that the use of stem cell therapies could improve mitochondrial disease, especially in relation to neuromuscular and neurodegenerative symptoms (285, 286). Different types of stem cells can be used to treat or prevent disease. As for nDNA gene editing or gene therapy, stem cell therapies are not a specific approach for mitochondrial disorders, but some interesting results have been obtained in a number of mitochondrial studies. Exogenous stem cell therapy for mitochondrial disease caused by nDNA mutations was shown beneficial for patients with MNGIE, a mtDNA depletion syndrome, that causes severe gastrointestinal malabsorption, encephalopathy, leukodystrophy, and myopathy (287-290). Hematopoetic and bone marrow stem cell transplantation could reduce the high, and toxic levels of circulating thymidine that are characteristic in MNGIE, by introducing cells with normal thymidine phosphorylase activity (287, 290-293). Endogenous stem cell therapy is a novel field in the treatment of mitochondrial disease, and is, in our lab, being investigated, as a potential treatment for myopathy due to mtDNA mutations. Myogenic stem cells, called mesoangioblasts, in the muscle of patients with the m.3243A $>\mathrm{G}$ mutations, generally harbor few or no mutated mtDNA copies, in contrast to post-mitotic muscle tissue. Culturing and delivering these stem cells through the blood stream to the patient muscle tissue is expected to increase muscle mass and the amount of wild-type mtDNA (294). Although, further studies are needed to prove the clinical benefits, this method has the potential to decrease the mtDNA load and improve biochemistry in mature patient muscle (295). 


\section{Conclusion}

WES and WGS have significantly contributed to the characterization and understanding of the genetic basis of mitochondrial disease, and has led to the development of a myriad of functional in vitro and in vivo models to study the underlying pathophysiological processes. Our knowledge of the mechanisms underlying mitochondrial disorders has tremendously improved. Still, despite some successes for gene-specific treatment in specific patients, there is still a long way to go on the road to therapy. Both, generic and tailored treatment studies show promising results, but most often in restricted subgroups of mitochondrial disease or even individual patients. It is therefore likely that therapeutic strategies will become highly personalized, where combinations of generic treatment approaches and tailored strategies might come together to establish patient specific treatments for mitochondrial disease. 


\section{References}

1. Jacobs LJ, de Wert G, Geraedts JP, de Coo IF, Smeets HJ. The transmission of OXPHOS disease and methods to prevent this. Hum Reprod Update. 2006;12(2):119-36.

2. Sallevelt $S$, de Koning B, Szklarczyk R, Paulussen ADC, de Die-Smulders CEM, Smeets HJM. A comprehensive strategy for exome-based preconception carrier screening. Genet Med. 2017;19(5):583-92.

3. Carroll CJ, Brilhante V, Suomalainen A. Next-generation sequencing for mitochondrial disorders. British journal of pharmacology. 2014;171(8):1837-53.

4. Yu Y, Wu BL, Wu J, Shen Y. Exome and whole-genome sequencing as clinical tests: a transformative practice in molecular diagnostics. Clinical chemistry. 2012;58(11):1507-9.

5. Gonzaga-Jauregui C, Lupski JR, Gibbs RA. Human genome sequencing in health and disease. Annual review of medicine. 2012;63:35-61.

6. Belkadi A, Bolze A, Itan Y, Cobat A, Vincent QB, Antipenko A, et al. Whole-genome sequencing is more powerful than whole-exome sequencing for detecting exome variants. Proc Natl Acad Sci U S A. 2015;112(17):5473-8.

7. Kremer LS, Bader DM, Mertes C, Kopajtich R, Pichler G, luso A, et al. Genetic diagnosis of Mendelian disorders via RNA sequencing. Nature communications. 2017;8:15824.

8. Wrighton KH. Genetic testing: The diagnostic power of RNA-seq. Nature reviews Genetics. 2017;18(7):392-3.

9. Lloyd RE, McGeehan JE. Structural analysis of mitochondrial mutations reveals a role for bigenomic protein interactions in human disease. PLoS One. 2013;8(7):e69003.

10. McCafferty CL, Sergeev YV. In silico Mapping of Protein Unfolding Mutations for Inherited Disease. Scientific reports. 2016;6:37298.

11. de Carvalho MD, De Mesquita JF. Structural modeling and in silico analysis of human superoxide dismutase 2. PLoS One. 2013;8(6):e65558.

12. Brown D. Systems biology-What's in a name? American journal of physiology Cell physiology. 2008;294(6):C1299.

13. Balaban RS. Modeling mitochondrial function. American journal of physiology Cell physiology. 2006;291(6):C1107-13.

14. Jafri MS, Kumar R. Modeling mitochondrial function and its role in disease. Progress in molecular biology and translational science. 2014;123:103-25.

15. Koopman WJ, Beyrath J, Fung CW, Koene S, Rodenburg RJ, Willems PH, et al. Mitochondrial disorders in children: toward development of small-molecule treatment strategies. EMBO molecular medicine. 2016;8(4):311-27.

16. Diodato D, Melchionda L, Haack TB, Dallabona C, Baruffini E, Donnini C, et al. VARS2 and TARS2 mutations in patients with mitochondrial encephalomyopathies. Hum Mutat. 2014;35(8):983-9.

17. Mitra K, Lippincott-Schwartz J. Analysis of mitochondrial dynamics and functions using imaging approaches. Current protocols in cell biology. 2010;Chapter 4:Unit 425 1-1.

18. Brand MD, Nicholls DG. Assessing mitochondrial dysfunction in cells. The Biochemical journal. 2011;435(2):297-312.

19. Diaz F, Barrientos A, Fontanesi F. Evaluation of the mitochondrial respiratory chain and oxidative phosphorylation system using blue native gel electrophoresis. Curr Protoc Hum Genet. 2009; Chapter 19:Unit19 4.

20. Ebert AD, Liang P, Wu JC. Induced pluripotent stem cells as a disease modeling and drug screening platform. Journal of cardiovascular pharmacology. 2012;60(4):408-16.

21. Zhao $Y$, Yin $X$, Qin $H$, Zhu F, Liu H, Yang W, et al. Two supporting factors greatly improve the efficiency of human iPSC generation. Cell stem cell. 2008;3(5):475-9. 
22. Prigione A. Induced pluripotent stem cells (iPSCs) for modeling mitochondrial DNA disorders. Methods Mol Biol. 2015;1265:349-56.

23. Yap MS, Nathan KR, Yeo Y, Lim LW, Poh CL, Richards M, et al. Neural Differentiation of Human Pluripotent Stem Cells for Nontherapeutic Applications: Toxicology, Pharmacology, and In Vitro Disease Modeling. Stem cells international. 2015;2015:105172.

24. Prigione $A$, Lichtner $B$, Kuhl $H$, Struys EA, Wamelink $M$, Lehrach $H$, et al. Human induced pluripotent stem cells harbor homoplasmic and heteroplasmic mitochondrial DNA mutations while maintaining human embryonic stem cell-like metabolic reprogramming. Stem cells (Dayton, Ohio). 2011;29(9):1338-48.

25. Hamalainen RH, Manninen T, Koivumaki H, Kislin M, Otonkoski T, Suomalainen A. Tissue- and cell-type-specific manifestations of heteroplasmic mtDNA 3243A $>\mathrm{G}$ mutation in human induced pluripotent stem cell-derived disease model. Proc Natl Acad Sci U S A. 2013;110(38):E3622-30.

26. Pickrell AM, Youle RJ. Mitochondrial disease: mtDNA and protein segregation mysteries in iPSCs. Current biology : CB. 2013;23(23):R1052-4.

27. Pye D, Kyriakouli DS, Taylor GA, Johnson R, Elstner M, Meunier B, et al. Production of transmitochondrial cybrids containing naturally occurring pathogenic mtDNA variants. Nucleic Acids Res. 2006;34(13):e95.

28. Wilkins HM, Carl SM, Swerdlow RH. Cytoplasmic hybrid (cybrid) cell lines as a practical model for mitochondriopathies. Redox biology. 2014;2:619-31.

29. Vithayathil SA, Ma Y, Kaipparettu BA. Transmitochondrial cybrids: tools for functional studies of mutant mitochondria. Methods Mol Biol. 2012;837:219-30.

30. Lasserre JP, Dautant A, Aiyar RS, Kucharczyk R, Glatigny A, Tribouillard-Tanvier D, et al. Yeast as a system for modeling mitochondrial disease mechanisms and discovering therapies. Disease models \& mechanisms. 2015;8(6):509-26.

31. Mileykovskaya E, Penczek PA, Fang J, Mallampalli VK, Sparagna GC, Dowhan W. Arrangement of the respiratory chain complexes in Saccharomyces cerevisiae supercomplex III2IV2 revealed by single particle cryo-electron microscopy. The Journal of biological chemistry. 2012;287(27):23095-103.

32. Bonnefoy N, Fox TD. Genetic transformation of Saccharomyces cerevisiae mitochondria. Methods in cell biology. 2001;65:381-96.

33. Okamoto K, Perlman PS, Butow RA. The sorting of mitochondrial DNA and mitochondrial proteins in zygotes: preferential transmission of mitochondrial DNA to the medial bud. The Journal of cell biology. 1998;142(3):613-23.

34. Meunier B. Site-directed mutations in the mitochondrially encoded subunits I and III of yeast cytochrome oxidase. The Biochemical journal. 2001;354(Pt 2):407-12.

35. Meunier B, Fisher N, Ransac S, Mazat JP, Brasseur G. Respiratory complex III dysfunction in humans and the use of yeast as a model organism to study mitochondrial myopathy and associated diseases. Biochim Biophys Acta. 2013;1827(11-12):1346-61.

36. Feuermann M, Francisci S, Rinaldi T, De Luca C, Rohou H, Frontali L, et al. The yeast counterparts of human 'MELAS' mutations cause mitochondrial dysfunction that can be rescued by overexpression of the mitochondrial translation factor EF-Tu. EMBO reports. 2003;4(1):53-8.

37. Montanari A, Besagni C, De Luca C, Morea V, Oliva R, Tramontano A, et al. Yeast as a model of human mitochondrial tRNA base substitutions: investigation of the molecular basis of respiratory defects. RNA (New York, NY). 2008;14(2):275-83.

38. Bourgeron $T$, Rustin $P$, Chretien D, Birch-Machin M, Bourgeois $M$, Viegas-Pequignot $E$, et al. Mutation of a nuclear succinate dehydrogenase gene results in mitochondrial respiratory chain deficiency. Nat Genet. 1995;11(2):144-9.

39. Calvo SE, Mootha VK. The mitochondrial proteome and human disease. Annu Rev Genomics Hum Genet. 2010;11:25-44. 
40. Koopman WJ, Willems PH, Smeitink JA. Monogenic mitochondrial disorders. N Engl J Med. 2012;366(12):1132-41.

41. Vafai SB, Mootha VK. Mitochondrial disorders as windows into an ancient organelle. Nature. 2012;491(7424):374-83.

42. Mashkevich G, Repetto B, Glerum DM, Jin C, Tzagoloff A. SHY1, the yeast homolog of the mammalian SURF-1 gene, encodes a mitochondrial protein required for respiration. The Journal of biological chemistry. 1997;272(22):14356-64.

43. Bestwick $M$, Jeong MY, Khalimonchuk $\mathrm{O}$, Kim H, Winge DR. Analysis of Leigh syndrome mutations in the yeast SURF1 homolog reveals a new member of the cytochrome oxidase assembly factor family. Mol Cell Biol. 2010;30(18):4480-91.

44. Foriel S, Willems P, Smeitink J, Schenck A, Beyrath J. Mitochondrial diseases: Drosophila melanogaster as a model to evaluate potential therapeutics. The international journal of biochemistry \& cell biology. 2015;63:60-5.

45. Guo M. Drosophila as a model to study mitochondrial dysfunction in Parkinson's disease. Cold Spring Harbor perspectives in medicine. 2012;2(11).

46. Bier E. Drosophila, the golden bug, emerges as a tool for human genetics. Nature reviews Genetics. 2005;6(1):9-23.

47. Rubin GM, Yandell MD, Wortman JR, Gabor Miklos GL, Nelson CR, Hariharan IK, et al. Comparative genomics of the eukaryotes. Science (New York, NY). 2000;287(5461):2204-15.

48. Hay BA, Huh JR, Guo M. The genetics of cell death: approaches, insights and opportunities in Drosophila. Nature reviews Genetics. 2004;5(12):911-22.

49. Hay BA, Guo M. Caspase-dependent cell death in Drosophila. Annual review of cell and developmental biology. 2006;22:623-50.

50. Bellen HJ, Tong C, Tsuda H. 100 years of Drosophila research and its impact on vertebrate neuroscience: a history lesson for the future. Nature reviews Neuroscience. 2010;11(7):514-22.

51. Adams MD, Sekelsky JJ. From sequence to phenotype: reverse genetics in Drosophila melanogaster. Nature reviews Genetics. 2002;3(3):189-98.

52. Ren $X$, Sun J, Housden BE, Hu Y, Roesel C, Lin S, et al. Optimized gene editing technology for Drosophila melanogaster using germ line-specific Cas9. Proc Natl Acad Sci U S A. 2013;110(47):19012-7.

53. Gospodaryov DV, Lushchak OV, Rovenko BM, Perkhulyn NV, Gerards M, Tuomela T, et al. Ciona intestinalis NADH dehydrogenase NDX confers stress-resistance and extended lifespan on Drosophila. Biochim Biophys Acta. 2014;1837(11):1861-9.

54. Haley B, Hendrix D, Trang V, Levine M. A simplified miRNA-based gene silencing method for Drosophila melanogaster. Developmental biology. 2008;321(2):482-90.

55. Elbashir SM, Martinez J, Patkaniowska A, Lendeckel W, Tuschl T. Functional anatomy of siRNAs for mediating efficient RNAi in Drosophila melanogaster embryo lysate. EMBO J. 2001;20(23):687788.

56. Sanchez-Martinez A, Luo N, Clemente P, Adan C, Hernandez-Sierra R, Ochoa P, et al. Modeling human mitochondrial diseases in flies. Biochim Biophys Acta. 2006;1757(9-10):1190-8.

57. Lessing $D$, Bonini NM. Maintaining the brain: insight into human neurodegeneration from Drosophila melanogaster mutants. Nature reviews Genetics. 2009;10(6):359-70.

58. Lu B, Vogel H. Drosophila models of neurodegenerative diseases. Annual review of pathology. 2009;4:315-42.

59. Xu H, DeLuca SZ, O'Farrell PH. Manipulating the metazoan mitochondrial genome with targeted restriction enzymes. Science (New York, NY). 2008;321(5888):575-7.

60. Burman JL, Itsara LS, Kayser EB, Suthammarak W, Wang AM, Kaeberlein M, et al. A Drosophila model of mitochondrial disease caused by a complex I mutation that uncouples proton pumping from electron transfer. Disease models \& mechanisms. 2014;7(10):1165-74. 
61. Pandey UB, Nichols CD. Human disease models in Drosophila melanogaster and the role of the fly in therapeutic drug discovery. Pharmacological reviews. 2011;63(2):411-36.

62. Steele SL, Prykhozhij SV, Berman JN. Zebrafish as a model system for mitochondrial biology and diseases. Translational research : the journal of laboratory and clinical medicine. 2014;163(2):7998.

63. Otten AB, Theunissen TE, Derhaag JG, Lambrichs EH, Boesten IB, Winandy M, et al. Differences in Strength and Timing of the mtDNA Bottleneck between Zebrafish Germline and Non-germline Cells. Cell Rep. 2016;16(3):622-30.

64. Artuso L, Romano A, Verri T, Domenichini A, Argenton F, Santorelli FM, et al. Mitochondrial DNA metabolism in early development of zebrafish (Danio rerio). Biochim Biophys Acta. 2012;1817(7):1002-11.

65. Howe K, Clark MD, Torroja CF, Torrance J, Berthelot C, Muffato M, et al. The zebrafish reference genome sequence and its relationship to the human genome. Nature. 2013;496(7446):498-503.

66. Broughton RE, Milam JE, Roe BA. The complete sequence of the zebrafish (Danio rerio) mitochondrial genome and evolutionary patterns in vertebrate mitochondrial DNA. Genome Res. 2001;11(11):1958-67.

67. Doyon Y, McCammon JM, Miller JC, Faraji F, Ngo C, Katibah GE, et al. Heritable targeted gene disruption in zebrafish using designed zinc-finger nucleases. Nature biotechnology. 2008;26(6):702-8.

68. Huang P, Xiao A, Zhou M, Zhu Z, Lin S, Zhang B. Heritable gene targeting in zebrafish using customized TALENs. Nature biotechnology. 2011;29(8):699-700.

69. Hwang WY, Fu Y, Reyon D, Maeder ML, Tsai SQ, Sander JD, et al. Efficient genome editing in zebrafish using a CRISPR-Cas system. Nature biotechnology. 2013;31(3):227-9.

70. Woods IG, Kelly PD, Chu F, Ngo-Hazelett P, Yan YL, Huang H, et al. A comparative map of the zebrafish genome. Genome Res. 2000;10(12):1903-14.

71. Postlethwait JH, Woods IG, Ngo-Hazelett P, Yan YL, Kelly PD, Chu F, et al. Zebrafish comparative genomics and the origins of vertebrate chromosomes. Genome Res. 2000;10(12):1890-902.

72. Goldsmith JR, Jobin C. Think small: zebrafish as a model system of human pathology. Journal of biomedicine \& biotechnology. 2012;2012:817341.

73. Nasevicius A, Ekker SC. Effective targeted gene 'knockdown' in zebrafish. Nat Genet. 2000;26(2):216-20.

74. Yuan S, Sun Z. Microinjection of mRNA and morpholino antisense oligonucleotides in zebrafish embryos. Journal of visualized experiments : JoVE. 2009(27).

75. Hogg RC, Bandelier F, Benoit A, Dosch R, Bertrand D. An automated system for intracellular and intranuclear injection. Journal of neuroscience methods. 2008;169(1):65-75.

76. Wang W, Liu X, Gelinas D, Ciruna B, Sun Y. A fully automated robotic system for microinjection of zebrafish embryos. PLoS One. 2007;2(9):e862.

77. Spaink HP, Cui C, Wiweger MI, Jansen HJ, Veneman WJ, Marin-Juez R, et al. Robotic injection of zebrafish embryos for high-throughput screening in disease models. Methods. 2013;62(3):24654.

78. Suster ML, Kikuta H, Urasaki A, Asakawa K, Kawakami K. Transgenesis in zebrafish with the tol2 transposon system. Methods Mol Biol. 2009;561:41-63.

79. Giacomotto J, Rinkwitz S, Becker TS. Effective heritable gene knockdown in zebrafish using synthetic microRNAs. Nature communications. 2015;6:7378.

80. Gibbs EM, Horstick EJ, Dowling JJ. Swimming into prominence: the zebrafish as a valuable tool for studying human myopathies and muscular dystrophies. Febs J. 2013;280(17):4187-97.

81. MacRae CA, Peterson RT. Zebrafish as tools for drug discovery. Nature reviews Drug discovery. 2015;14(10):721-31. 
82. D'Agati G, Beltre R, Sessa A, Burger A, Zhou Y, Mosimann C, et al. A defect in the mitochondrial protein Mpv17 underlies the transparent casper zebrafish. Developmental biology. 2017;430(1):11-7.

83. Tyynismaa $\mathrm{H}$, Suomalainen A. Mouse models of mitochondrial DNA defects and their relevance for human disease. EMBO reports. 2009;10(2):137-43.

84. Pinkert CA, Trounce IA. Production of transmitochondrial mice. Methods. 2002;26(4):348-57.

85. Sligh JE, Levy SE, Waymire KG, Allard P, Dillehay DL, Nusinowitz $\mathrm{S}$, et al. Maternal germ-line transmission of mutant mtDNAs from embryonic stem cell-derived chimeric mice. Proc Natl Acad Sci U S A. 2000;97(26):14461-6.

86. Kasahara A, Ishikawa K, Yamaoka M, Ito M, Watanabe N, Akimoto M, et al. Generation of transmitochondrial mice carrying homoplasmic mtDNAs with a missense mutation in a structural gene using ES cells. Hum Mol Genet. 2006;15(6):871-81.

87. Pinkert CA, Irwin MH, Johnson LW, Moffatt RJ. Mitochondria transfer into mouse ova by microinjection. Transgenic research. 1997;6(6):379-83.

88. Inoue K, Nakada K, Hayashi J, Isobe K. [Generation of mice with mitochondrial dysfunction by introducing mouse mtDNA carrying a deletion into zygotes]. Tanpakushitsu kakusan koso Protein, nucleic acid, enzyme. 2001;46(7):829-37.

89. Nakada K, Sato A, Sone H, Kasahara A, Ikeda K, Kagawa Y, et al. Accumulation of pathogenic DeltamtDNA induced deafness but not diabetic phenotypes in mito-mice. Biochem Biophys Res Commun. 2004;323(1):175-84.

90. Jenuth JP, Peterson AC, Fu K, Shoubridge EA. Random genetic drift in the female germline explains the rapid segregation of mammalian mitochondrial DNA. Nat Genet. 1996;14(2):14651.

91. Kaguni LS. DNA polymerase gamma, the mitochondrial replicase. Annual review of biochemistry. 2004;73:293-320.

92. Graziewicz MA, Longley MJ, Copeland WC. DNA polymerase gamma in mitochondrial DNA replication and repair. Chemical reviews. 2006;106(2):383-405.

93. Milenkovic D, Matic S, Kuhl I, Ruzzenente B, Freyer C, Jemt E, et al. TWINKLE is an essential mitochondrial helicase required for synthesis of nascent D-loop strands and complete mtDNA replication. Hum Mol Genet. 2013;22(10):1983-93.

94. Tyynismaa H, Mjosund KP, Wanrooij S, Lappalainen I, Ylikallio E, Jalanko A, et al. Mutant mitochondrial helicase Twinkle causes multiple mtDNA deletions and a late-onset mitochondrial disease in mice. Proc Natl Acad Sci U S A. 2005;102(49):17687-92.

95. Yang $\mathrm{H}$, Wang $\mathrm{H}$, Jaenisch $\mathrm{R}$. Generating genetically modified mice using CRISPR/Cas-mediated genome engineering. Nat Protoc. 2014;9(8):1956-68.

96. Yamada $\mathrm{Y}$, Akita $\mathrm{H}$, Kogure $\mathrm{K}$, Kamiya $\mathrm{H}$, Harashima $\mathrm{H}$. Mitochondrial drug delivery and mitochondrial disease therapy--an approach to liposome-based delivery targeted to mitochondria. Mitochondrion. 2007;7(1-2):63-71.

97. Chinnery P, Majamaa K, Turnbull D, Thorburn D. Treatment for mitochondrial disorders. The Cochrane database of systematic reviews. 2006(1):CD004426.

98. Sahdeo S, Tomilov A, Komachi K, Iwahashi C, Datta S, Hughes O, et al. High-throughput screening of FDA-approved drugs using oxygen biosensor plates reveals secondary mitofunctional effects. Mitochondrion. 2014;17:116-25.

99. Gerards M, van den Bosch BJ, Danhauser K, Serre V, van Weeghel M, Wanders RJ, et al. Riboflavinresponsive oxidative phosphorylation complex I deficiency caused by defective ACAD9: new function for an old gene. Brain. 2011;134(Pt 1):210-9.

100. Andreux PA, Houtkooper RH, Auwerx J. Pharmacological approaches to restore mitochondrial function. Nature reviews Drug discovery. 2013;12(6):465-83. 
101. Dominy JE, Puigserver P. Mitochondrial biogenesis through activation of nuclear signaling proteins. Cold Spring Harbor perspectives in biology. 2013;5(7).

102. Canto C, Auwerx J. PGC-1alpha, SIRT1 and AMPK, an energy sensing network that controls energy expenditure. Current opinion in lipidology. 2009;20(2):98-105.

103. Kanabus M, Heales SJ, Rahman S. Development of pharmacological strategies for mitochondrial disorders. British journal of pharmacology. 2014;171(8):1798-817.

104. Murphy MP, Holmgren A, Larsson NG, Halliwell B, Chang CJ, Kalyanaraman B, et al. Unraveling the biological roles of reactive oxygen species. Cell Metab. 2011;13(4):361-6.

105. Zorov DB, Juhaszova M, Yaniv Y, Nuss HB, Wang S, Sollott SJ. Regulation and pharmacology of the mitochondrial permeability transition pore. Cardiovascular research. 2009;83(2):213-25.

106. Jeppesen TD, Schwartz M, Olsen DB, Wibrand F, Krag T, Duno M, et al. Aerobic training is safe and improves exercise capacity in patients with mitochondrial myopathy. Brain. 2006;129(Pt 12):3402-12.

107. Taivassalo T, De Stefano N, Argov Z, Matthews PM, Chen J, Genge A, et al. Effects of aerobic training in patients with mitochondrial myopathies. Neurology. 1998;50(4):1055-60.

108. Taivassalo T, De Stefano N, Chen J, Karpati G, Arnold DL, Argov Z. Short-term aerobic training response in chronic myopathies. Muscle Nerve. 1999;22(9):1239-43.

109. Taivassalo T, Shoubridge EA, Chen J, Kennaway NG, DiMauro S, Arnold DL, et al. Aerobic conditioning in patients with mitochondrial myopathies: physiological, biochemical, and genetic effects. Ann Neurol. 2001;50(2):133-41.

110. Taivassalo T, Gardner JL, Taylor RW, Schaefer AM, Newman J, Barron MJ, et al. Endurance training and detraining in mitochondrial myopathies due to single large-scale mtDNA deletions. Brain. 2006;129(Pt 12):3391-401.

111. Murphy JL, Blakely EL, Schaefer AM, He L, Wyrick P, Haller RG, et al. Resistance training in patients with single, large-scale deletions of mitochondrial DNA. Brain. 2008;131(Pt 11):283240.

112. Baar K, Wende AR, Jones TE, Marison M, Nolte LA, Chen M, et al. Adaptations of skeletal muscle to exercise: rapid increase in the transcriptional coactivator PGC-1. Faseb J. 2002;16(14):187986.

113. Deldicque L, Atherton P, Patel R, Theisen D, Nielens $H$, Rennie MJ, et al. Effects of resistance exercise with and without creatine supplementation on gene expression and cell signaling in human skeletal muscle. Journal of applied physiology (Bethesda, Md : 1985). 2008;104(2):3718.

114. Garcia-Roves PM, Huss J, Holloszy JO. Role of calcineurin in exercise-induced mitochondrial biogenesis. American journal of physiology Endocrinology and metabolism. 2006;290(6):E11729.

115. Perry CG, Lally J, Holloway GP, Heigenhauser GJ, Bonen A, Spriet LL. Repeated transient mRNA bursts precede increases in transcriptional and mitochondrial proteins during training in human skeletal muscle. The Journal of physiology. 2010;588(Pt 23):4795-810.

116. Little JP, Safdar A, Benton CR, Wright DC. Skeletal muscle and beyond: the role of exercise as a mediator of systemic mitochondrial biogenesis. Applied physiology, nutrition, and metabolism = Physiologie appliquee, nutrition et metabolisme. 2011;36(5):598-607.

117. Leick L, Wojtaszewski JF, Johansen ST, Kiilerich K, Comes G, Hellsten Y, et al. PGC-1alpha is not mandatory for exercise- and training-induced adaptive gene responses in mouse skeletal muscle. American journal of physiology Endocrinology and metabolism. 2008;294(2):E463-74.

118. Clark SA, Chen ZP, Murphy KT, Aughey RJ, McKenna MJ, Kemp BE, et al. Intensified exercise training does not alter AMPK signaling in human skeletal muscle. American journal of physiology Endocrinology and metabolism. 2004;286(5):E737-43. 
119. Sriwijitkamol A, Ivy JL, Christ-Roberts C, DeFronzo RA, Mandarino LJ, Musi N. LKB1-AMPK signaling in muscle from obese insulin-resistant Zucker rats and effects of training. American journal of physiology Endocrinology and metabolism. 2006;290(5):E925-32.

120. Richter EA, Ruderman NB. AMPK and the biochemistry of exercise: implications for human health and disease. The Biochemical journal. 2009;418(2):261-75.

121. Irrcher I, Ljubicic V, Kirwan AF, Hood DA. AMP-activated protein kinase-regulated activation of the PGC-1alpha promoter in skeletal muscle cells. PLoS One. 2008;3(10):e3614.

122. Irrcher I, Ljubicic V, Hood DA. Interactions between ROS and AMP kinase activity in the regulation of PGC-1alpha transcription in skeletal muscle cells. American journal of physiology Cell physiology. 2009;296(1):C116-23.

123. Malojcic B, Brinar V, Poser C, Djakovic V. An adult case of Leigh disease. Clinical neurology and neurosurgery. 2004;106(3):237-40.

124. Laugel V, This-Bernd V, Cormier-Daire V, Speeg-Schatz C, de Saint-Martin A, Fischbach M. Earlyonset ophthalmoplegia in Leigh-like syndrome due to NDUFV1 mutations. Pediatr Neurol. 2007;36(1):54-7.

125. Rogovik AL, Goldman RD. Ketogenic diet for treatment of epilepsy. Canadian family physician Medecin de famille canadien. 2010;56(6):540-2.

126. Klepper J, Diefenbach S, Kohlschutter A, Voit T. Effects of the ketogenic diet in the glucose transporter 1 deficiency syndrome. Prostaglandins, leukotrienes, and essential fatty acids. 2004;70(3):321-7.

127. Jarrett SG, Milder JB, Liang $L P$, Patel $M$. The ketogenic diet increases mitochondrial glutathione levels. J Neurochem. 2008;106(3):1044-51.

128. Bough KJ, Wetherington J, Hassel B, Pare JF, Gawryluk JW, Greene JG, et al. Mitochondrial biogenesis in the anticonvulsant mechanism of the ketogenic diet. Ann Neurol. 2006;60(2):22335.

129. Nunnari J, Suomalainen A. Mitochondria: in sickness and in health. Cell. 2012;148(6):1145-59.

130. Santra S, Gilkerson RW, Davidson M, Schon EA. Ketogenic treatment reduces deleted mitochondrial DNAs in cultured human cells. Ann Neurol. 2004;56(5):662-9.

131. Roef MJ, de Meer K, Reijngoud DJ, Straver HW, de Barse M, Kalhan SC, et al. Triacylglycerol infusion improves exercise endurance in patients with mitochondrial myopathy due to complex I deficiency. Am J Clin Nutr. 2002;75(2):237-44.

132. Hughes SD, Kanabus M, Anderson G, Hargreaves IP, Rutherford T, O'Donnell $M$, et al. The ketogenic diet component decanoic acid increases mitochondrial citrate synthase and complex I activity in neuronal cells. J Neurochem. 2014;129(3):426-33.

133. Kanabus M, Fassone E, Hughes SD, Bilooei SF, Rutherford T, Donnell MO, et al. The pleiotropic effects of decanoic acid treatment on mitochondrial function in fibroblasts from patients with complex I deficient Leigh syndrome. J Inherit Metab Dis. 2016;39(3):415-26.

134. Miglio G, Rosa AC, Rattazzi L, Collino M, Lombardi G, Fantozzi R. PPARgamma stimulation promotes mitochondrial biogenesis and prevents glucose deprivation-induced neuronal cell loss. Neurochemistry international. 2009;55(7):496-504.

135. Hancock CR, Han DH, Higashida K, Kim SH, Holloszy JO. Does calorie restriction induce mitochondrial biogenesis? A reevaluation. Faseb J. 2011;25(2):785-91.

136. Canto C, Auwerx J. Caloric restriction, SIRT1 and longevity. Trends in endocrinology and metabolism: TEM. 2009;20(7):325-31.

137. Bai P, Canto C, Oudart H, Brunyanszki A, Cen Y, Thomas C, et al. PARP-1 inhibition increases mitochondrial metabolism through SIRT1 activation. Cell Metab. 2011;13(4):461-8.

138. Cerutti R, Pirinen E, Lamperti C, Marchet S, Sauve AA, Li W, et al. NAD(+)-dependent activation of Sirt1 corrects the phenotype in a mouse model of mitochondrial disease. Cell Metab. 2014;19(6):1042-9. 
139. Csiszar A, Labinskyy N, Pinto JT, Ballabh P, Zhang H, Losonczy G, et al. Resveratrol induces mitochondrial biogenesis in endothelial cells. Am J Physiol Heart Circ Physiol. 2009;297(1):H1320.

140. Aires V, Delmas D, Le Bachelier C, Latruffe N, Schlemmer D, Benoist JF, et al. Stilbenes and resveratrol metabolites improve mitochondrial fatty acid oxidation defects in human fibroblasts. Orphanet J Rare Dis. 2014;9:79.

141. Yatsuga S, Suomalainen A. Effect of bezafibrate treatment on late-onset mitochondrial myopathy in mice. Hum Mol Genet. 2012;21(3):526-35.

142. Viscomi C, Bottani E, Civiletto G, Cerutti R, Moggio M, Fagiolari G, et al. In vivo correction of COX deficiency by activation of the AMPK/PGC-1alpha axis. Cell Metab. 2011;14(1):80-90.

143. Merrill GF, Kurth EJ, Hardie DG, Winder WW. AICA riboside increases AMP-activated protein kinase, fatty acid oxidation, and glucose uptake in rat muscle. Am J Physiol. 1997;273(6 Pt 1):E1107-12.

144. Terada S, Goto M, Kato M, Kawanaka K, Shimokawa T, Tabata I. Effects of low-intensity prolonged exercise on PGC-1 mRNA expression in rat epitrochlearis muscle. Biochem Biophys Res Commun. 2002;296(2):350-4.

145. Jager S, Handschin C, St-Pierre J, Spiegelman BM. AMP-activated protein kinase (AMPK) action in skeletal muscle via direct phosphorylation of PGC-1alpha. Proc Natl Acad Sci U S A. 2007;104(29):12017-22.

146. Golubitzky A, Dan P, Weissman S, Link G, Wikstrom JD, Saada A. Screening for active small molecules in mitochondrial complex I deficient patient's fibroblasts, reveals AICAR as the most beneficial compound. PLoS One. 2011;6(10):e26883.

147. Liu X, Ward K, Xavier C, Jann J, Clark AF, Pang IH, et al. The novel triterpenoid RTA 408 protects human retinal pigment epithelial cells against $\mathrm{H} 2 \mathrm{O} 2$-induced cell injury via NF-E2-related factor 2 (Nrf2) activation. Redox biology. 2016;8:98-109.

148. Alam NM, Mills WCt, Wong AA, Douglas RM, Szeto HH, Prusky GT. A mitochondrial therapeutic reverses visual decline in mouse models of diabetes. Disease models \& mechanisms. 2015;8(7):701-10.

149. Sabbah HN. Targeting mitochondrial dysfunction in the treatment of heart failure. Expert review of cardiovascular therapy. 2016;14(12):1305-13.

150. Szeto HH. First-in-class cardiolipin-protective compound as a therapeutic agent to restore mitochondrial bioenergetics. British journal of pharmacology. 2014;171(8):2029-50.

151. Wu J, Hao S, Sun XR, Zhang H, Li H, Zhao H, et al. Elamipretide (SS-31) Ameliorates IsofluraneInduced Long-Term Impairments of Mitochondrial Morphogenesis and Cognition in Developing Rats. Frontiers in cellular neuroscience. 2017;11:119.

152. Stacpoole PW, Gilbert LR, Neiberger RE, Carney PR, Valenstein E, Theriaque DW, et al. Evaluation of long-term treatment of children with congenital lactic acidosis with dichloroacetate. Pediatrics. 2008;121(5):e1223-8.

153. Stacpoole PW. The pyruvate dehydrogenase complex as a therapeutic target for age-related diseases. Aging cell. 2012;11(3):371-7.

154. Kaufmann P, Engelstad K, Wei Y, Jhung S, Sano MC, Shungu DC, et al. Dichloroacetate causes toxic neuropathy in MELAS: a randomized, controlled clinical trial. Neurology. 2006;66(3):32430.

155. Turunen M, Olsson J, Dallner G. Metabolism and function of coenzyme Q. Biochim Biophys Acta. 2004;1660(1-2):171-99.

156. Glover El, Martin J, Maher A, Thornhill RE, Moran GR, Tarnopolsky MA. A randomized trial of coenzyme Q10 in mitochondrial disorders. Muscle Nerve. 2010;42(5):739-48.

157. Enns GM, Kinsman SL, Perlman SL, Spicer KM, Abdenur JE, Cohen BH, et al. Initial experience in the treatment of inherited mitochondrial disease with EPI-743. Mol Genet Metab. 2012;105(1):91102. 
158. Giorgio V, Petronilli V, Ghelli A, Carelli V, Rugolo M, Lenaz G, et al. The effects of idebenone on mitochondrial bioenergetics. Biochim Biophys Acta. 2012;1817(2):363-9.

159. Haefeli RH, Erb M, Gemperli AC, Robay D, Courdier Fruh I, Anklin C, et al. NQO1-dependent redox cycling of idebenone: effects on cellular redox potential and energy levels. PLoS One. 2011;6(3):e17963.

160. Jaber S, Polster BM. Idebenone and neuroprotection: antioxidant, pro-oxidant, or electron carrier? Journal of bioenergetics and biomembranes. 2015;47(1-2):111-8.

161. Pastore A, Petrillo S, Tozzi G, Carrozzo R, Martinelli D, Dionisi-Vici C, et al. Glutathione: a redox signature in monitoring EPI-743 therapy in children with mitochondrial encephalomyopathies. Mol Genet Metab. 2013;109(2):208-14.

162. Martinelli D, Catteruccia M, Piemonte F, Pastore A, Tozzi G, Dionisi-Vici C, et al. EPI-743 reverses the progression of the pediatric mitochondrial disease--genetically defined Leigh Syndrome. Mol Genet Metab. 2012;107(3):383-8.

163. Sadun AA, Chicani CF, Ross-Cisneros FN, Barboni P, Thoolen M, Shrader WD, et al. Effect of EPI-743 on the clinical course of the mitochondrial disease Leber hereditary optic neuropathy. Archives of neurology. 2012;69(3):331-8.

164. Sharov VG, Todor A, Khanal S, Imai M, Sabbah HN. Cyclosporine A attenuates mitochondrial permeability transition and improves mitochondrial respiratory function in cardiomyocytes isolated from dogs with heart failure. Journal of molecular and cellular cardiology. 2007;42(1):150-8.

165. Merlini L, Angelin A, Tiepolo T, Braghetta P, Sabatelli P, Zamparelli A, et al. Cyclosporin A corrects mitochondrial dysfunction and muscle apoptosis in patients with collagen VI myopathies. Proc Natl Acad Sci U S A. 2008;105(13):5225-9.

166. Xiong ZM, Choi JY, Wang K, Zhang H, Tariq Z, Wu D, et al. Methylene blue alleviates nuclear and mitochondrial abnormalities in progeria. Aging cell. 2016;15(2):279-90.

167. Koopman WJ, Verkaart S, Visch HJ, van Emst-de Vries S, Nijtmans LG, Smeitink JA, et al. Human NADH:ubiquinone oxidoreductase deficiency: radical changes in mitochondrial morphology? American journal of physiology Cell physiology. 2007;293(1):C22-9.

168. Koopman WJ, Verkaart S, Visch HJ, van der Westhuizen FH, Murphy MP, van den Heuvel LW, et al. Inhibition of complex I of the electron transport chain causes O2-. -mediated mitochondrial outgrowth. American journal of physiology Cell physiology. 2005;288(6):C1440-50.

169. Verkaart S, Koopman WJ, van Emst-de Vries SE, Nijtmans LG, van den Heuvel LW, Smeitink JA, et al. Superoxide production is inversely related to complex I activity in inherited complex I deficiency. Biochim Biophys Acta. 2007;1772(3):373-81.

170. Valsecchi F, Koopman WJ, Manjeri GR, Rodenburg RJ, Smeitink JA, Willems PH. Complex I disorders: causes, mechanisms, and development of treatment strategies at the cellular level. Developmental disabilities research reviews. 2010;16(2):175-82.

171. Lopez-Fabuel I, Le Douce J, Logan A, James AM, Bonvento G, Murphy MP, et al. Complex I assembly into supercomplexes determines differential mitochondrial ROS production in neurons and astrocytes. Proc Natl Acad Sci U S A. 2016;113(46):13063-8.

172. Nouws J, Nijtmans LG, Smeitink JA, Vogel RO. Assembly factors as a new class of disease genes for mitochondrial complex I deficiency: cause, pathology and treatment options. Brain. 2012;135(Pt 1):12-22.

173. Miwa S, Jow H, Baty K, Johnson A, Czapiewski R, Saretzki G, et al. Low abundance of the matrix arm of complex I in mitochondria predicts longevity in mice. Nature communications. 2014;5:3837.

174. Giachin G, Bouverot R, Acajjaoui S, Pantalone S, Soler-Lopez M. Dynamics of Human Mitochondrial Complex I Assembly: Implications for Neurodegenerative Diseases. Frontiers in molecular biosciences. 2016;3:43. 
175. Forkink M, Manjeri GR, Liemburg-Apers DC, Nibbeling E, Blanchard M, Wojtala A, et al. Mitochondrial hyperpolarization during chronic complex I inhibition is sustained by low activity of complex II, III, IV and V. Biochim Biophys Acta. 2014;1837(8):1247-56.

176. Forkink M, Basit F, Teixeira J, Swarts HG, Koopman WJ, Willems PH. Complex I and complex III inhibition specifically increase cytosolic hydrogen peroxide levels without inducing oxidative stress in HEK293 cells. Redox biology. 2015;6:607-16.

177. Distelmaier F, Valsecchi F, Liemburg-Apers DC, Lebiedzinska M, Rodenburg RJ, Heil S, et al. Mitochondrial dysfunction in primary human fibroblasts triggers an adaptive cell survival program that requires AMPK-alpha. Biochim Biophys Acta. 2015;1852(3):529-40.

178. Schockel L, Glasauer A, Basit F, Bitschar K, Truong H, Erdmann G, et al. Targeting mitochondrial complex I using BAY 87-2243 reduces melanoma tumor growth. Cancer \& metabolism. 2015;3:11.

179. Voets AM, Huigsloot M, Lindsey PJ, Leenders AM, Koopman WJ, Willems PH, et al. Transcriptional changes in OXPHOS complex I deficiency are related to anti-oxidant pathways and could explain the disturbed calcium homeostasis. Biochim Biophys Acta. 2012;1822(7):1161-8.

180. Voets AM, Lindsey PJ, Vanherle SJ, Timmer ED, Esseling JJ, Koopman WJ, et al. Patient-derived fibroblasts indicate oxidative stress status and may justify antioxidant therapy in OXPHOS disorders. Biochim Biophys Acta. 2012;1817(11):1971-8.

181. Schagger H. Respiratory chain supercomplexes of mitochondria and bacteria. Biochim Biophys Acta. 2002;1555(1-3):154-9.

182. Mari M, Morales A, Colell A, Garcia-Ruiz C, Fernandez-Checa JC. Mitochondrial glutathione, a key survival antioxidant. Antioxidants \& redox signaling. 2009;11(11):2685-700.

183. Besouw M, Masereeuw R, van den Heuvel L, Levtchenko E. Cysteamine: an old drug with new potential. Drug discovery today. 2013;18(15-16):785-92.

184. Neymotin A, Calingasan NY, Wille E, Naseri N, Petri S, Damiano M, et al. Neuroprotective effect of Nrf2/ARE activators, CDDO ethylamide and CDDO trifluoroethylamide, in a mouse model of amyotrophic lateral sclerosis. Free Radic Biol Med. 2011;51(1):88-96.

185. Reisman SA, Goldsberry AR, Lee CY, O'Grady ML, Proksch JW, Ward KW, et al. Topical application of RTA 408 lotion activates Nrf2 in human skin and is well-tolerated by healthy human volunteers. BMC dermatology. 2015;15:10.

186. Probst BL, Trevino I, McCauley L, Bumeister R, Dulubova I, Wigley WC, et al. RTA 408, A Novel Synthetic Triterpenoid with Broad Anticancer and Anti-Inflammatory Activity. PLoS One. 2015;10(4):e0122942.

187. Koufaki M. Therapeutic applications of lipoic acid: a patent review (2011 - 2014). Expert opinion on therapeutic patents. 2014;24(9):993-1005.

188. Gupta SC, Prasad S, Kim JH, Patchva S, Webb LJ, Priyadarsini IK, et al. Multitargeting by curcumin as revealed by molecular interaction studies. Natural product reports. 2011;28(12):1937-55.

189. Trujillo J, Granados-Castro LF, Zazueta C, Anderica-Romero AC, Chirino YI, Pedraza-Chaverri J. Mitochondria as a target in the therapeutic properties of curcumin. Archiv der Pharmazie. 2014;347(12):873-84.

190. de Haas R, Das D, Garanto A, Renkema HG, Greupink R, van den Broek P, et al. Therapeutic effects of the mitochondrial ROS-redox modulator KH176 in a mammalian model of Leigh Disease. Scientific reports. 2017;7(1):11733.

191. Li J, Kim SG, Blenis J. Rapamycin: one drug, many effects. Cell Metab. 2014;19(3):373-9.

192. Cunningham JT, Rodgers JT, Arlow DH, Vazquez F, Mootha VK, Puigserver P. mTOR controls mitochondrial oxidative function through a YY1-PGC-1alpha transcriptional complex. Nature. 2007; 450(7170):736-40.

193. Johnson SC, Martin GM, Rabinovitch PS, Kaeberlein M. Preserving youth: does rapamycin deliver? Sci Transl Med. 2013;5(211):211fs40. 
194. Johnson SC, Yanos ME, Kayser EB, Quintana A, Sangesland M, Castanza A, et al. mTOR inhibition alleviates mitochondrial disease in a mouse model of Leigh syndrome. Science (New York, NY). 2013;342(6165):1524-8.

195. Motoi Y, Shimada K, Ishiguro K, Hattori N. Lithium and autophagy. ACS chemical neuroscience. 2014;5(6):434-42.

196. Sarkar S, Floto RA, Berger Z, Imarisio S, Cordenier A, Pasco M, et al. Lithium induces autophagy by inhibiting inositol monophosphatase. The Journal of cell biology. 2005;170(7):1101-11.

197. Sarkar S, Krishna G, Imarisio S, Saiki S, O'Kane CJ, Rubinsztein DC. A rational mechanism for combination treatment of Huntington's disease using lithium and rapamycin. Hum Mol Genet. 2008;17(2):170-8.

198. Gerards M, Kamps R, van Oevelen J, Boesten I, Jongen E, de Koning B, et al. Exome sequencing reveals a novel Moroccan founder mutation in SLC19A3 as a new cause of early-childhood fatal Leigh syndrome. Brain. 2013;136(Pt 3):882-90.

199. Ortigoza-Escobar JD, Serrano M, Molero M, Oyarzabal A, Rebollo M, Muchart J, et al. Thiamine transporter-2 deficiency: outcome and treatment monitoring. Orphanet J Rare Dis. 2014;9:92.

200. Schiff M, Veauville-Merllie A, Su CH, Tzagoloff A, Rak M, Ogier de Baulny H, et al. SLC25A32 Mutations and Riboflavin-Responsive Exercise Intolerance. N Engl J Med. 2016;374(8):795-7.

201. Hellebrekers D, Sallevelt S, Theunissen TEJ, Hendrickx ATM, Gottschalk RW, Hoeijmakers JGJ, et al. Novel SLC25A32 mutation in a patient with a severe neuromuscular phenotype. European journal of human genetics : EJHG. 2017;25(7):886-8.

202. Quinzii $\mathrm{CM}$, Hirano M. Primary and secondary $\mathrm{CoQ}(10)$ deficiencies in humans. BioFactors (Oxford, England). 2011;37(5):361-5.

203. Patel KP, O'Brien TW, Subramony SH, Shuster J, Stacpoole PW. The spectrum of pyruvate dehydrogenase complex deficiency: clinical, biochemical and genetic features in 371 patients. Mol Genet Metab. 2012;106(3):385-94.

204. Vila MR, Segovia-Silvestre T, Gamez J, Marina A, Naini AB, Meseguer A, et al. Reversion of mtDNA depletion in a patient with TK2 deficiency. Neurology. 2003;60(7):1203-5.

205. Bulst S, Holinski-Feder E, Payne B, Abicht A, Krause S, Lochmuller H, et al. In vitro supplementation with deoxynucleoside monophosphates rescues mitochondrial DNA depletion. Mol Genet Metab. 2012;107(1-2):95-103.

206. Bulst S, Abicht A, Holinski-Feder E, Muller-Ziermann S, Koehler U, Thirion C, et al. In vitro supplementation with dAMP/dGMP leads to partial restoration of mtDNA levels in mitochondrial depletion syndromes. Hum Mol Genet. 2009;18(9):1590-9.

207. Camara Y, Gonzalez-Vioque E, Scarpelli M, Torres-Torronteras J, Caballero A, Hirano M, et al. Administration of deoxyribonucleosides or inhibition of their catabolism as a pharmacological approach for mitochondrial DNA depletion syndrome. Hum Mol Genet. 2014;23(9):2459-67.

208. Garone C, Garcia-Diaz B, Emmanuele V, Lopez LC, Tadesse S, Akman HO, et al. Deoxypyrimidine monophosphate bypass therapy for thymidine kinase 2 deficiency. EMBO molecular medicine. 2014;6(8):1016-27.

209. Hinttala R, Sasarman F, Nishimura T, Antonicka H, Brunel-Guitton C, Schwartzentruber J, et al. An $\mathrm{N}$-terminal formyl methionine on COX 1 is required for the assembly of cytochrome c oxidase. Hum Mol Genet. 2015;24(14):4103-13.

210. Haack TB, Danhauser K, Haberberger B, Hoser J, Strecker V, Boehm D, et al. Exome sequencing identifies ACAD9 mutations as a cause of complex I deficiency. Nat Genet. 2010;42(12):1131-4.

211. Theunissen TEJ, Gerards M, Hellebrekers D, van Tienen FH, Kamps R, Sallevelt S, et al. Selection and Characterization of Palmitic Acid Responsive Patients with an OXPHOS Complex I Defect. Frontiers in molecular neuroscience. 2017;10:336.

212. Minczuk M, Papworth MA, Miller JC, Murphy MP, Klug A. Development of a single-chain, quasidimeric zinc-finger nuclease for the selective degradation of mutated human mitochondrial DNA. Nucleic Acids Res. 2008;36(12):3926-38. 
213. Bacman SR, Williams SL, Pinto M, Peralta S, Moraes CT. Specific elimination of mutant mitochondrial genomes in patient-derived cells by mitoTALENs. Nature medicine. 2013;19(9):1111-3.

214. Gammage PA, Rorbach J, Vincent Al, Rebar EJ, Minczuk M. Mitochondrially targeted ZFNs for selective degradation of pathogenic mitochondrial genomes bearing large-scale deletions or point mutations. EMBO molecular medicine. 2014;6(4):458-66.

215. Bacman SR, Williams SL, Duan D, Moraes CT. Manipulation of mtDNA heteroplasmy in all striated muscles of newborn mice by AAV9-mediated delivery of a mitochondria-targeted restriction endonuclease. Gene therapy. 2012;19(11):1101-6.

216. Srivastava S, Moraes $\mathrm{CT}$. Manipulating mitochondrial DNA heteroplasmy by a mitochondrially targeted restriction endonuclease. Hum Mol Genet. 2001;10(26):3093-9.

217. Alexeyev MF, Venediktova N, Pastukh V, Shokolenko I, Bonilla G, Wilson GL. Selective elimination of mutant mitochondrial genomes as therapeutic strategy for the treatment of NARP and MILS syndromes. Gene therapy. 2008;15(7):516-23.

218. Bacman SR, Williams SL, Pinto M, Moraes CT. The use of mitochondria-targeted endonucleases to manipulate mtDNA. Methods in enzymology. 2014;547:373-97.

219. Klug A. The discovery of zinc fingers and their development for practical applications in gene regulation and genome manipulation. Quarterly reviews of biophysics. 2010;43(1):1-21.

220. Ramalingam S, Kandavelou K, Rajenderan R, Chandrasegaran S. Creating designed zinc-finger nucleases with minimal cytotoxicity. Journal of molecular biology. 2011;405(3):630-41.

221. Radecke S, Radecke F, Cathomen T, Schwarz K. Zinc-finger nuclease-induced gene repair with oligodeoxynucleotides: wanted and unwanted target locus modifications. Molecular therapy : the journal of the American Society of Gene Therapy. 2010;18(4):743-53.

222. Ellis BL, Hirsch ML, Porter SN, Samulski RJ, Porteus MH. Zinc-finger nuclease-mediated gene correction using single AAV vector transduction and enhancement by Food and Drug Administration-approved drugs. Gene therapy. 2013;20(1):35-42.

223. Moscou MJ, Bogdanove AJ. A simple cipher governs DNA recognition by TAL effectors. Science (New York, NY). 2009;326(5959):1501.

224. Christian M, Cermak T, Doyle EL, Schmidt C, Zhang F, Hummel A, et al. Targeting DNA doublestrand breaks with TAL effector nucleases. Genetics. 2010;186(2):757-61.

225. Reddy P, Ocampo A, Suzuki K, Luo J, Bacman SR, Williams SL, et al. Selective elimination of mitochondrial mutations in the germline by genome editing. Cell. 2015;161(3):459-69.

226. Hsu PD, Lander ES, Zhang F. Development and applications of CRISPR-Cas9 for genome engineering. Cell. 2014;157(6):1262-78.

227. Cox DB, Platt RJ, Zhang F. Therapeutic genome editing: prospects and challenges. Nature medicine. 2015;21(2):121-31.

228. Maeder ML, Thibodeau-Beganny S, Osiak A, Wright DA, Anthony RM, Eichtinger M, et al. Rapid "open-source" engineering of customized zinc-finger nucleases for highly efficient gene modification. Molecular cell. 2008;31(2):294-301.

229. Sander JD, Cade L, Khayter C, Reyon D, Peterson RT, Joung JK, et al. Targeted gene disruption in somatic zebrafish cells using engineered TALENs. Nature biotechnology. 2011;29(8):697-8.

230. Juillerat A, Dubois G, Valton J, Thomas S, Stella S, Marechal A, et al. Comprehensive analysis of the specificity of transcription activator-like effector nucleases. Nucleic Acids Res. 2014;42(8):5390402.

231. Li HL, Fujimoto N, Sasakawa N, Shirai S, Ohkame T, Sakuma T, et al. Precise correction of the dystrophin gene in duchenne muscular dystrophy patient induced pluripotent stem cells by TALEN and CRISPR-Cas9. Stem cell reports. 2015;4(1):143-54.

232. Liu C, Zhang L, Liu H, Cheng K. Delivery strategies of the CRISPR-Cas9 gene-editing system for therapeutic applications. Journal of controlled release : official journal of the Controlled Release Society. 2017;266:17-26. 
233. Schwank G, Koo BK, Sasselli V, Dekkers JF, Heo I, Demircan T, et al. Functional repair of CFTR by CRISPR/Cas9 in intestinal stem cell organoids of cystic fibrosis patients. Cell stem cell. 2013;13(6):653-8.

234. Xu P, Tong $\mathrm{Y}$, Liu XZ, Wang TT, Cheng L, Wang BY, et al. Both TALENs and CRISPR/Cas9 directly target the HBB IVS2-654 (C > T) mutation in beta-thalassemia-derived iPSCs. Scientific reports. 2015;5:12065.

235. Hu Z, Yu L, Zhu D, Ding W, Wang X, Zhang C, et al. Disruption of HPV16-E7 by CRISPR/Cas system induces apoptosis and growth inhibition in HPV16 positive human cervical cancer cells. Biomed Res Int. 2014;2014:612823.

236. Mandal PK, Ferreira LM, Collins R, Meissner TB, Boutwell CL, Friesen M, et al. Efficient ablation of genes in human hematopoietic stem and effector cells using CRISPR/Cas9. Cell stem cell. 2014;15(5):643-52.

237. Suzuki K, Yu C, Qu J, Li M, Yao X, Yuan T, et al. Targeted gene correction minimally impacts wholegenome mutational load in human-disease-specific induced pluripotent stem cell clones. Cell stem cell. 2014;15(1):31-6.

238. Xie F, Ye L, Chang JC, Beyer Al, Wang J, Muench MO, et al. Seamless gene correction of betathalassemia mutations in patient-specific iPSCs using CRISPR/Cas9 and piggyBac. Genome Res. 2014;24(9):1526-33.

239. Li D, Qiu Z, Shao Y, Chen Y, Guan Y, Liu M, et al. Heritable gene targeting in the mouse and rat using a CRISPR-Cas system. Nature biotechnology. 2013;31(8):681-3.

240. Fogleman S, Santana C, Bishop C, Miller A, Capco DG. CRISPR/Cas9 and mitochondrial gene replacement therapy: promising techniques and ethical considerations. American journal of stem cells. 2016;5(2):39-52.

241. Yin H, Xue W, Chen S, Bogorad RL, Benedetti E, Grompe M, et al. Genome editing with Cas9 in adult mice corrects a disease mutation and phenotype. Nature biotechnology. 2014;32(6):5513.

242. Ohmori T, Nagao Y, Mizukami H, Sakata A, Muramatsu SI, Ozawa K, et al. CRISPR/Cas9-mediated genome editing via postnatal administration of AAV vector cures haemophilia B mice. Scientific reports. 2017;7(1):4159.

243. Bengtsson NE, Hall JK, Odom GL, Phelps MP, Andrus CR, Hawkins RD, et al. Muscle-specific CRISPR/Cas9 dystrophin gene editing ameliorates pathophysiology in a mouse model for Duchenne muscular dystrophy. Nature communications. 2017;8:14454.

244. Jo A, Ham S, Lee GH, Lee YI, Kim S, Lee YS, et al. Efficient Mitochondrial Genome Editing by CRISPR/Cas9. Biomed Res Int. 2015;2015:305716.

245. Hyrup B, Nielsen PE. Peptide nucleic acids (PNA): synthesis, properties and potential applications. Bioorganic \& medicinal chemistry. 1996;4(1):5-23.

246. Egholm M, Buchardt O, Christensen L, Behrens C, Freier SM, Driver DA, et al. PNA hybridizes to complementary oligonucleotides obeying the Watson-Crick hydrogen-bonding rules. Nature. 1993;365(6446):566-8.

247. Demidov VV, Potaman VN, Frank-Kamenetskii MD, Egholm M, Buchard O, Sonnichsen SH, et al. Stability of peptide nucleic acids in human serum and cellular extracts. Biochemical pharmacology. 1994;48(6):1310-3.

248. Muratovska A, Lightowlers RN, Taylor RW, Turnbull DM, Smith RA, Wilce JA, et al. Targeting peptide nucleic acid (PNA) oligomers to mitochondria within cells by conjugation to lipophilic cations: implications for mitochondrial DNA replication, expression and disease. Nucleic Acids Res. 2001;29(9):1852-63.

249. Chinnery PF, Taylor RW, Diekert K, Lill R, Turnbull DM, Lightowlers RN. Peptide nucleic acid delivery to human mitochondria. Gene therapy. 1999;6(12):1919-28. 
250. Gray GD, Basu S, Wickstrom E. Transformed and immortalized cellular uptake of oligodeoxynucleoside phosphorothioates, 3'-alkylamino oligodeoxynucleotides, 2'-0-methyl oligoribonucleotides, oligodeoxynucleoside methylphosphonates, and peptide nucleic acids. Biochemical pharmacology. 1997;53(10):1465-76.

251. Nielsen PE, Egholm M. An introduction to peptide nucleic acid. Current issues in molecular biology. 1999;1(1-2):89-104.

252. Ljungstrom T, Knudsen $\mathrm{H}$, Nielsen PE. Cellular uptake of adamantyl conjugated peptide nucleic acids. Bioconjugate chemistry. 1999;10(6):965-72.

253. Pooga M, Soomets U, Hallbrink M, Valkna A, Saar K, Rezaei K, et al. Cell penetrating PNA constructs regulate galanin receptor levels and modify pain transmission in vivo. Nature biotechnology. 1998;16(9):857-61.

254. Kyriakouli DS, Boesch P, Taylor RW, Lightowlers RN. Progress and prospects: gene therapy for mitochondrial DNA disease. Gene therapy. 2008;15(14):1017-23.

255. Ran FA, Hsu PD, Wright J, Agarwala V, Scott DA, Zhang F. Genome engineering using the CRISPRCas9 system. Nat Protoc. 2013;8(11):2281-308.

256. Long C, Amoasii L, Bassel-Duby R, Olson EN. Genome Editing of Monogenic Neuromuscular Diseases: A Systematic Review. JAMA neurology. 2016;73(11):1349-55.

257. Xiao-Jie L, Hui-Ying X, Zun-Ping K, Jin-Lian C, Li-Juan J. CRISPR-Cas9: a new and promising player in gene therapy. J Med Genet. 2015;52(5):289-96.

258. Tu Z, Yang W, Yan S, Yin A, Gao J, Liu X, et al. Promoting Cas9 degradation reduces mosaic mutations in non-human primate embryos. Scientific reports. 2017;7:42081.

259. Tu Z, Yang W, Yan S, Guo X, Li XJ. CRISPR/Cas9: a powerful genetic engineering tool for establishing large animal models of neurodegenerative diseases. Molecular neurodegeneration. 2015;10:35.

260. Ma H, Marti-Gutierrez N, Park SW, Wu J, Lee Y, Suzuki K, et al. Correction of a pathogenic gene mutation in human embryos. Nature. 2017;548(7668):413-9.

261. Kotterman MA, Schaffer DV. Engineering adeno-associated viruses for clinical gene therapy. Nature reviews Genetics. 2014;15(7):445-51.

262. Yang $Y$, Wang $L$, Bell $P$, McMenamin D, He Z, White J, et al. A dual AAV system enables the Cas9-mediated correction of a metabolic liver disease in newborn mice. Nature biotechnology. 2016;34(3):334-8.

263. Yin $\mathrm{H}$, Song CQ, Dorkin JR, Zhu LJ, Li Y, Wu Q, et al. Therapeutic genome editing by combined viral and non-viral delivery of CRISPR system components in vivo. Nature biotechnology. 2016;34(3):328-33.

264. Yu H, Koilkonda RD, Chou TH, Porciatti V, Ozdemir SS, Chiodo V, et al. Gene delivery to mitochondria by targeting modified adenoassociated virus suppresses Leber's hereditary optic neuropathy in a mouse model. Proc Natl Acad Sci U S A. 2012;109(20):E1238-47.

265. Wu Y, Liang D, Wang Y, Bai M, Tang W, Bao S, et al. Correction of a genetic disease in mouse via use of CRISPR-Cas9. Cell stem cell. 2013;13(6):659-62.

266. Tabebordbar M, Zhu K, Cheng JKW, Chew WL, Widrick JJ, Yan WX, et al. In vivo gene editing in dystrophic mouse muscle and muscle stem cells. Science (New York, NY). 2016;351(6271):40711.

267. Nelson CE, Hakim CH, Ousterout DG, Thakore PI, Moreb EA, Castellanos Rivera RM, et al. In vivo genome editing improves muscle function in a mouse model of Duchenne muscular dystrophy. Science (New York, NY). 2016;351(6271):403-7.

268. Kumar SR, Markusic DM, Biswas M, High KA, Herzog RW. Clinical development of gene therapy: results and lessons from recent successes. Molecular therapy Methods \& clinical development. 2016;3:16034. 
269. Herzog RW, Cao O, Srivastava A. Two decades of clinical gene therapy--success is finally mounting. Discovery medicine. 2010;9(45):105-11.

270. Bank A. Human somatic cell gene therapy. BioEssays : news and reviews in molecular, cellular and developmental biology. 1996;18(12):999-1007.

271. Nayerossadat N, Maedeh T, Ali PA. Viral and nonviral delivery systems for gene delivery. Advanced biomedical research. 2012;1:27.

272. Ramamoorth $M$, Narvekar A. Non viral vectors in gene therapy- an overview. Journal of clinical and diagnostic research : JCDR. 2015;9(1):GE01-6.

273. Aiuti A, Biasco L, Scaramuzza S, Ferrua F, Cicalese MP, Baricordi C, et al. Lentiviral hematopoietic stem cell gene therapy in patients with Wiskott-Aldrich syndrome. Science (New York, NY). 2013;341(6148):1233151.

274. Lai CM, Lai YK, Rakoczy PE. Adenovirus and adeno-associated virus vectors. DNA and cell biology. 2002;21(12):895-913.

275. Flotte TR. Gene therapy: the first two decades and the current state-of-the-art. Journal of cellular physiology. 2007;213(2):301-5.

276. Kay MA, Manno CS, Ragni MV, Larson PJ, Couto LB, McClelland A, et al. Evidence for gene transfer and expression of factor IX in haemophilia B patients treated with an AAV vector. Nat Genet. 2000;24(3):257-61.

277. Di Meo I, Auricchio A, Lamperti C, Burlina A, Viscomi C, Zeviani M. Effective AAV-mediated gene therapy in a mouse model of ethylmalonic encephalopathy. EMBO molecular medicine. 2012;4(9):1008-14.

278. Torres-Torronteras J, Viscomi C, Cabrera-Perez R, Camara Y, Di Meo I, Barquinero J, et al. Gene therapy using a liver-targeted AAV vector restores nucleoside and nucleotide homeostasis in a murine model of MNGIE. Molecular therapy : the journal of the American Society of Gene Therapy. 2014;22(5):901-7.

279. Bouaita A, Augustin S, Lechauve C, Cwerman-Thibault H, Benit P, Simonutti M, et al. Downregulation of apoptosis-inducing factor in Harlequin mice induces progressive and severe optic atrophy which is durably prevented by AAV2-AIF1 gene therapy. Brain. 2012;135(Pt 1):3552.

280. Bottani E, Giordano C, Civiletto G, Di Meo I, Auricchio A, Ciusani E, et al. AAV-mediated liverspecific MPV17 expression restores mtDNA levels and prevents diet-induced liver failure. Molecular therapy : the journal of the American Society of Gene Therapy. 2014;22(1):10-7.

281. Bokori-Brown M, Holt IJ. Expression of algal nuclear ATP synthase subunit 6 in human cells results in protein targeting to mitochondria but no assembly into ATP synthase. Rejuvenation research. 2006;9(4):455-69.

282. Guy J, Qi X, Pallotti F, Schon EA, Manfredi G, Carelli V, et al. Rescue of a mitochondrial deficiency causing Leber Hereditary Optic Neuropathy. Ann Neurol. 2002;52(5):534-42.

283. Ellouze S, Augustin S, Bouaita A, Bonnet C, Simonutti M, Forster V, et al. Optimized allotopic expression of the human mitochondrial ND4 prevents blindness in a rat model of mitochondrial dysfunction. Am J Hum Genet. 2008;83(3):373-87.

284. Figueroa-Martinez F, Vazquez-Acevedo M, Cortes-Hernandez P, Garcia-Trejo JJ, Davidson E, King MP, et al. What limits the allotopic expression of nucleus-encoded mitochondrial genes? The case of the chimeric Cox3 and Atp6 genes. Mitochondrion. 2011;11(1):147-54.

285. Lunn JS, Sakowski SA, Hur J, Feldman EL. Stem cell technology for neurodegenerative diseases. Ann Neurol. 2011;70(3):353-61.

286. Nightingale H, Pfeffer G, Bargiela D, Horvath R, Chinnery PF. Emerging therapies for mitochondrial disorders. Brain. 2016;139(Pt 6):1633-48.

287. Hirano M, Marti R, Casali C, Tadesse S, Uldrick T, Fine B, et al. Allogeneic stem cell transplantation corrects biochemical derangements in MNGIE. Neurology. 2006;67(8):1458-60. 
288. Filosto M, Scarpelli M, Tonin P, Lucchini G, Pavan F, Santus F, et al. Course and management of allogeneic stem cell transplantation in patients with mitochondrial neurogastrointestinal encephalomyopathy. J Neurol. 2012;259(12):2699-706.

289. Sicurelli F, Carluccio MA, Toraldo F, Tozzi M, Bucalossi A, Lenoci $M$, et al. Clinical and biochemical improvement following HSCT in a patient with MNGIE: 1-year follow-up. J Neurol. 2012;259(9):1985-7.

290. Hussein E. Non-myeloablative bone marrow transplant and platelet infusion can transiently improve the clinical outcome of mitochondrial neurogastrointestinal encephalopathy: a case report. Transfusion and apheresis science : official journal of the World Apheresis Association : official journal of the European Society for Haemapheresis. 2013;49(2):208-11.

291. Moran NF, Bain MD, Muqit MM, Bax BE. Carrier erythrocyte entrapped thymidine phosphorylase therapy for MNGIE. Neurology. 2008;71(9):686-8.

292. Bax BE, Bain MD, Scarpelli M, Filosto M, Tonin P, Moran N. Clinical and biochemical improvements in a patient with MNGIE following enzyme replacement. Neurology. 2013;81(14):1269-71.

293. Halter JP, Michael W, Schupbach M, Mandel H, Casali C, Orchard K, et al. Allogeneic haematopoietic stem cell transplantation for mitochondrial neurogastrointestinal encephalomyopathy. Brain. 2015;138(Pt 10):2847-58.

294. Spendiff S, Reza M, Murphy JL, Gorman G, Blakely EL, Taylor RW, et al. Mitochondrial DNA deletions in muscle satellite cells: implications for therapies. Hum Mol Genet. 2013;22(23):473947.

295. Clark KM, Bindoff LA, Lightowlers RN, Andrews RM, Griffiths PG, Johnson MA, et al. Reversal of a mitochondrial DNA defect in human skeletal muscle. Nat Genet. 1997;16(3):222-4. 

SUMMARY 
Summary 
Mitochondrial disorders are a group of inheritable metabolic disorders that affect approximately 1 in 5,000 individuals, and result from an insufficient capacity to produce ATP due to abnormalities in the oxidative phosphorylation (OXPHOS) system. As a result, a broad variety of, especially, high energy demanding organs can be affected, with often muscle, brain and liver involved. Mitochondrial disorders are clinically and genetically heterogeneous, as a specific gene defect can be pleiotropic in its clinical manifestations, or a similar disease phenotype can result from different gene defects. Besides, mitochondrial disease can be caused by both, mitochondrial DNA (mtDNA) or nuclear gene defects, where still a considerable part of the nuclear DNA defects and genes remain to be discovered. This heterogeneity complicates the identification of the genetic basis of mitochondrial disease. A complete genetic characterization is crucial, not only to establish a genetic diagnosis in the patient, or to prevent disease transmission towards offspring, but also to improve our understanding of the pathophysiological basis of mitochondrial disease, with the ultimate aim to develop effective treatment options (chapter 1). The development of next generation sequencing (NGS) has revolutionized the identification of genetic defects in mitochondrial patients. Our central hypothesis was that whole exome sequencing would enable us to identify the underlying genetic cause, characterize the pathophysiological mechanisms and unravel possible targets for therapeutic intervention in patients with mitochondrial disease, who could not be solved using conventional targeted gene analysis methods.

Our first aim was to develop and implement whole exome sequencing and novel bioinformatics approaches for the identification of gene defects in patients with a likely or possible mitochondrial disease. In chapter $\mathbf{2}$ we described a two-step NGS-based approach in a cohort of 117 patients, mostly children, in who a genetic cause was likely. Patients either had a high likelihood of having a mitochondrial disorder, as based on the Nijmegen clinical criteria and/or involvement of OXPHOS deficiencies, or had a disease phenotype in which mitochondrial defects could be part of the differential diagnosis. Patients were screened for mtDNA defects and, if excluded, analyzed by whole exome sequencing (WES). We filtered for autosomal recessive and $X$-linked recessive mutations in families with multiple affected siblings and/or consanguineous parents. Non-consanguineous families with a single patient were additionally screened for autosomal and X-linked dominant mutations in a predefined gene set. We detected and quantified mtDNA point-mutations and indels in $21 \%$ of the patient-cohort, and identified disease-causing exonic mutations in $49 \%$ of the patients, implying an overall diagnostic yield of $69 \%$. We showed that $30 \%$ of the disease-causing genes in mitochondrial WES-patients were not included in the MitoCarta database at the time of discovery, and therefore would have been missed with targeted, gene panel based sequencing approaches. WES is therefore a comprehensive and unbiased approach to establish a genetic diagnosis in mitochondrial disease. In chapter $\mathbf{3}$ we highlight the 
importance of following-up all variants identified by WES that could be related to the patient's clinical symptoms. WES of two patients, suffering from a broad variety of clinical symptoms, revealed that the consanguineous patient had three homozygous mutations, underlying a blended phenotype, and the non-consanguineous patient had a compound heterozygous mutation combined with a dominant de novo mutation, underlying a composite clinical manifestation. Heterogeneous clinical phenotypes may therefore not always be caused by a single gene defect, especially in consanguineous families, where homozygosity regions increase the risk of inheriting multiple recessive genetic defects. Whereas candidate gene approaches often fall short in explaining the entire clinical picture, and require multiple rounds of clinical investigations, we show that WES provides an unbiased solution for fully resolving composite or blended phenotypes that arise from multiple gene defects. When establishing a genetic diagnosis in heterogeneous disease, clinical characterization and WES can go hand-in-hand. In chapter 4 we demonstrated that clinical data could be essential, not only to guide WES variant selection and define the clinical severity of a genetic defect, but also to identify other patients with defects in the same gene. In an infant patient with pronounced neurological features, WES resulted in identification of a novel homozygous CLPP mutation. Based on the gene defect and clinical symptoms, the diagnosis Perrault syndrome type 3 (PRLTS3) was established. The patient's brain-MRI showed specific abnormalities, and was used to identify similar patients in the Amsterdam brain-MRI database, containing over 3000 unclassified leukoencephalopathy cases. In two unrelated patients with similar MRI abnormalities, novel CLPP missense mutations were identified. Our data therefore show that similarity in brain-MRI patterns can be used to identify novel PRLTS3 patients, especially during early disease stages, when only part of the disease manifestations is present.

Our second aim was to characterize the functional effect of mutations in known and novel genes in patient cell lines and zebrafish. In chapter 5 we investigated a complex I deficient patient with exercise intolerance, in whom dietary studies showed increased muscle endurance on a high-fat diet compared to a high-carbohydrate diet. We performed WES to characterize the genetic defect and identified a pathogenic mutation in the TMEM126B gene, encoding an early assembly factor of complex I. A complementation study in patient fibroblasts confirmed that the TMEM126B defect caused the complex I deficiency, which was an incomplete assembly of the peripheral arm of complex I. Based on in vitro analysis of the patient's fibroblasts, we showed that treatment with the saturated fatty acid palmitic acid resulted in an increase in maximal OXPHOS capacity, indicating responsiveness of the patient cells to palmitic acid and supporting the clinical improvements in the patient. Our third aim was to identify, based on the genes and pathways involved, novel therapeutic targets. Based on the promising results in the TMEM126B patient, we therefore performed the same assay on fibroblasts from patients with different complex I gene defects, including 
defects in the core-subunits and assembly factors of complex I. We demonstrated that also other gene defects involved in early assembly of complex I (NDUFS7 and NDUFAF5) were responsive to palmitic acid, whereas late assembly defects seemed non-responsive. Our data showed at a clinical and biochemical level that a high fat diet could be beneficial for complex I patients and that our cell line assay can be an easy tool for the selection of patients, who might potentially benefit from such a therapeutic diet (chapter 5).

In chapter 6 we performed WES in a patient with Hirschprung disease (HSCR) and leftsided ptosis, and identified a novel missense mutation in the ERBB2 gene. Our patient had striking phenotypic similarity with a previously reported conditional erbB2 mutant mouse, in which a lack of functional erbB2 expression mimicked the human HSCR phenotype and also resulted in ptosis. The mutation caused a likely pathogenic substitution in the Juxta-membrane domain of the ERBB2 receptor, but could also create a potential cryptic donor splice site. Yet, RNA data showed that cryptic splicing was not activated, and also no non-sense mediated decay was measured in the patient's fibroblasts. Besides, ERBB2 in vitro overexpression studies showed no indications that ERBB2 activity was altered due to the mutation or that membrane localization was affected. Therefore, we could not unequivocally demonstrate that the variant inactivated ERBB2 based on our in vitro studies. Evidence for a definite role for ERBB2 in HSCR therefore awaits further functional validation, especially based on the effect on the enteric nerve system (ENS). The Phox- $2 b$ reporter zebrafish line will be used to analyze the ENS after CRISPR/Cas9 based knock-down/out of ERBB2. Although the zebrafish model has been validated as a useful model to study nuclear gene mutations, data on mtDNA, and the mechanisms involved in mtDNA segregation, are lacking. Therefore, in chapter 7 we studied the mtDNA bottleneck in zebrafish to elucidate size, timing and variation in germline and non-germline cells. Mature zebrafish oocytes contain $\sim 19.0 \times 10^{6}$ mtDNA molecules with high variation between oocytes. We showed that during embryogenesis, the mtDNA copy number decreases to 170 mtDNA molecules per primordial germ cell (PGC), a number similar to mammals, and to $\sim 50$ per non-PGC. These bottlenecks occur at the same developmental stage, implying considerable variation in mtDNA copy number in (non-)PGCs of the same female, dictated by variation in the mature oocyte. Therefore, bottleneck differences between germline and non-germline cells, due to early differentiation of PGCs, may account for different distribution patterns of familial mtDNA mutations in humans. 
In chapter $\mathbf{8}$ we review the different genetic models that are used to study mitochondrial disease. Especially with the involvement of WES, there is demand for a genetic model that allows the testing of variant pathogenicity in a relatively quick and straightforward manner, specifically for variants of unknown significance (VUS) and variants in novel genes. Suitable read-out models to test variant pathogenicity can be based on in silico analysis, in vitro testing or in vivo models. We give an overview of different strategies to functionally validate mutation pathogenicity in mitochondrial disease, and discuss the advantages and disadvantages of each model. Yet, the choice for a certain genetic-model is ultimately defined by the research question, implying that there exists no single model that fits all demands. Despite the current successes in resolving the genetic causes of mitochondrial disease, the translation into effective treatment strategies is still limited, where current treatments options are mainly supportive. This is in part due the enormous genetic and phenotypic heterogeneity in mitochondrial disease. We discuss the different generic treatment strategies, based on exercise training, diets and small molecule pharmaceuticals (stimulators of biogenesis, ETC function and ROS scavenging), that aim to improve mitochondrial capacity by increasing mitochondrial biogenesis or improving ETC function. In contrast to generic treatment, tailored treatment strategies specifically target the genetic or metabolic defect in the patient. Tailored treatment covers the supplementation of specific enzymes, substrates or nucleotides to restore the metabolic deficiency, genetic manipulation (shifting mtDNA heteroplasmy, gene editing of nDNA mutations, gene transfer therapy) and stem cell therapies. Due to the large genetic, clinical and biochemical heterogeneity, it is most likely that a 'one size fits all' therapy will not be possible and that tailored intervention therapies and specific combinations of therapeutics will be needed to effectively target each specific subgroup of mitochondrial disease, eventually going towards personalized medicine.

\section{Conclusion}

We showed that whole-exome sequencing enabled us the identify the genetic cause in $69 \%$ of the patients with mitochondrial disease, that could not be solved with conventional sequencing methods. Novel bioinformatics tools, such as WeGET helped us to select promising candidate genes based on co-expression data. WES is therefore the preferred, first strategy to characterize the genetic defect in patients with mitochondrial disease, especially in consanguineous families, where there is an increased risk of inheriting multiple gene defects. Based on complementation studies in patient cell lines, we have been able to characterize and prove the functional consequences of known and novel gene defects. Besides, we have shown that the zebrafish is a powerful model to study mitochondrial disease, not only as a model for nDNA defects, as commonly reported in literature, but also to study mtDNA transmission. The characterization of genes and pathways involved 
in mitochondrial disease has in a limited number of cases led to the identification of novel therapeutic targets, as we showed that treatments based on thiamine, riboflavin or high-fat diets could be beneficial for patients with specific mitochondrial gene defects (SLC19A3, SLC25A32, ACAD9, TMEM126B). This indicates that the treatment of mitochondrial disease will become highly personalized and will require additional experimental approaches. 

Samenvatting 
Mitochondriële aandoeningen behoren tot een groep van overerfbare metabole ziektes, en treffen ongeveer 1 op de 5000 individuen door een onvoldoende capaciteit om ATP te produceren, dit als gevolg van defecten in het oxidatieve fosforylerings systeem (OXPHOS). Vandaar dat een grote verscheidenheid aan vooral veel energie eisende organen, zoals spier, hersenen en lever, kunnen zijn aangedaan. Mitochondriële aandoeningen zijn klinisch en genetisch heterogeen, aangezien een specifiek gendefect pleiotropisch kan zijn in haar klinische manifestaties, en vergelijkbare ziektebeelden kunnen voortkomen uit verschillende genetische fouten. Daarnaast kunnen mitochondriële aandoeningen veroorzaakt worden door zowel defecten in het mitochondriële DNA (mtDNA) als het nucleaire genoom (nDNA). Deze heterogeniteit bemoeilijkt het identificeren van de genetische basis in mitochondriële aandoeningen, waardoor, met name in het nDNA, er nog een aanzienlijk aantal mitochondriële gendefecten ongeïdentificeerd zijn. Een volledige genetische karakterisatie is cruciaal, niet alleen om diagnose te kunnen stellen in de patiënt, of het voorkomen van ziekteoverdracht op volgende generaties, maar ook om de pathofysiologische basis van mitochondriële ziekten beter te begrijpen, met als uiteindelijk doel het ontwikkelen van effectieve behandelingsopties (hoofdstuk 1). De ontwikkeling van next generation sequencing (NGS) heeft gezorgd voor een revolutionaire doorbraak in het identificeren van genetische defecten in mitochondriële patiënten. Onze centrale hypothese was dat whole exome sequencing ons in staat zou stellen de onderliggende genetische oorzaak the identificeren, de pathofysiologische mechanismen te karakteriseren, en tot het aanduiden van mogelijke 'target' moleculen of processen voor therapeutische interventie in mitochondriële patiënten. Hierbij gaat het om patiënten bij wie de genetische oorzaak niet kon worden vastgesteld met behulp van conventionele gerichte gen analyse.

Ons eerste doel was het implementeren van whole exome sequencing en nieuwe bioinformatica benaderingen om de genetisch defecten te identificeren in patiënten met waarschijnlijk, of mogelijk een mitochondriële aandoening. In hoofdstuk $\mathbf{2}$ beschrijven we een twee-staps NGS benadering in een cohort met 117 patiënten, voornamelijk kinderen, met meest waarschijnlijk een genetische component als oorzaak van de aandoening. Patiënten hadden ofwel hoge verdenking op een mitochondriële aandoening op basis van de Nijmegen klinische criteria en/of de betrokkenheid van een OXPHOS deficiëntie, of ze hadden een ziektebeeld waarbij een mitochondrieel defect onderdeel zou kunnen zijn van de differentiaal diagnostiek. Patiënten werden gescreend voor mtDNA defecten en, indien dit werd uitgesloten, geanalyseerd met behulp van whole exome sequencing (WES). In families met meerdere aangedane kinderen en/of met consanguine ouders werd WES data gefilterd voor autosomaal recessieve en X-chromosomaal gebonden recessieve mutaties. In families zonder consanguine achtergrond en met slecht een enkele aangedane patiënt, werd WES data daarnaast ook gefilterd voor autosomaal en X-chromosomaal gebonden 
dominante mutaties in een vooraf bepaalde groep van mitochondriële genen (genen waarin dominante mutaties eerder zijn gerapporteerd). In $21 \%$ van het patiënt cohort hebben we mtDNA puntmutaties of deleties gedetecteerd en gekwantificeerd, en in $49 \%$ van de patiënten hebben we een ziekte veroorzakende mutatie in het exoom geïdentificeerd, wat neerkomt op een totale diagnostische opbrengst van $69 \%$. Daarbij lieten we zien dat $30 \%$ van de ziekte veroorzakende genen in mitochondriële WES-patiënten niet waren geïncludeerd in de MitoCarta database op het moment van identificatie, en daarom zouden worden gemist met gerichte, op "gene panels" gebaseerde sequencing benaderingen. WES is daarom een volledige en accurate benadering om een genetische diagnose te stellen in mitochondriële aandoeningen. In hoofdstuk $\mathbf{3}$ tonen we het belang aan om alle varianten welke zijn geïdentificeerd met WES op te volgen, totdat alle individuele symptomen in de patiënt kunnen worden verklaard. In twee patiënten met een brede variëteit aan klinische symptomen toonde WES aan dat meerdere gendefecten ten grondslag lagen aan het ziektebeeld. In de consanguine patiënt werden drie verschillende homozygote mutaties geïdentificeerd welke een "blended" ziektebeeld veroorzaakten, gekenmerkt door een overlap in symptomen veroorzaakt vanuit de verschillende individuele gendefecten. De patiënt van niet-consanguine ouders had een compound heterozygote mutatie gecombineerd met een dominante de novo mutatie welke resulteerden in "composite" klinische manifestaties. Uit deze casussen blijkt dat heterogene klinische fenotypes niet altijd worden veroorzaakt door een enkel gendefect, met name in consanguine families, aangezien de aanwezigheid van homozygote regio's het risico verhoogd op het erven van meerdere recessieve genetische defecten. Daar waar kandidaat-gen benaderingen vaak te kort komen in het volledig verklaren van een klinische beeld, en er vaak meerdere rondes van klinische onderzoeken aan vooraf gaan, laten wij zien dat WES een volledige en accurate oplossing biedt om "blended" en "composite" manifestaties die voortkomen uit meerdere gendefecten volledig op te lossen. Bij het stellen van een genetische diagnose in heterogene aandoeningen gaan klinische bepalingen en WES data analyse soms hand in hand. In hoofdstuk 4 laten we zien dat klinische data essentieel kunnen zijn, niet alleen om een selectie van kandidaat WES-varianten te maken, of de klinische ernst van het genetisch defect te definiëren, maar ook om andere patiënten te identificeren met een defect in het zelfde gen. In een pediatrische patiënt met uitgesproken neurologische symptomen, resulteerde WES in het identificeren van een nieuwe homozygote CLPP mutatie. Op basis van het gendefect en de klinische symptomen werd de diagnose Perrault syndroom type 3 (PRLTS3) gesteld. Hersen MRI bij de patiënt liet specifieke afwijkingen zien, welke we hebben gebruikt om vergelijkbare patiënten te identificeren in de Amsterdam brain-MRI database, welke meer dan 3000 ongeclassificeerde gevallen van leukoencephalopathy omvat. In twee niet-gerelateerde patiënten met identieke MRI afwijkingen werden nieuwe CLPP missense mutaties geïdentificeerd. Onze data laat daarom zien dat gelijkenissen in 
hersen-MRI patroon gebruikt kunnen worden bij het identificeren van nieuwe PRLTS3 patiënten, met name in een vroeg stadium van ziekte ontwikkeling, wanneer maar een deel van symptomen aanwezig zijn.

Ons tweede doel was het karakteriseren van de functionele effecten van mutaties in bekende en nieuwe genen, daarbij gebruik makend van patiënt cellijnen en het zebravis model. In hoofdstuk 5 hebben we een complex I deficiënte patiënt met bewegingsintolerantie onderzocht, bij wie dieet-studies lieten zien dat het uithoudingsvermogen is spier toenam onder een vetrijk dieet vergeleken met een koolhydraatrijk dieet. We hebben WES uitgevoerd in deze patiënt om het genetische defect te karakteriseren en hebben een pathogene mutatie geïdentificeerd in het TMEM126B gen, welke een vroege assemblage factor van complex I codeert. Een complementatie studie in fibroblasten van de patiënt bevestigde dat het TMEM126B defect de oorzaak was van de complex I deficiëntie en dat deze voortkwam uit een onvolledige assemblage van de perifere arm van complex I. Op basis van een in vitro assay met patiënt fibroblasten, toonden we aan dat behandeling met het verzadigde vetzuur palmitaat resulteerde in een toename in maximale OXPHOS capaciteit, wat in lijn is met de klinische verbeteringen in de patiënt onder een vetrijk dieet. Het derde doel was om op basis van de betrokken genen en processen, nieuwe therapeutische targets te identificeren. Op basis van de veelbelovende resultaten in de TMEM126B patiënt, hebben we dezelfde assay toegepast op fibroblasten van patiënten met verschillende complex I gen defecten, en lieten zien dat ook andere vroege assemblage defecten in subunits en assemblage factoren van complex I (NDUFS7 en NDUFAF5) reageren op palmitaat behandeling, terwijl late assemblage defecten niet lijken te reageren. Onze data laat op een klinisch en biochemisch niveau zien dat een vetrijk dieet voordelig kan zijn voor bepaalde complex I patiënten, en dat onze cellijn assay gebruikt kan worden als een middel om patiënten die mogelijk baat hebben bij een dergelijk therapeutisch dieet te selecteren (hoofdstuk $\mathbf{5}$ ).

In hoofdstuk 6, hebben we WES uitgevoerd op een patiënt met Hirschprung disease (HSCR) en linkszijdige ptosis, waarbij we een nieuwe missense mutatie hebben geïdentificeerd in het $E R B B 2$ gen. Het patiënt fenotype toonde opvallende gelijkenis met een eerder gerapporteerde conditionele mutante erbB2 muis, in welke een gebrek aan functioneel erbB2 expressie tot een, vergelijkbaar met het humane, HSCR fenotype leidde, tevens gecombineerd met ptosis. De mutatie veroorzaakt een waarschijnlijk pathogene aminozuursubstitutie in het Juxta-membraan domein van de ERBB2 receptor, maar zou mogelijk ook een cryptische donor splice-site kunnen veroorzaken. Desondanks, bevestigden onze testen op RNA niveau dat er geen cryptische splicing plaatsvond, en tevens dat er geen sprake was non-sense mediated decay (NMD) in de patiënt fibroblasten. Daarnaast waren er op basis van in 
vitro ERBB2 overexpressie studies geen indicaties dat de ERBB2 activiteit was veranderd als gevold van de mutatie of dat de membraan lokalisatie was beïnvloed. Vandaar dat we op basis van deze in vitro studies niet onomstotelijk kunnen bewijzen dat de variant leidt tot inactivatie van ERBB2. Bewijs voor een rol voor ERBB2 in HSCR vereist daarom nadere functionele testen, bij voorkeur om het effect te onderzoeken van dit gen op het enterische zenuwstelsel. We zullen daarom het Phox- $2 b$ reporter-zebravismodel gaan gebruiken om het enterische zenuwstelsel te bestuderen na knock-out/knock-down van ERBB2 met behulp van CRISPR/Cas9. Alhoewel het zebravis model is gevalideerd als een bruikbaar model om nucleaire gendefecten te bestuderen, is er nauwelijks data bekend omtrent het mtDNA en de mechanismen betrokken bij segregatie van het mtDNA. Vandaar dat we in hoofdstuk 7 de mtDNA "bottleneck" in zebravissen bestuderen, om de grootte, timing, en variatie van deze bottleneck in geslachtscellen en somatische cellen op te helderen. Een volwassen zebravis oocyt bevat gemiddeld $\sim 19.0 \times 10^{6} \mathrm{mtDNA}$ moleculen met grote variatie onder de verschillende oocyten. Wij tonen aan dat tijdens de embryogenese het aantal mtDNA kopieën afneemt tot 170 mtDNA moleculen per primordial germ cell (PGC), een aantal dat vergelijkbaar is in zoogdieren, en afneemt tot $\sim 50$ moleculen per niet-PGC (somatische cellen). Deze bottleneck vindt plaats tijdens eenzelfde fase in de ontwikkeling, implicerende dat er een aanzienlijke variatie is in mtDNA kopieën in (niet-)PGCs van het zelfde vrouwtje, bepaalt door de variatie in de mature oocyt. Vandaar dat verschillen tussen de bottleneck in geslachtscellen en somatische cellen als gevolg van vroege PGC differentiatie, verantwoordelijk kunnen zijn voor de verschillende verdelingspatronen van familiale mtDNA mutaties in de mens.

In hoofdstuk $\mathbf{8}$ geven we een overzicht van de genetische modellen welke gebruikt worden om mitochondriële aandoeningen te bestuderen. Vooral met de komst van WES, is er vraag naar een genetisch model dat het testen van variant schadelijkheid in een relatief snelle en eenvoudige manier mogelijk maakt, in het bijzonder voor varianten met een onbekende significantie (VUS) en varianten in nieuwe genen. Geschikte uitleesmodellen om de schadelijkheid van varianten te testen kunnen worden gebaseerd op in silico analyse, in vitro studies of in vivo modellen. We geven een overzicht van de verschillende strategieën om de schadelijkheid van mutaties in mitochondriële aandoeningen functioneel te valideren, en bediscussiëren de voor- en nadelen van elk model. Echter, uiteindelijk zal de keuze voor een specifiek genetisch model volledig afhangen van de onderzoeksvraag, implicerende dat er geen opzichzelfstaand model is dat voldoet aan alle vraagstellingen. Ondanks de huidige successen in het identificeren van de genetische oorzaken van mitochondriële aandoeningen, is de vertaalslag naar effectieve behandelingsstrategieën nog beperkt, dit gezien de meeste behandelingsopties slechts ondersteunend zijn. Voor een gedeelte is dit vanwege de enorme genetische en fenotypische heterogeniteit binnen de mitochondriële 
aandoeningen. Ook bediscussiëren we de verschillende generieke behandelingsstrategieën welke momenteel worden gebruikt of getest, gebaseerd op fysieke training, diëten, en farmaceutische moleculen (stimulatie van biogenesis, ETC functie en anti-oxidant capaciteit), en welke als doel hebben het verbeteren van de mitochondriële capaciteit door een toename in mitochondriële biogenesis of het verbeteren van de ETC functie. In tegenstelling tot generieke behandelingsstrategieën richten de doelgerichte, gepersonaliseerde behandelingsstrategieën zich op het specifieke genetische of metabole defect in de patiënt. Gerichte behandeling kan omvatten het toedienen van specifiek enzymen, substraten of nucleotiden om zodoende de metabole deficiëntie te herstellen, ofwel, in de vorm van genetische manipulatie (verschuiven van mtDNA heteroplasmie, genetisch modificeren van nDNA mutaties, of "gene transfer" therapie) of stamcel therapie. Vanwege de grote genetische, biochemische en klinische heterogeniteit, is het onwaarschijnlijk dat een "one size fits all" benadering de oplossing zal zijn, maar dat juist de gerichte therapieën en specifieke combinaties van behandelen (mogelijk ook in combinatie met generieke therapieën) nodig zijn om effectief een specifieke subgroep van mitochondriële ziekte aan te pakken, uiteindelijk resulterende in een meer gepersonaliseerde behandelingsvorm.

\section{Conclusie}

We hebben aangetoond dat whole exome sequencing ons in staat stelt om de genetische oorzaak te identificeren in $69 \%$ van de patiënten met een mitochondriële aandoening, in wie geen oorzaak kon worden vastgesteld met behulp van conventionele, gerichte sequencing methoden. Nieuwe bioinformatica middelen, zoals WeGET, hebben bijgedragen aan het selecteren van veelbelovende kandidaat genen gebaseerd op co-expressie data. WES is daarom de geprefereerde, en meest geschikte benadering om het genetisch defect te karakteriseren in patiënten met een mitochondriële aandoening, met name in consanguine families, aangezien zij een verhoogd risico hebben op het overerven van meerdere genetische defecten. Op basis van complementatie studies in patiënt cellijnen, hebben we zowel bekende als nieuwe genetische defecten kunnen karakteriseren en de functionele consequenties van het defect kunnen bewijzen. Daarnaast, hebben we aangetoond dat de zebravis een geschikt model is om mitochondriële ziekte te bestuderen, niet alleen als model voor nucleaire defecten, zoals vaak gerapporteerd in literatuur, maar ook om mtDNA transmissie te bestuderen. In een beperkt aantal gevallen heeft het karakteriseren van de genen en processen welke betrokken zijn bij mitochondriële aandoeningen geleid tot het identificeren van nieuwe therapeutische targets. We hebben aangetoond dat behandeling op basis thiamine, riboflavine en een vetrijk dieet voordelen kan hebben voor patiënten met specifieke mitochondriële gendefecten (SLC19A3, SLC25A32, ACAD9, TMEM126B). Dit laat zien dat de behandeling van mitochondriële aandoeningen uiteindelijk in grote mate 
Samenvatting

gepersonaliseerd zal gaan worden, en zal vragen om nieuwe, additionele experimentele benaderingen. 
VALORISATION 
Valorisation 


\section{Relevance of this thesis in patient care}

The work presented in this thesis is not only relevant to improve our understanding of the genetic causes and pathophysiological mechanisms in mitochondrial disease from a research perspective, but it has also direct implications for the genetic diagnosis and in some cases opens novel options for personalized treatment of patients with mitochondrial disease in a clinical setting.

\section{WES in establishing a genetic diagnosis}

Until quite recently the genetic testing of a patient relied on the targeted sequencing of known disease genes based on the patient's phenotype, where Sanger sequencing was considered the "gold standard" in diagnostic testing (1). Such approach required many timeconsuming and expensive clinical and laboratory investigations in order to find a lead with previously reported gene defects, resulting in sequential sequencing of many candidate genes and a relatively low yield. As shown in this thesis, the development of next-generations sequencing (NGS) approaches, such as WES, has allowed the detection of genetic variants in all genes in parallel in the complete exome or genome of a patient. Current sequencingby-synthesis platforms, such as the Illumina NovaSeq 6000 are, depending on the coverage depth and read-length, capable of sequencing up to 200 human exomes or 48 genomes in a single run. Both the costs and time for sequencing a complete exome or genome has rapidly declined over the last couple of years, making it a cost-effective strategy to apply in clinical diagnostics (2). Therefore, WES has revolutionized the identification of potential disease causing variants in rare genetic diseases, which despite their individual scarcity, affect approximately $6-8 \%$ of the population during life-time (3).

In both our experience and that of others (4), WES is a particularly powerful tool to identify the genetic cause in heterogeneous disorders, in which most often no evident candidate genes are available. Where conventional sequencing methods were reported to solve no more than $11 \%$ in a heterogeneous patient cohort (5), the work in this thesis shows that WES is performing much better in heterogeneous, mitochondrial disorders. Using a 2-step NGS approach, we could identify a genetic defect in the mtDNA of $20 \%$, and an exonic defect in $\sim 50 \%$ of the patients, overall solving $\sim 70 \%$ of our patient cohort (chapter 2 ). It should be stressed, that this is a carefully selected cohort and the diagnostic yield in clinical practice will be lower. Interestingly, we showed that $\sim 30 \%$ of the gene defects in mitochondrial patients, covering both novel and known mitochondrial gene defects, were not included in the MitoCarta database, and therefore could missed when using targeted, gene panel based sequencing approaches. It is preferable to start directly with a complete exome and skip 
panel-based intermediate steps, as the choice for a panel is often arbitrary, never complete and genes and mutations are missed, causing unnecessary delay, when the complete exome needs to be analyzed as a second step. Based on our data, and that of others, we can conclude that WES is a comprehensive and effective method to find the genetic cause in mitochondrial disease, justifying the rapid introduction of WES in a diagnostic setting, and having direct implications for the establishment of a clinical genetic diagnosis in patients with mitochondrial disease.

In a clinical context, the complete exome should be analyzed completely, and one should not stop after the identification of a single candidate. This is not possible in gene panels, giving another argument for skipping this step in-between. As we show in chapter 3, patients with a multi-genic cause are not uncommon, especially in consanguineous families, and all exonic variants need to be evaluated for being disease causing, allowing the establishment of an accurate and complete genetic diagnosis in heterogeneous disorders that are caused by multiple gene defects. This is illustrated by the two patients with a broad variety of clinical symptoms, where the presence of multiple gene defects adds another level of complexity to the genetic diagnosis. In such cases, multiple gene defects (ACY1, ANTXR2, SERAC1) may appear as a single disease due to significantly overlapping (blended) phenotypic manifestations, or the individual gene defects (HPS1, BICD2) could present as non-overlapping (composite) disease phenotypes in the patient. We showed that WES is a powerful tool to resolve multi-genic disease, yet, one should ensure that analysis of the complete exome is being continued until each individual symptom can be genetically explained. It is important to understand the contribution of each individual gene defect to the disease phenotype, either in isolation or combination with the other defects, not only for prognosis and treatment, but also to avoid disease transmission. This especially applies to patients from consanguineous parents, who potential have a higher risk of inheriting multiple genetic defects than outbred families due to the presence of large homozygous genomic regions.

The improvements in identification of mtDNA and nuclear DNA defects, and the implementation of NGS based strategies in diagnostic setting allows is not only important for a rapid diagnosis. As illustrated by the patient with a genetic defect in the FAD transporter SLC25A32 (chapter 8), understanding the genetic basis of disease may directly result in a more effective treatment. In this case supportive treatment with riboflavin, a vitamin B2 precursor of FAD, will compensate for the lack of FAD, which functions as a crucial cofactor in the mitochondrial electron transport chain. A genetic diagnosis also allows the prevention of disease transmission by prenatal diagnosis (PND), as it gives parents the option to abort a pregnancy, or, if parents would opt for preimplantation genetic diagnosis (PGD), to select 
for mutation free embryos (6). WES is a relatively fast strategy, as illustrated by the quick establishment of a genetic diagnosis in a pediatric patient with encephalomyopathy, in who an FBXL4 gene defect was identified during another pregnancy of his mother, allowing timely prenatal testing of the unborn child (7). WES based preconception carrier screening (PCS) is a next step to estimate this risk of transmitting a recessive genetic disorder, even prior to the first pregnancy, and is especially valuable in couples who are at risk of transmitting multiple diseases, for example due to consanguinity (8). PCS is currently made available to all healthy couples, as the method seems sensitive and cost-effective enough for this.

Our data illustrates the power of WES as a tool to characterize the genetic defects underlying a patient's disease manifestations, but much more valuable information can be extracted from a patient's exome dataset. As WES detects all variations in the patient's exome, it will also include variants, which might lead to treatable disease or which define side-effects or efficacy of drugs. One might argue that one is obliged to provide the patient with all clinically-actionable or potentially clinically actionable information from the WES as this is not so much different from the heel prick test. Obviously, this is more complex for variants, causing late-onset disease, risk- factors or variants of unknown significance. This will require adequate counselling, informed consent and strict guidelines in terms of the genetic information that is reported back to the patient. But eventually, genome data will not be available for patients only, but will become standard information for every person in our society, entering the era of personalized, genome-based medicine.

WES will also and always result in the identification of novel, unknown, variants or variants of unknown significance in known or novel genes, complicating the interpretation of WES data. The development of novel bioinformatics tools to predict variant pathogenicity and gene function, for example based on gene conservation, 3D protein structure, or co-expression data (WeGET tool, chapter 8), is therefore crucial for interpreting WES variants, just as for the more recent WGS applications, which rely on the estimation of intronic- and splicevariants. As a result, validation and eventually implementation of novel bioinformatics tools, laboratory assays or genetic (animal) models to interpret WES variants, has direct impact on the diagnostic yield in a patient cohort. In chapter $\mathbf{5}$, we showed that lentiviral complementation in patient fibroblasts can be used for testing novel gene defects in OXPHOS deficient patients. Also in vivo models have a prominent role in establishing a relation between gene and phenotype or in understanding the transmission of mitochondrial disease (9). In chapter 7 we show that the zebrafish is a reliable model to study mtDNA mutations and their transmission. Still, implementation of such models in diagnostic setting will require standardization, validation and a sufficient throughput and speed to establish the genetic model in order to keep up with the fast generation of NGS data. 


\section{Compound testing in patient cell-lines}

As described in chapter $\mathbf{5}$, patient cell-line based assays can be a powerful tool to study the responsiveness of a patient to specific drugs or compounds in the patient's genetic context. The latter is crucial, as other mutations, but also polymorphisms, in the nuclear DNA and mtDNA can influence the pharmacological responsiveness and toxicity to a specific compound. As shown in chapter 5, despite the fact that TMEM126B and ACAD9 function in the same complex I assembly complex, the TMEM126B patient significantly responded to palmitic acid treatment by an increase in mitochondrial respiration, whereas the ACAD9 patient did not. The latter illustrates that individual patients with a similar disease, biochemical defect, or even similar gene defect or mutation, might respond differently to a specific treatment. Responsiveness to a certain compound should therefore be evaluated individually. Our patient-cell line assay, based on the Seahorse XF96, answers to this need for personalized medicine, as it allows the testing of different compounds, concentrations, and treatment durations in patient cells based on mitochondrial respiration parameters. Whereas in clinical practice, different diets or drugs are often subsequently tested in the patient, without knowing the effective components, our cell-line assay could overcome these limitations as a tool to identify patient responsiveness to specific FFAs, nutrients or drugs. Although, cultured fibroblasts are relatively easy to test in this assay, as patient skin biopsies can easily be taken and fibroblasts are often readily available in diagnostic centers, these cells might not always display a mitochondrial biochemical defect. Therefore, studying mitochondrial respiration parameters in muscle precursors or other cell types might be an interesting alternative. Although, our assay allows the identifying of compounds that improve mitochondrial respiration in the patient, it does not reveal clinical efficacy or toxicity, and eventually a complete pathway based analysis is needed to judge the potential for treatment and estimate possible toxic side-effects. In chapter 8, we report an example of possible pathway-based intervention in patient cells with an MTFMT defect, where targeting PDF (enzyme responsible for deformylation of methionine) with antibiotics and compound inhibitors should shift the substrate towards an increased formylated state and rescue mitochondrial translation. Assessment of this predicted rescue pathway should be performed at different molecular levels (e.g. formylation state, enzyme activity, mitochondrial translation) to judge the effectiveness of such compounds. Besides, negative or toxic effects of a compound, such as ROS production, lipid peroxidation, altered mitochondrial membrane potential and integrity, should be examined, preferably in the patient's own cells, as also toxicity levels dependent on the unique genetic background of the patient. This is illustrated by the observation that low levels of ROS might already become toxic in some patients with an OXPHOS defect, whereas others can cope with much higher ROS levels as they are able to upregulate their natural anti-oxidant defense system 
(10). For the future, it will therefore be important to assess all aspects of mitochondrial functioning and toxicity in these patient cell-lines, implementing parameters for all these mitochondrial processes in a single patient cell-line based assay to identify promising compounds or therapies for an individual patient. 


\section{References}

1. Bakker E. Is the DNA sequence the gold standard in genetic testing? Quality of molecular genetic tests assessed. Clinical chemistry. 2006;52(4):557-8.

2. Vissers L, van Nimwegen KJM, Schieving JH, Kamsteeg EJ, Kleefstra T, Yntema HG, et al. A clinical utility study of exome sequencing versus conventional genetic testing in pediatric neurology. Genet Med. 2017;19(9):1055-63.

3. Puiu M, Dan D. Rare diseases, from European resolutions and recommendations to actual measures and strategies. Maedica. 2010;5(2):128-31.

4. Pronicka E, Piekutowska-Abramczuk D, Ciara E, Trubicka J, Rokicki D, Karkucinska-Wieckowska $A$, et al. New perspective in diagnostics of mitochondrial disorders: two years' experience with whole-exome sequencing at a national paediatric centre. J Transl Med. 2016;14(1):174.

5. Neveling K, Feenstra I, Gilissen C, Hoefsloot LH, Kamsteeg EJ, Mensenkamp AR, et al. A posthoc comparison of the utility of sanger sequencing and exome sequencing for the diagnosis of heterogeneous diseases. Hum Mutat. 2013;34(12):1721-6.

6. Sallevelt SC, Dreesen JC, Drusedau M, Spierts S, Coonen E, van Tienen FH, et al. Preimplantation genetic diagnosis in mitochondrial DNA disorders: challenge and success. J Med Genet. 2013;50(2):125-32.

7. van Rij MC, Jansen FA, Hellebrekers DM, Onkenhout W, Smeets HJ, Hendrickx AT, et al. Polyhydramnios and cerebellar atrophy: a prenatal presentation of mitochondrial encephalomyopathy caused by mutations in the FBXL4 gene. Clinical case reports. 2016;4(4):4258.

8. Sallevelt S, de Koning B, Szklarczyk R, Paulussen ADC, de Die-Smulders CEM, Smeets HJM. A comprehensive strategy for exome-based preconception carrier screening. Genet Med. 2017;19(5):583-92.

9. Tyynismaa $\mathrm{H}$, Suomalainen A. Mouse models of mitochondrial DNA defects and their relevance for human disease. EMBO reports. 2009;10(2):137-43.

10. Voets AM, Lindsey PJ, Vanherle SJ, Timmer ED, Esseling JJ, Koopman WJ, et al. Patient-derived fibroblasts indicate oxidative stress status and may justify antioxidant therapy in OXPHOS disorders. Biochim Biophys Acta. 2012;1817(11):1971-8. 


\section{DANKWOORD}


Dankwoord 
Na vele wetenschappelijke woorden voorafgaand, wil ik nu graag de mensen bedanken die mij hebben geholpen tijdens mijn promotietraject, zowel in wetenschappelijke zin als op persoonlijk vlak. Het was na bijna vijf jaar studeren, waarbij vele onderwerpen de revue hebben gepasseerd, dat ik tijdens de stage bij Clinical Genomics mijn interesse voor de genetica ontdekte. Ik ben dan ook erg dankbaar dat Bert mij de kans heeft gegeven om een promotieonderzoek te starten binnen zijn afdeling, dit mede dankzij de subsidie van het Prinses Beatrix Spierfonds. Naast dat ik ontzettend veel geleerd heb in deze periode over de onderwerpen binnen mijn eigen proefschrift, heb ik dankzij de vele wetenschappelijke interacties en gemeenschappelijke meetings een goed beeld kunnen krijgen van de verscheidenheid aan onderzoeken, en de gang van zaken in de mitochondriële patiëntenzorg. Behalve dat het natuurlijk flink aanpoten is tijdens een promotie traject, heb ik het vooral ook als een prettige periode ervaren, met een gemoedelijke samenwerking met mijn promotor en collega's.

Graag wil ik mijn waardering uitspreken voor mijn promotor Bert, niet alleen voor zijn kennis en kunde, maar ook om zijn eigenschappen als mentor. Ik ben dankbaar voor zijn steun, adviezen en altijd kalme manier waarop hij kennis en vaardigheden overbrengt. Ik heb het als een fijne en leerzame samenwerking ervaren, waarbij ik niet alleen veel heb mogen leren over het uitvoeren van wetenschappelijk onderzoek, maar ook op het vlak van communiceren, schrijven, en zelfstandig werken als onderzoeker. Zijn manier van werken is altijd optimistisch en geruststellend, op moeilijke momenten in het onderzoek heeft mij dat geholpen om gemotiveerd te blijven. Bert is altijd wel in voor een goede sarcastische grap, ook tijdens de meetings. Alhoewel dat erg amusant is, was ik vaak zelf de pineut.

Ook wil ik mijn co-promotores René en Radek bedanken voor de prettige en leerzame samenwerking. Met veel plezier ben ik altijd naar het RoMa overleg in Rotterdam geweest, waar René met veel passie en kennis over zijn patiënten kon spreken.

Uiteindelijk hoop je de tijdens het promotie-traject opgedane kennis toe te mogen passen als zelfstandig onderzoeker. Ik mag dan ook van geluk spreken dat Peter en Michel mij de kans hebben gegeven om als onderzoeker binnen de afdeling Dermatologie aan de slag te gaan, en ik ben hen dankbaar voor het feit dat ze mij de ruimte hebben geven om dit proefschrift te voltooien. Ik ben inmiddels een jaar in dienst en werk met veel plezier en interesse aan de verschillende onderzoekslijnen. Ik ben dankbaar voor de fijne samenwerking met Peter, en tevens onze amusante gesprekken over de flamenco, en muziek in algemeen. Ook ben ik dankbaar voor de fijne samenwerking met Michel, binnen de genodermatosis, een richting die mooi aansluit bij mijn interesses in de genetica. Het onderzoek naar basaal cell carcinoma biedt voor mij een nieuwe uitdaging, waar ik samen met Klara en alle andere collegae met veel plezier aan werk, en waar hopelijk mooie resultaten uit gaan komen. Inmiddels hebben 
we al een aantal gezellige borrels achter de rug, onder andere in Lunteren, waar ik heb geleerd dat je bij het quizzen in Michel zijn team moet zitten om hoog te eindigen.

Ik wil mijn paranimfen Debby en Bianca bedanken voor de hulp en de gezellige momenten. Debby, ik heb veel van jou geleerd tijdens onze intensieve samenwerking de afgelopen jaren, en ben je daar zeer dankbaar voor! Mike, bedankt voor het delen van jou kennis en ervaring als onderzoeker, het heeft me zeer geholpen, zowel op het lab als bij het opschrijven van de data. Dat laatste geldt ook voor Rick, de drijvende kracht op het lab, altijd behulpzaam en bereid om op de cruciale momenten van een experiment te helpen of advies te geven. Ook Will en Marike zijn heel behulpzaam geweest bij het uitvoeren van een aantal belangrijke metingen, dank hiervoor. Uiteraard geen WES data zonder de heren van de bioinformatica, Bart, Fons, jullie zijn onmisbaar geweest in deze studies en ik heb veel respect voor jullie kennis.

Ik wil mijn oude AlO-kamer bedanken voor de fijne en gezellige jaren met vooral veel humor. Ivo, Auke, met jullie heb ik veel kunnen lachen en fijn kunnen samenwerken. Ivo, ik hoop nog vaak met je te mogen gaan hardlopen, zwemmen of mountainbiken, ondanks dat je me vaak volledig hebt afgemat. Auke wens ik heel veel succes toe als post-doc in de verenigde staten. Ik wil verder alle collega's van Clinical Genomics bedanken voor de vriendelijke en gezellige werkomgeving de afgelopen jaren, de gezellige borrels, en dagjes-uit.

Jazz-musicus Emiel van Egdom wil ik bedanken voor het overdragen van zijn kunde op de gitaar, dit blijft toch mijn grote passie. Ik heb de afgelopen jaren veel van je mogen leren als musicus. Immers, "If you lack technique you lose the freedom to create" (Paco de Lucia). Ook wil ik Ber, mijn amigo op de spaanse gitaar, bedanken voor de vele gezellige en gepassioneerde muzieksessies.

Ruby, wij doen praktisch altijd alles gezellig samen, en tot voorkort zelfs nog synchroon promoveren. Dat was heel prettig, al was het af en toe toch ook goed dat er nog een computerscherm tussen ons in stond. Samen vervelen we ons nooit, met al onze gemeenschappelijke interesses, de muziek, schilderkunst en het mountainbiken, krijgen we de dagen altijd goed gevuld. Bedankt voor al je hulp bij het promoveren, maar ook voor het lekker avondeten dat altijd klaarstaat en het smeren van de boterhammen. Arnou, Maria, Fleur en Stefan, bedankt voor de gezellige momenten samen en de ontspannende avonden tussen al het promoveerwerk door. Prisca, gelukkig heb ik zo een slimme zus als jij, waarvan ik altijd heel veel profijt heb gehad. Na je promotie aan het Erasmus woon je nu weer fijn in het zuiden bij ons, en heb je het goed naar je zin bij de Pathologie. Toon, Niek, en oma, bedankt voor jullie steun en liefde, en dat ik altijd met Ruby kan binnenvallen voor gezelligheid. 


\section{APPENDIX}

Curriculum Vitae

List of publications 
Appendix 


\section{Curriculum Vitae}

\section{Personal details:}

Name:

Address:

Date of birth:

Place of birth:

Nationality:

Gender:

E-mail:

Tel:

\section{Education:}

(January 2017-present)

(July 2012- present)
Tom Elisabeth Joseph Theunissen

Oude Heirbaan 96 bus 1

3620 Lanaken

Belgium

31-05-1989

Bilzen (Belgium)

Dutch

Male

tom.theunissen@mumc.nl

+31649671586

\section{Postdoctoral researcher:}

Dept. of Dermatology, Maastricht University Medical Centre + (MUMC+), Maastricht, The Netherlands. Laboratory of Prof. dr. Peter M. Steijlen. Research topics: Genodermatosis, Basal cell carcinoma (BCC).

\section{PhD candidate:}

Thesis title: "Novel causes, mechanisms and therapeutic strategies in mitochondrial disease". Dept. of Clinical Genomics, School of GROW, Maastricht University, Maastricht, The Netherlands. Promotor: Prof. dr. Bert Smeets (Dept. of Clinical Genomics, Maastricht University), co-promotors: dr. Irenaeus de Coo (Dept. of Neurology, Erasmus MC), dr. Radek Szklarczyk (Dept. of Clinical Genetics, MUMC+). This project was financed by Princess Beatrix Spierfonds, grant application: W.OR11-24

(2010-2012)

\section{Master of Science:}

Molecular life sciences: Oncology and Developmental Biology, GROW institute, Maastricht University

(2007-2010)

\section{Bachelor of Science}

Molecular life sciences, transnational University Limburg (tUL), Maastricht University 
(2001-2007)

Internships:

(MSc senior)

(MSc Junior)

(BSc Thesis)

\section{Secondary School}

College: Porta Mosana College Maastricht

Profile: VWO, N\&G

Dept. of Clinical Genomics, Maastricht University, Alma-in-Silico partnership with Liege University (ULg). Thesis title: "Pathological processes involved in mitochondrial dysfunction due to mtDNA replication defects. Supervisor: Prof. dr. Bert Smeets

Dept. of Toxicology, Maastricht University, NUTRIM institute. Project title: "The influence of Hif- $1 \alpha$ overexpression on $B(\alpha) P$ induced nucleotide excision repair in renal cell-lines". Supervisors: Prof. dr. Roland Chiu, dr. Marten Schults

Dept. of Biochemistry, subdivision Structural Bioinformatics, Maastricht University, CARIM institute. Thesis title: "Thrombin as a potential target for small inhibitory molecules". Supervisors: Prof. dr. Gerry Nicolaes, dr Mahesh Kulharia

\section{Additional work experience:}

Student Assistant at dept. of Biochemistry, division Bioinformatics, Maastricht University (01-07-2010/ 05-09-2010). Continuation of the in silico "Thrombin inhibitor design" project. Project: Performing optimizations on 3D protein structure modeling and virtual ligand screening using protein docking programs such as FRED, Autodock, ICM. Supervisor: Prof. dr. Gerry Nicolaes

\section{Awards:}

- $\quad$ GROW science day award 2014 (pitch, poster presentation)

\section{Certificates and courses:}

- $\quad$ Art.9 Officer (Animal course)

- Safe Microbiological Techniques (SMT) License

- Grant writing course

- Scientific English writing course (Language Center)

- Scientific presentation course 


\section{Teaching:}

- Supervision of Biomedical Bachelor student, Senior internship (2017, Bachelor thesis)

- Supervision of Biomedical Master student, Senior internship (2013, master thesis and defense)

- Supervision of Biomedical Bachelor student, Senior internship (2016, Bachelor thesis)

\section{Other interests:}

Classical guitar, educated by musician Emiel van Egdom (Berklee college of music method), 2010-present. 
Appendix 


\section{List of publications}

Theunissen TEJ, Nguyen M, Kamps R, Hendrickx ATM, Sallevelt SCEH, Gottschalk RWH, Calis CM, Stassen APM, de Koning B, Mulder-Den Hartog ENM, Schoonderwoerd K, Fuchs SA, Hilhorst-Hofstee Y, de Visser M, Vanoevelen J, Szklarczyk R, Gerards M, de Coo IFM*, Hellebrekers DMEI*, Smeets HJM*. Whole exome sequencing is the preferred, first strategy to identify known and novel causes of mitochondrial disease. Submitted

Theunissen TEJ*, Sallevelt SCEH*, Hellebrekers DMEI, de Koning B, Hendrickx ATM, van den Bosch BJC, Kamps R, Schoonderwoerd K, Szklarczyk R, Mulder-Den Hartog ENM, de Coo IFM, Smeets HJM. Rapid Resolution of Blended or Composite Multigenic Disease in Infants by Whole-Exome Sequencing. The Journal of pediatrics. 2017;182:371-4.e2. doi: 10.1016/j. jpeds.2016.12.032

Theunissen TEJ, Szklarczyk R, Gerards M, Hellebrekers DMEI, Mulder-Den Hartog ENM, Vanoevelen J, Kamps R, de Koning B, Rutledge SL, Schmitt-Mechelke T, van Berkel CG, van der Knaap MS*, de Coo IFM*, Smeets HJM*. Specific MRI Abnormalities Reveal Severe Perrault Syndrome due to CLPP Defects. Frontiers in neurology. 2016;7:203. doi: 10.3389/ $\underline{\text { fneur.2016.00203 }}$

Theunissen TEJ, Gerards M, Hellebrekers DMEI, van Tienen FH, Kamps R, Sallevelt SCEH, Hartog E, Scholte HR, Verdijk RM, Schoonderwoerd K, de Coo IFM*, Szklarczyk R*, Smeets HJM*. Selection and Characterization of Palmitic Acid Responsive Patients with an OXPHOS Complex I Defect. Frontiers in molecular neuroscience. 2017;10:336. doi: 10.3389/ $\underline{\text { fnmol.2017.00336 }}$

Theunissen TEJ*, Hellebrekers DMEI*, de Koning B, Gerards M, Szklarczyk R, Mulder-Den Hartog ENM, de Coo IFM, Smeets HJM. An ERBB2 mutation as a potential, novel cause of Hirschprung's disease. Manuscript in preparation

Otten ABC, Theunissen TEJ, Derhaag JG, Lambrichs EH, Boesten IB, Winandy M, van Montfoort AP, Tarbashevich K, Raz E, Gerards M, Vanoevelen J, van den Bosch BJC, Muller $\mathrm{M}$, Smeets HJM. Differences in Strength and Timing of the mtDNA Bottleneck between Zebrafish Germline and Non-germline Cells. Cell reports. 2016;16(3):622-30. doi: 10.1016/i. celrep.2016.06.023 
Hellebrekers DMEI, Sallevelt SCEH, Theunissen TEJ, Hendrickx ATM, Gottschalk RWH, Hoeijmakers JGJ, Habets DD, Bierau J, Schoonderwoerd K, Smeets HJM. Novel SLC25A32 mutation in a patient with a severe neuromuscular phenotype. European journal of human genetics : EJHG. 2017;25(7):886-8. doi: 10.1038/ejhg.2017.62

Kamps R, Szklarczyk R, Theunissen TEJ, Hellebrekers DMEI, Sallevelt SCEH, Boesten IB, de Koning B, van den Bosch BJC, Salomons G, Mendes M, Verdijk RM, Schoonderwoerd K, de Coo IFM, Vanoevelen J, Smeets HJM. Genetic defects in mtDNA-encoded protein translation cause pediatric, mitochondrial cardiomyopathy with early-onset brain disease. European journal of human genetics: EJHG. 2017. doi: 10.1038/s41431-017-0058-2. [Epub ahead of print] 